\title{
WestVirginiaUniversity
}

THE RESEARCH REPOSITORY @ WVU

Graduate Theses, Dissertations, and Problem Reports

2010

\section{Correlating simulated surface marks with near-surface tornado structure}

\author{
Michael I. Zimmerman \\ West Virginia University
}

Follow this and additional works at: https://researchrepository.wvu.edu/etd

\section{Recommended Citation}

Zimmerman, Michael I., "Correlating simulated surface marks with near-surface tornado structure" (2010). Graduate Theses, Dissertations, and Problem Reports. 3206.

https://researchrepository.wvu.edu/etd/3206

This Dissertation is protected by copyright and/or related rights. It has been brought to you by the The Research Repository @ WVU with permission from the rights-holder(s). You are free to use this Dissertation in any way that is permitted by the copyright and related rights legislation that applies to your use. For other uses you must obtain permission from the rights-holder(s) directly, unless additional rights are indicated by a Creative Commons license in the record and/ or on the work itself. This Dissertation has been accepted for inclusion in WVU Graduate Theses, Dissertations, and Problem Reports collection by an authorized administrator of The Research Repository @ WVU.

For more information, please contact researchrepository@mail.wvu.edu. 


\title{
CORRElATING SIMULATED SURFACE MARKS WITH NEAR-SURFACE TORNADO STRUCTURE
}

\author{
Michael I. Zimmerman
}

Dissertation submitted to the Eberly College of Arts and Sciences at West Virginia University

In partial fulfillment of the requirements for the degree of

\author{
Doctor of Philosophy \\ in \\ Physics
}

\author{
Mark E. Koepke, Ph.D., Chair \\ David C. Lewellen, Ph.D \\ Earl E. Scime, Ph.D. \\ H. Arthur Weldon, Ph.D. \\ Kenneth Showalter, Ph.D.
}

Department of Physics

Morgantown, WV

2010

Keywords: tornado structure, tornado dynamics, corner flow structure, corner flow dynamics, debris, surface marks

(C)2010 Michael I. Zimmerman 


\begin{abstract}
Correlating simulated surface marks with near-surface tornado structure
\end{abstract}

Michael I. Zimmerman

Tornadoes often leave behind patterns of debris deposition, or "surface marks", which provide a direct signature of their near surface winds. The intent of this thesis is to investigate what can be learned about near-surface tornado structure and intensity through the properties of surface marks generated by simulated, debris-laden tornadoes. Earlier work showed through numerical simulations that the tornado's structure and intensity is highly sensitive to properties of the near-surface flow and can change rapidly in time for some conditions. The strongest winds often occur within tens of meters of the surface where the threat to human life and property is highest, and factors such as massive debris loadings and asymmetry of the main vortex have proven to be critical complications in some regimes. However, studying this portion of the flow in the field is problematic; while Doppler radar provides the best tornado wind field measurements, it cannot probe below about $20 \mathrm{~m}$, and interpretation of Doppler data requires assumptions about tornado symmetry, steadiness in time, and correlation between scatterer and air velocities that are more uncertain near the surface.

As early as 1967, Fujita proposed estimating tornado wind speeds from analysis of aerial photography and ground documentation of surface marks. A handful of studies followed but were limited by difficulties in interpreting physical origins of the marks, and little scientific attention has been paid to them since. Here, Fujita's original idea is revisited in the context of three-dimensional, large-eddy simulations of tornadoes with fully-coupled debris.

In this thesis, the origins of the most prominent simulated marks are determined and compared with historical interpretations of real marks. The earlier hypothesis that cycloidal surface marks were directly correlated with the paths of individual vortices (either the main vortex or its secondary vortices, when present) is unsupported by the simulation results. Cycloids in the simulations arise from debris deposited beneath the central annular updraft that has converged from a much larger area and are modulated by turbulent fluctuations in debris amount. Other classes of marks noted in the literature such as "lineation" and "scalloping" are also reinterpreted. Variations in the shapes, sizes, and spacings of surface marks with the most critical dimensionless parameters characterizing near-surface and debris cloud structure are explored. Analysis techniques are presented to capture the geometric properties of marks in some regimes, and possibilities for inferring near-surface vortex flow scales from mark properties are discussed. The prospects are promising enough to warrant documentation of surface marks when available (likely through aerial photography), particularly for cases where useful Doppler measurements have been gathered. 


\section{Acknowledgements}

I would like to offer sincere, heartfelt thanks to my co-advisors, Dr. David Lewellen and Dr. Mark Koepke, for giving me the opportunity to achieve one of my dreams: to complete my doctoral degree in Physics. I have been incredibly grateful for the chance to tap into their deep knowledge of physics, to work under their patient and enthusiastic scientific guidance, and to witness their flexibility and optimism when faced with complex issues. Throughout my research, they have provided advice, insight, and friendship that has been invaluable to me. I am thankful for their assistance with my thesis, and I owe my committee a debt of gratitude for taking the time to review it.

I also owe thanks to Dr. Earl Scime and Dr. Jack Littleton, who provided my very first research opportunity at WVU. Dr. Scime's guidance as Department Chair and physics instructor has been very much appreciated. I am grateful for having had the chance to complete coursework under the Physics Department faculty who tirelessly helped me to lay the basic foundations for a career in physics. I thank Dr. Holger Kersten and Dr. Ralf Basner for facilitating my off-campus field experience in Greifswald, Germany, and Dr. Dimitris Vassiliadis, Dr. Paul Cassak, and Dr. Herbert Gunell for useful discussions on physics research and career options. Thank you to Siobhan Byrne, Sherry Puskar, Sandy Johns, and Devon Cleland for their assistance throughout the years. I learned a great deal under the watchful patience of Phil Tucker, Carl Weber, Doug Mathess, and Tom Milam. To Dr. Vladimir Demidov, thank you for providing summer financial support for me.

Saikat Thakur, Maggie Wei, Jon Blessington, Sean Finnegan, Paul Miller, Jeff Walker, Mattias Tornquist, Stephanie Sears, Josh Miller, Holly Henderson, Jered Studinsky, Alison Mynsberg, Sean Kremins, and Allison and Andy Keller, I am glad to have spent time studying and hanging out with you.

Finally, I would like to thank my wife, Jen, who has provided loving support during my graduate student career, through late nights of research, weeks of studying for qualifiers, and intense days of thesis preparations. To Kelvin, thanks for keeping me grounded; evenings 
spent playing blocks, trains, and hide-and-seek with you have been some of the best moments of my life. I look forward to showing Kelvin and baby Noah the world. To my mom, Patty, thanks for watching Kelvin at a moment's notice, for always reminding me that I could achieve anything I could imagine, and for helping to support my family when times got tough. To my grandparents, Jim and Frances, and to Uncle Jimmy, you have provided consistent models of honesty, hard work, and determination, and I am extremely grateful for your support throughout the years. To Megan and Mark, thank you for always being there to talk, and thank you for your generosity in helping us when we needed it. To my mother-in-law, Lee, thank you for always being supportive of us.

This material is based upon work supported by the National Science Foundation under Grants No. 0635681 and AGS-1013154. Any opinions, findings, and conclusions or recommendations expressed in this material are those of the authors and do not necessarily reflect the views of the National Science Foundation. 


\section{Contents}

1 Introduction $\quad 1$

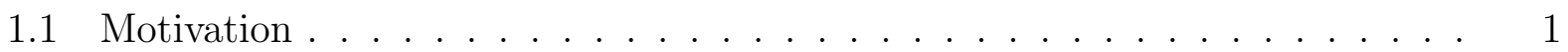

1.2 Corner flow and debris dynamics . . . . . . . . . . . . . . . . . . . 4

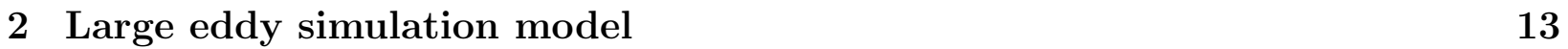

2.1 Air dynamics . . . . . . . . . . . . . . . . . . . . 13

2.2 Debris dynamics . . . . . . . . . . . . . . . . . . . . . 15

2.3 Simulation procedures and default parameters . . . . . . . . . . . . 18

3 Simulation of surface tracks $\quad 21$

3.1 Surface track accounting and measures . . . . . . . . . . . . . . . . 21

3.2 General origins of surface marks . . . . . . . . . . . . . . . . . 31

3.3 Turbulent variability of surface tracks . . . . . . . . . . . . . . 33

3.4 Sensitivities to simulation parameters . . . . . . . . . . . . 33

3.4.1 Sensitivities to surface parameterization . . . . . . . . . . 33

3.4 .2 Grid resolution . . . . . . . . . . . . . . . . 42

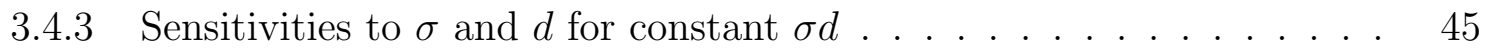

3.5 Multiple debris species . . . . . . . . . . . . . . . . . . 49

4 Simulation parameters varied $\quad 55$

4.1 Parameters involved . . . . . . . . . . . . . . . . 55 
4.2 Dimensional analysis . . . . . . . . . . . . . . . . 56

4.3 Bounding values on physical and dimensionless parameters . . . . . . . 58

5 Taxonomy and detailed physical origins of marks 61

5.1 Deposition underneath the central vortex . . . . . . . . . . . . . . 62

5.2 Deposition by debris-laden surface rolls . . . . . . . . . . . . . . . . 73

5.3 Deposition of fully lofted debris $\ldots \ldots$. . . . . . . . . . 80

5.4 Effects of debris availability $\ldots \ldots \ldots \ldots$

5.5 Time-varying cases $\ldots \ldots \ldots \ldots \ldots$. . . . . . . . . . . . . . 87

$\begin{array}{lll}6 & \text { Quantitative analyses } & 93\end{array}$

6.1 Downstream averages to infer near-surface vortex radius and shift $\ldots . . .93$

6.2 Orientation histogram to capture surface mark shapes . . . . . . . . . . . . 95

6.2.1 Extracting the average "fingerprint" mark . . . . . . . . . . . . . . . 99

6.2.2 Extracting a near-surface swirl velocity scale . . . . . . . . . . 100

6.3 Analyses to infer surface mark spacings . . . . . . . . . . . . . . . . . . 104

$\begin{array}{lll}7 & \text { Conclusions and discussion } & 113\end{array}$

$\begin{array}{lr}\text { A Tables of runs } & 119\end{array}$ 


\section{List of Tables}

3.1 Statistics on debris loading and net deposition under different values of the surface parameter $c_{a}$ (from the runs represented in Figure 3.13). . . . . . .

3.2 Statistics on debris loading and net deposition under different values of the surface parameter $c_{b} \ldots \ldots \ldots \ldots$. . . . . . . . . . . . . . . 42

A.1 A subset of stationary runs without debris used as initial conditions for translating, debris-laden cases. . . . . . . . . . . . . . . . . 120

A.2 A subset of low-swirl runs regridded from run3320-tav. . . . . . . . . . . . 120

A.3 A subset of high-swirl runs regridded from run3240-tav. . . . . . . . . . . . . 120

A.4 A subset of medium-swirl runs regridded from run3300-tav. . . . . . . . . . . 121

A.5 A subset of high-swirl runs regridded from run2057-tav. . . . . . . . . . . . 122

A.6 A subset of multispecies, medium-swirl runs regridded from run3300-tav. . . 123 


\section{List of Figures}

1.1 Comparison of corner flow structure in axisymmetric, stationary high- and medium-swirl cases. . . . . . . . . . . . . . . . . . . 5

1.2 A photograph of a real tornado with a debris cloud (Whipple, 1982). . . . 6

1.3 A cutaway snapshot of simulated debris cloud development, showing qualitative simularity to the debris cloud in 1.2, from Gong (2006). . . . . . . . . . 7

1.4 Aerial imagery of real surface tracks, showing some of the variety of marks observed in the field. From Fujita (1981). . . . . . . . . . . . . . . . 7

1.5 Satellite imagery of Martian dust devil tracks, from the HiRISE experiment (Image: NASA/JPL/University of Arizona). . . . . . . . . . . . . . . 8

1.6 Two measures of near-surface intensification versus corner flow swirl ratio, showing peak intensification at about $S_{C}=1$.4. From Lewellen et al. (2000).

3.1 Representative surface tracks of net debris deposition and instantaneous debris flux at the surface for three sample high-swirl, translating tornadoes. . . . . 23

3.2 As in Figure 3.1 except for a sample of medium-swirl tornadoes. . . . . . . . 24

3.3 Surface tracks of peak flow quantities for the case of Figure 3.1e,f. . . . . . . 25

3.4 As in Figure 3.3 except for the the medium-swirl case in Figure 3.2e,f (run

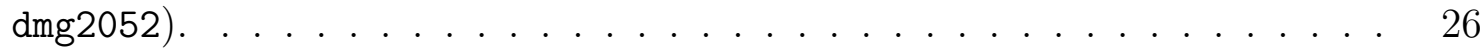

3.5 Surface tracks of time-integrated flow quantities for the cases of Figures 3.1e,f and $3.2 \mathrm{e}, \mathrm{f} . \ldots \ldots \ldots \ldots \ldots \ldots$ 
3.6 Directional wind markers taken at the first grid point above the surface for a collection of high-swirl tornadoes. . . . . . . . . . . . . . . . . 28

3.7 As in Figure 3.6, except for a collection of medium-swirl tornadoes. . . . . . $\quad 29$

3.8 Simulated time-dependent pressure traces taken by stationary probes near the surface. . . . . . . . . . . . . . . . . . .

3.9 Comparison of the surface track from a medium-swirl case with surfacerelative translation and the surface track collected artificially from an otherwise identical stationary case, demonstrating the importance of the full surface interaction in modulating local debris fluxes at the surface. . . . . . . . . . 32

3.10 High- and medium-swirl tracks of net deposition demonstrating differences in turbulent variability of the most prominent marks. . . . . . . . . . .

3.11 Instantaneous snapshots of vertical surface debris flux at different instants along the track in Figure 3.10a. . . . . . . . . . . . . . . . . . 35

3.12 As in figure 3.11, except for the track in Figure 3.10b. . . . . . . . . . . . 35

3.13 Deposition tracks from medium- and high-swirl cases with different removal

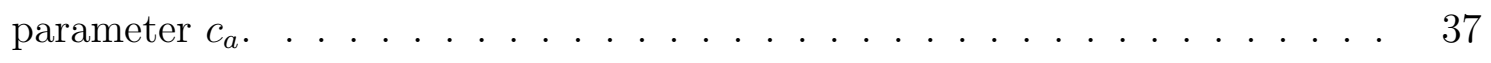

3.14 Sample instantaneous surface debris fluxes for the tracks in Figure 3.13. . . . 38

3.15 Sample surface tracks for medium- and high-swirl tornadoes with different surface parameters $c_{d}$ and $c_{t} \ldots \ldots \ldots \ldots \ldots$

3.16 Sample instantaneous surface debris fluxes for the tracks in Figure 3.15. . . . 41

3.17 Sample surface tracks for medium- and high-swirl tornadoes with different surface parameter $c_{b} \ldots \ldots \ldots \ldots \ldots$

3.18 Sample instantaneous surface debris fluxes for the tracks in Figure 3.17. . . . 44

3.19 Net deposition patterns from simulated medium-swirl tornadoes with different central resolution. . . . . . . . . . . . . . . . . . . . . 46

3.20 Net deposition patterns from simulated high-swirl tornadoes with different central resolution. . . . . . . . . . . . . . . . . . 
3.21 Comparison of high-swirl deposition tracks in Figure 3.20, panels d (run dmg2028) and f (run dmg2014e) with a Gaussian-filtered version of panel f, showing that despite acquiring finer-scale features, the coarse features of the higher-resolution track (panel f) qualitatively resemble those at lower resolution (panel d) after the fine-scale features are filtered out. . . . . . . . . . . . 48

3.22 Comparison of medium- and high-swirl deposition tracks with different $\sigma$ and $d$ while holding the product $\sigma d$ constant. . . . . . . . . . . . . . . . . . 50

3.23 Deposition tracks for multispecies runs with different subgrid turbulence debris pickup parameter $c_{a} \ldots \ldots \ldots \ldots$. . . . . . . . . . . . 52

3.24 Deposition tracks from a medium-swirl collection of single-species runs with varying $c_{a}$ for comparison with the multispecies tracks in Figure 3.23. . . . .

5.1 Fujita et al.'s taxonomy of marks emphasizing secondary vortices as the primary physical process behind deposition marks, from Fujita (1976). . . . . .

5.2 Fujita et al.'s model of a single vortex collecting debris into a spot at the ground. Some debris is able to follow the corner flow and is ejected aloft. From Fujita $(1976) \ldots \ldots \ldots \ldots \ldots$

5.3 Deposition tracks for a collection of tornadoes with $A_{a} \sim 2$ and $A_{v} \sim 9$, primarily varying $S_{c}$ and $A_{t} \ldots \ldots \ldots \ldots$. . . . . . . . . 64

5.4 Sample instantaneous surface debris fluxes for the cases in Figure 5.3. . . . . 65

5.5 Deposition tracks for a collection of tornadoes with $A_{a} \sim 8$ and $A_{v} \sim 11$, primarily varying $S_{c}$ and $A_{t} \ldots \ldots \ldots 6$

5.6 Sample instantaneous surface debris fluxes for the cases in Figure 5.5. . . . . 67

5.7 Deposition tracks for a collection of tornadoes with $S_{c}=3.0$ and $A_{a}=7.7$, primarily varying $A_{v}$ and $A_{t} \ldots \ldots \ldots$. . . . . . . . . . . 68

5.8 Sample instantaneous surface debris fluxes for the cases in Figure 5.7. . . . . 69

5.9 Deposition tracks for a collection of tornadoes with $S_{c}=12.4$ and $A_{a}=1.9$, primarily varying $A_{v}$ and $A_{t} \ldots \ldots \ldots \ldots$. . . . . . . . . . 70 
5.10 Sample instantaneous surface debris fluxes for the cases in Figure 5.9. . . . . 71

5.11 Azimuthally averaged vertical debris flux and angular momentum from a highswirl, stationary tornado, showing up/down debris flux couplet responsible for generating cycloidal marks (in translating cases). . . . . . . . . . . . 74

5.12 Sample cycloidal marks with instantaneous surface debris fluxes. . . . . . . 75

5.13 Aerial imagery of a tornado damage track, showing a qualitative change in cycloidal marks from left to right across the image. From Fujita (1967a) . . . 76

5.14 Mean deposition level (nondimensionalized by air density $\rho$ ) versus $S_{c} A_{t}^{-1}$. $\quad 76$

5.15 Illustration of strong cycloidal marks being laid down in regions of convergence along the local streamlines. . . . . . . . . . . . . . . . . . . . 77

5.16 Instantaneous and time-averaged surface debris fluxes or a collection of highswirl tornadoes. . . . . . . . . . . . . . . . . . . . . 78

5.17 Deposition tracks showing variation with $A_{a}$ for sample medium- and highswirl collections of tornadoes. . . . . . . . . . . . . . . . . . . . . 79

5.18 Aerial imagery of a tornado damage track from Fujita et al. (1976b), showing

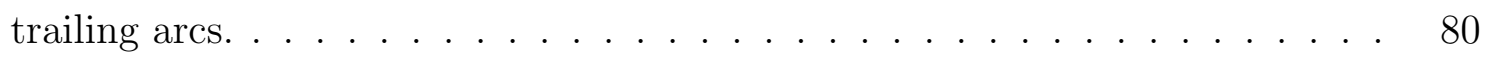

5.19 Sample trailing arcs with instantaneous surface debris fluxes. . . . . . . . . 81

5.20 Horizontal slices of instantaneous vertical air velocity at $\mathrm{z}=1.0 \mathrm{~m}$, showing existence of near-surface inflow rolls in the absence of debris. . . . . . . . . . 82

5.21 Convergence/divergence pattern observed in tall wheat stubble by DaviesJones et al (Davies-Jones et al., 1978). . . . . . . . . . . . . . .

5.22 Instantaneous snapshot of near-surface horizontal and vertical airflow and surface debris flux, showing correlation between deposition and localized regions of horizontal convergence. . . . . . . . . . . . . . . . . . . 84

5.23 Sample diffuse far-field swaths with instantaneous surface debris fluxes. . . . 85

5.24 Aerial imagery of a tornado damage track from Agee (1977), showing possible diffuse far-field swaths perhaps mischaracterized as drift marks (D). . . . . . 
5.25 Comparison of deposition tracks for medium- and high-swirl tornadoes moving over limited and unlimited surface debris fields. . . . . . . . . . . . . 87

5.26 Downstream averages of net deposition per length from a a collection of highswirl tornadoes with $A_{t}=0.36$ and different $A_{v}$, showing the progression from cycloidal deposition to scalloping. . . . . . . . . . . . . . . . . 88

5.27 Surface tracks from a high-swirl tornado moving over a field of "heavy" debris, demonstrating the extreme low- $A_{v}$ limit. . . . . . . . . . . . . . 89

5.28 Surface tracks from four simulated corner flow collapse tornado evolutions, from (Lewellen and Zimmerman, 2008). . . . . . . . . . . . . . . 91

5.29 Sample instantaneous surface debris fluxes from the respective corner flow collapse cases in Figure 5.28. . . . . . . . . . . . . . . . . . . .

6.1 Downstream averages from a collection of high-swirl tornadoes moving at different speeds, showing locations of dual deposition peaks. . . . . . . . . . 95

6.2 Time-averaged vertical surface debris flux from respective cases in Figure 6.1, showing near-surface flow features responsible for dual peaks of deposition. . 96

6.3 Vortex shift $\delta_{s}$ relative to $R_{C}$ aloft versus $A_{t} \ldots \ldots \ldots$. . . . . . . 97

6.4 Vortex shift $\delta_{s}$ relative to $R_{C}$ aloft versus $A_{t} / S_{C} \ldots \ldots \ldots$

6.5 Demonstration of the "orientation histogram" technique for extracting the mean geometric structure of surface marks in a track. . . . . . . . . . . . . 100

6.6 Demonstration of correlation between the second derivative of net deposition and local sharpness of surface marks. . . . . . . . . . . . . . . 101

6.7 (a) Ideal cycloid with $\mathrm{U} / \mathrm{V}=1$. (b) Resulting "chromosome" pattern for the

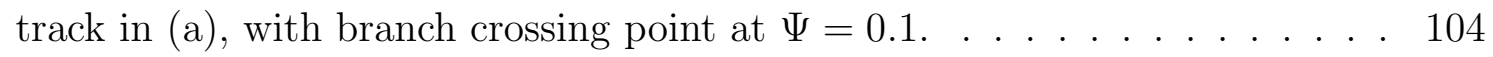

6.8 "Chromosome" branch crossing points from ideal cycloids versus U/V. The crossing point $\Psi_{\text {cross }}$ is equal to $U / V \ldots \ldots \ldots$. . . . . . . . . 105

6.9 Correlation of $A_{t}$ with "chromosome" crossing point. . . . . . . . . . . . 106 
6.10 Attempted correlation of the maximum horizontal windspeed at $\mathrm{z}=0.5 \mathrm{~m}$ and an effective near-surface swirl velocity inferred from the "chromosome" crossing point for a subset of simulation cases containing cycloids. . . . . . . . . . 107

6.11 Surface mark spacings inferred by hand-tracing cycloidal marks in a collection of high-swirl tornadoes with $S_{c}=12.4, A_{v}=12.2, A_{a}=2.39$, and varying $A_{t} . .109$

6.12 Autocorrelation along the tornado direction of travel for two selected deposition tracks. . . . . . . . . . . . . . . . . . . . . . 110

6.13 (a) Ideal cycloid with $\mathrm{U} / \mathrm{V}=0.5$. (b) Autocorrelation of the cycloidal signal in (a) along its direction of travel. . . . . . . . . . . . . . . . 111 


\section{Chapter 1}

\section{Introduction}

\subsection{Motivation}

The tornado vortex is one of the most visually-striking and destructive terrestrial fluiddynamic structures known. As evidenced by amateur and scientific video records tornadoes come in wide variety and can include visual signatures such as massive debris clouds and secondary vortices rotating around the main vortex. A tornado is typically embedded within a swirling parent thunderstorm which can be tens of kilometers wide and tall, consisting of a rapidly-swirling updraft with a radial scale of tens to hundreds of meters fed by a converging, swirling inflow along the surface. The upturn of the surface inflow to meet the swirling updraft aloft, which can be quite sharp, forms the "corner flow", which is the focus of this work. Visual representations of a subset of different corner flows are shown in Figure 1.1a,b, and corner flows with debris clouds are shown in Figures 1.2 and 1.3.

Lewellen and Lewellen (2007a,b); Lewellen et al. (2000) showed through simulations that corner flow structure and intensity are highly sensitive and sometimes rapidly responsive to properties of the near-surface inflow. They demonstrated that the highest winds are often produced in the corner flow just tens of meters above the surface where the threat to human life and property is highest. However, the near-surface portion of the flow is the most 
difficult to measure in the field: Doppler radar, which provides the best tornado wind field measurements, is unable to probe below about $20 \mathrm{~m}$, and interpretation of Doppler data can be complicated by uncertainties in vortex symmetry, steadiness, and differences between debris and air flow (e.g Bluestein and Unruh (1989); Dowell et al. (2005); Wurman et al. (1997)).

The destruction of man-made structures has been heavily studied and can provide critical information about near-surface tornado strength; however, coverage of ground structures is sparse and their interaction with the windfield generally complex (Doswell et al., 2009). In-situ probes are generally difficult to deploy and provide only a one-dimensional slice of data through the near-surface flow, although recent attempts have involved arrays of groundbased probes in concert with Doppler radar measurements aloft (e.g. Wurman et al. (2010)). Holland et al. (2006), Beck (2008), and Beck and Dotzek (2010) developed and applied analysis techniques allowing reconstruction of near-surface tornado winds from patterns of tree breakage based on previous work by Letzmann (1923a,b); Bech et al. (2009) also applied this methodology to a real case.

A direct, complementary signature of near-surface tornado winds often available when other markers are not is the pattern of debris removal and deposition at the surface. Such "surface marks" are left behind when a tornado traverses a roughly uniform patch of loose debris, such as a freshly-plowed field, some rows of dry corn stubble (e.g. Figure 1.4), or the dusty surface of Mars (e.g. Figure 1.5). The first extensive attempts to infer properties of near-surface tornado winds from surface marks were carried out by Fujita in the mid-1960s (Fujita, 1966) and were followed by a handful of other attempts.

The most critical limitation in earlier studies of surface marks were in uncertainties about the physical origins of marks. Based on aerial photographs of surface tracks, close inspection of marks on the ground, and observation of actual tornadoes, Fujita and colleagues developed a surface mark taxonomy (Fujita, 1966; Fujita et al., 1967, 1976a,b). This framework for understanding surface marks was centered around the interpretation that marks were primar- 
ily created through the action of individual vortices (either the main vortex or secondary vortices) collecting debris into distinct piles as they moved along. Fujita and colleagues developed a simple mathematical model to infer mean tangential velocities of secondary vortices rotating about the main vortex from geometric properties of cycloidal marks (Fujita, 1967; Fujita et al., 1970, 1976b); the estimated windspeeds agreed well with conventional photogrammetric observations of secondary vortex paths. Other researchers also collected aerial photography of surface tracks (Prosser, 1964), adpoted Fujita and colleagues' taxonomy (Davies-Jones et al., 1978), and employed Fujita and colleagues' analysis techniques to infer rotation velocities of secondaries about the main vortex (Agee et al., 1975, 1977; Fujita, 1981).

Though surface marks often resemble cycloids and secondary vortices have been observed to follow roughly cycloidal paths, secondary vortices in the field have not been directly correlated with any particular types of marks. The actual flow features responsible for surface marks have remained unknown primarily because high-resolution, low-level, timeresolved flow measurements have not been available and are still largely out of reach today (c.f. Bluestein and Golden (1993); Wurman et al. (1997)).

In the present work Fujita's general idea of inferring tornado properties from surface marks is resurrected through use of state-of-the-art large-eddy simulations (LES) of tornado corner flows with debris. The simulation code utilized was developed previously as part of a longstanding effort to study tornado structure and dynamics (e.g. Lewellen and Lewellen (2007a,b); Lewellen et al. (2000, 2008); Lewellen (1973, 1976); Lewellen et al. (1997); Xia et al. (2003)). It will be shown in the present work that there is no direct correlation between simulated secondary vortices and the most prominent signatures of deposition or removal. Rather, a convolution of removal and deposition by a handful of physical processes over space and time is responsible for forming distinct debris patterns at the surface. The importance of each process varies with a set of dimensionless governing parameters describing corner flow and debris cloud structure, and the resulting set of marks reflects the tornado's governing 
parameters.

The remainder of this chapter provides background information on corner flow and debris cloud structure and dynamics. A summary of the LES code and simulation procedures are provided in Chapter 2. The general physical origins of the most prominent simulated surface marks and tests to determine the sensitivity of surface mark properties to simulation parameters are described in Chapter 3. Chapter 4 justifies selection of the simulation set of over 150 runs based on the most important simulation and physical parameters affecting tornado and debris cloud structure. In Chapter 5, a taxonomy of simulated surface marks is developed, and surface marks' physical origins are interpreted in terms of critical dimensionless parameters of the system. Analysis techniques for extracting properties of marks are presented in Chapter 6, and conclusions and discussion of future prospects for inferring near-surface winds, tornado structure, and debris cloud structure from surface marks are provided in Chapter 7.

\subsection{Corner flow and debris dynamics}

Angular momentum is exactly conserved in a tornado flow away from the surface, and the typical state is one of approximate cyclostrophic balance in which the radial pressure gradient force balances inertia (c.f. Lewellen (1976)). Due to surface friction the swirl velocity (i.e. the azimuthal component of velocity) must go to zero at the surface, breaking cyclostrophic balance in the near-surface flow. However, the radial pressure gradient is inherited from aloft, driving a converging radial inflow that turns upward upon reaching the central vortex, forming the tornado corner flow. As the inflow separates from the surface it overshoots the radius at which its lowered angular momentum $\Gamma$ is equal to that in the core flow aloft . They demonstrated that the inertial overshoot can lead to quite dramatic velocity intensification in the corner flow near the surface: though angular momentum is typically depleted to some degree in the near-surface inflow, it is conserved once the flow separates from the ground, 

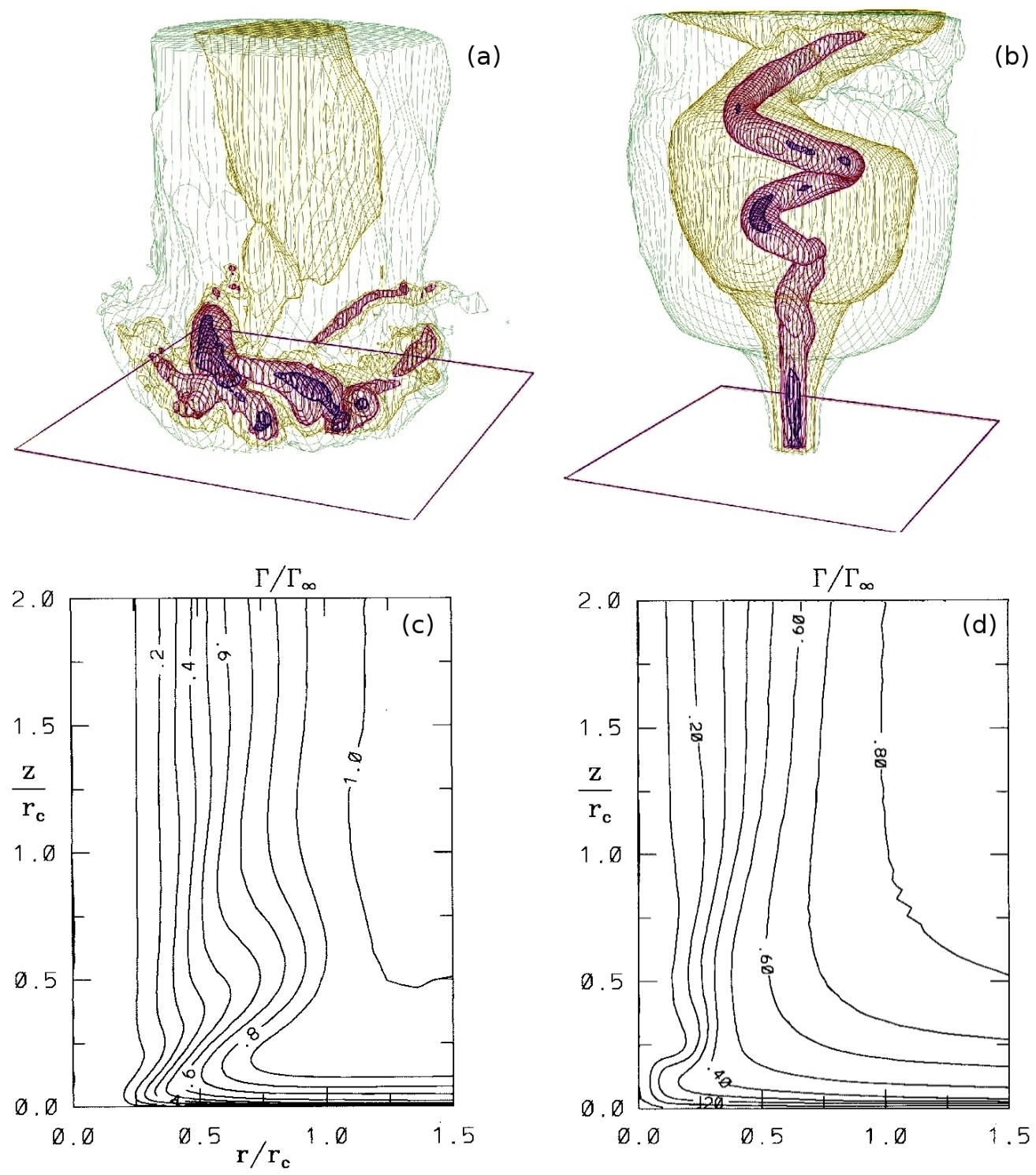

Figure 1.1: (a) Wireframe isosurfaces of instantaneous hydrodynamic pressure for a highswirl, stationary tornado corner flow. (c) Contours of axisymmetrically- and time-averaged angular momentum in the radial-vertical plane for a low-swirl corner flow. Angular momentum levels have been normalized to the far-field angular momemtum aloft, $\Gamma_{\infty}$, and length scales have been normalized to the vortex radius aloft, $R_{C}$. (b,d) As in $(\mathrm{a}, \mathrm{c})$ except for a low-swirl tornado. (a,b) are taken from Lewellen (2007); (c,d) are taken from Lewellen et al. (2000). 


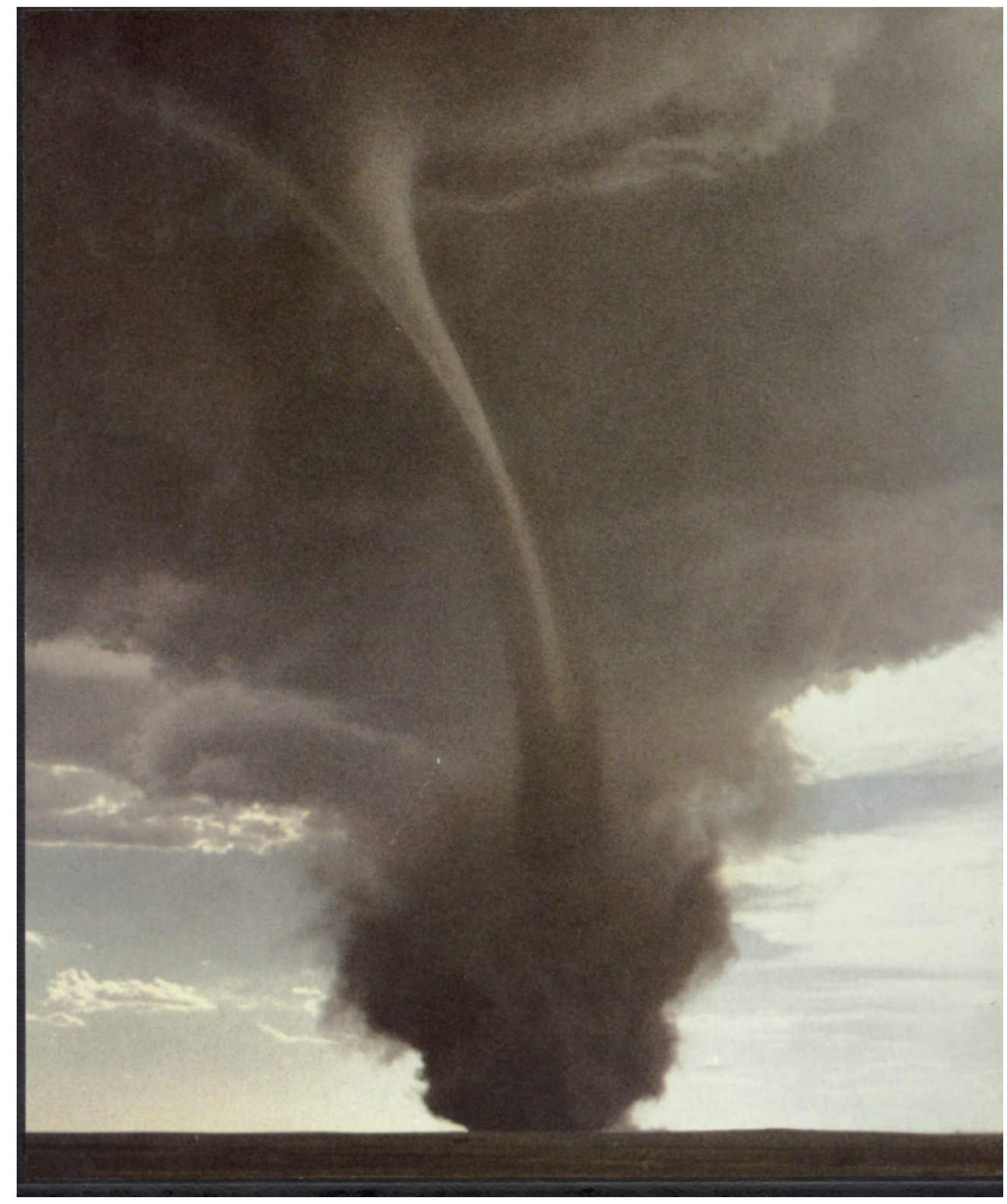

Figure 1.2: A photograph of a real tornado with a debris cloud (Whipple, 1982). 


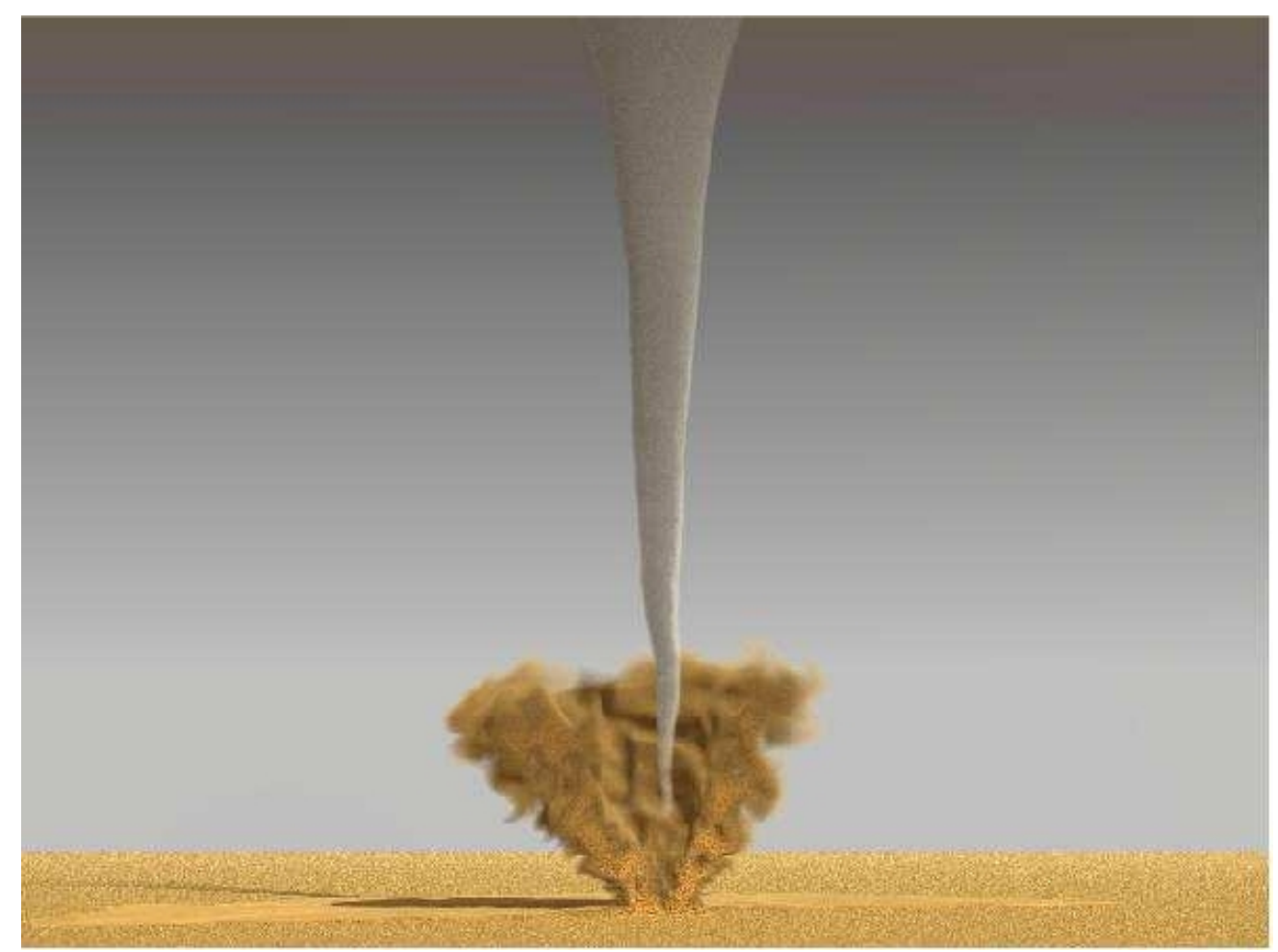

Figure 1.3: A cutaway snapshot of simulated debris cloud development, showing qualitative simularity to the debris cloud in 1.2, from Gong (2006).
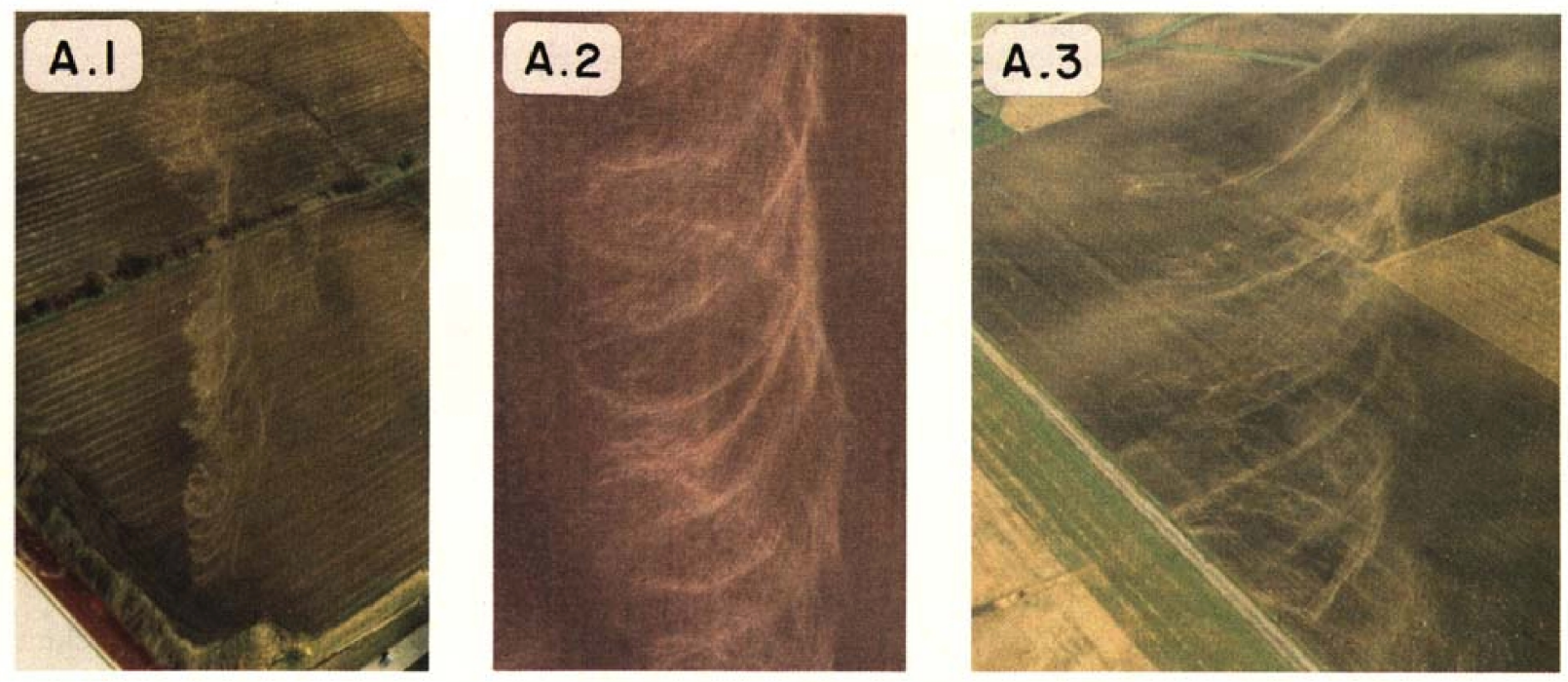

Figure 1.4: Aerial imagery of real surface tracks, showing some of the variety of marks observed in the field. From Fujita (1981). 


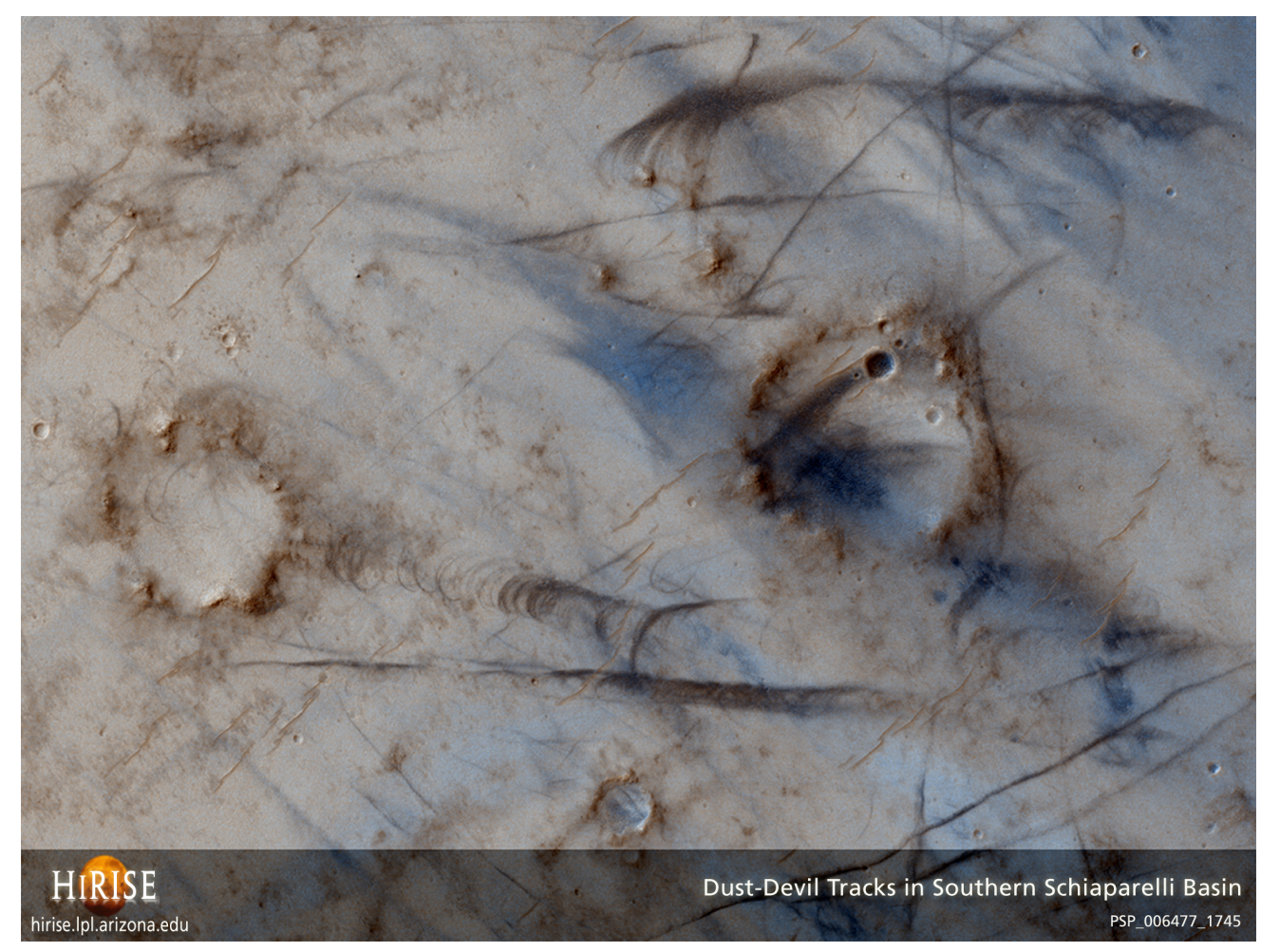

Figure 1.5: Satellite imagery of Martian dust devil tracks, from the HiRISE experiment (Image: NASA/JPL/University of Arizona).

causing the swirling flow to "spin up" as it contracts radially.

Lewellen et al. (2000) identified a critical corner flow swirl ratio capturing the effects of many physical parameters on tornado structure and velocity intensification. They demonstrated that (a) vortex structure and near-surface velocity intensification are highly sensitive to properties of the near-surface inflow and (b) corner flow structure can change rapidly in time under some conditions. Their corner flow swirl ratio is defined as

$$
S_{c}=\frac{R_{c} \Gamma_{\infty}^{2}}{\Upsilon}
$$

where $\Gamma_{\infty}$ is the angular momentum far from the central vortex aloft, $R_{c}$ is the characteristic radius of the core flow aloft (calculated in terms of the maximum mean swirl velocity in the vortex aloft, $\left.R_{c} \equiv \Gamma_{\infty} / V_{c}\right)$, and $\Upsilon$ is the flux of depleted angular momentum, $\Gamma_{d}=\Gamma_{\infty}-\Gamma$, through the vortex. $\Gamma_{d}$ is typically large only in a shallow layer just above the surface and 
within the central vortex core.

$S_{c}$ may be thought of as the ratio between characteristic swirl and radial velocity components in the surface inflow. In the "low-swirl" limit, the peak near-surface swirl velocity is pushed close to the center of the vortex, and vertical pressure gradients form a strong central updraft that experiences a vortex breakdown aloft (e.g. Figure 1.1b,d). In the "high-swirl" limit the corner flow upturn and corresponding inertial overshoot occurs further from $r=0$, reducing the potential amount of velocity intensification (relative to the low-swirl regime) and producing a roughly annular updraft that sometimes surrounds a central downdraft (e.g. Figure 1.1a,c). Secondary vortices are common in the radial angular momentum gradients of high-swirl corner flows, though their presence is not directly parameterized by $S_{c}$ (Lewellen et al., 2000).

In Lewellen and Lewellen (2007a); Lewellen et al. (2000), the mean near-surface velocity intensification relative to $V_{c}$ aloft was shown to correlate with $S_{c}$, with a sharp intensification peak at $S_{C} \sim 1.4$ (e.g. Figure 1.6). Tornadoes with $1.4<S_{c}<2$ are here considered to be low-swirl, those with $2<S_{c}<4$ are considered to be medium-swirl, and those with $S_{c}>4$ are considered to be high-swirl. These separations are somewhat arbitrary but useful for categorization purposes.

Also, surface-relative translation $U_{\text {trans }}$ was shown by (Lewellen et al., 2000) to have a non-trivial effect on the distribution of angular momentum in the near-surface flow. Surface friction exerts non-axisymmetric torques on the swirling, translating inflow, tending to increase $\Gamma$ on the right side of the tornado and to decrease $\Gamma$ on the left side. The lowerangular momentum fluid is preferentially drawn into the main vortex, causing the vortex to shift and tilt in an effort to maintain local axisymmetry. Also, $\Upsilon$ is raised by the increase in low angular momentum fluid entering the central vortex, effectively reducing $S_{c}$.

Lewellen et al. (2004, 2008) and Gong (2006) added fine debris to the LES code and demonstrated that accumulation of debris in the surface layer can result in a massive debris cloud aloft, providing one of the most distinctive visual signatures of a tornado (e.g. Figures 
1.2 and 1.3). They showed that even though the debris cloud occupies only a small portion of the entire flow, vortex structure within hundreds of meters of the surface can be significantly affected by the accumulation of fine debris. Debris centrifuging, negative buoyancy, variable density, and differences between air and debris velocities were all shown to play a role.

Peak mean flow velocities were typically reduced by massive simulated debris loadings; however, debris tended to concentrate swirl momentum in the near-surface flow, increasing the damage potential of the vortex. Also, a negative feedback between debris loadings and air velocities was identified that limited the total amount of debris accumulated by the flow. In the low-swirl limit, centrifuging of debris tended to lower the radial component of inflow, pushing it toward an effectively higher swirl ratio with peak mean swirl velocities occuring at a larger radius. In the present work, quoted values for $S_{c}$ are those computed before debris was introduced, as are the radial and velocity scales of the vortex aloft, respectively $R_{c}$ and $V_{c}$.

Finally, Gong (2006) and Lewellen et al. (2008) introduced a set of dimensionless parameters capturing the leading effects of fine debris on tornado flows (these parameters are described in detail in Chapter 4 and used extensively in the present work). 


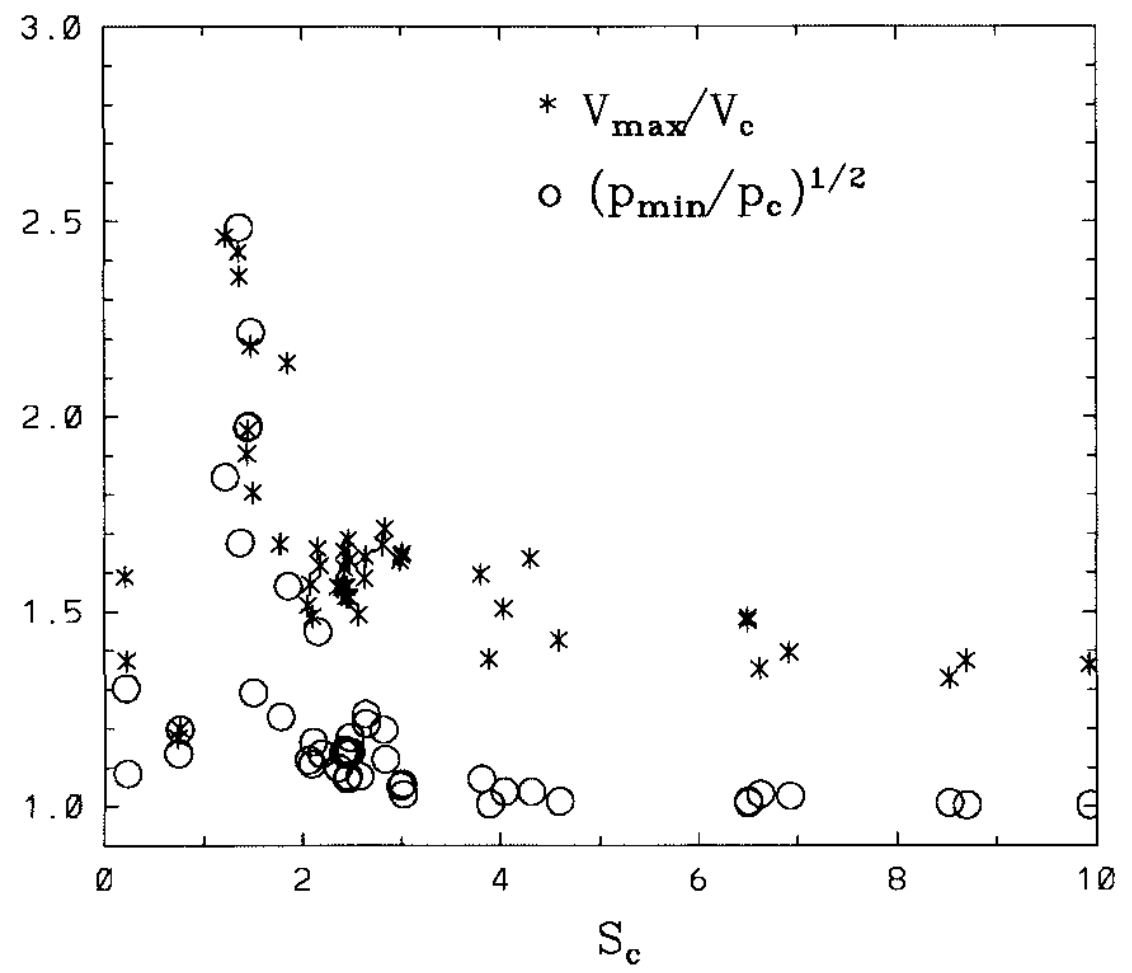

Figure 1.6: Two measures of near-surface intensification versus corner flow swirl ratio, showing peak intensification at about $S_{C}=1$.4. From Lewellen et al. (2000). 


\section{Chapter 2}

\section{Large eddy simulation model}

The large eddy simulation (LES) model used in this work was previously developed as part of a longstanding effort to understand tornado dynamics and structure (e.g. Lewellen and Lewellen (2007a,b); Lewellen et al. (2000, 2008, 1997)). Here, a brief summary of the simulation code as well as typical initial and boundary conditions are presented, and the default run parameters and procedures are discussed.

\subsection{Air dynamics}

It is currently unfeasible to perform direct numerical simulations of tornadoes due to the large range of length scales involved. Approximately $10^{19}$ grid points would be required to resolve a $2 \mathrm{~km}$ wide and tall cube containing a tornado at a resolution commensurate with the viscous length scale $\left(\sim 1 \mathrm{~mm}^{3}\right)$. Large eddy simulations (LES) circumvent this restriction by solving the Navier-Stokes equations explicitly only for those eddies most important to turbulent transport and modeling the energy transport into smaller, unresolved eddies. For tornadoes the relevant length scale of the most important eddies is on the order of tens of meters (Lewellen et al., 1997). The major drawbacks of this approach are that it requires a choice of subgrid turbulence parameterization and the grid points must be spaced closely enough to resolve the smallest important eddies. 
Technical details of the LES code utilized are given in Lewellen et al. $(2008,1997)$ and summarized here.

The continuity and momentum equations for unsteady, incompressible air flows are solved on a three-dimensional grid independently stretched along each dimension, using secondorder spatial differencing and a leapfrog scheme in time. With velocity components $u_{i}$, uniform density $\rho$, and pressure $p$, the equations are:

$$
\begin{gathered}
\frac{\partial u_{i}}{\partial x_{i}}=0 \\
\frac{\partial\left(\rho u_{i}\right)}{\partial t}+\frac{\partial}{\partial x_{j}}\left(\rho u_{i} u_{j}\right)=\frac{\partial}{\partial x_{j}}\left(-p \delta_{i j}+\tau_{i j}\right)+F_{d i}
\end{gathered}
$$

Summation over repeated indices is implied. Note the presence of the air-debris drag force $F_{d i}$ in the momentum equation, which is discussed in the next section.

Subgrid dynamics are implemented through the subgrid stress tensor, $\tau_{i j}$, which takes the form

$$
\tau_{i j}=-\nu\left(\frac{d u_{i}}{d x_{j}}+\frac{d u_{j}}{d x_{i}}\right)-\delta_{i j} q^{2} / 3
$$

where $\nu$ is the turbulent eddy viscosity, defined below, and $q^{2}$ is twice the subgrid turbulent kinetic energy. The quantity $q^{2}$ evolves according to

$$
\begin{gathered}
\frac{d q^{2}}{d t}+\frac{d\left(u_{i} q^{2}\right)}{d x_{i}}=-2 \tau_{i j} \frac{d u_{i}}{d x_{j}}+\frac{d}{d x_{i}}\left(K d q^{2} / d x_{i}\right)-\frac{q^{3}}{4 \Lambda} \\
K=q \Lambda / 3, \nu=q \Lambda / 4 \\
\Lambda=\min \left(c_{1} \max (\Delta x, \Delta y, \Delta z), .65 z, .5 q / N, .5 q / \xi\right)
\end{gathered}
$$

where $\Lambda$ is the subgrid turbulence length scale (representing the sizes of subgrid eddies) and is limited by the local grid spacing (through $\Delta x, \Delta y, \Delta z$ ), the distance to the surface (through $z$ ), and the characteristic displacement of a fluid parcel in a stably gravitationally stratified background fluid or a rotational gradient (through $q / N$ or $q / \xi$, where $N$ and $\xi$ are 
parcel oscillation frequencies in the respective environments).

Based on the methodology laid out in Lewellen et al. (1997) and later references, boundary flow conditions are imposed on the sides, top, and bottom of a $2 \mathrm{~km}$-wide and tall cube, reflecting conditions that might occur on kilometer scales within a thunderstorm (although no attempt is made to simulate any particular storm that has occurred in the field). More specifically, angular momentum and radial inflow profiles are specified versus height on the lateral boundaries, and except within a user-specified disk within which outflow conditions may be specfied, the upper boundary flow is initially adjusted to ensure global mass conservation. A zero-slope condition is imposed on tangential components of outflow on the lateral and top boundaries, and a no-slip condition is imposed at the surface with a surface roughness length, $z_{0}$. Typical boundary flow conditions used in this work are outlined in Section 2.3.

\subsection{Debris dynamics}

The assumption is made that the volume fraction occupied by debris is much less than one; this ensures that debris has no effective internal pressure due to debris-debris collisions. The two-fluid "dusty gas" momentum and continuity equations used to model a single debris species, with velocity components $u_{d i}$ and variable mass density $\rho_{d}$, are as in Lewellen et al. (2008); Marble (1970):

$$
\begin{gathered}
\frac{\partial \rho_{d}}{\partial t}+\frac{\partial\left(\rho_{d} u_{d i}\right)}{\partial x_{i}}=0 \\
\frac{\partial\left(\rho_{d} u_{d i}\right)}{\partial t}+\frac{\partial}{\partial x_{j}}\left(\rho_{d} u_{d i} u_{d j}\right)=\frac{\partial \tau_{i j}^{d}}{\partial x_{j}}-F_{d i}-\rho_{d} g \delta_{i 3}
\end{gathered}
$$

where the debris subgrid stress tensor, $\tau_{i j}^{d}$, is treated analogously to air, using the same subgrid turbulent kinetic energy and length scale. Note the presence of gravitational forcing on the debris (e.g. the third term on the righthand side of Equation 2.8). 
The air-debris drag force is given by

$$
\begin{gathered}
F_{d i}=\rho_{d} \frac{u_{d i}-u_{i}}{\tau_{v}} \\
\tau_{v}=\frac{4 d^{2} \sigma}{3 \mu C_{D} R e_{p}} \\
C_{D} \approx \frac{24}{R e_{p}}+\frac{6}{1+\sqrt{R e_{p}}}+0.4 \\
R e_{p} \equiv \rho d\left|\vec{u}_{d}-\vec{u}\right| / \mu
\end{gathered}
$$

where $\sigma$ and $d$ are the debris particle mass density and diameter, respectively, $\mu$ is the air viscosity, $C_{D}$ is an empirically derived drag coefficient for a spherical particle valid for $R e_{p}<2 \times 10^{5}$ (White, 1991), and $R e_{p}$ is the particle Reynolds number. In the simulation set $R e_{p}$ is generally well below the upper range of validity for $R e_{p}$; for example, using typical simulation parameters of $\rho=1 \mathrm{~kg} \mathrm{~m}^{-3}, d \sim 1 \mathrm{~mm}, \mu=1.78 \times 10^{-5} \mathrm{~kg} \mathrm{~m}^{-1} \mathrm{~s}^{-1}$, and $\left|\vec{u}_{d}-\vec{u}\right| \sim w_{t} \sim 10 \mathrm{~m} \mathrm{~s}^{-1}$ (where $w_{t}$ is the debris terminal velocity in freefall) produces $R e_{p} \sim 500$.

The LES code enables multiple debris species to be simulated simultaneously. There are no coupling terms between individual debris species since each is considered to be "pressureless", though they do couple indirectly through their respective interactions with air. Various limits are imposed on debris fields to avoid instabilities uniquely associated with simulating a pressureless fluid under the influence of a drag force, as outlined in the Appendix of (Lewellen et al., 2008).

The remaining implementation involves the surface fluxes of debris mass and momentum. The motion of a particles in a surface layer is governed largely by Shields' parameter

$$
\tau^{*}=\sqrt{\frac{\rho u_{*}^{2}}{\sigma d g}}
$$

where $u_{*}$ is the friction velocity. $\tau^{*}$ is essentially a nondimensionalization of the shear stress 
at the surface and can be thought of as the ratio of the aerodynamic force exerted on a particle to the particle's weight. Lewellen et al. (2008) pointed out that the two regimes of most relevance to debris-laden tornadoes are saltation (bouncing of particles along the surface) and lofting; the former occurs for $0.01<\tau^{*}<1$ while the latter occurs for $\tau>1$. In direct analogy in the LES code, debris may become entrained in the near-surface flow through two mechanisms: lofting by the subgrid turbulent airflow or lofting through collisions with debris that has already been lofted. Including the flux of debris returning to the surface, the total vertical debris flux at the surface is modeled in three pieces:

$$
\begin{gathered}
\mathcal{W}_{0}^{a}=c_{a} q_{1} \quad \text { for } \quad \frac{\rho \mathrm{q}_{1}^{2}}{\sigma \mathrm{dg}}>c_{\mathrm{t}} \quad \text { (lofting by subgrid turbulence) } \\
\mathcal{W}_{0}^{d}=c_{d} D_{1} u_{d}^{*} \quad \text { for } \quad \frac{\rho \mathrm{u}_{\mathrm{d}}^{* 2}}{\sigma \mathrm{dg}}>c_{\mathrm{t}} \quad \text { (lofting through debris impacts) } \\
\mathcal{W}_{0}^{-}=c_{b} \mathcal{W}_{1} \quad \text { for } \quad \mathcal{W}_{1}<0 \quad \text { (downward surface debris flux) }
\end{gathered}
$$

where $u_{d}^{*}$ is the debris friction velocity, $c_{a}, c_{d}$, and $c_{b}$ are simulation parameters controlling the relative importance of each term, $c_{t}$ is a threshold parameter for lofting, and default choices of the coefficients are $c_{a}=0.12, c_{d}=0, c_{b}=1, c_{t}=0.5$. Subscripts of 1 refer to quantities taken at the first grid point above $z=0$. The total debris flux to the surface is given by the sum of the three contributions in Equations 2.14-2.16:

$$
\mathcal{W}_{0}=\mathcal{W}_{0}^{a}+\mathcal{W}_{0}^{d}+\mathcal{W}_{0}^{-}
$$

The vertical flux of vertical debris momentum to the surface is modeled using three terms as well:

$$
\bar{w}_{d} \mathcal{W}_{0}=\mathcal{W}_{0}^{a} q_{1}+\mathcal{W}_{0}^{d} u_{d}^{*}+\left(2-c_{b}\right) w_{d 1} \mathcal{W}_{0}^{-} / c_{b}
$$

The first two terms represent momentum fluxes analogous to equations 2.14 and 2.15 , and the third term represents the momentum flux carried by debris impinging upon the surface. 
Setting $c_{b}=0$ causes all debris to bounce elastically (the corresponding momentum flux is then twice the momentum flux carried toward the surface by the particle before impact), and setting $c_{b}=1$ corresponds to all particles sticking upon impact.

Finally, the vertical flux of horizontal debris momentum to the surface is:

$$
\bar{w}_{d} \mathcal{U}_{0}=-\mathcal{U}_{1} \frac{u_{d}^{* 2}}{\left|\vec{u}_{d 1}\right|}+c_{u} \mathcal{U}_{1} \mathcal{W}_{0}^{-} / D_{1}
$$

and likewise for $\mathcal{V}_{1}$, where $\mathcal{U}_{1}$ and $\mathcal{V}_{1}$ are horizontal debris momentum components at the first grid point above the surface, and $c_{u}$ is a simulation parameter controlling the relative importance of contributions from debris (typically, $c_{u}=1$ ). The first term models frictional losses to the surface and the second term represents the vertical flux of horizontal momentum carried by falling debris particles. Setting $c_{u}=0$ ensures that debris loses no horizontal momentum to the surface upon impact.

One of the chief limitations of this model is in the fluid approximation used for debris. All debris particles in a grid cell are assumed to have the same trajectory; this assumption fails when debris particles entering a grid cell have very different histories (e.g. collision of two jets of debris). Tests were previously run using a variety of configurations (Gong, 2006; Lewellen et al., 2008), showing that simulation results were reasonably robust to the fluid approximation employed. Also, there is considerable uncertainty in choosing values for the surface parameters $c_{a}, c_{d}, c_{b}, c_{t}, c_{u}$, and debris availability level. No attempt has been made to correlate with any particular real surface; however the surface parameters and availability have been varied as tests of sensitivity (detailed in Chapter 3).

\subsection{Simulation procedures and default parameters}

Generally, a simulation was started by interpolating the flow field from a non-debris case onto a selected grid. The desired air flow boundary conditions and tornado translation speed were applied, and the simulation was run to a quasisteady state without debris to eliminate 
dependence on the initial conditions. If debris was desired, the simulation was stopped and the flow field regridded in the presence of a surface debris source. The simulation was then run to a new quasisteady state, allowing the debris cloud to develop self-consistently.

Constant angular momentum of $\Gamma_{\infty}=5000-15000 \mathrm{~kg} \mathrm{~m}^{2} \mathrm{~s}^{-1}$ was imposed on the lateral boundaries above a height of $10 \mathrm{~m}$. Below $10 \mathrm{~m}, \Gamma_{\infty}$ was in some cases set constant; in other cases $\Gamma_{\infty}$ was interpolated to zero to simulate depletion of near-surface angular momentum outside the simulation domain. Constant convergence of $0.010-0.015 \mathrm{~s}^{-1}$ was usually imposed all the way to the surface. Properties of the vortex aloft were sometimes tailored by applying a central downdraft of up to $w=15 \mathrm{~m} \mathrm{~s}^{-1}$ at the top boundary within a disk of radius $50-200 \mathrm{~m}$. The surface roughness length was set to $z_{0}=0.02 \mathrm{~m}$ (modeling a grassy lower boundary), and the surface-relative vortex translation speed was set within the range $U_{\text {trans }}=1-35 \mathrm{~m} \mathrm{~s}^{-1}$.

The majority of simulations employed an unlimited surface source comprised of a single, sand-like debris species with $\sigma / \rho \sim 2000$ and $d \sim 1 \mathrm{~mm}$. Such a debris type is representative of the fine soil expected to be present in the field (Soil Survey Staff, 1999). In select cases the amount of debris at the surface was set to $1 \mathrm{~kg} \mathrm{~m}^{-2}$ to explore the effects of limited debris availability. There is an insensitivity in the LES code (discussed in more detail in Section 3.4.3) that allows connections to be drawn between simulations of tornadoes carrying heavy, fine debris (e.g. sand) and tornadoes carrying light, large debris (e.g. corn stubble).

Most simulations were performed on a desktop computer with an 8-core Intel Xeon processor with clock speed $2.40 \mathrm{GHz}$; up to 14 simulation runs were executed simultaneously. Typically, a 150x150x113-point stretched grid was used to simulate a $2 \mathrm{~km}^{3}$ domain with finest central horizontal resolution of $5 \mathrm{~m}$ and finest vertical resolution of $1.0 \mathrm{~m}$ at the surface. This choice of grid structure provided adequate resolution of the most important flow features while not being prohibitive in terms of the processing times required. To solve the LES equations over $100 \mathrm{~s}$ of simulated time, it usually took a few hours of processing time in the absence of debris and about a day in the presence of one debris species, with 
the processing time increasing roughly linearly with the number of additional debris species. Debris types that experienced significant slip velocities in various portions of the flow tended to require higher processing times due to smaller timesteps forced by stability limits on the debris fields. Much finer central horizontal resolution (e.g. $2 \mathrm{~m}$ ) led to processing times of weeks to solve for $100 \mathrm{~s}$ of flow in the presence of one debris species, precluding generation of a large body of very high-resolution runs. 


\section{Chapter 3}

\section{Simulation of surface tracks}

Collections of simulated surface tracks are introduced in this chapter, the general physical origins of the most prominent surface marks are outlined, and sensitivities of the marks to simulation parameters are discussed. The simulation runs referred to in various figures are described in the Appendix.

\subsection{Surface track accounting and measures}

As implemented in the LES code utilized, separate tracks of cumulative removal and total deposition were collected by integrating respectively the upward and downward debris fluxes at the surface as the tornado moved over each surface track grid point (Lewellen et al., 2008). The physical signature is the difference between the two (i.e. deposition minus removal, the net debris deposition). Sample surface tracks of net deposition for two sets of tornadoes, with members moving at different translation velocities, are shown in Figures 3.1 and 3.2. Also provided for comparison are sample horizontal slices of the instantaneous fields responsible for the debris fluxes as they sweep over the surface and evolve in time. The appearances of the surface tracks are of primary concern here, but for reference the peak removal and deposition levels in the figures represent sand layer depths of order a few centimeters. Intensity levels have been chosen to clearly show prominent marks without 
saturation of the most important features. The sudden cutoff of the righthand portion of the track in Figure 3.1, panel (a) is simply due to the shorter length of track simulated relative to the tracks in panels $(\mathrm{b}, \mathrm{c})$.

Unless otherwise stated, tornado translation is taken to be to the right when viewed from above and tornado rotation is cyclonic (counterclockwise). For ease of discussion, the translation velocity vector $\vec{U}_{\text {trans }}$ (which is steady in time for all of the simulations) is taken to point toward the "front" of the tornado; the tornado's "left" and "right" would be to a ground-based observer's respective left and right when looking along $\vec{U}_{\text {trans }}$.

In addition to tracks of net sand removal/deposition, the LES code collects peak and timeintegrated measures of: debris flux to and from each surface point, upward and downward wind speeds at $\mathrm{z}=1.0 \mathrm{~m}$ height, ground-relative horizontal wind speeds at $\mathrm{z}=0.5 \mathrm{~m}$, pressure drop at the surface, and subgrid turbulent kinetic energy at $\mathrm{z}=0.5 \mathrm{~m}$. Samples of these fields for the most swiftly-translating runs in Figures 3.1 and 3.2 are shown in Figures 3.3-3.5.

Simulated directional markers have also been implemented in the LES code, recording the local windspeed vector at $0.5 \mathrm{~m}$ height at the first and last time it exceeds a given threshold (e.g. Figures 3.6 and 3.7). The former behave much like cornstalks that blow over and immediately become stuck to the ground, and the latter behave like cornstalks that remain rooted but change direction with the local wind until the tornado passes. No attempt has been made to correlate the "cornstalk" marker signals (or any other damage track field) with the potential for damage to man-made structures (e.g. the Enhanced Fujita scale (McDonald and Mehta, 2006)), as this requires assumptions about engineering and integrated time of interaction with a tornado. Simulated, stationary, surface probes have also been implemented to allow comparison between the time history of the flow and hydrodynamic data collected by in situ instrumentation in the field (e.g. Figure 3.8). 

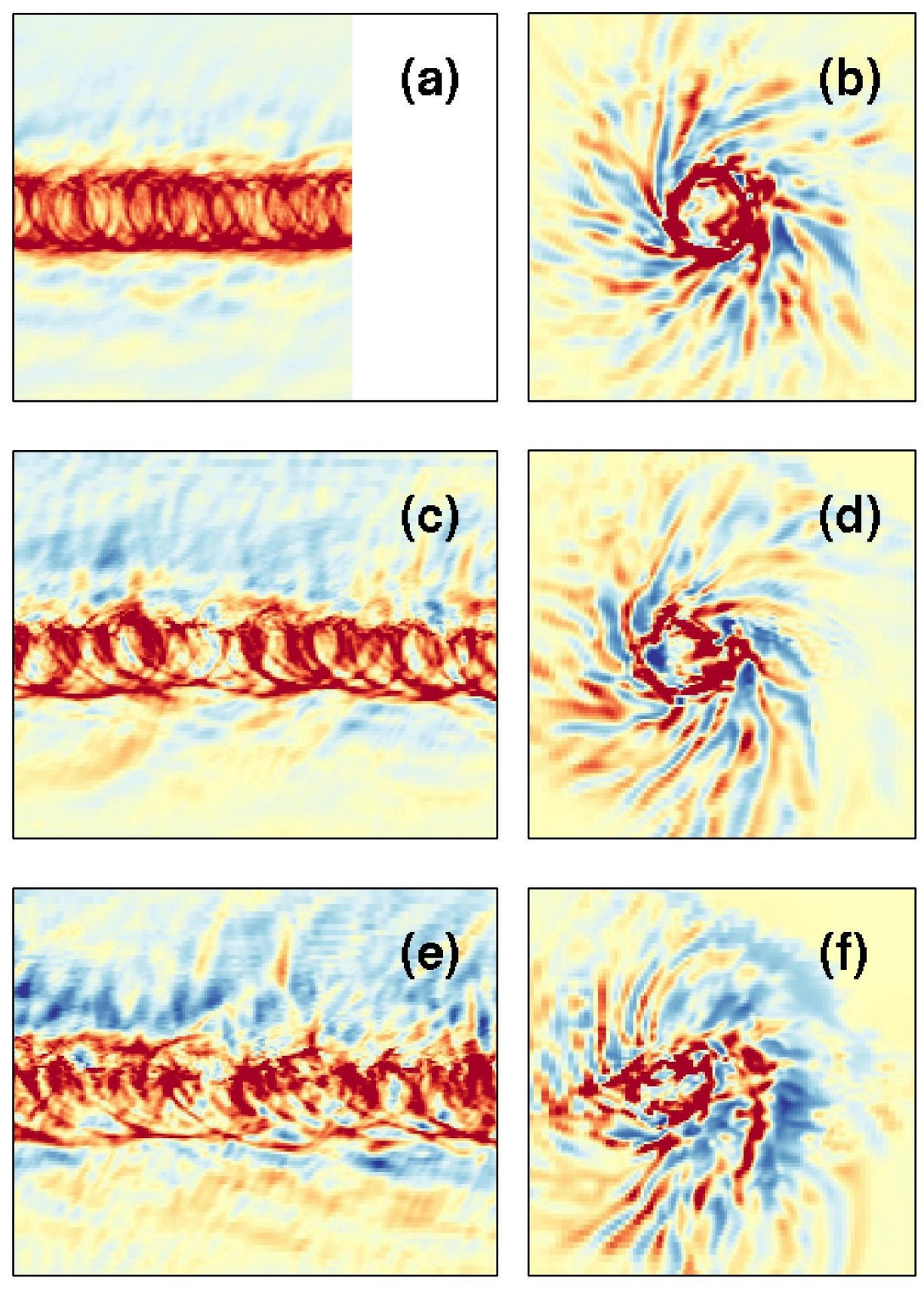

Figure 3.1: Representative surface tracks of net debris deposition (left column) and instantaneous debris flux at the surface (right column) for sample high-swirl tornadoes moving to the right at (a,b) $5 \mathrm{~m} / \mathrm{s},(\mathrm{c}, \mathrm{d}) 15 \mathrm{~m} / \mathrm{s}$, and (e,f) $25 \mathrm{~m} / \mathrm{s}$ (runs dmg2059, dmg2014, dmg2060, respectively). Cool colors represent net removal of debris while warm colors represent net deposition. Peak sand layer depths are on the order of a few $\mathrm{cm}$, with intensities scaled by $U_{\text {trans }}^{-1} / V_{c}$. The lateral dimensions are $800 \mathrm{~m}$. 

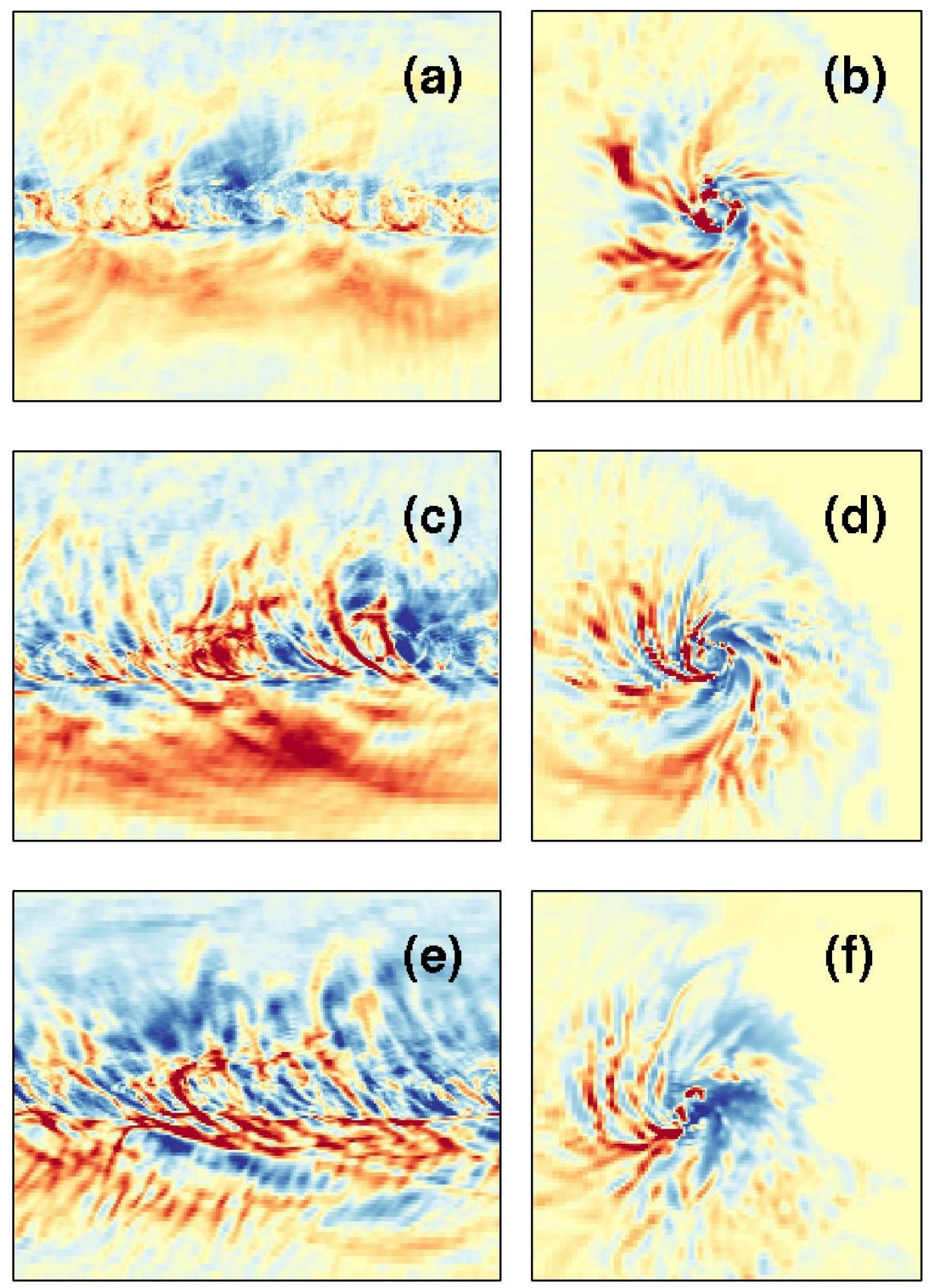

Figure 3.2: As in Figure 3.1 except for a sample of medium-swirl tornadoes. Note the transition here from the primarily cycloidal marks seen in Figure 3.1 to sets of longer, arcing marks and deposition of fully lofted debris to one side of the tornado. Runs are (a,b) dmg2049, (c,d) dmg2011, (e,f) dmg2052. 

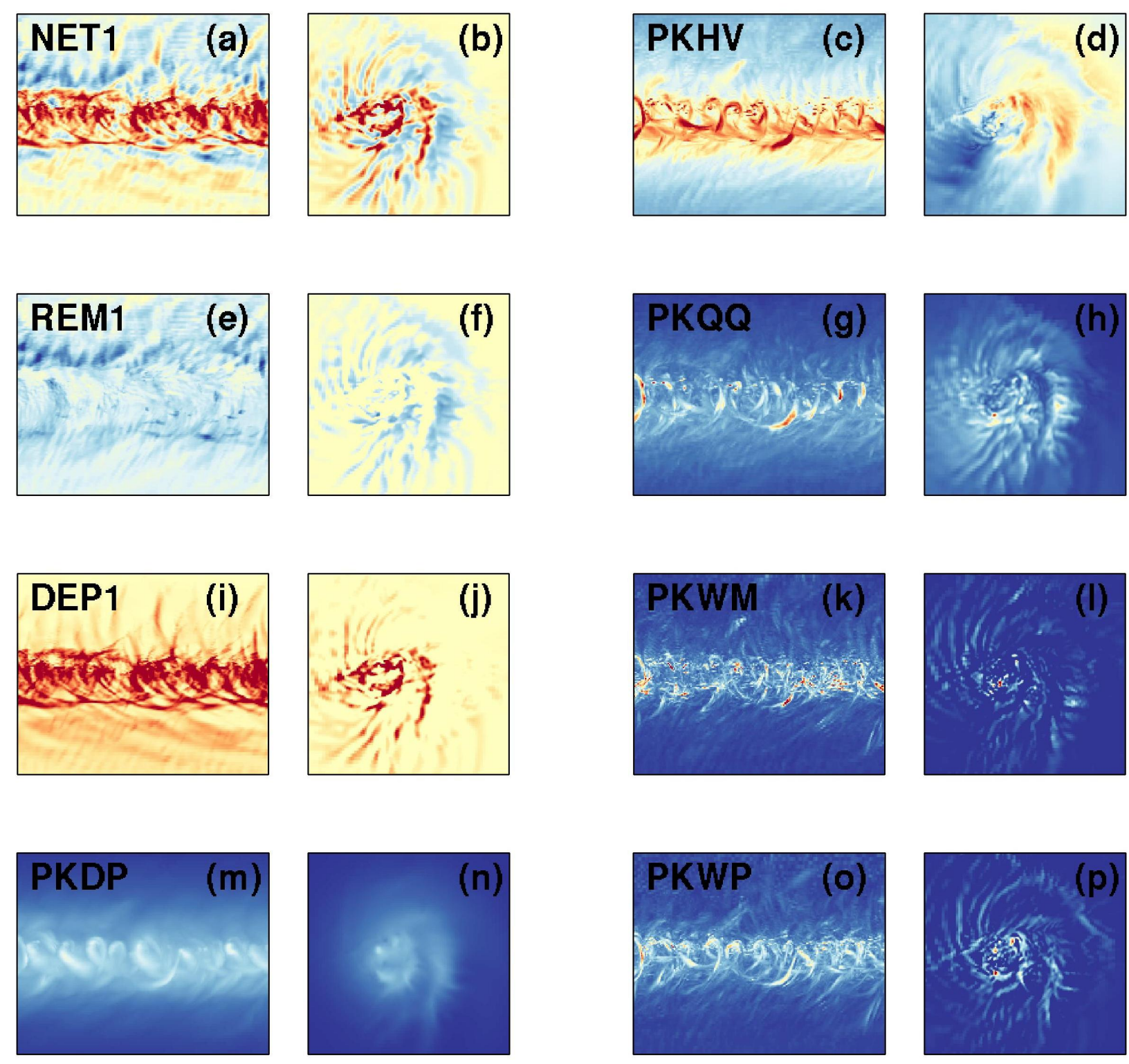

Figure 3.3: Deposition and peak measures for the case of Figure 3.1e,f (run dmg2060): (a,b) net deposition; (c,d) peak horizontal velocity at $\mathrm{z}=0.5 \mathrm{~m}$; (e,f) total removal; $(\mathrm{g}, \mathrm{h})$ peak subgrid turbulent kinetic energy at $\mathrm{z}=0.5 \mathrm{~m}$; $(\mathrm{i}, \mathrm{j})$ total deposition; $(\mathrm{k}, \mathrm{l})$ peak downward windspeed at $\mathrm{z}=1.0 \mathrm{~m} ;(\mathrm{m}, \mathrm{n})$ peak pressure drop at $\mathrm{z}=0.5 \mathrm{~m} ;(\mathrm{o}, \mathrm{p})$ peak upward windspeed at $\mathrm{z}=1.0 \mathrm{~m}$. 

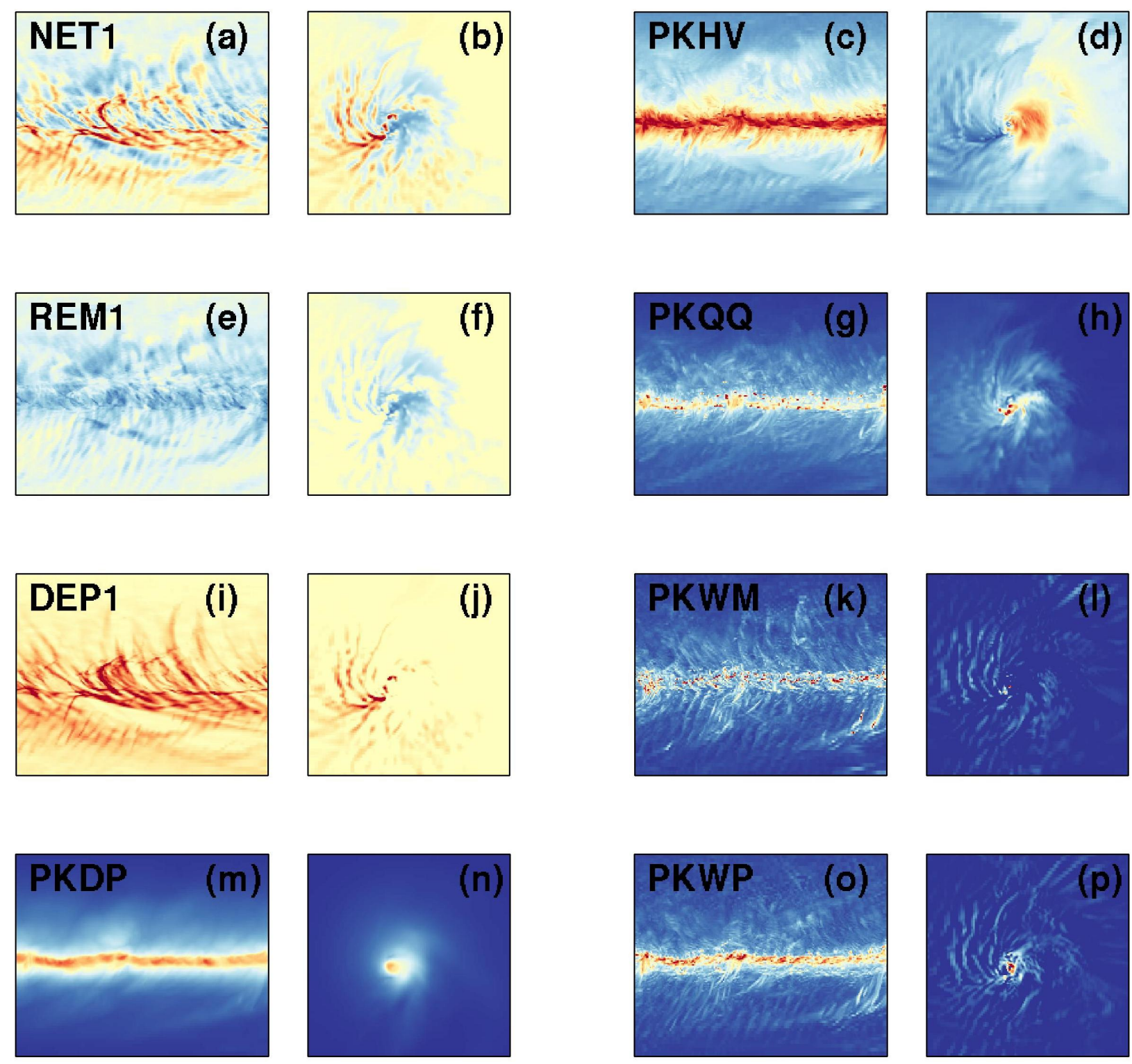

Figure 3.4: Deposition and peak measures as in Figure 3.3 except for the medium-swirl case in Figure 3.2e,f. 

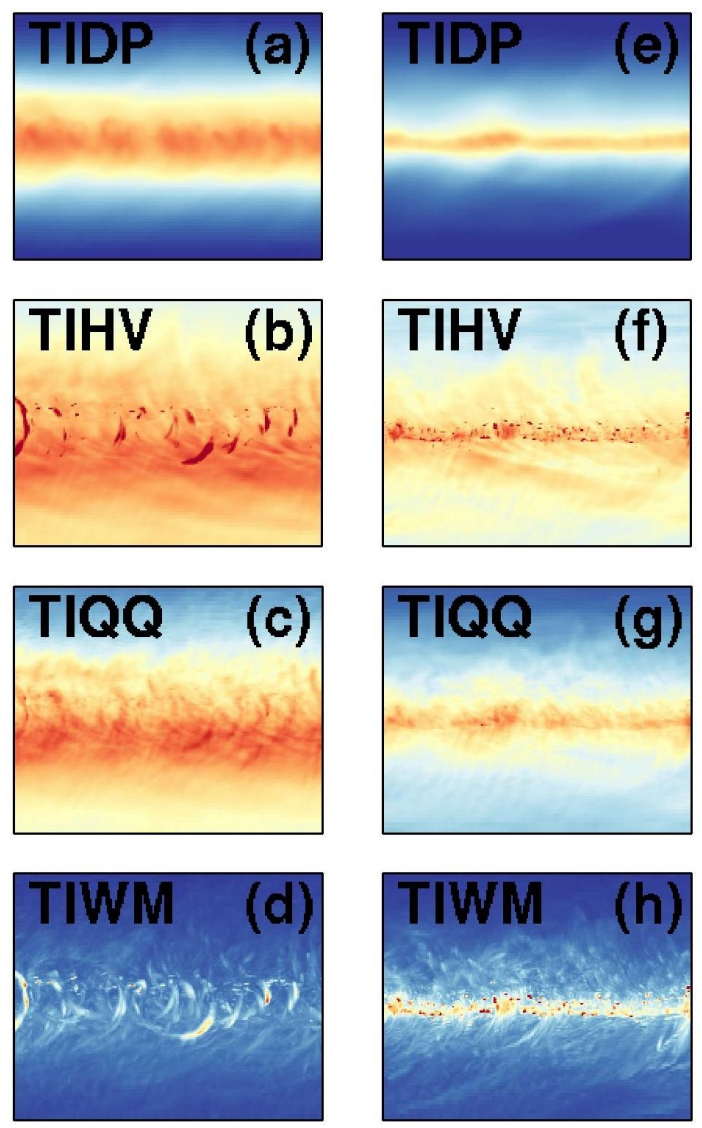

Figure 3.5: Time-integrated measures for (left column) the high-swirl case in Figure 3.1e,f (run dmg2060) and (right column) the medium-swirl case in Figure 3.2e,f (run dmg2052): (a) pressure drop at $\mathrm{z}=0.5 \mathrm{~m}$; (b) horizontal windspeed at $\mathrm{z}=0.5 \mathrm{~m}$; (c) subgrid turbulent kinetic energy at $\mathrm{z}=0.5 \mathrm{~m} ;(\mathrm{d})$ downward vertical windspeed at $\mathrm{z}=0.5 \mathrm{~m}$. 

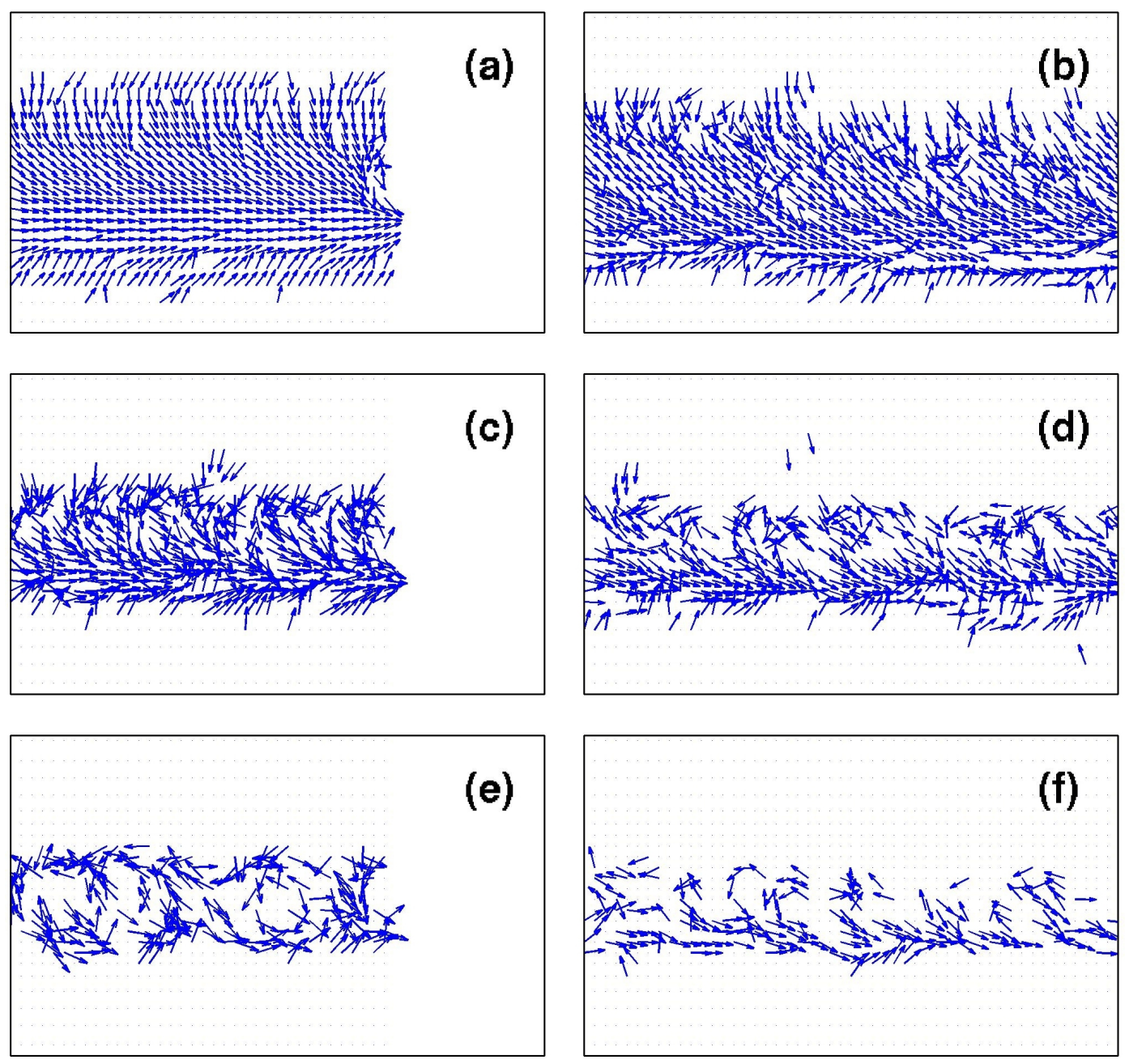

Figure 3.6: Directional wind markers taken at the first grid point above the surface for a collection of high-swirl tornadoes moving at (left column) $U_{\text {trans }}=5 \mathrm{~m} \mathrm{~s}^{-1}$ (run dmg2059) and (right column) $U_{\text {trans }}=25 \mathrm{~m} \mathrm{~s}^{-1}$ (dmg2060). The wind directions shown were taken at the last instant that the local surface-relative windspeed exceeded (top row) $29.1 \mathrm{~m} \mathrm{~s}^{-1}$, (middle row) $38.0 \mathrm{~m} \mathrm{~s}^{-1}$, and (bottom row) $49.2 \mathrm{~m} \mathrm{~s}^{-1}$ commensurate with EF-scale levels 1, 2, and 3. Wind directions taken at the first instant that wind thresholds were exceeded do not look greatly different than the plots shown here and in Figure 3.7. 

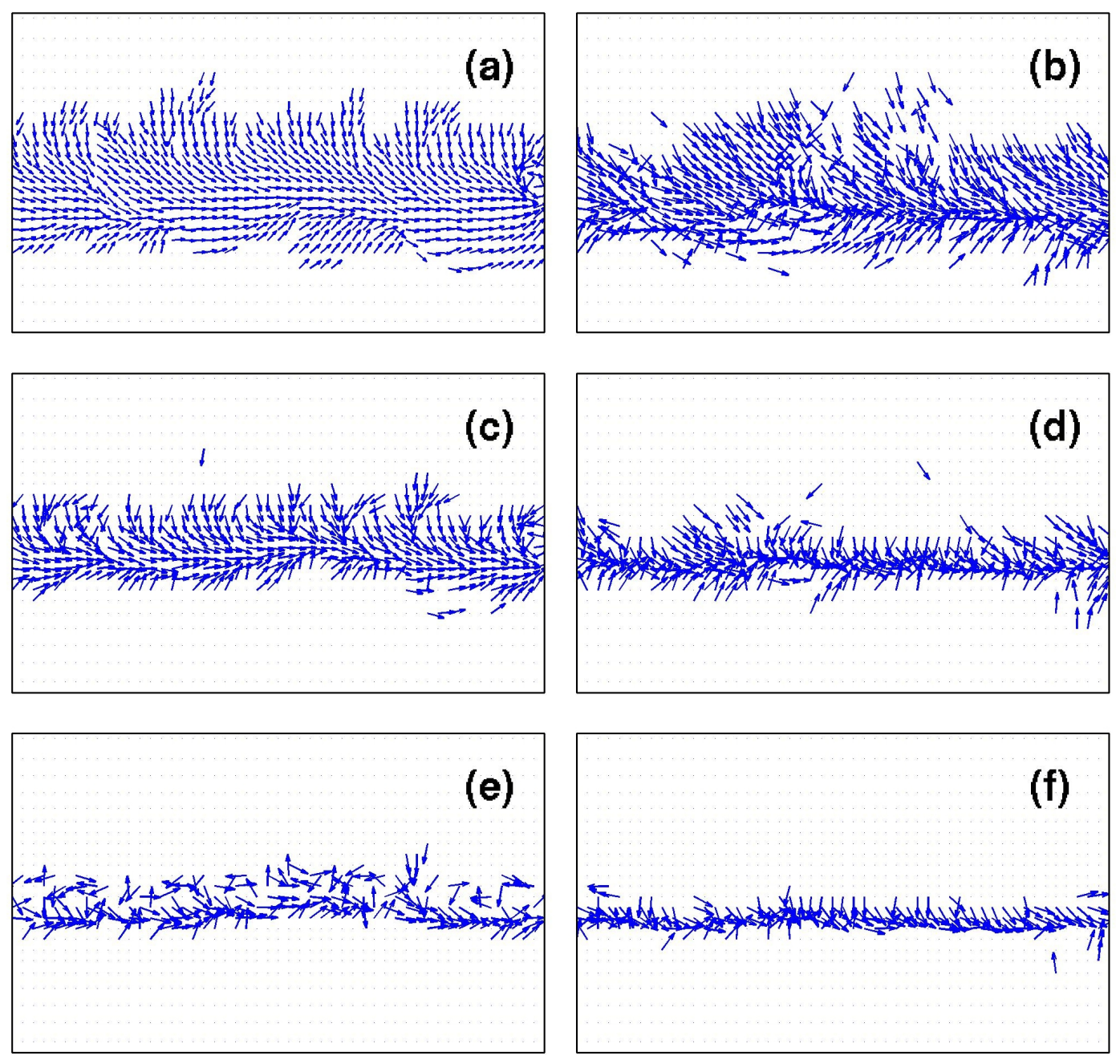

Figure 3.7: As in Figure 3.6, except for a collection of medium-swirl tornadoes. Left column: run dmg2049, moving at $5 \mathrm{~m} \mathrm{~s}^{-1}$; right column: run dmg2052, moving at $25 \mathrm{~m} \mathrm{~s}^{-1}$. 

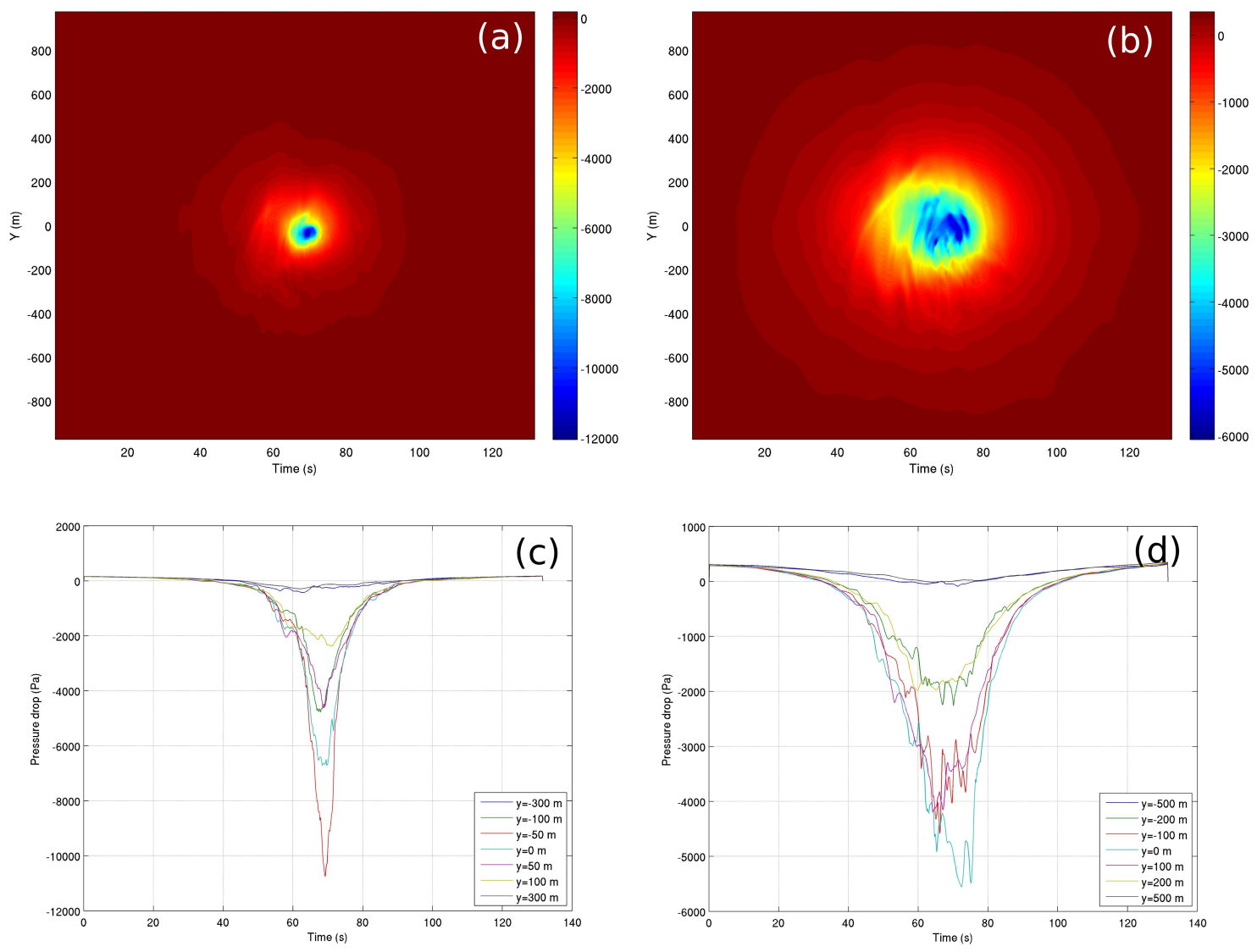

Figure 3.8: Top row: simulated time-dependent pressure traces taken by an array of stationary probes at $z=0.5 \mathrm{~m}$ (pressure measurements are in units of $\mathrm{Pa}$ ). Bottom row: sample traces from individual probes at varying lateral $y$-location. Panels $(\mathrm{a}, \mathrm{c})$ show data from a medium-swirl case (run dmg2011); panels (b,d) show data from a high-swirl case (run dmg2014). 


\subsection{General origins of surface marks}

There are five identifiable components to the surface debris flux: deposition and removal in the inflow layer, deposition and removal in the interior corner flow, and deposition of fully lofted debris. Each is responsible for different features of the debris tracks produced, with their relative intensities depending on tornado type, debris properties, and surface properties. Interpretation of surface marks is complicated because they are produced by a convolution of events in time and space, for example patches of removal towards the front of the tornado being overrun subsequently by patches of deposition. In general, it was found that the origin of even some very pronounced marks can involve the interaction of several factors and therefore change significantly and in complicated fashion as cases are varied.

Removal signatures generally correlate with subgrid turbulent kinetic energy, as expected from the debris pickup parameterization (cf. Equation 2.14); this claim is supported by the similarities in patterns of net removal and peak subgrid turbulence intensity in Figures 3.3e-h and 3.4e-h. Contrary to historical interpretations of surface marks, secondary vortex paths were found not to correlate strongly with intense deposition marks (e.g. compare the net deposition and peak pressure drop tracks in Figures 3.3a,m and 3.4a,m). Similar comparisons between net deposition patterns and the other measures in Figures 3.3-3.5 reveal that the strongest net deposition signatures do not, in general, correlate well with any of the peak or time-integrated measures collected.

The most prominent central deposits are often from debris left behind when flow turns sharply upward in the corner flow region, typically in a loose annulus or arc (e.g. the central deposition marks in Figures 3.1b,d,f). Finer-scale alternating deposition and removal marks away from the central region are typically traceable to patterns of inflow rolls in the surface layer and are often more prominent for higher vortex translation speeds (e.g. the long, arcing deposition swaths in Figure 3.2c,e; see also Figure 5.19). More extensive swaths of deposition away from the center are typically from lofted debris (e.g. the diffuse deposition marks found to the tornado's right in Figure 3.2a,c). 


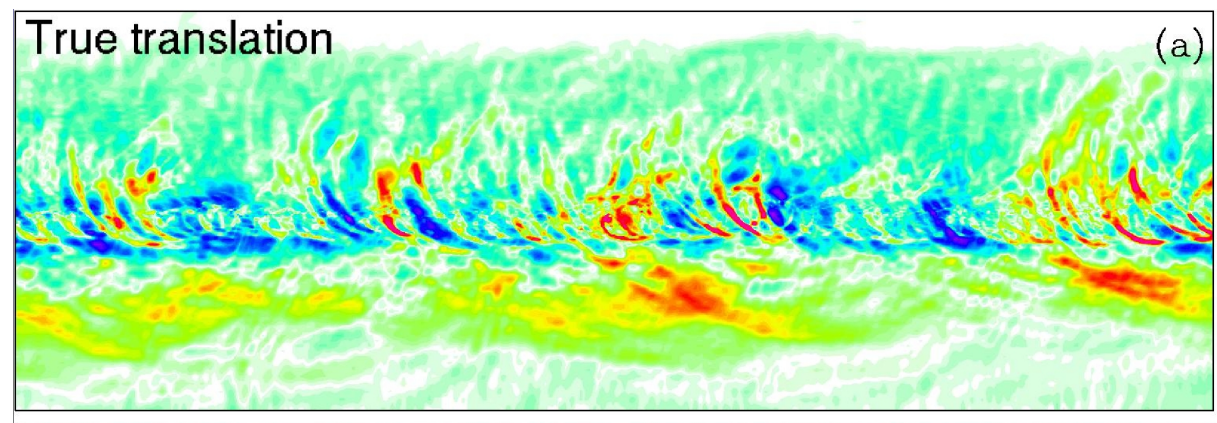

Artificial translation of non-translating tornado

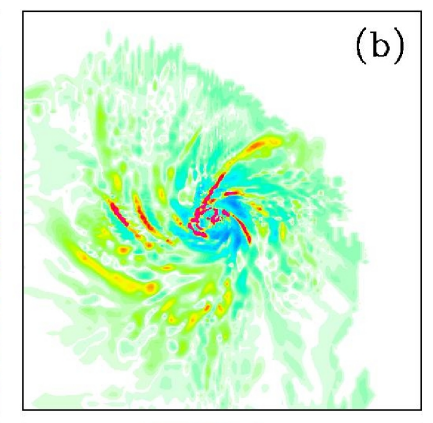

(d)
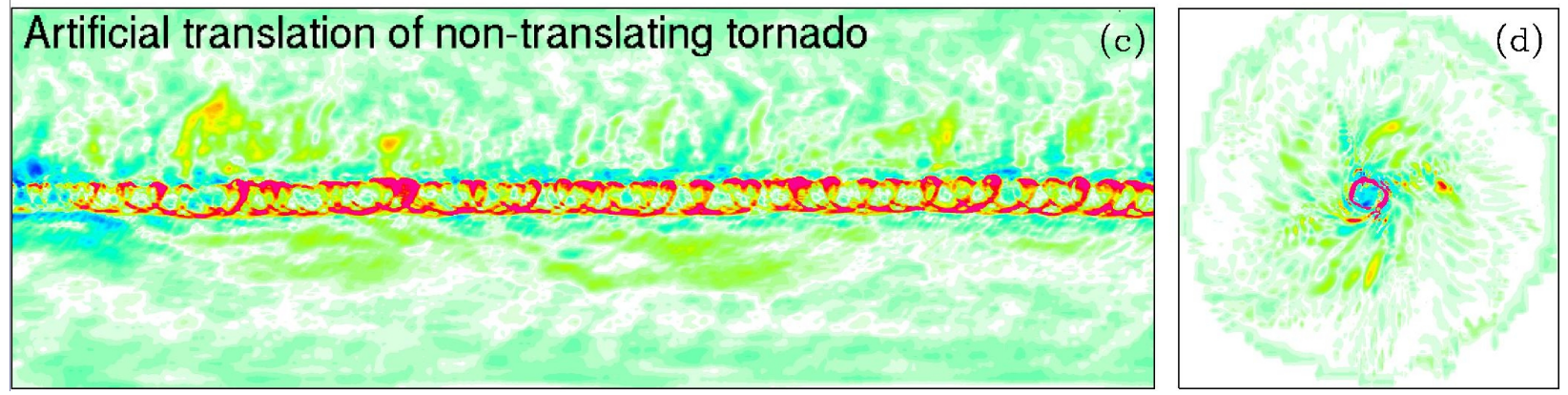

Figure 3.9: Surface deposition/removal tracks (panels a,c) and sample instantaneous debris fluxes to the surface $(\mathrm{b}, \mathrm{d})$ for two idealized medium-swirl simulation cases. In (c,d) a surface track is artificially collected from a non-translating (and therefore axisymmetric in the mean) tornado as if it were moving at $15 \mathrm{~m} / \mathrm{s}$; otherwise this case can be directly compared with that in $(a, b)$, which is also shown in Figure 3.2. From Lewellen and Zimmerman (2008).

Asymmetries in the near-surface flow, such as those from the vortex translation, play a critical role in the appearance of the simulated tracks (see, for example, Figure 3.9). The addition of translation to a cyclonic vortex has several effects. In the far-field it can lead to more removal on the right where the swirl and translation velocities add. However, it also tends to tilt the vortex (typically right and rearward near the surface relative to the vortex center aloft) which can lead to greater removal to the left and front. The accompanying tilt of the debris cloud leads to asymmetric deposits of lofted debris, typically right and rearward. It affects the appearance of the central deposition by making the corner flow updraft just off the surface less symmetric, and it often produces trains of debris-laden inflow rolls that deposit debris behind the central vortex. 


\subsection{Turbulent variability of surface tracks}

Some deposition tracks are qualitatively uniform over a great length, as in Figure 3.10a. In this case a relatively short length of track (i.e. only one or two times $R_{c}$ aloft) is adequate to estimate the radius and shift of cycloidal marks relative to the flow aloft and to get a qualitative sense of the spacings between marks and the complete set of marks present. Although turbulent surface rolls modulate the intensity of deposition and removal as they sweep through the extended inflow region (e.g. see Figure 3.1b), their time-integrated signature is one of overall removal. The near surface deposition pattern shows some variability under the central vortex, where the strongest marks are laid down (e.g. Figure 3.11); however, the resulting set of marks in Figure 3.10a is quite uniform in character.

On the other hand, some tracks exhibit a considerable amount of variability as the tornado moves along, as in Figure 3.10b. It can be seen from the corresponding time progression of surface debris flux in Figure 3.12 that deposition under the central vortex, deposition by surface rolls trailing the main vortex, and deposition of lofted debris all fluctuate over time, competing to create the strongest marks. One would require a long portion of the track (i.e. perhaps 5-10 $R_{c}$ ) to draw conclusions about the full population of marks or at least to capture a few distinct marks for analysis.

The key point is that the most complete information is carried by sets of marks over some length of track rather than individual marks taken alone.

\subsection{Sensitivities to simulation parameters}

\subsubsection{Sensitivities to surface parameterization}

There is considerable uncertainty in the parameterization of the debris pickup off the surface, much of it reflecting real physical variability in conditions (e.g., soil moisture content, debris shape, presence of vegetation, debris availability, etc.). However, the sensitivity to 

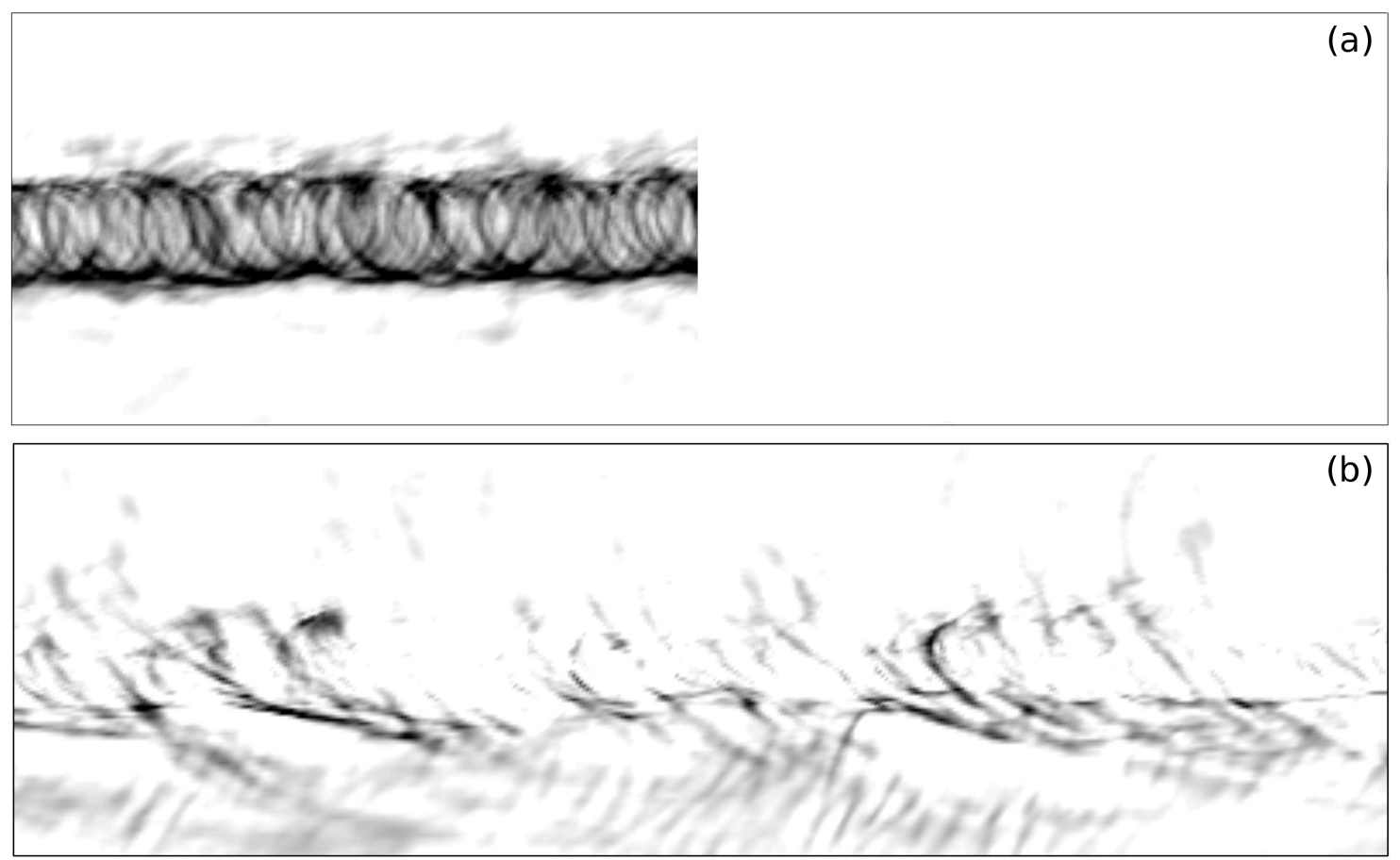

Figure 3.10: Deposition tracks from (a) the high-swirl case from Figure 3.1a (dmg2059) and (b) the medium-swirl case from Figure 3.2e (run dmg2052), where only the positive parts of the net deposition signals have been plotted. Comparison of (a) and (b) demonstrates differences in turbulent variability between tracks produced by different simulated tornadoes. Lateral dimensions are $600 \mathrm{~m}$; the vortex diameter aloft is about (a) $400 \mathrm{~m}$ and (b) $220 \mathrm{~m}$. White regions map to $0 \mathrm{~kg} \mathrm{~m}^{-2}$ and the darkest regions map to (a) $130 \mathrm{~kg} \mathrm{~m}^{-2}$ and (b) 30 $\mathrm{kg} \mathrm{m}^{-2}$. 

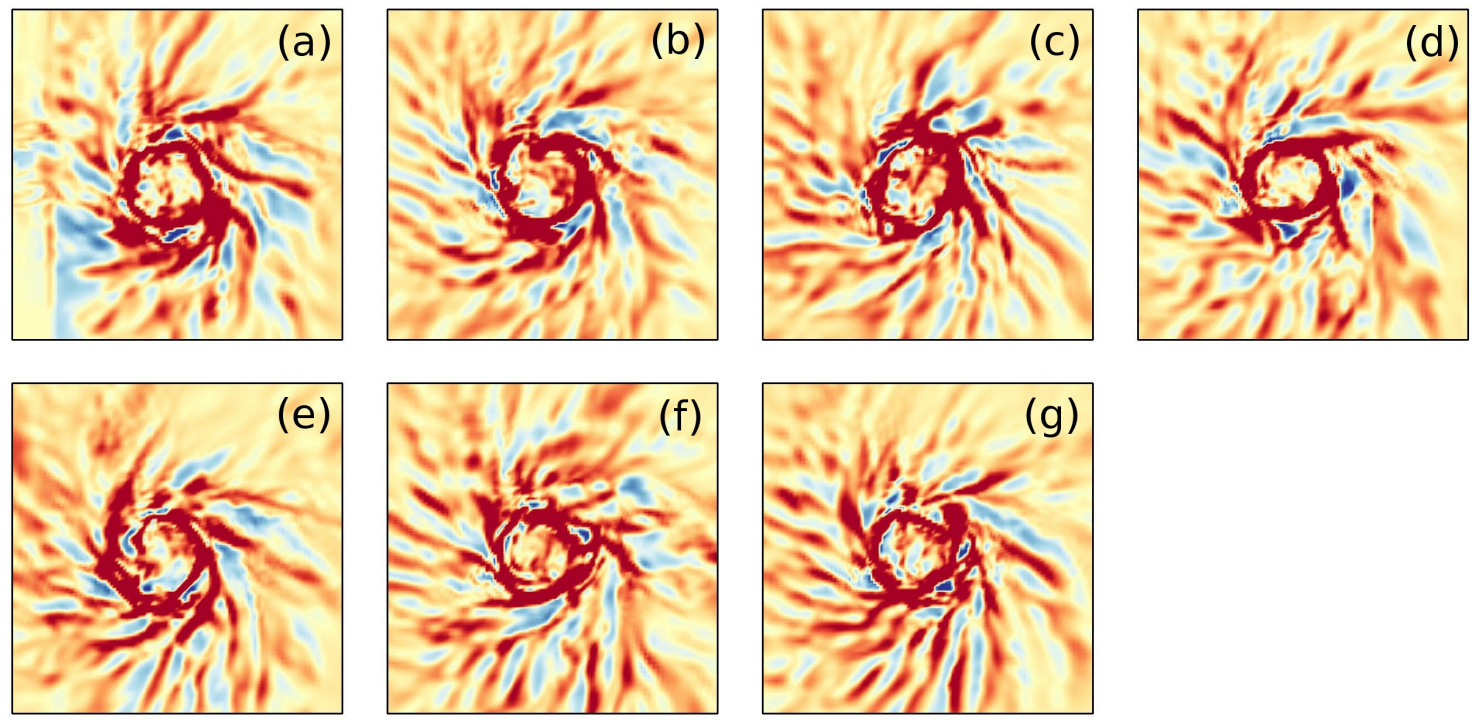

Figure 3.11: Instantaneous snapshots of vertical debris flux at the surface at $150 \mathrm{~m}$ intervals across the track in Figure 3.10a (run dmg2059), showing little turbulent variability. Deep red/blue correspond respectively to flux levels of $+/-2 \mathrm{~kg} \mathrm{~m}^{-2} \mathrm{~s}^{-1}$. The plot dimensions are $600 \mathrm{~m}$.
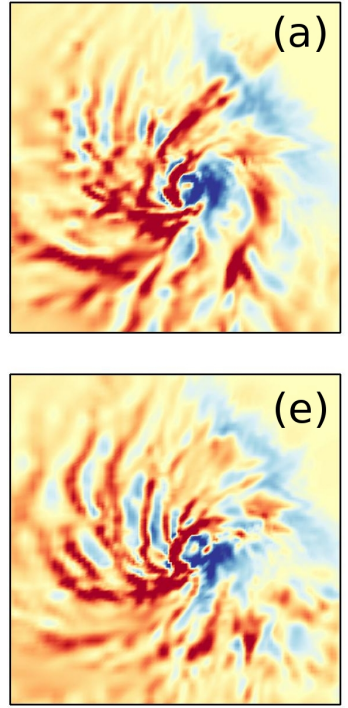
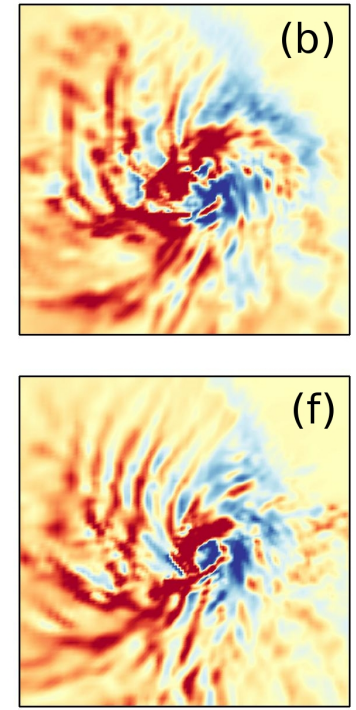
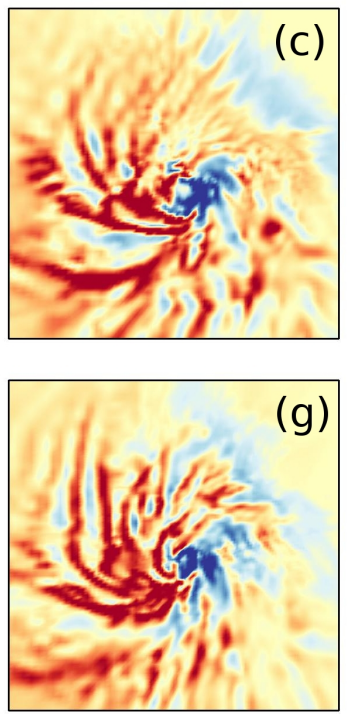
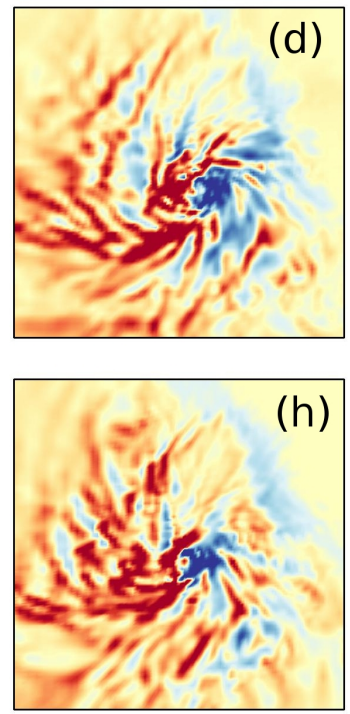

Figure 3.12: As in Figure 3.11, except for the track in Figure 3.10b (run dmg2052), showing some turbulent variability. Deep red/blue correspond respectively to flux levels of $+/-2 \mathrm{~kg}$ $\mathrm{m}^{-2} \mathrm{~s}^{-1}$. The plot dimensions are $600 \mathrm{~m}$, and in order to show the most important flow features the area plotted trails the vortex center aloft by $100 \mathrm{~m}$ (with respect to the direction of vortex translation). 
surface conditions is reduced by a negative physical feedback between near-surface debris removal and mass loading, as described in Section 1.2. Surface parameters have been varied as tests of sensitivity but no attempt has been made to correlate any particular choices with specific natural surfaces.

The parameters varied are those controlling the relative importance of different contributions to the vertical debris flux at the surface (c.f. Equations 2.14-2.16): $c_{a}$ (controlling the amount of debris removed by local subgrid turbulence), $c_{d}$ (controlling the amount of debris removed by collisions with lofted debris), and $c_{t}$ (the wind threshold for removal of debris from the surface), $c_{b}$ (controlling the amount of debris that sticks upon impact), and $c_{u}$ (controlling the amount of horizontal momentum transferred to the surface during debris impacts).

\section{Variation of tracks with $c_{a}$}

Figures 3.13 and 3.14 show deposition and instantaneous debris flux patterns for a collection of tornadoes with $c_{a}$ ranging from 0.04 to 0.36 . Increasing $c_{a}$ causes more debris to be removed by a given wind level; indeed, absolute total deposition levels followed along with $c_{a}$ among each swirl class represented in Figures 3.13 and 3.14, as did the total debris loadings aloft (e.g. Table 3.1).

In the medium-swirl regime, raising $c_{a}$ favored deposition underneath the central vortex over deposition in the far field. Though the total debris cloud mass grew with $c_{a}$, the amount of debris lofted relative to the total removed decreased with $c_{a}$ (e.g. Table 3.1). These observations are consistent with a weakened near-surface radial inflow due to enhanced debris loading, leading to a weakened central updraft and ultimately causing a larger fraction of debris to be left behind underneath the corner flow upturn. The medium-swirl track at $c_{a}=0.36$ (e.g. Figure 3.13e) qualitatively resembles the high-swirl track at the same value of $c_{a}$ (e.g. Figure 3.13f), with central, cycloidal-like deposition flanked by signatures of inflow rolls; this is consistent with increased effective $S_{c}$. 

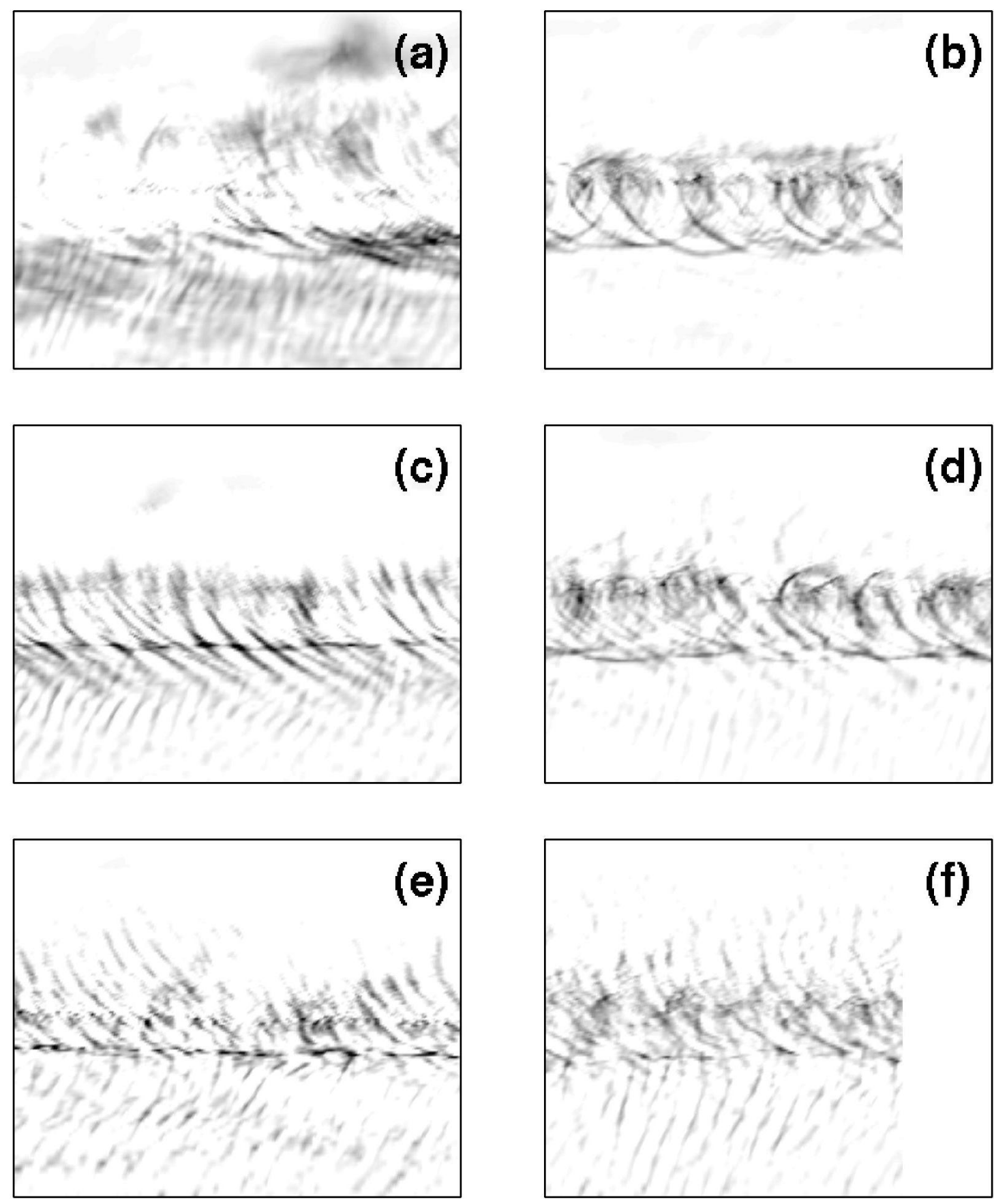

Figure 3.13: Deposition tracks from simulated medium- and high-swirl tornadoes (left and right columns, respectively) with different removal parameter $c_{a}$. Top to bottom: $c_{a}=$ $0.04,0.12,0.36$. Grayscales are multiplied by $c_{a} / 0.12$ to provide similar intensities across the entire set. The darkest shade of gray in the middle row corresponds to deposition levels of (left column) $30 \mathrm{~kg} \mathrm{~m}^{-2}$ and (right column) $60 \mathrm{~kg} \mathrm{~m}^{-2}$. Lateral dimensions are $800 \mathrm{~m}$. Simulation runs are (a) dmg2274, (b) dmg2277, (c) dmg3026, (d) dmg2082, (e) dmg2247, (f) dmg2276. 

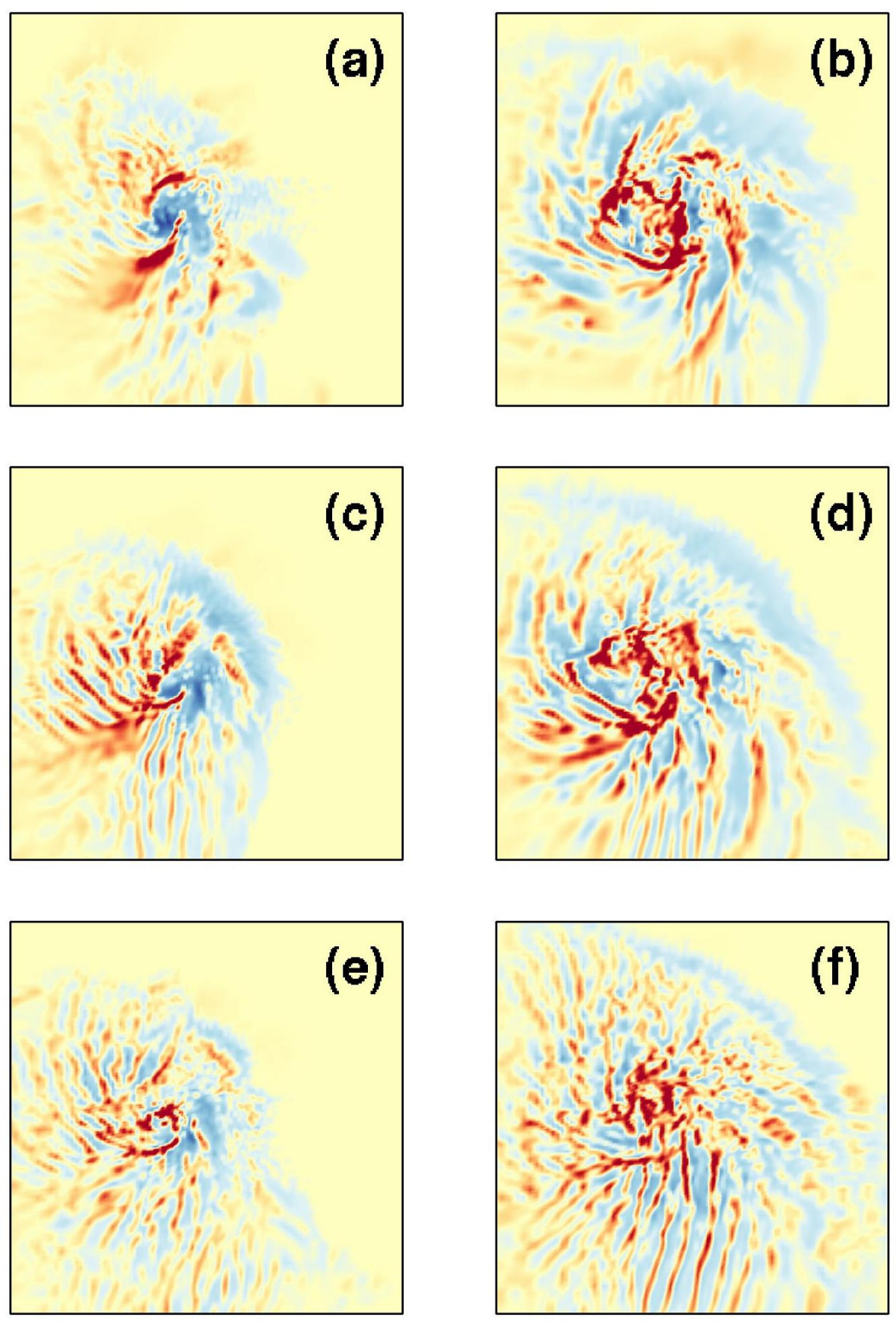

Figure 3.14: Instantaneous snapshots of surface debris flux for the cases in Figure 3.13 (panels a-f in this Figure correspond respectively to panels a-f in Figure 3.13).. Colorscales are multiplied by $c_{a} / 0.12$ to provide similar contrast between deposition and removal across the entire set. The deepest shades of red/blue in the middle row correspond to surface debris fluxes of $+/-3 \mathrm{~kg} \mathrm{~m}^{-2} \mathrm{~s}^{-1}$. Lateral dimensions are $800 \mathrm{~m}$. 


\begin{tabular}{|c|cc|ccc|}
\hline Name & $c_{a}$ & $S_{c}$ & $\begin{array}{c}\text { Deposition per length } \\
\left(\mathrm{kg} \mathrm{m}^{-2} \mathrm{~m}^{-1}\right)\end{array}$ & $\begin{array}{c}\text { Loading above } 10 \mathrm{~m} \\
\left(\times 10^{6} \mathrm{~kg}\right)\end{array}$ & $\begin{array}{c}\text { Loading below } 10 \mathrm{~m} \\
\left(\times 10^{6} \mathrm{~kg}\right)\end{array}$ \\
\hline dmg2274 & 0.04 & 3.0 & 0.08 & 0.97 & 0.37 \\
dmg3026 & 0.12 & 3.0 & 0.15 & 1.71 & 1.31 \\
dmg2247 & 0.36 & 3.0 & 0.39 & 2.58 & 4.62 \\
\hline dmg2277 & 0.04 & 12.4 & 0.08 & 0.59 & 0.68 \\
dmg2082 & 0.12 & 12.4 & 0.26 & 1.01 & 2.28 \\
dmg2276 & 0.36 & 12.4 & 0.83 & 1.41 & 7.26 \\
\hline
\end{tabular}

Table 3.1: Statistics on debris loading and net deposition under different values of the surface parameter $c_{a}$ (from the runs represented in Figure 3.13).

\section{Variation of tracks with $c_{d}$ and $c_{t}$}

Figures 3.15 and 3.16 show deposition patterns and debris fluxes for a collection of tornadoes with $c_{d}=0.00, c_{t}=0.50$ and $c_{d}=0.30, c_{t}=2.0$. Increasing $c_{d}$ enabled more debris to be released by collisions with lofted debris; however, simultaneously increasing $c_{t}$ raised the lofting threshold enough to overcome the effects of $c_{d}$. The most prominent changes between the two sets of tracks are in the lateral extent of removal (cf. contours of removal in Figure 3.15a,c). Near-surface winds far from the central vortex were rendered incapable of removing far-field deposits due to increased $c_{t}$ (e.g. the appearance of left-hand deposits in Figure $3.15 \mathrm{c}, \mathrm{d})$. Otherwise, the tracks were largely insensitive to the modest changes imposed on $c_{d}$ and $c_{t}$; the medium-swirl cases both exhibited evidence of deposition by inflow rolls, and the high-swirl cases contained cycloids with roughly the same lateral extent and apparent spacing.

\section{Variation of tracks with $c_{b}$}

Figure 3.17 shows deposition patterns and debris fluxes for a collection of tornadoes with $c_{b}$ ranging from 0.25 to $1.00 . c_{b}$ controls the fraction of debris that sticks upon impact. The most striking change when lowering $c_{b}$ from its default value of 1.00 to 0.25 (thus decreasing the fraction of debris that sticks to the surface upon impact from $100 \%$ to $25 \%$ ) is that the prominent marks become "smeared out" across the surface. Also, the alternating up/down debris fluxes that lead to arc-like features in the track of Figure 3.17a are strongly suppressed 

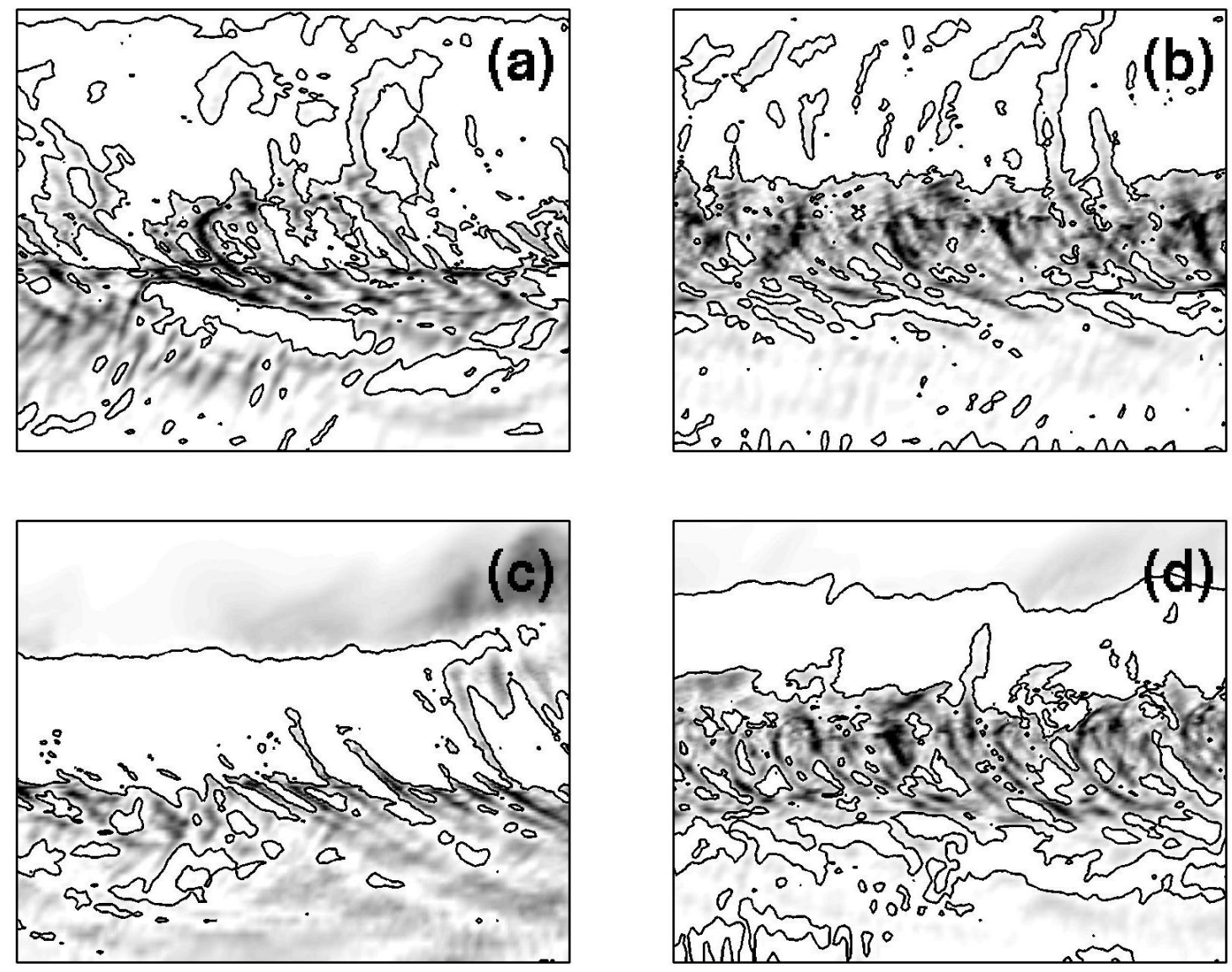

Figure 3.15: Sample surface tracks for (left column) medium- and (right column) high-swirl tornadoes with different surface parameters $c_{d}$ and $c_{t}$ (top: $c_{d}=0.00, c_{t}=0.50$, bottom: $\left.c_{d}=0.30, c_{t}=2.0\right)$. Lateral dimensions are $800 \mathrm{~m}$ and the darkest shades of gray correspond to a deposition level of (left column) $30 \mathrm{~kg} \mathrm{~m}^{-2}$ and (right column) $60 \mathrm{~kg} \mathrm{~m}^{-2}$. Contours correspond to a removal level of $2 \mathrm{~kg} \mathrm{~m}^{-2}$; the lateral spatial extent of removal decreases with increasing $c_{t}$. Simulation runs are (a) dmg2052, (b) dmg2060, (c) dmg3306, (d) dmg2042. 

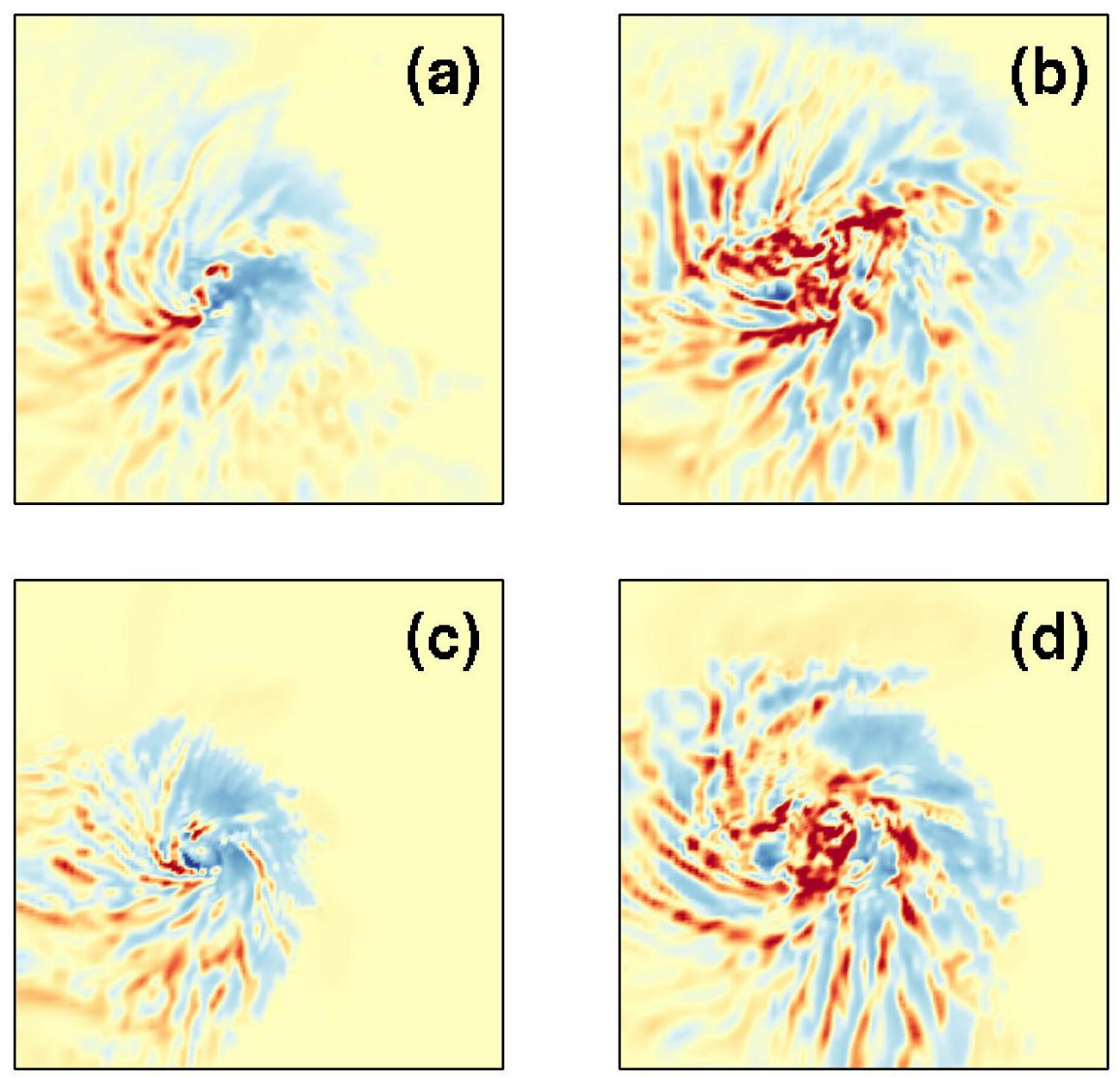

Figure 3.16: Sample instantaneous surface debris fluxes for the tracks in Figure 3.15. Lateral dimensions are $800 \mathrm{~m}$ and the deepest shades of blue/red correspond to debris flux levels of $+/-3 \mathrm{~kg} \mathrm{~m}^{-2}$. 


\begin{tabular}{|c|ccc|ccc|}
\hline Name & $c_{b}$ & $c_{u}$ & $S_{c}$ & $\begin{array}{c}\text { Deposition per length } \\
\left(\mathrm{kg} \mathrm{m}^{-2} \mathrm{~m}^{-1}\right)\end{array}$ & $\begin{array}{c}\text { Loading above } 10 \mathrm{~m} \\
\left(\times 10^{6} \mathrm{~kg}\right)\end{array}$ & $\begin{array}{c}\text { Loading below } 10 \mathrm{~m} \\
\left(\times 10^{6} \mathrm{~kg}\right)\end{array}$ \\
\hline dmg2052 & 1.00 & 1.00 & 3.0 & 0.15 & 1.71 & 1.31 \\
dmg2283 & 0.25 & 1.00 & 3.0 & 0.15 & 2.14 & 4.01 \\
\hline dmg2082 & 1.00 & 1.00 & 12.4 & 0.26 & 1.01 & 2.28 \\
dmg2282 & 0.25 & 1.00 & 12.4 & 0.31 & 1.25 & 6.48 \\
\hline
\end{tabular}

Table 3.2: Statistics on debris loading and net deposition under different values of the surface parameter $c_{b}$ (from the runs represented in Figure 3.17). Debris loadings are taken above and below $10 \mathrm{~m}$, which in these cases effectively separates the debris populations that do and do not follow the corner flow aloft.

at lower $c_{b}$ in the track of Figure 3.17c. Decreasing $c_{b}$ by a factor of 4 between the mediumswirl test cases (dmg2052/dmg2283) increased the near-surface debris loading by almost a factor of 4 while leaving the mean deposition per unit length unchanged (e.g. Table 3.2).

Overall, the tracks exhibited sensitivity to $c_{b}$. However, cycloidal marks and signatures of inflow rolls created in the parameter regime where $c_{b} \cong 1$ will likely be largely undetectable in the field due to their more-diffuse character. Linear signatures like that in the mediumswirl track of Figure 3.17 may be detectable in the field; however, they are similar to linear signatures in other medium-swirl cases (e.g. Figure 5.7) and may still provide a qualitative indication of the tornado type. Also, it is unclear that the low- $c_{b}$ limit is physical in that any real, statistically rough surface will contain obstructions to bouncing debris (e.g. leafy crops and variations in the local slope of the surface), the surface may be effectively sticky from rain (e.g. mud), and even a heavily debris-laden, dry surface (e.g. a sand pit) will have $c_{b}$ near 1 .

\subsubsection{Grid resolution}

Sensitivity checks were performed to explore the effects of grid resolution on the appearance of tracks. Figures 3.20 and 3.19 show surface tracks for two collections of tornadoes with $S_{c}=3.0$ and $S_{c}=12.4$ with different horizontal grid resolution across a region at the center of the simulations.

In the high-swirl regime (e.g. Figure 3.20) changes in central grid resolution have little 

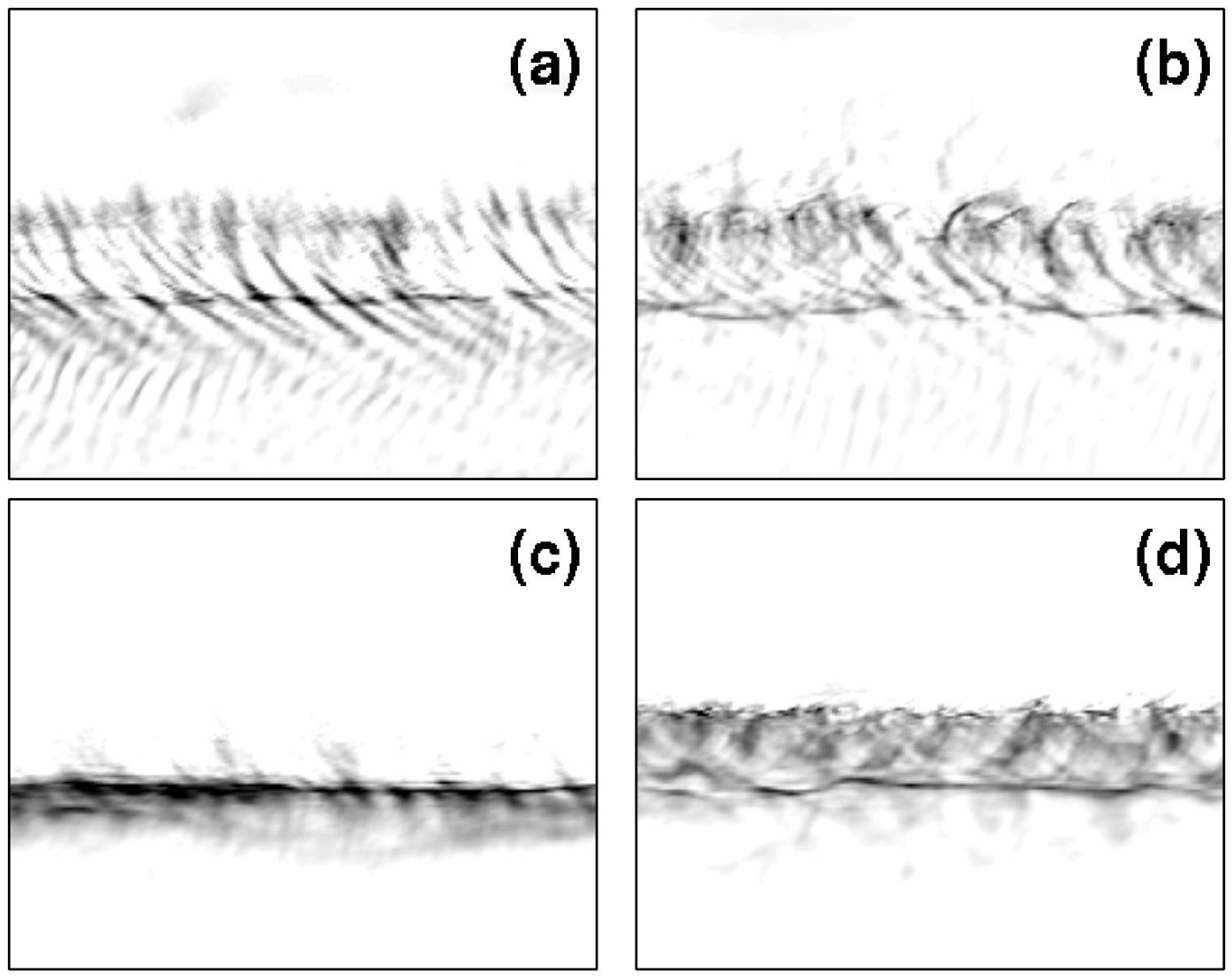

Figure 3.17: Sample surface tracks for (left column) medium- and (right column) high-swirl tornadoes with different surface parameter $c_{b}$ (top: $c_{b}=1.00$, bottom: $c_{b}=0.25$ ). Lateral dimensions are $800 \mathrm{~m}$ and the darkest shades of gray correspond to a deposition level of (left column) $30 \mathrm{~kg} \mathrm{~m}^{-2}$ and (right column) $60 \mathrm{~kg} \mathrm{~m}^{-2}$. Simulation runs are (a) dmg3026, (b) dmg2082, (c) dmg2283, (d) dmg2282. 

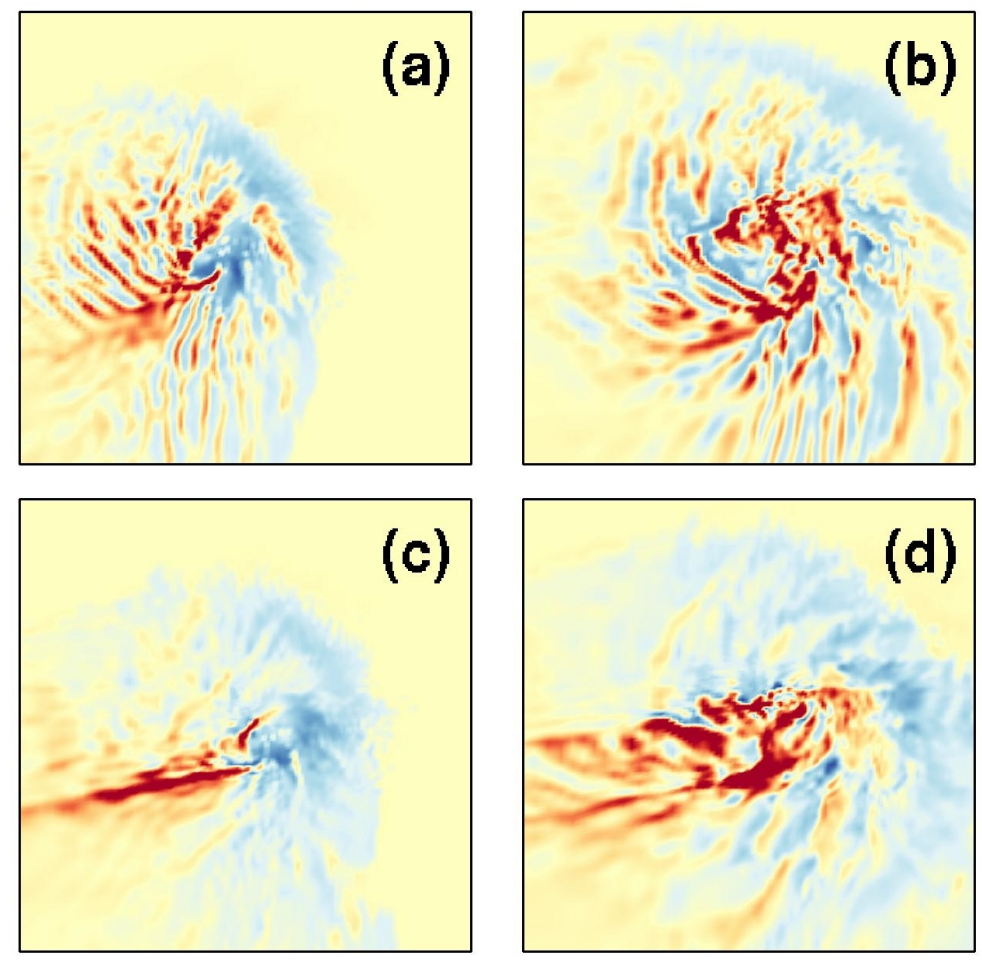

Figure 3.18: Sample instantaneous surface debris fluxes for the tracks in Figure 3.17. Lateral dimensions are $800 \mathrm{~m}$ and the deepest shades of blue/red correspond to debris flux levels of $+/-3 \mathrm{~kg} \mathrm{~m}^{-2} \mathrm{~s}^{-1}$. 
effect on the types and intensities of marks populating the tracks. At higher resolution, finerscale detail becomes evident and frequent arcs can be seen in the far field in faster-moving cases. The extra fine-scale features imply a higher cycloidal multiplicity; however, the most prominent marks occur at approximately the same spacing as those in lower-resolution runs, as evidenced by Figure 3.21.

As the central resolution is increased, medium-swirl cases exhibit slight changes in the character of far-field deposition at $5 \mathrm{~m} \mathrm{~s}^{-1}$ translation speed (cf. Figure 3.19a,b). At 25 $\mathrm{m} \mathrm{s}^{-1}$, wave modulations of far-field deposition occur to the right of the tornado. The physicality of these marks is called into question because their intensities vary strongly with grid resolution. Lateral scales of cycloidal deposition (when present) are roughly invariant to changes in the grid resolution at both swirl ratios explored in this sensitivity test.

Increasing the grid resolution by a factor of two over a larger area around the center of the simulation increases the required processing time from days to weeks, making it impractical for large sets of simulations. The present study focuses on the more robust marks (e.g. cycloids, trailing arcs, far-field swaths) rather than ones that appear resolution sensitive.

\subsubsection{Sensitivities to $\sigma$ and $d$ for constant $\sigma d$}

At high particle Reynolds number $R e_{p}$ (cf. Equation 2.12), the particle density and diameter, $\sigma$ and $d$, appear in the modeled equations only in the combination $\sigma d$, to a good approximation. Thus, results for "coarse sand" with $\sigma \sim 2000 \mathrm{~kg} \mathrm{~m}^{-3}$ and $d \sim 1 \mathrm{~mm}$ are also relevant to much larger debris of lower density (e.g. short lengths of dried cornstalk). This has been verified by varying $\sigma$ and $d$ independently while holding their product constant in different tornado regimes. Figure 3.22 shows sample damage tracks for medium- and high-swirl tornadoes from this experiment.

Variations in the extent of debris removal and the importance of far-field deposits is correlated with changes in debris terminal velocity, $w_{t}$ (cf. Equation 4.3) induced by changes in $d$. As $d$ is decreased, $R e_{p}$ also decreases and the drag coefficient $C_{D}$ (c.f. 2.11) becomes 

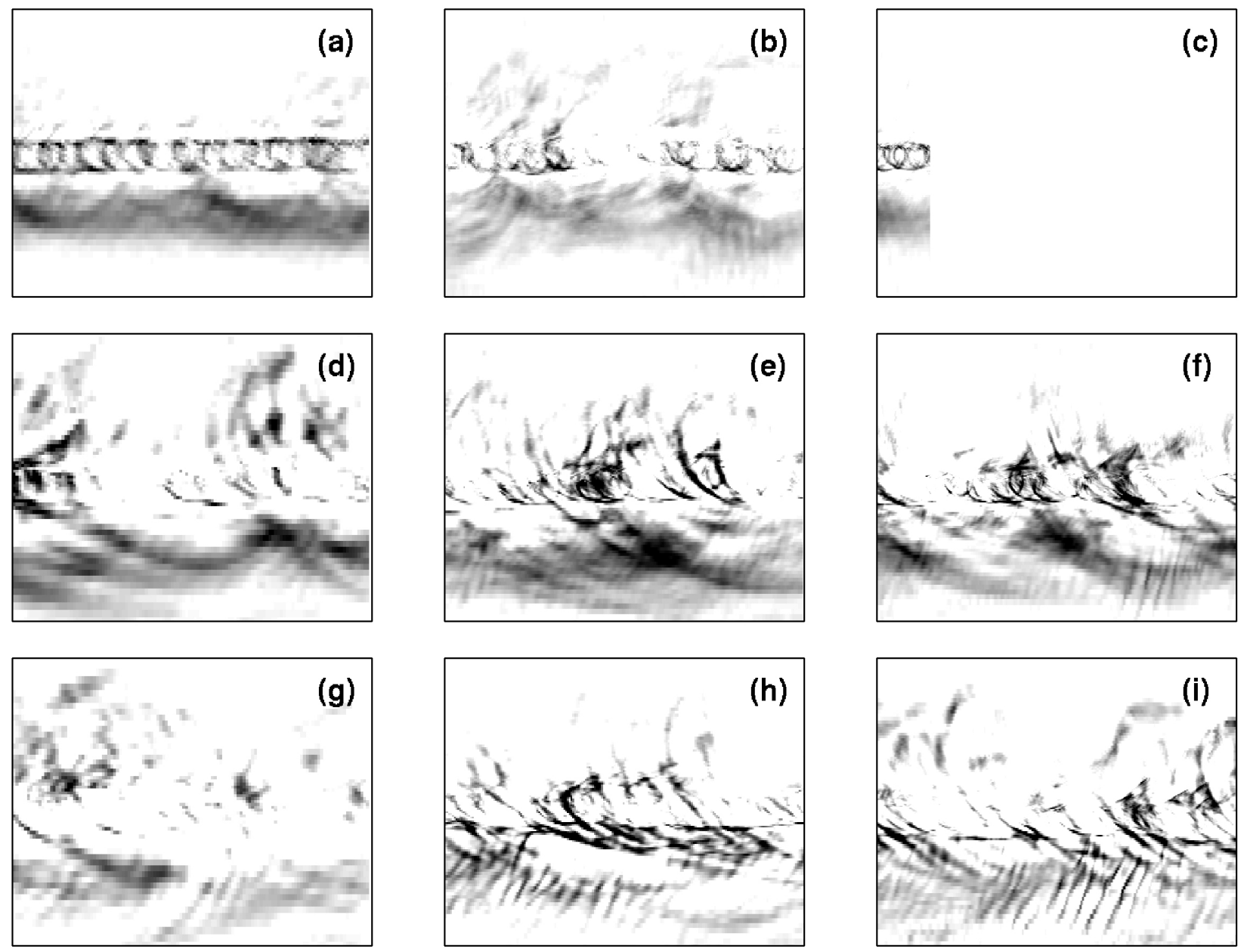

Figure 3.19: Net deposition patterns from simulated medium-swirl tornadoes with different central resolution. Translation velocities increase from top row to bottom row: $5 \mathrm{~m} / \mathrm{s}, 15$ $\mathrm{m} / \mathrm{s}$, and $25 \mathrm{~m} / \mathrm{s}$, respectively. Minimum central grid spacing decreases from left column to right column: $8 \mathrm{~m}, 5 \mathrm{~m}$, and $2 \mathrm{~m}$, respectively. Runs are: (a) dmg2033, (b) dmg2049, (c) dmg2049e, (d) dmg2026, (e) dmg2011, (f) dmg2011e, (g) dmg2032, (h) dmg2052, (i) dmg2052e. 

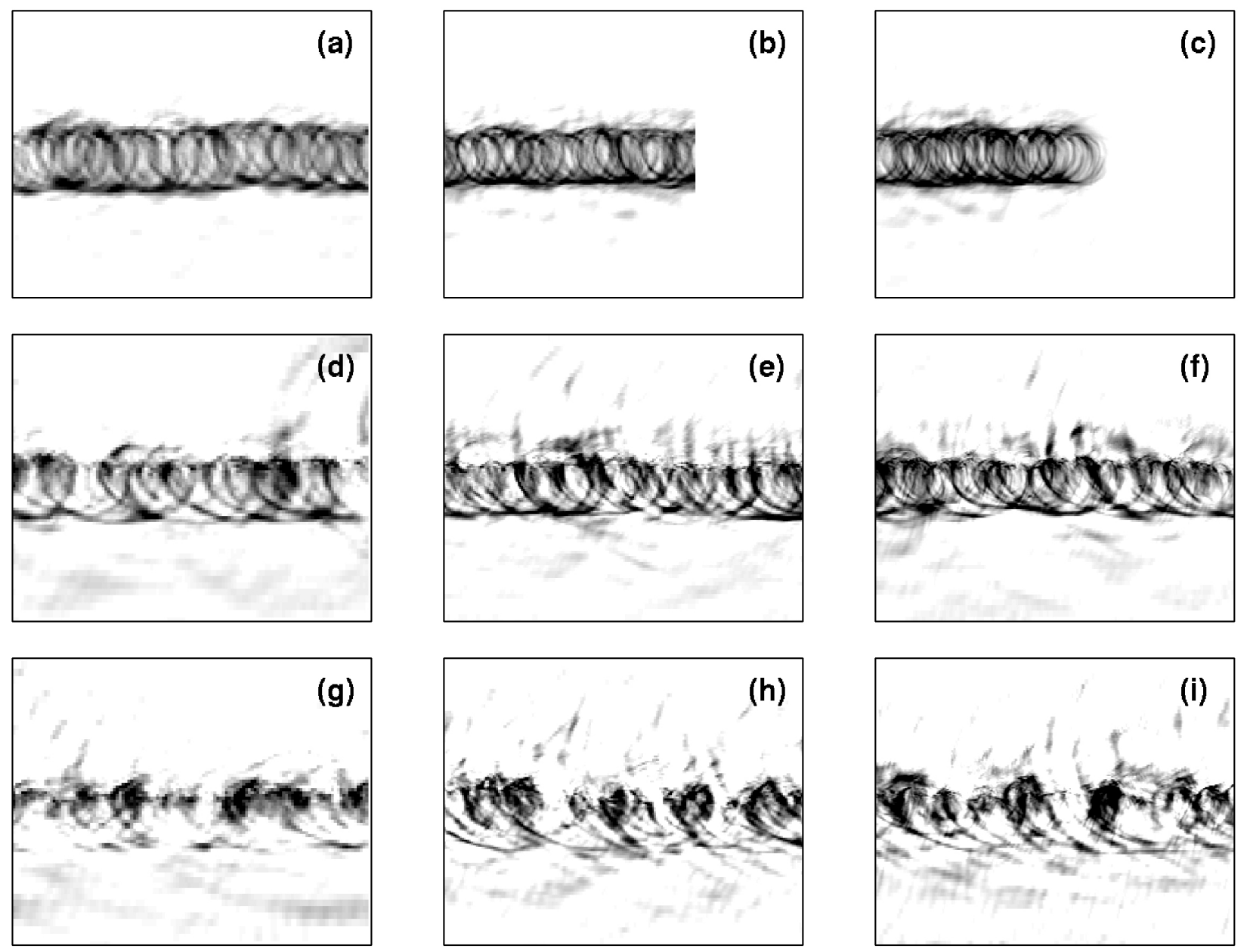

Figure 3.20: Net deposition patterns from simulated high-swirl tornadoes with different central resolution. Translation velocities increase from top row to bottom row: $5 \mathrm{~m} / \mathrm{s}, 15$ $\mathrm{m} / \mathrm{s}, 25 \mathrm{~m} / \mathrm{s}$. Minimum central grid spacing decreases from left column to right column: 8 m, 5 m, 2 m. Runs are: (a) dmg2035, (b) dmg2059, (c) dmg2059e, (d) dmg2028, (e) dmg2014, (f) dmg2014e, (g) dmg2034, (h) dmg2060, (i) dmg2060e. 

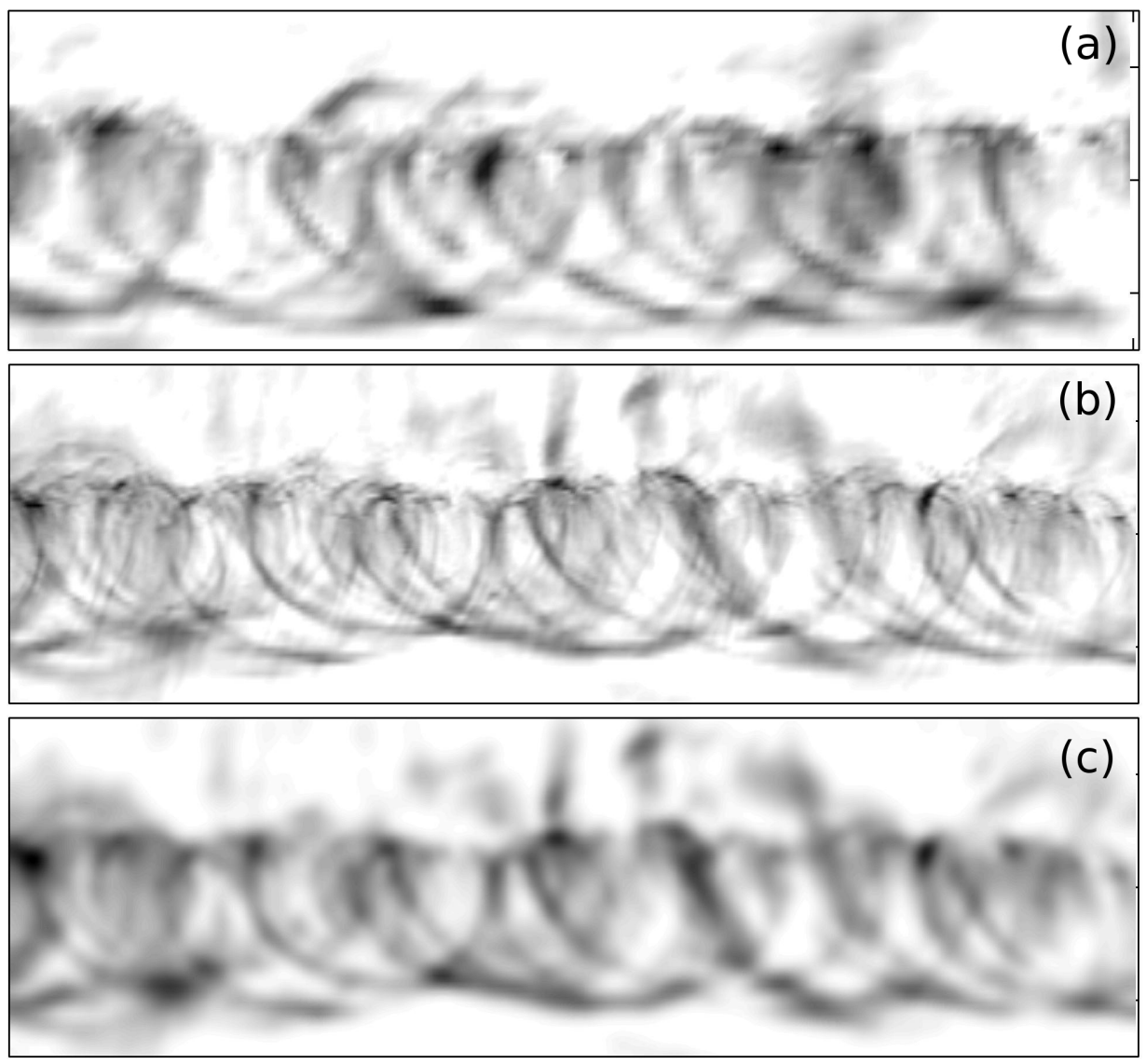

Figure 3.21: (a) Deposition track from Figure 3.20d; (b) deposition track from Figure 3.20f, showing finer-scale features than in (a); (c) deposition track from (b) after postprocessing with gaussian filter of width comparable to the grid resolution in (a), retaining only the strongest coarser-scale features. 
nonlinearly dependent on $R e_{p}$; in the low $R e_{p}$ regime, $w_{t}$ can be sensitive to changes in $d$, even if $\sigma$ is adjusted to hold the product $\sigma d$ constant. It must be noted that some choices of $\sigma$ and $d$ in Figure 3.22 are unphysical in that an unlimited surface source of large, lowdensity debris is unlikely in the field. However, this test demonstrates the relative numerical insensitivity of damage tracks to the balance between $\sigma$ and $d$, enabling comparisons between a wide range of $\sigma$ and $d$ without actually running the respective simulations.

\subsection{Multiple debris species}

Most simulations were performed with a monodisperse debris species available at the surface (i.e. all debris particles possessed the same $\sigma$ and $d$ ). However, in the field a distribution of debris can be expected. Simulations with multiple debris species were performed to gain insight into tornado structure in the presence of more-continuous distributions of debris. Figure 3.23 contains results from a subset of simulations which have three types of debris available at the surface (with respective $\sigma d=1 \mathrm{~kg} \mathrm{~m}^{-2}, 4 \mathrm{~kg} \mathrm{~m}^{-2}$, and $8 \mathrm{~kg} \mathrm{~m}^{-2}$ ).

In each multispecies run, the deposition patterns of individual constituent species qualitatively match one another to a large degree, which is largely a consequence of the coupling between individual species and the air flow. There is some variation in the subset of marks populated by each debris type; for example, lighter species (i.e., lower $\sigma d$ ) tend to produce relatively intense far-field deposition (cf. panels a and $\mathrm{j}$ of Figure 3.23) compared with the tracks of heavier species.

It is important to note that the total multispecies deposition tracks of Figure 3.23 qualitatively resemble individual single-species tracks in Figure 3.24. The surface debris pickup parameter $c_{a}$ for constituent species is important when drawing such comparisons; for example, $c_{a}=0.04$ for each constituent species in the multispecies track of Figure 3.23j, which qualitatively compares with that of the single species run with $c_{a}=0.12, \sigma d=3 \mathrm{~kg} \mathrm{~m}^{-2}$ in Figure 3.24g. Likewise, $c_{a}=0.12$ for each consitutent species in the multispecies track of 

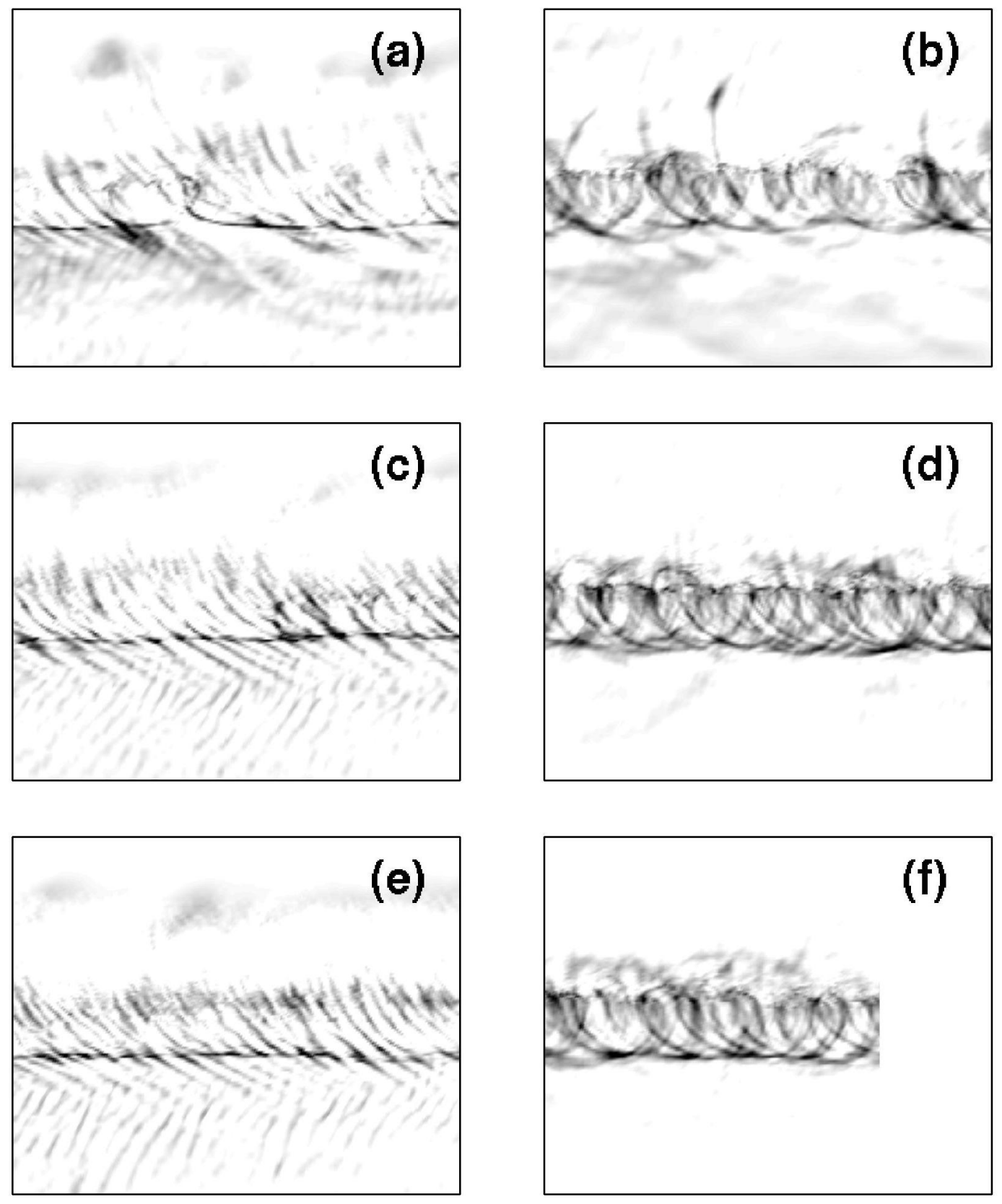

Figure 3.22: Surface tracks from (left column) medium-swirl and (right column) high-swirl cases with different $\sigma$ and $d$ while holding the product $\sigma d=2 \mathrm{~kg} \mathrm{~m}^{-2}$ constant. Variations in the tracks come from changes induced in $w_{t}$ in the low particle Reynolds number regime. (a,c,e) $U_{\text {trans }}=25 \mathrm{~m} \mathrm{~s}^{-1}, w_{t}=6.1,10.3,10.9 \mathrm{~m} \mathrm{~s}^{-1}$, respectively, with $\sigma=20000,500,100 \mathrm{~kg}$ $\mathrm{m}^{-3}$ and $d=0.2,8,40 \mathrm{~mm}$. (b,d,f) $U_{\text {trans }}=15 \mathrm{~m} \mathrm{~s}^{-1}, w_{t}=3.7,7.1,7.5 \mathrm{~m} \mathrm{~s}^{-1}$, respectively, with $\sigma=11330,250,100 \mathrm{~kg} \mathrm{~m}^{-3}$ and $d=0.177,8,20 \mathrm{~mm}$. Simulation runs are (a) dmg2288, (b) sig2015, (c) dmg2180, (d) dmg2181, (e) dmg2185, (f) sig1115. 
Figure $3.23 \mathrm{k}$, which is roughly comparable to the single species track with $c_{a}=0.36, \sigma d=8$ $\mathrm{kg} \mathrm{m}^{-2}$ in Figure 3.24l. In both multispecies cases, $c_{a}$ for the constituent species is one third that of the single-species cases, producing the same total surface debris removal at a given wind level.

However, it is not clear that ease of debris removal will be constant across the entire spectrum of available debris in the field. Figure 3.23k shows results from a multispecies test of the effects of nonuniformity in $c_{a}$ between constituent species. The debris loadings aloft in the respective single-species cases (e.g. the cases of Figures $3.24 \mathrm{a}, \mathrm{j}, \mathrm{k}$, with respective $\sigma d=1$ $\mathrm{kg} \mathrm{m} \mathrm{m}^{-2}, 4 \mathrm{~kg} \mathrm{~m}^{-2}$, and $8 \mathrm{~kg} \mathrm{~m}^{-2}$ ) give some indication of what the ease of lofting of each species might be when made available together at the surface. For example, lighter species are more readily collected and lofted by the tornado as a whole, so in the presence of a given local wind level lighter species will likely constitute a greater debris flux than heavier species.

As such, the values of $c_{a}$ for the individual species of Figure 3.23c,f,i were scaled by the debris loadings aloft in their respective single-species runs. The lightest species (e.g. Figure $3.23 \mathrm{c}$ ), with $\sigma d=1 \mathrm{~kg} \mathrm{~m}^{-2}$, dominated the qualitative character of the total deposition track due to its significant contribution to the total surface debris flux. As in the other multispecies cases, the total track in this case qualitatively matched a given single-species track (e.g. Figure 3.24c, with $c_{a}=0.12, \sigma d=1.5 \mathrm{~kg} \mathrm{~m}^{-2}$ ).

These observations potentially simplify comparisons between single-species simulation results and real surface marks created in the presence of a continuous distribution of debris. It is plausible that surface marks in the presence of a debris continuum will be similar to that of a monodisperse debris population with an "effective" $\sigma d$ and $c_{a}$. All parts of the debris continuum will likely contribute to some subset of the most prominent marks, rather than individual parts of the spectrum creating vastly different patterns on the ground. 

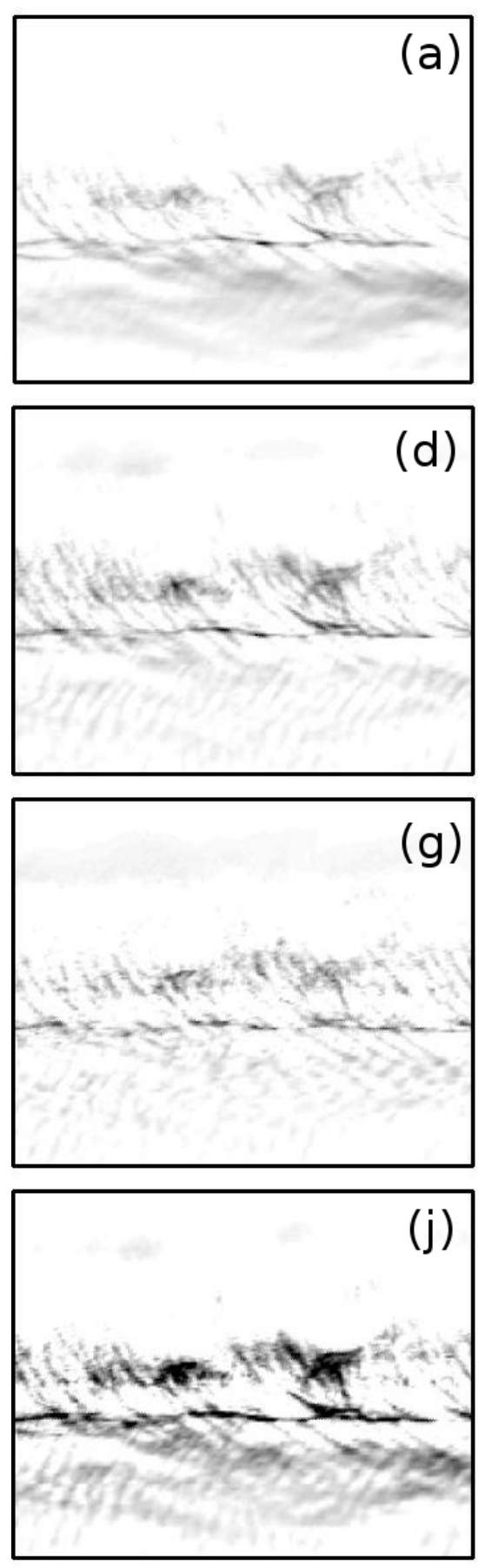
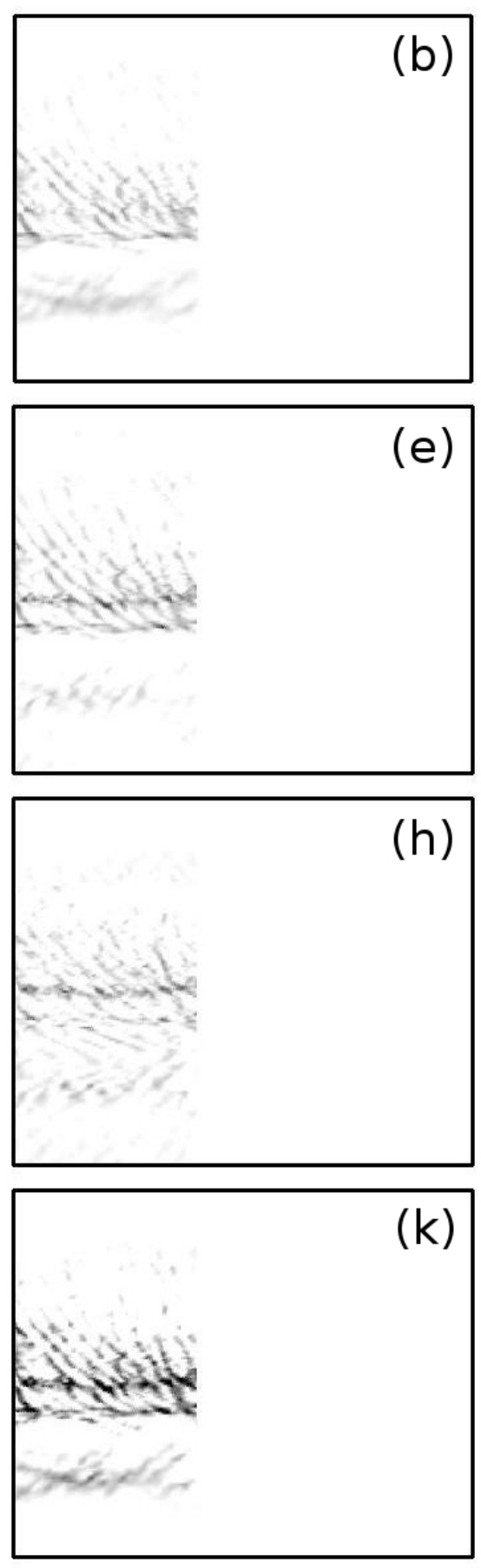

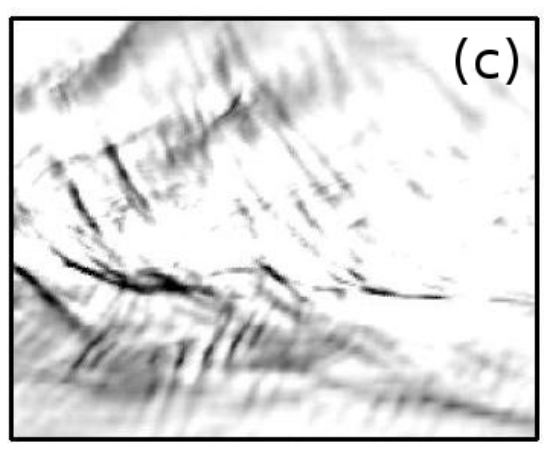

(f)

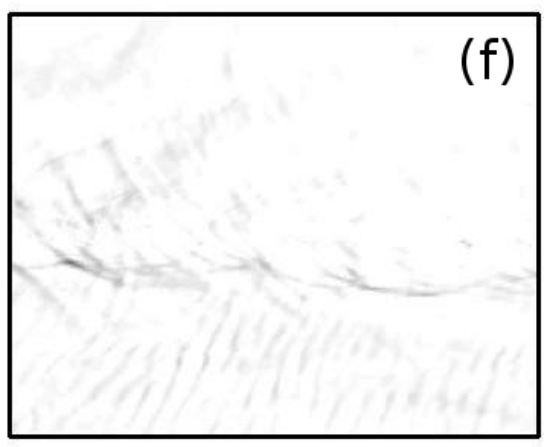

(i)

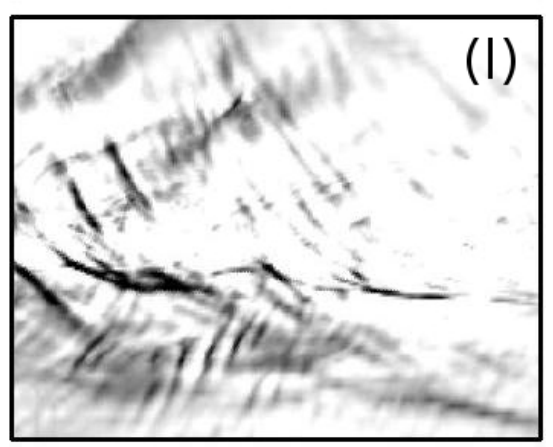

Figure 3.23: Deposition tracks for multispecies runs with different subgrid turbulence debris pickup parameter $c_{a}$. Each simulated tornado is moving to the right at $25 \mathrm{~m} / \mathrm{s}$ and has three species: (top row) $\sigma d=1 \mathrm{~kg} \mathrm{~m}^{-2}$, (second row) $\sigma d=4 \mathrm{~kg} \mathrm{~m}^{-2}$, (third row) $\sigma d=8$ $\mathrm{kg} \mathrm{m}^{-2}$, (bottom row) total deposition of all three species. Surface parameterizations: (left column) $c_{a}=0.04$ (run dmg2249, grayscale clipped to $15 \mathrm{~kg} \mathrm{~m}^{-2}$ ), (middle column) $c_{a}=0.12$ (run 3sp3026, grayscale clipped to $45 \mathrm{~kg} \mathrm{~m}^{-2}$ ), (right column) $c_{a}$ varies per debris species: $c_{a}=0.0950,0.0175,0.0070$ from highest to lowest $A_{v}$ (run dmg2267, grayscale clipped to 15 $\left.\mathrm{kg} \mathrm{m}^{-2}\right)$. 

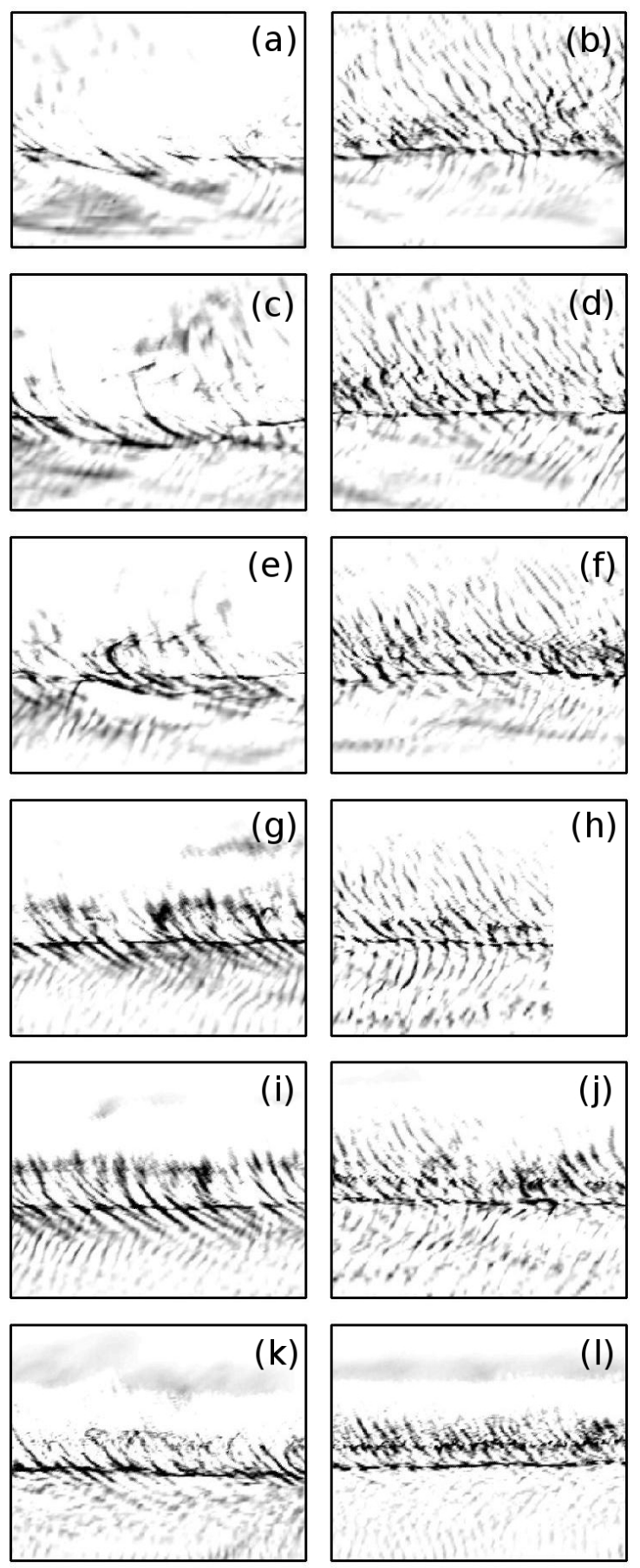

Figure 3.24: Deposition tracks from a medium-swirl collection of single-species runs with $U_{\text {trans }}=25 \mathrm{~m} \mathrm{~s}^{-1}$ and different subgrid turbulence debris pickup parameter $c_{a}$. Left to right: $c_{a}=0.12,0.36$. Top to bottom: $\sigma d=1.0,1.5,2.0,3.0,4.0,8.0$. The darkest shade of gray in the lefthand column corresponds to a deposition level of $15 \mathrm{~kg} \mathrm{~m}^{-2}$, and the intensity levels of the right column were scaled by a factor of 3 relative to the left column to provide uniform intensity across the rows. Simulation runs are (a) dmg3033, (b) dmg2246, (c) dmg2245, (d) dmg2252, (e) dmg2052, (f) dmg2270, (g) dmg2244, (h) dmg2251, (i) dmg3026, (j) dmg2247, (k) dmg3023, (l) dmg2248. 


\section{Chapter 4}

\section{Simulation parameters varied}

The effects of many physical variables must be considered in order to understand tornado and debris cloud structures and surface marks that result from a given configuration. In this chapter, the most important quantities are identified in Section 4.1, and a handful of dimensionless combinations (developed previously by Lewellen et al. (2008) and references therein) are introduced in Section 4.2 to motivate the choice of simulation runs in the present study (e.g. as listed in the Appendix).

\subsection{Parameters involved}

Lewellen et al. (2000) showed that the most important factors governing tornado corner flow structure in the absence of debris are the levels of near-surface swirl and horizontal convergence combined with properties of the vortex aloft. In the large-eddy simulations, the former are governed primarily by the lateral boundary conditions near the surface and the effects of surface friction on the low-level flow. Surface friction is parameterized by the surface roughness length $z_{0}$, and its effects encapsulated to lowest order in the depleted angular momentum flux through the vortex, $\Upsilon$, as are the levels of near-surface convergence and angular momentum. Vortex properties aloft are affected by the top boundary conditions and the angular momentum outside the vortex core; these elements are captured by the far- 
field angular momentum $\Gamma_{\infty}$ and the maximum swirl velocity aloft $V_{c}$, which combine to give a characteristic radial vortex scale $R_{c}$. At high Reynolds number, viscous stresses drop out of the fluid equations; the air viscosity, $\mu \approx 1.78 \times 10^{-5} \mathrm{~kg} \mathrm{~m}^{-1} \mathrm{~s}^{-1}$, is low enough that essentially all of the flow away from the surface are typically well within the high- $R_{e}$ limit, given length and velocity scales of $\sim 10 \mathrm{~m}$ and $\sim 10 \mathrm{~m} \mathrm{~s}^{-1}$ for the most important turbulent eddies. When debris is not present, air density $\rho$ is the sole mass scale in the simulations.

Lewellen et al. (2008) showed that in the presence of debris the combination $\sigma d$ becomes important. Gravitational accleration, $\sigma g$, cannot be neglected when debris is present. Surface-relative translation $U_{\text {trans }}$ introduces flow asymmetries in the surface layer and tilts the central vortex (Lewellen et al., 1997), sometimes significantly affecting the visual appearances and relative weightings of different classes of surface marks (Lewellen and Zimmerman, 2008). Surface parameters set in the LES code (c.f. Equations 2.14-2.16 and 2.18-2.19) can affect the overall amount and spatial extent of debris pickup and redeposition.

Thus, even at the lowest order of approximation, there are at least seven primary variables affecting debris-laden tornado structure, and ultimately the surface marks left behind: $V_{c}$, $\Gamma_{\infty}, \Upsilon, \rho, g, \sigma d$, and $U_{\text {trans }}$. In the simulations, $g, \sigma d, U_{\text {trans }}$, and $\Gamma_{\infty}$ were specified directly, $V_{c}$ and $\Upsilon$ were set indirectly through the lateral and top boundary flow conditions, and $\rho$ was not changed. The effects of the surface roughness length $z_{0}$ on tornado structure are largely captured by $\Upsilon$ and were shown to be of at most secondary importance by Lewellen et al. (2000), as are other surface properties.

\subsection{Dimensional analysis}

Varying all of the physical parameters would require an unweildy number of simulations but dimensional analysis reduces the set to at least four governing parameters that simplify the necessary set of runs, which are outlined here. The principle simulation set outlined in

the Appendix was chosen to explore the effects of independent variations in the dimensionless 
parameters on properties of surface tracks.

When debris is not present, the corner flow swirl ratio $S_{c}=R_{c} \Gamma_{\infty} / \Upsilon$ is the important dimensionless parameter, with basic length, velocity, and mass scales given by $R_{c}, V_{c}$, and $\rho$ (Lewellen et al., 2000). Two other dimensionless parameters become important in the presence of debris (Lewellen et al., 2008):

$$
\begin{gathered}
A_{v}=V_{c} / w_{t} \\
A_{a}=V_{c}^{2} /\left(g R_{c}\right)
\end{gathered}
$$

where $w_{t}$ is the debris terminal velocity in freefall:

$$
w_{t}=\sqrt{\frac{4 g d \sigma}{3 C_{D} \rho}}
$$

The expressions for $A_{v}$ and $A_{a}$ were chosen for ease of interpretation: the velocity ratio $A_{v}$ parameterizes the maximum amount of debris slip possible with respect to the air flow (increasing $A_{v}$ lessens the maximum potential amount of slip) and the acceleration ratio $A_{a}$ measures the importance of a characteristic radial acceleration relative to gravity. In this study, tornado translation critically affects the near-surface flow responsible for generating surface marks. The translation speed $U_{\text {trans }}$ is non-dimensionalized in the combination

$$
A_{t}=U_{\text {trans }} / V_{c}
$$

which captures the importance of the translation velocity with respect to the swirl velocity scale aloft. Placing $U_{\text {trans }}$ in the numerator avoids singularities when $U_{\text {trans }} \simeq 0$.

The ranges of parameters explored were $0<S_{c}<13,1.5<A_{v}<30,0.5<A_{a}<77$, $0.05<A_{t}<0.6$, enabled through changes in the constituent physical variables. For instance, $S_{c}$ was typically varied by changing $\Upsilon, \Gamma_{\infty}$, or $R_{c}$ by modifying the boundary flow conditions. $A_{a}$ was tailored by changing $g$ with respect to $V_{c}^{2} / R_{c}$, and $A_{v}$ was modified by changing 
$\sigma d / \rho$ to produce a desired value of $w_{t}$ in the presence of gravitational acceleration $g . A_{t}$ was selected independently by modifying $U_{\text {trans }}$. The parameters $A_{a}, A_{v}$, and $A_{t}$ were also affected by rescaling the entire flow velocity field, thus effectively changing $V_{c}$ while holding $S_{c}$ constant.

Most of the dimensional parameters involved (and also $S_{c}$ ) are measured in simulations that have no debris, avoiding uncertainties brought about by interaction of debris with the air flow (c.f. Section 1.2).

\subsection{Bounding values on physical and dimensionless pa- rameters}

Alexander and Wurman (2008) recently compiled an updated climatology of supercell tornadoes based on a collection of several thousand radar scans through approximately 150 individual tornadoes between 1995-2008. Their data helps to constrain the values of dimensionless parameters used in the simulations and expected in the field by placing bounds on the constituent physical parameters. In particular, they provided a representative distribution of peak velocity differences across the upper core, enabing estimation of both $V_{C}$ and $R_{C}$ across a wide range of tornadoes $\left(R_{C}\right.$ is taken as half the distance between the peak incoming and outgoing velocities from the point of view of the Doppler radar source). Doppler slices with a peak velocity difference less than $40 \mathrm{~m} \mathrm{~s}^{-1}$ and core diameter greater than 2000 $\mathrm{m}$ were removed from the dataset, and the inferred values of $V_{C}$ ranged from about $20 \mathrm{~m}$ $\mathrm{s}^{-1}$ to $100 \mathrm{~m} \mathrm{~s}^{-1}$, and $R_{C}$ ranged from less than $50 \mathrm{~m}$ to $1000 \mathrm{~m}$. Although the lowest $\Gamma_{\infty}$ derivable from these values is approximately $1000 \mathrm{~kg} \mathrm{~m}^{2} \mathrm{~s}^{-1}$, to achieve winds of at least EF1 rating on the Enhanced Fujita scale would require $V_{C} \sim 40 \mathrm{~m} \mathrm{~s}^{-1}$. A distribution of vortex translation velocities observed in the field was also provided in (Alexander and Wurman, 2008), suggesting that a reasonable upper bound on $U_{\text {trans }}$ is about $25 \mathrm{~m} \mathrm{~s}^{-1}$ (with a median value of about $13 \mathrm{~m} \mathrm{~s}^{-1}$ ); simulated translation speeds were within $U_{\text {trans }} \leq 25 \mathrm{~m} \mathrm{~s}^{-1}$, except 
for a few select cases. The lower bound $V_{C}=40 \mathrm{~m} \mathrm{~s}^{-1}$ and upper bound $U_{\text {trans }}=25 \mathrm{~m}$ $\mathrm{s}^{-1}$ give an upper bound on $A_{t}$ of about $A_{t}^{\max } \sim 0.625$. In the LES simulations, $A_{t}$ rarely exceeded 0.55 .

The lowest $A_{a}$ would occur for a tornado with $V_{C}$ around its lowest possible value and $R_{C}$ at its highest possible value. Using the data from (Alexander and Wurman, 2008) with gravity set at $g=9.81 \mathrm{~m} \mathrm{~s}^{-1}$, the minimum possible $A_{a}$ is $A_{a}^{\min } \sim 0.16$ for tornadoes mapped to EF1 or greater on the Enhanced Fujita Scale. In the opposite extreme, where $V_{C}$ is at its highest while $R_{C}$ is at its lowest, the maximum possible $A_{a}$ is $A_{a}^{\max } \sim 20.4$. Taking the limit where $R_{C}$ goes to zero, $V_{C}$ must also diminish to zero, so $A_{a}^{\max }$ on the order of 20 is probably a reasonable upper bound. For the majority of the simulations, $A_{a}$ ranged from about 0.05 to 8.00 ; the regime $A_{a}>20$ was attained in a small handful of cases by decreasing $g$ by a factor of three or more.

Sand-like debris is used in most of the simulations, with terminal speed $w_{t}$ typically within $3-13 \mathrm{~m} \mathrm{~s}^{-1}$ and $A_{v}$ within about 5-30. These numbers are consistent with debris ranging from small raindrops to small hailstones immersed in tornadoes (Dowell et al., 2005) with $V_{C}$ and $R_{C}$ within the ranges measured in (Alexander and Wurman, 2008). Sensitivity checks showed that damage tracks and debris aerodynamics for different simulations were largely insensitive to changes in $\sigma$ and $d$ as long as the product $\sigma d$ was left unchanged (c.f. Section 3.4.3); thus, simulations of sand are relevant to much larger debris of much lower density (e.g. corn stubble, which has been documented in the literature). 


\section{Chapter 5}

\section{Taxonomy and detailed physical origins of marks}

Features such as "cycloidal" and "drift" marks, "lineation" and "scalloping", commonly identified in surface tracks of real tornadoes by T. T. Fujita and his colleagues 30-40 years ago (Agee et al., 1975, 1977; Davies-Jones et al., 1978; Fujita, 1966, 1967, 1981; Fujita et al., 1967, 1970, 1976a,b) are often prominent in the simulated tracks. Historically, the appearance of well-defined lines of deposition were almost invariably interpreted as the results of the central convergence of a vortex or vortices traveling along the surface, either the main tornado vortex (producing "lineal" marks) or secondary "suction" vortices rotating about the tornado (producing cycloidal marks or scalloping). Figure 5.1 summarizes Fujita et al.'s interpretations of such marks. The emphasis on paths of individual vortices was found in the present work to be unjustified: simulated secondary vortices were generally too small to gather a significant amount of debris, and all of the types of marks previously attributed to secondary vortices were observed even in some simulations with only a single, main vortex near the ground.

A more general set of flow features was found to be responsible for the strongest marks. Competition between the central vortex, regions of alternating convergence and divergence 
(e.g. surface rolls) in the near-surface inflow, and far-field downdrafts of fully-lofted debris give rise to a convolution of deposition and removal events in space and time. All of the marks in the historical taxonomy (e.g. Figure 5.1) have been encountered, with their relative intensities and geometric properties varying through effects of the dimensionless parameters on the physical mechanisms responsible. Also, distinct categories of marks have been found to arise under time-varying boundary conditions; they closely resemble certain marks from quasisteady simulations. Debris deposition is the main focus of this chapter for two reasons: removal signatures are generally more diffuse and the shapes of simulated deposition marks correlate well with those of surface marks observed in the field.

In the following sections, deposition marks from a large collection of quasisteady simulations are grouped into qualitative classes and their physical origins are reinterpreted in terms of the dimensionless parameters describing the tornado system. Deposition tracks and sample surface fluxes from a primary subset of runs are shown in Figures 5.3-5.10 and are referred to throughout the Chapter. In the damage tracks of Figures 5.3-5.10, grayscale levels have been scaled by $A_{t}$ to provide uniformity in intensity. Though instantaneous local debris fluxes are generally of comparable magnitudes in each given simulation subset, the total time-integrated deposition is directly proportional to the time spent over each surface point (i.e., it is inversely proportional to $\left.U_{\text {trans }}\right)$. Additional scaling by $\left(\rho R_{c}\right)^{-1}$ nondimensionalized the net deposition signatures.

\subsection{Deposition underneath the central vortex}

In the model of Fujita and colleagues, some fraction of debris follows the sharp corner flow upturn to be ejected aloft and the rest is deposited underneath the vortex (e.g. Figure 5.2). Radial and vertical debris slip are critical components of this interpretation; without either (i.e. in the high- $A_{v}$ limit), debris would simply follow the corner flow aloft, never to be deposited underneath the vortex. However, simulation results showed that the central 


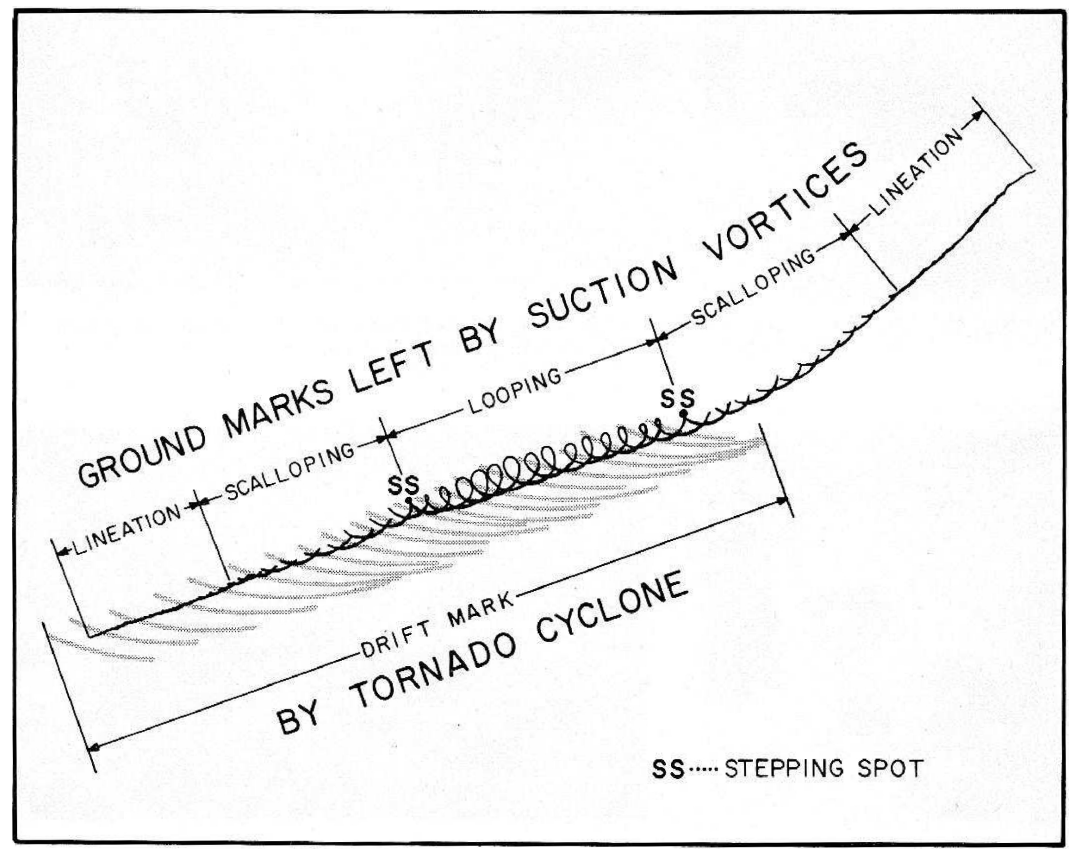

Figure 5.1: Fujita et al.'s taxonomy of marks emphasizing secondary vortices as the primary physical process behind deposition marks, from Fujita (1976).

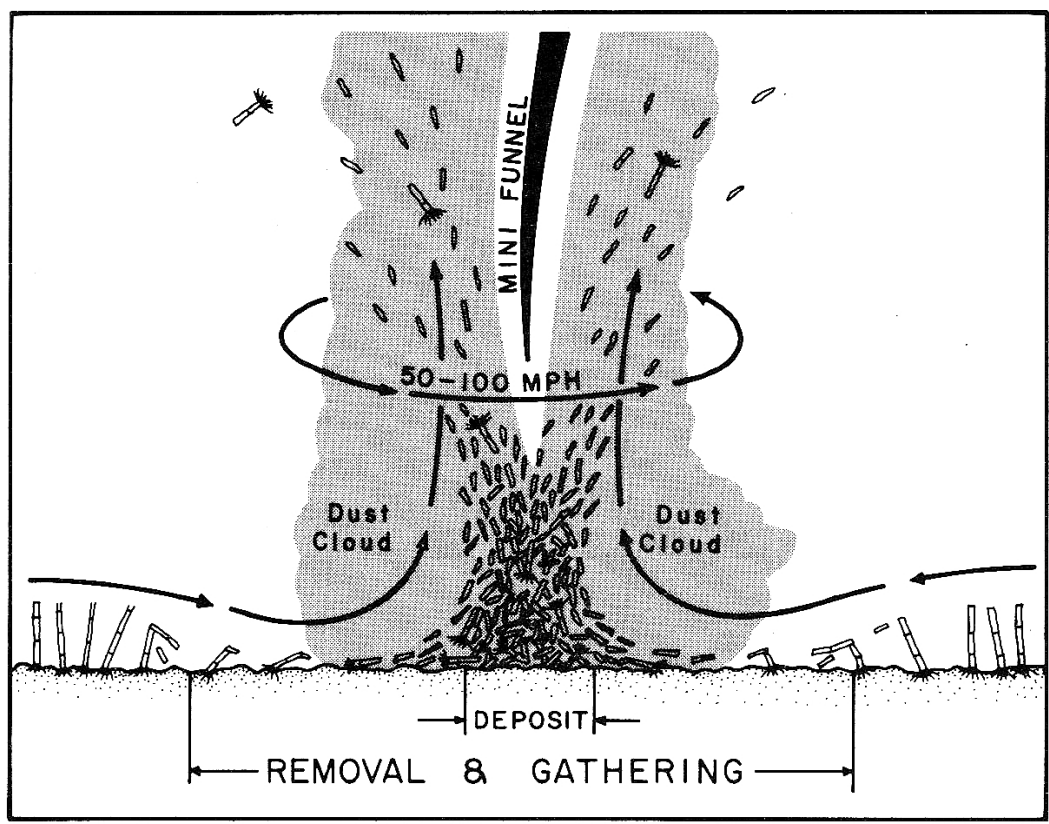

Figure 5.2: Fujita et al.'s model of a single vortex collecting debris into a spot at the ground. Some debris is able to follow the corner flow and is ejected aloft. From Fujita (1976). 

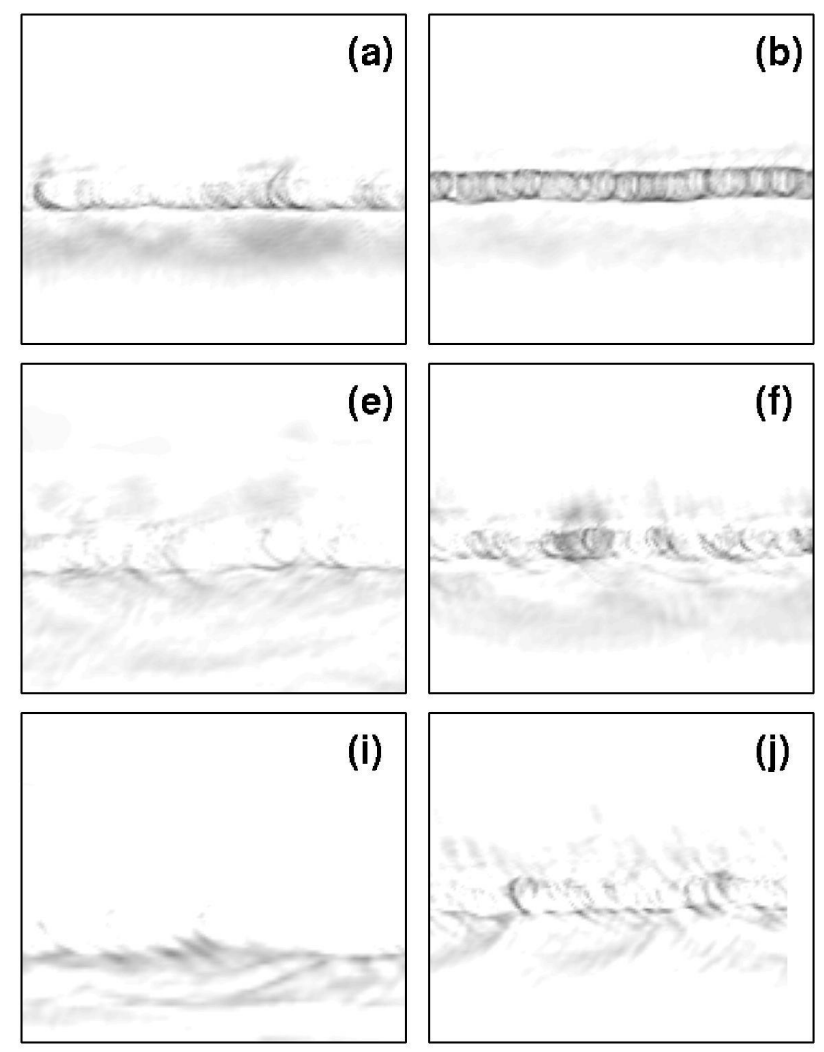
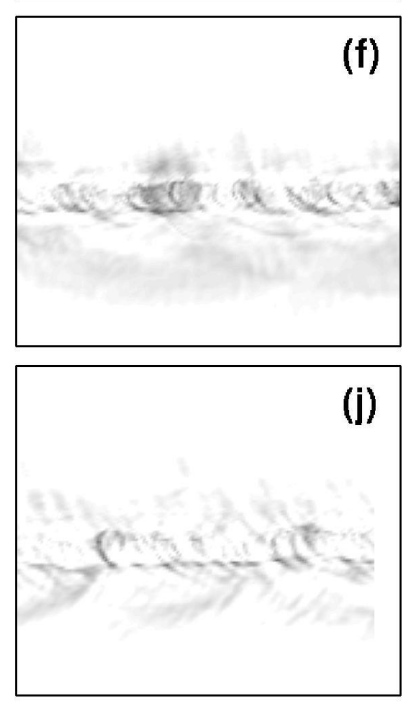
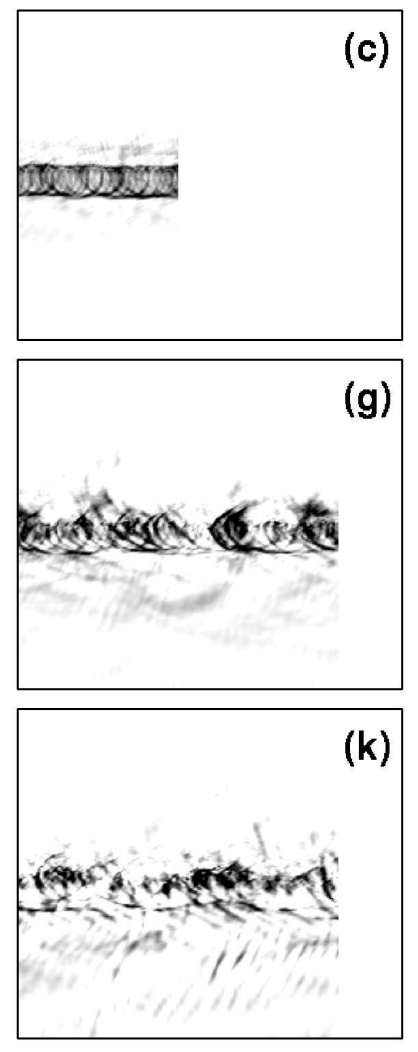
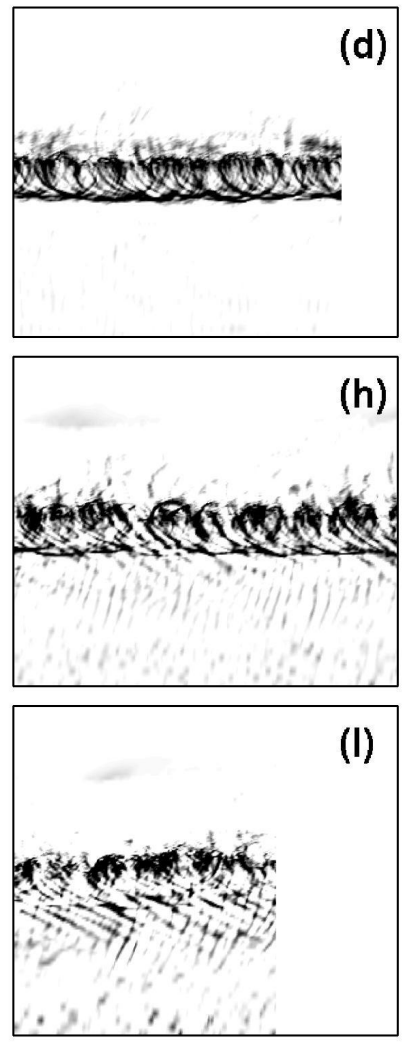

Figure 5.3: Deposition tracks for a collection of tornadoes with, primarily varying $S_{c}$ (let to right) and $A_{t}$ (top to bottom). Left to right: $A_{v}=8.3,10.3,9.95,8.2, A_{a}=$ $2.3,1.9,1.9,1.7, S_{c}=1.9,3.0,5.6,12.4$; (top row) $A_{t}=0.11,0.06,0.08,0.21$, (middle row) $A_{t}=0.32,0.17,0.26,0.36$, (bottom row) $A_{t}=0.53,0.33,0.44,0.50$. Lateral dimensions span $\pm 3 R_{c}$. Grayscale intensities have been scaled by $A_{t}\left(\rho R_{c}\right)^{-1}$; the darkest shades of gray correspond to a dimensionless deposition level of $1.25 \times 10^{3}$. Simulation runs are (a) dmg3321, (b) dmg2192, (c) dmg3242, (d) dmg2081, (e) dmg3322, (f) dmg2193, (g) dmg3243, (h) dmg2082, (i) dmg3323, (j) dmg2203, (k) dmg3244, (l) dmg2084. 

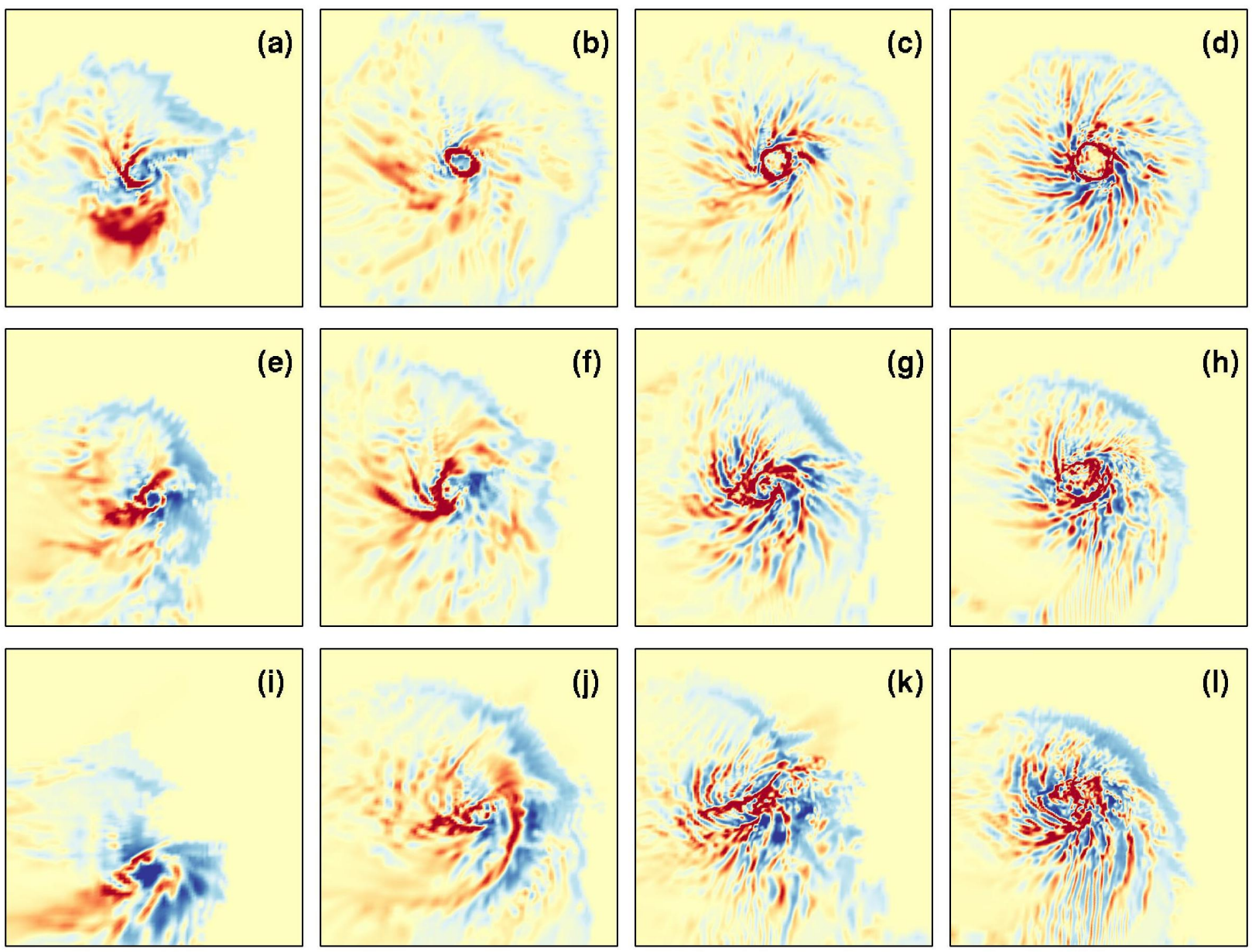

Figure 5.4: Sample instantaneous vertical debris flux at the surface for the cases in Figure 5.3. Fluxes have been normalized by $V_{c}$; the quantity plotted is $w_{d} D / V_{c}$, where $w_{d}$ is the absolute debris velocity and $D=\rho_{d} / \rho$ is the dimensionless debris mass loading. Spatial dimensions have been normalized to $R_{c}$ and span $\pm 3 R_{c}$. Deep red/blue correspond to normalized flux levels of magnitude 0.025. To capture the central flow, certain panels have been shifted forward by the following amounts: (e) $R_{c}$, (i) $2 R_{c}$, (j) $R_{c}$. 

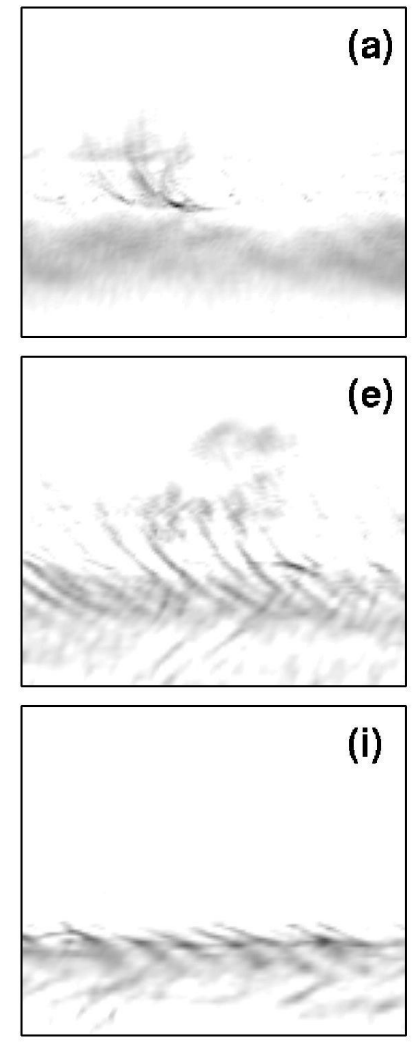
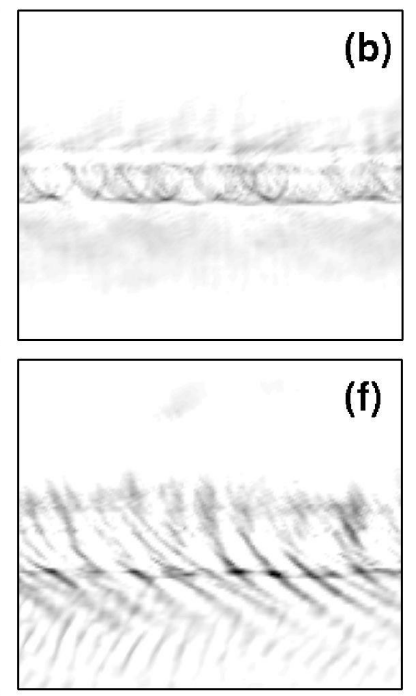

(i)

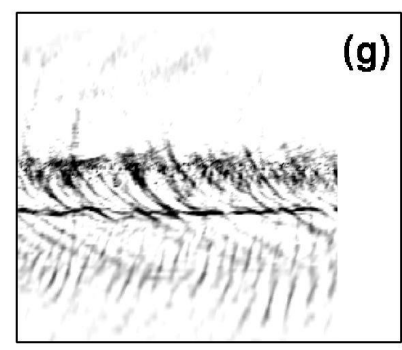

(k)

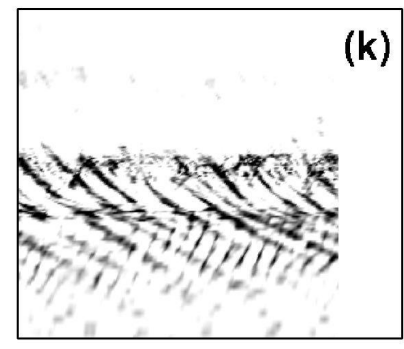

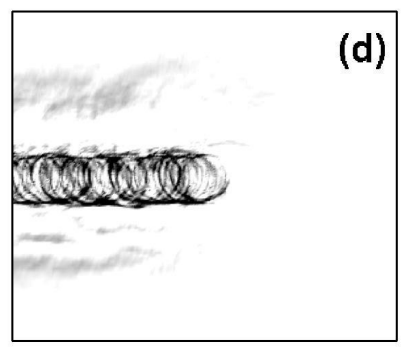

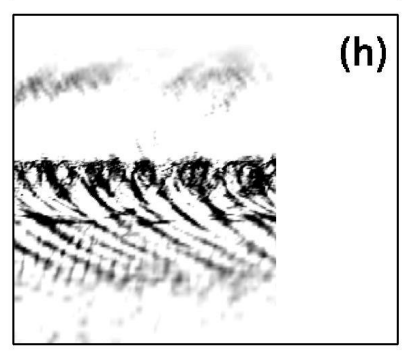

(h)

Figure 5.5: Deposition tracks for a collection of tornadoes with $A_{a} \sim 8$ and $A_{v} \sim 11$, primarily varying $S_{c}$ (left to right) and $A_{t}$ (top to bottom). Left to right: $A_{v}=10.4,10.5,10.1,12.3$, $A_{a}=8.0,7.7,7.8,7.9, S_{c}=1.9,3.0,5.6,12.4$; (top row) $A_{t}=0.11,0.06, \mathrm{n} / \mathrm{a}, 0.07$, (middle row) $A_{t}=0.32,0.28,0.26,0.36$, (bottom row) $A_{t}=0.53, \mathrm{n} / \mathrm{a}, 0.44, \mathrm{n} / \mathrm{a}$. Lateral dimensions span $\pm 3 R_{c}$. Grayscale intensities have been scaled as in Figure 5.3; the darkest shades of gray correspond to a dimensionless deposition level of $1.25 \times 10^{3}$. Simulation runs are (a) dmg2218, (b) dmg3024, (d) dmg2212, (e) dmg2219, (f) dmg3026, (g) dmg2226, (h) dmg2195, (i) dmg2220, (k) dmg2227. 

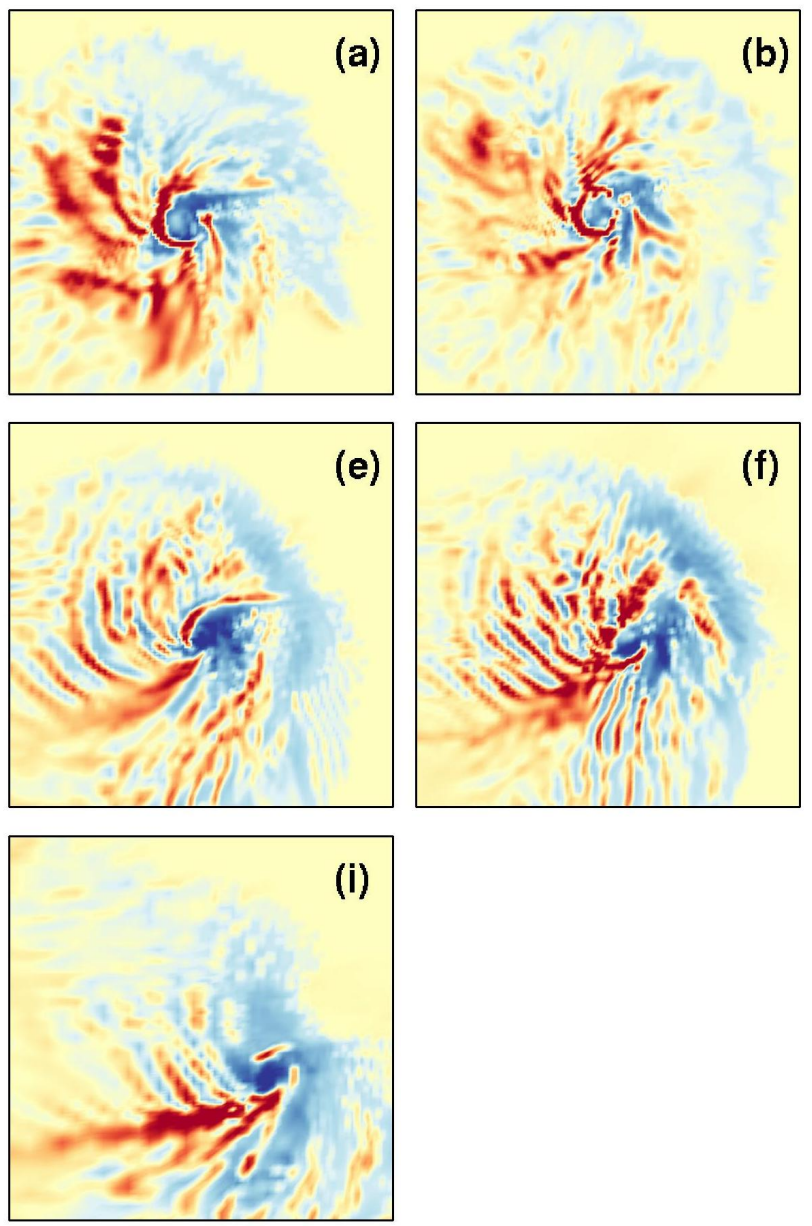
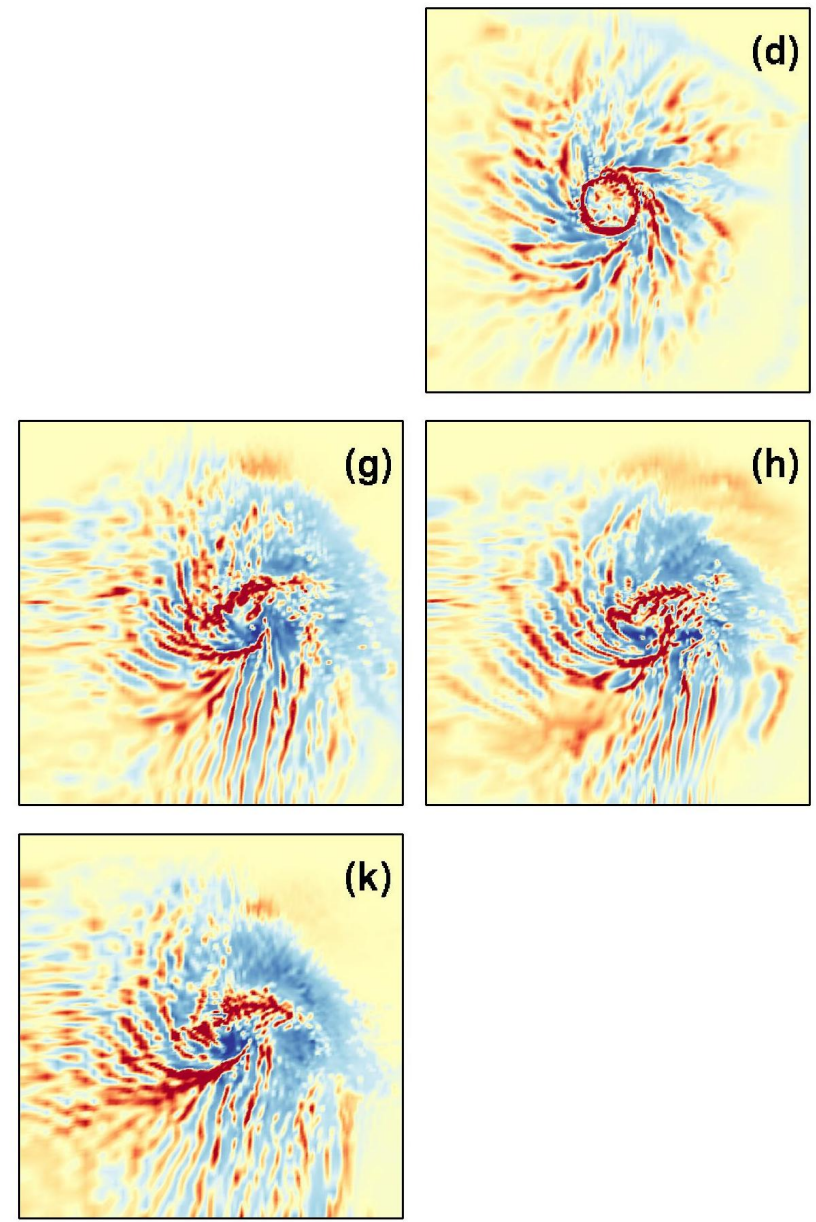

Figure 5.6: As in Figure 5.4 except for the deposition tracks in Figure 5.5. Panels (e,f,g,h) are shifted forward by $R_{c}$, and panel (i) was shifted forward by $3 R_{c}$. 

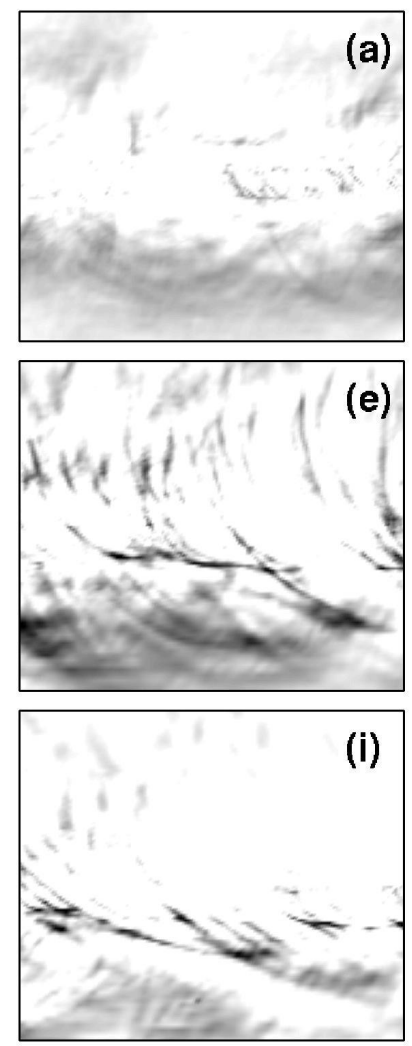
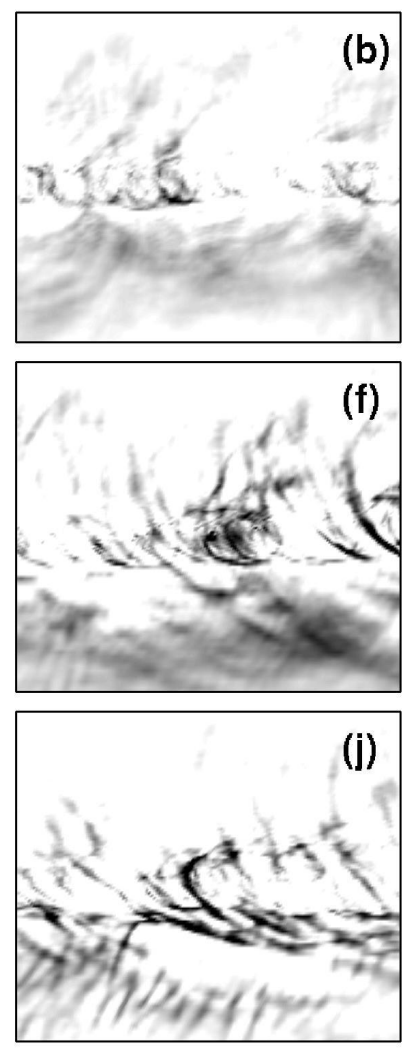
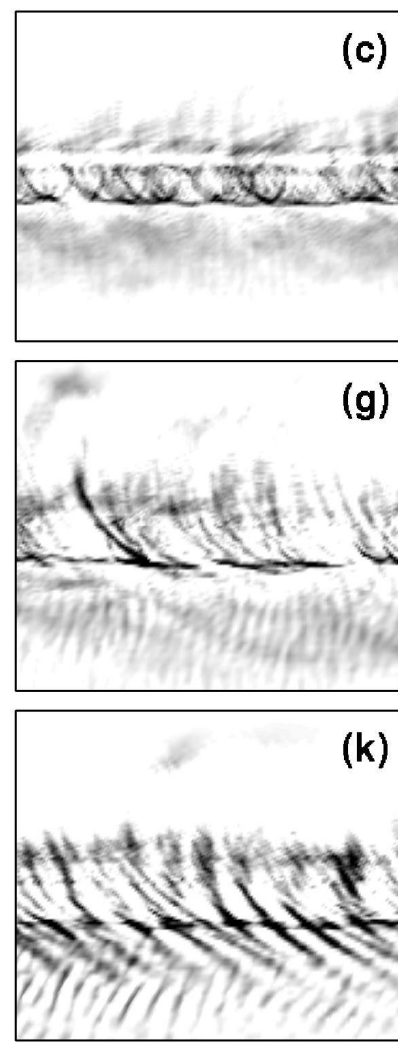
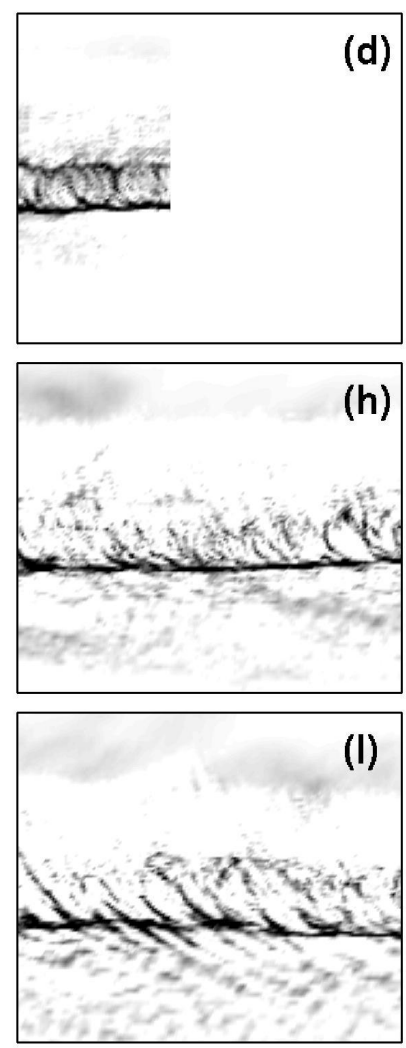

Figure 5.7: Deposition tracks for a collection of tornadoes with $S_{c}=3.0$ and $A_{a}=7.7$, primarily varying $A_{v}$ (left to right) and $A_{t}$ (top to bottom). Left to right: $A_{v}=$ 23.8, 15.9, 10.6, 7.2. Top to bottom: $A_{t}=0.06,0.17,0.28$. Lateral dimensions span $\pm 3 R_{c}$. Grayscale intensities have been scaled as in Figure 5.3; the darkest shades of gray correspond to a dimensionless deposition level of $0.5 \times 10^{3}$. Simulation runs are (a) dmg3031, (b) dmg2049, (c) dmg3024, (d) dmg3021, (e) dmg3032, (f) dmg2011, (g) dmg3025, (h) dmg3022, (i) dmg3033, (j) dmg2052, (k) dmg3026, (l) dmg3023. 

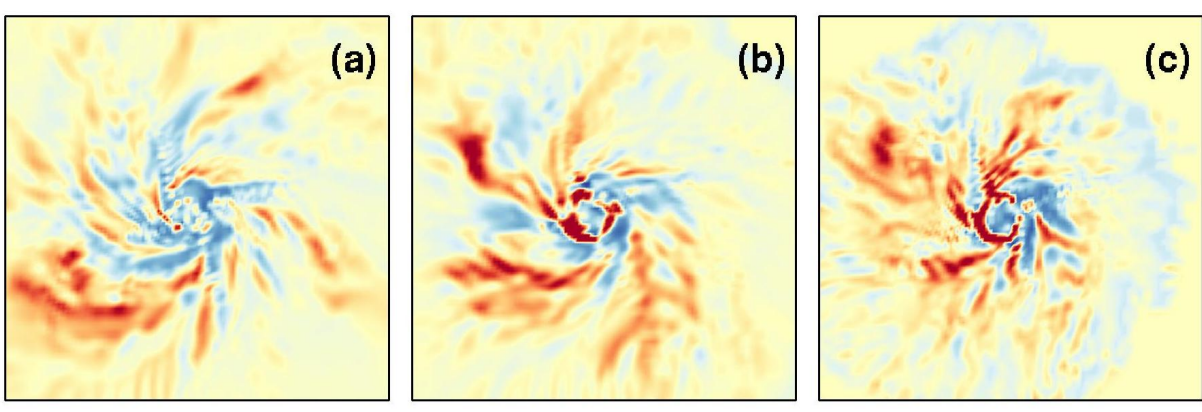

(c)
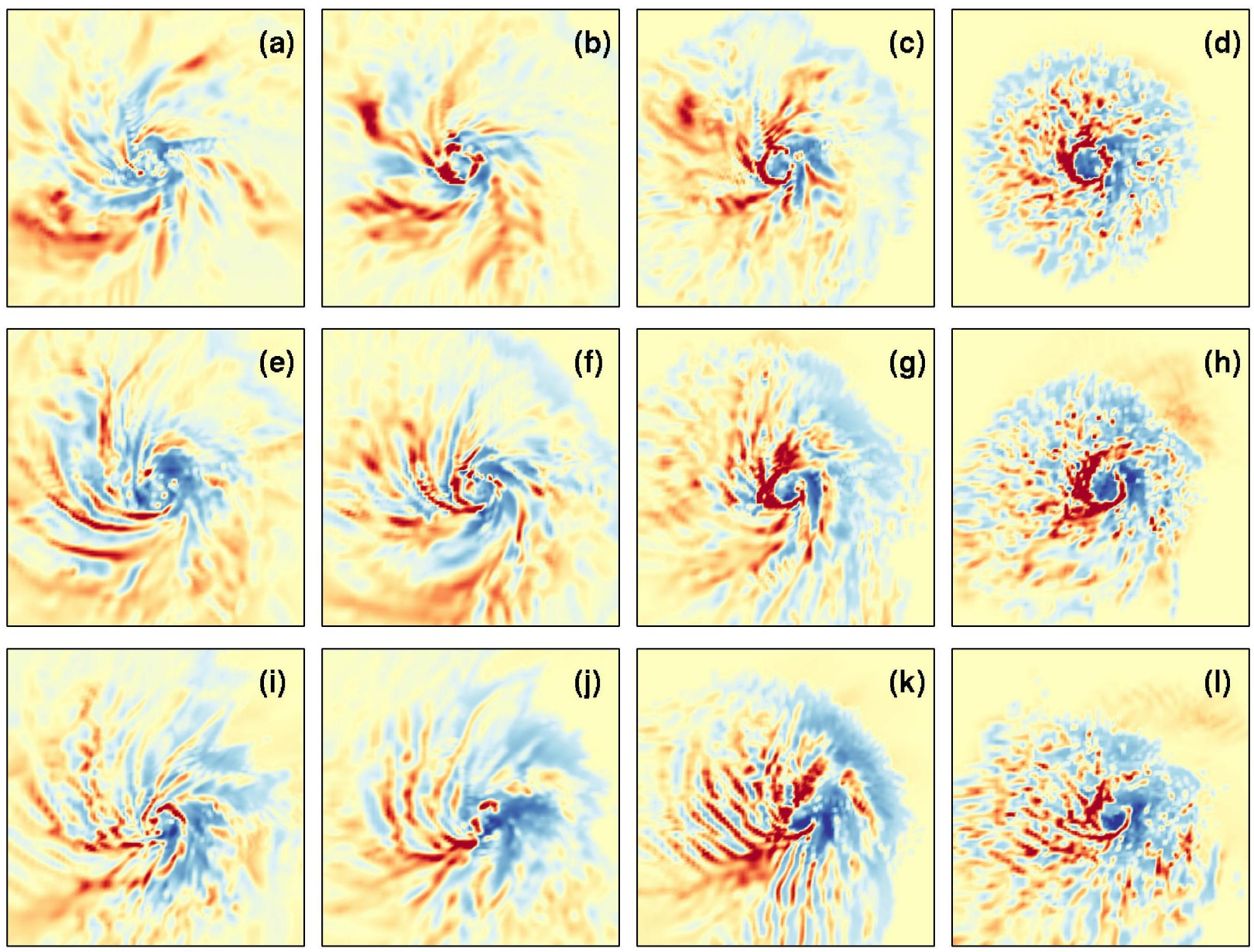

Figure 5.8: As in Figure 5.4 except for the deposition tracks in Figure 5.7. Panels in the middle row are shifted forward by $0.5 R_{c}$ and panels in the bottom row are shifted forward by $R_{c}$. 

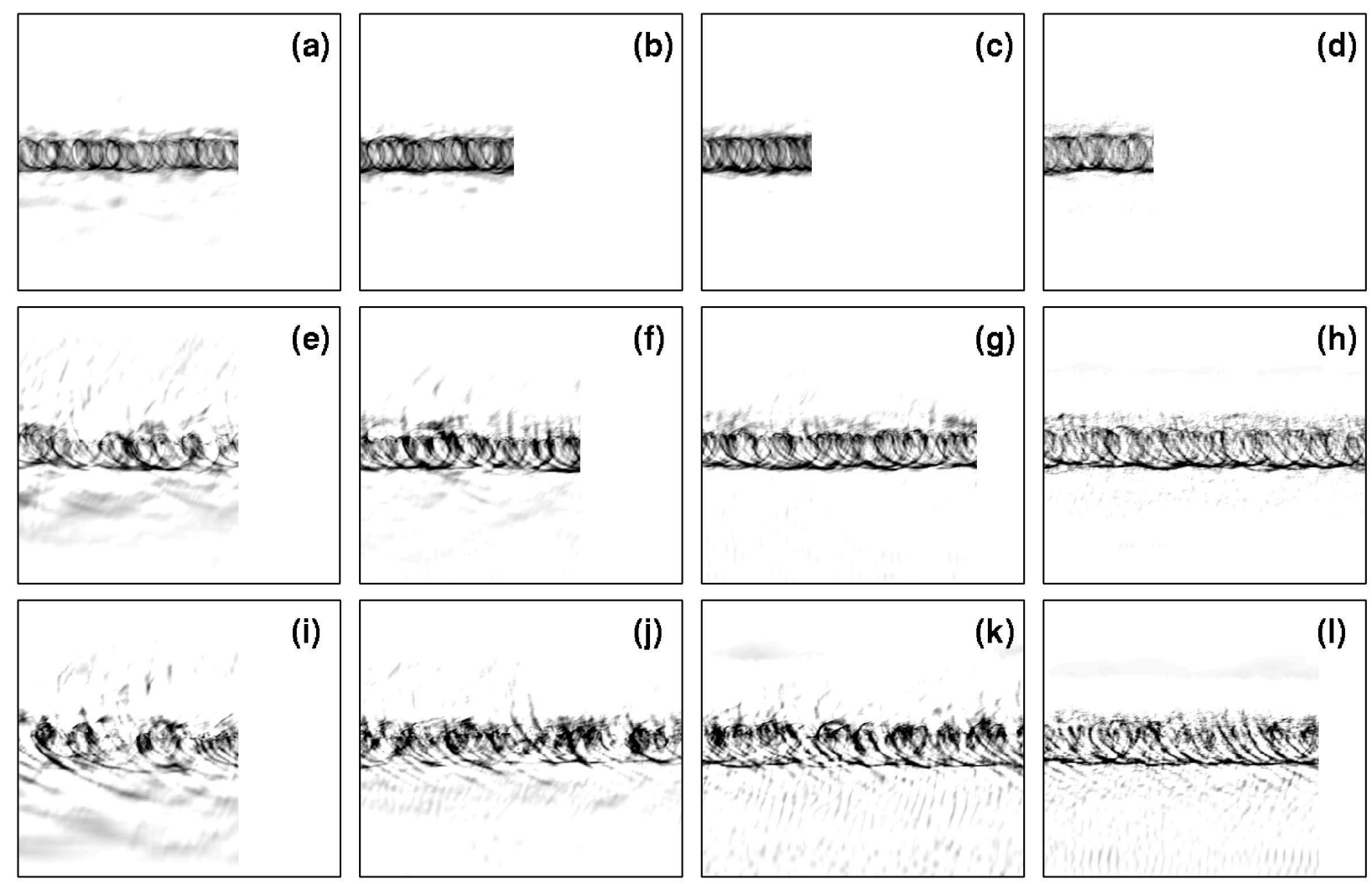

Figure 5.9: Deposition tracks for a collection of tornadoes with $S_{c}=12.4$ and $A_{a}=1.9$, primarily varying $A_{v}$ (left to right) and $A_{t}$ (top to bottom). Left to right: $A_{v}=$ 18.4, 12.2, 8.2, 5.5. Top to bottom: $A_{t}=0.07,0.21,0.36$. Lateral dimensions span $\pm 3 R_{c}$. Grayscale intensities have been scaled as in Figure 5.3; the darkest shades of gray correspond to a dimensionless deposition level of $1.75 \times 10^{3}$. Simulation runs are (a) dmg2177, (b) dmg2059, (c) dmg2080, (d) dmg2090, (e) dmg2178, (f) dmg2014, (g) dmg2081, (h) dmg2091, (i) dmg2179, (j) dmg2082, (k) dmg2092. 

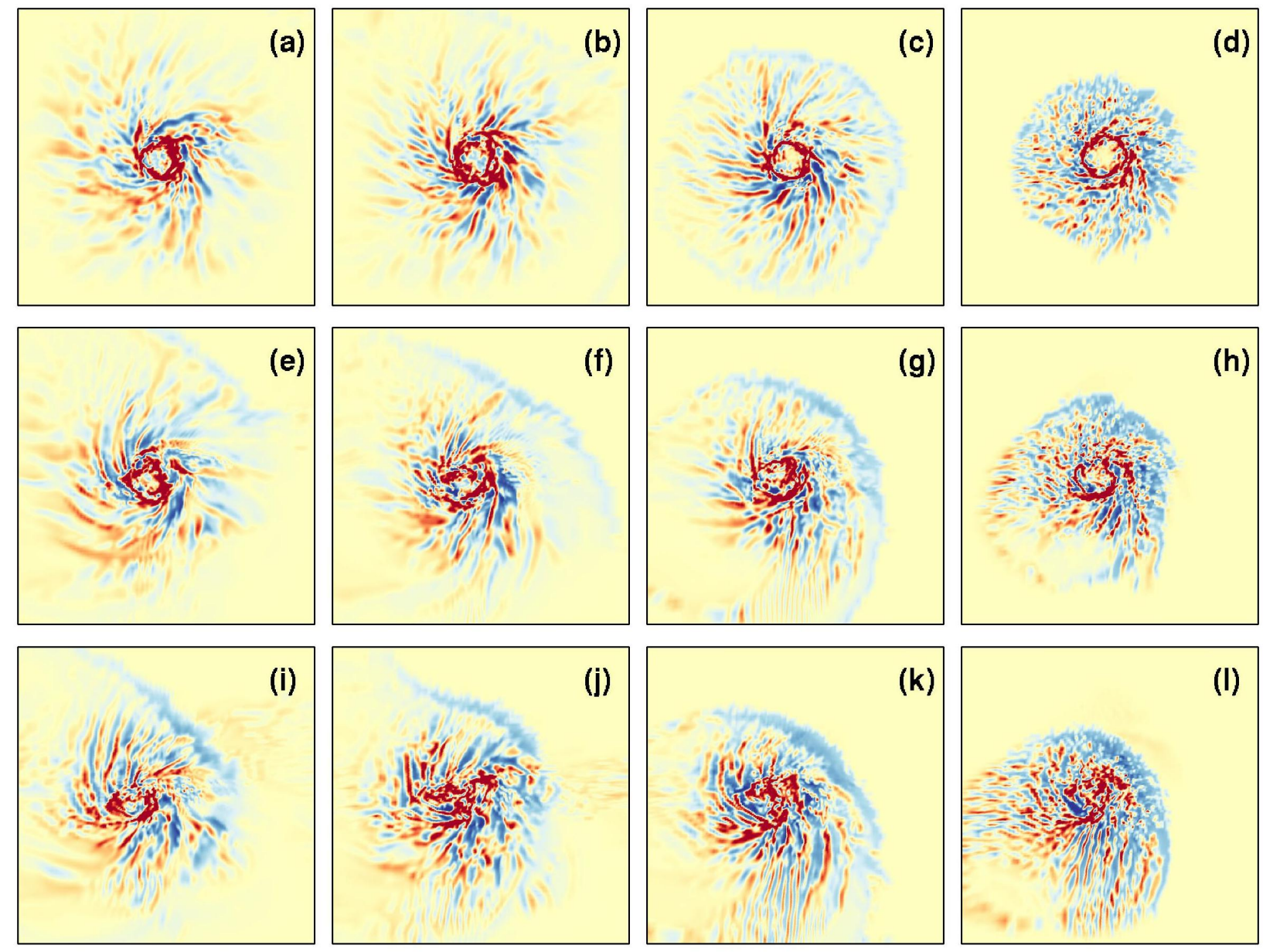

Figure 5.10: As in Figure 5.4 except for the deposition tracks in Figure 5.9. No panels are shifted. 
deposition is not point-like as in Fujita et al.'s model, giving rise to a single line of deposition, but rather loosely annular in structure, giving rise to "loops" or "cycloids" (e.g. flow structure characteristic of Figure 5.11 leads to instantaneous debris flux patterns like those in Figure 5.12b). Critical low-swirl end-wall jet vortices $\left(S_{c}<1.4\right.$, Lewellen et al. (2000)) were found to be generally unable to collect any debris due to their lack of near-surface swirl intensification. Sample cycloids from the literature are shown in Figure 5.13.

As the tornado sweeps across the surface, the resulting deposition patterns are cycloidal, inheriting their overall structure from "translation plus rotation" in the near-surface flow (e.g. the progression in Figure 5.12a,c,e,g). The patterns are broken into distinct swaths due to turbulent fluctuations in debris amount and local flow conditions (provided in part by inflow rolls). If not for these factors, the tornado would simply leave a smeared-out distribution of debris. The strongest marks tend to be associated with regions of convergence along the local streamlines in the surface frame, allowing debris to gather in a line-like structure (e.g. Figure 5.15). Some marks form all at once and others are created as localized packets of debris travel across the surface, depositing their load as they move along.

As translation becomes more significant (i.e. $A_{t}$ is increased), the central annulus is shifted and stretched in non-axisymmetric fashion by friction acting on the near-surface flow (e.g. the progression in Figure 5.12b,d,f,h). This causes the apparent spacing between marks to increase and the left/right asymmetry to become more pronounced (e.g. the progression of Figure 5.12a,c,e,g). The convolution of removal and deposition plays a role in the leftright asymmetry. For example, at higher translation speeds in some cases the near-surface horizontal convergence in the rear part of the annulus is reduced enough to facilitate localized removal, which competes with deposition in the front and right part of the annulus (e.g. the progressions across the bottom row of Figure 5.16 and along the rightmost three columns of Figures 5.35 .4 ). The mean deposition per unit length goes as $S_{c} A_{t}^{-1}$ (e.g. Figure 5.14).

When cycloidal marks are present, the radius of cycloidal deposition $R_{s}$ correlates with the radius of the updraft annulus (and also the radius $R_{l}$ of the maximum near-surface swirl 
velocity). $R_{l} / R_{c}$ increases with $S_{c}$ above the critical low-swirl limit (Lewellen and Lewellen, 2007a; Lewellen et al., 2000), so it is no surprise that $R_{s} / R_{c}$ increases with $S_{c}$ as well (e.g. the progressions across the top rows of Figures 5.3 and 5.5). Also, the lateral shift of cycloidal marks $\delta_{s} / R_{c}$ (e.g. the shift of the vortex in Figure 5.4i) is affected by translation-induced asymmetries and swirl ratio, increasing with $A_{t} / S_{c}$. It must be noted that in many cases the radial extent of cycloidal deposition is readily apparent (e.g. the tracks of Figure 5.9);

however, $R_{s}$ is undefined in many tracks do not exhibit strictly cycloidal deposition (e.g. $5.7 \mathrm{k})$.

Raising $A_{a}$ increases the importance of radial acceleration with respect to gravity, thereby strengthening the radial component of debris slip in the near-surface inflow. This makes it more difficult for debris to reach the central corner flow before being redeposited; increasing $A_{a}$ results in larger $R_{s} / R_{c}$ (e.g. the progressions in Figure 5.17). However, $R_{s}$ is undefined in Figure 5.17f, as trains of linear features are the strongest net deposition signatures.

Fujita et al.'s suction vortex actually represents the low- $S_{c}$ limit in parameter space, where debris is carried inward to the center of the corner flow and dropped in a small spot rather than an annulus (e.g. Figure 5.17d). This limit is unlikely to be realized in the field because the critical low-swirl limit is difficult to maintain in the presence of debris and natural fluctuations in near-surface inflow conditions.

\subsection{Deposition by debris-laden surface rolls}

Fujita and others observed sweeping sets of arcs, or "drift marks" in tornado tracks that were attributed to reorganization of previously-removed debris by trailing inflow winds (Agee et al., 1977; Davies-Jones et al., 1978; Fujita et al., 1976a). A variety of such marks have been observed in the simulations (e.g. Figure 5.19), with surface rolls providing the physical mechanism for creating sharp "trailing arcs". Sample trailing arcs from the literature are shown in Figure 5.18. 


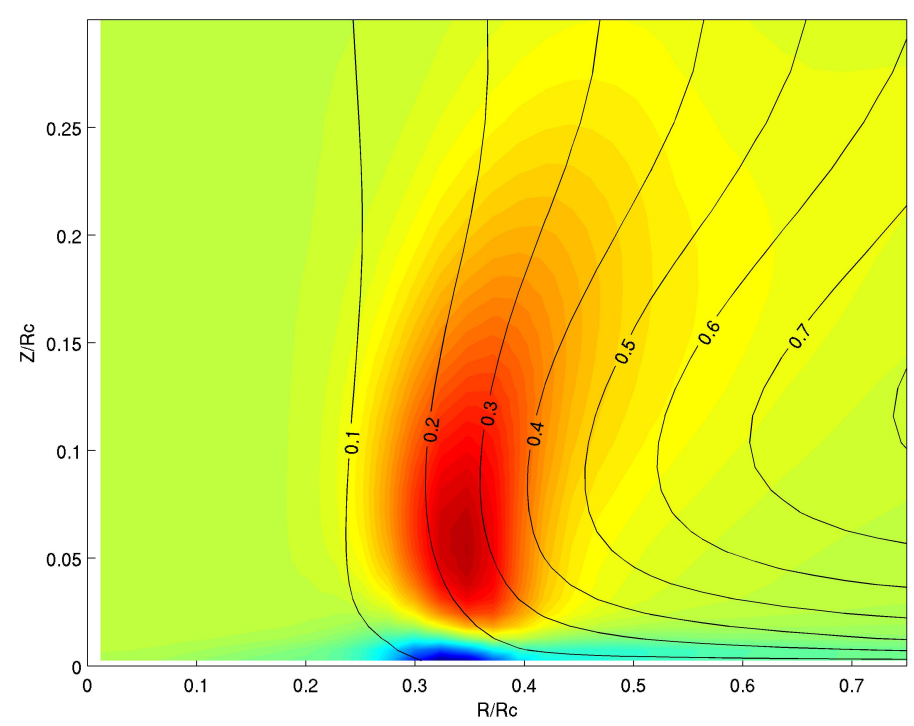

Figure 5.11: Azimuthally averaged vertical debris flux, $W D / V_{c}$ (colors), and angular momentum, $\Gamma / \Gamma_{\infty}$ (contours), from a high-swirl, stationary tornado (run dmg2058). Deep shades of red/blue correpsond to $W D / V_{c}= \pm 0.1$. Note the up/down debris flux couplet in the corner flow; coupled with turbulent variability in debris amount and local flow conditions over time, this general structure is responsible for generating cycloidal marks in translating cases.

With increasing $A_{t}$, the surface layer (with or without debris) gradually transitions from a roughly uniform distribution of turbulent, spiraling inflow rolls into a more-asymmetric distribution (e.g. the progressions along the rows of Figure 5.20). The addition of debris tends to favor rolls along the rear flanks of the tornado (where debris loadings are largest), with the strongest trailing arcs usually originating to the tornado's left (e.g. selected debris fluxes in Figure 5.6). Individual rolls often appear to be stabilized by the presence of debris and sometimes survive long enough to traverse behind and to the right of the central vortex.

Increasing $A_{v}$ typically produces a wider debris-laden inflow, creating longer arcs to the left of the tornado, while lowering $A_{v}$ generates a smaller area of removal that encourages more-central trailing arcs. In many cases, quite linear sets of inflow marks were also observed in the tornado's right flank (e.g. Figures 5.4l, 5.6f,g,h, among others) although their physicality is called into question due to grid-resolution concerns (e.g. Section 3.4.2). Trailing arcs become more pronounced with increasing $A_{a}$ (c.f. Figures 5.3 and 5.5. 

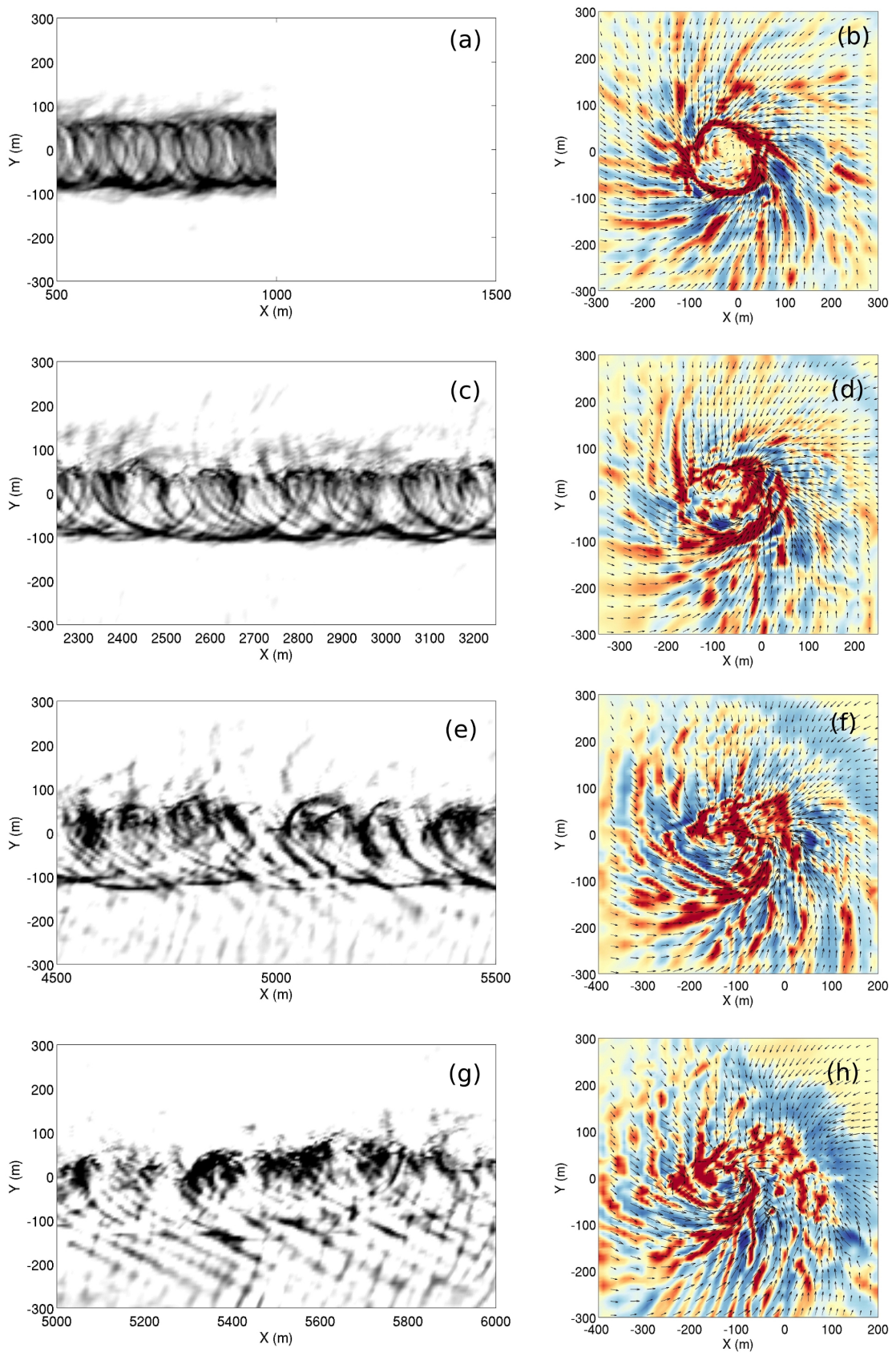

Figure 5.12: Sample cycloidal marks (left column) and sample instantaneous debris flux at the surface (right column) for a collection of high-swirl tornadoes moving at different speeds. Top to bottom: $5 \mathrm{~m} \mathrm{~s}^{-1}$ (dmg2080), $15 \mathrm{~m} \mathrm{~s}^{-1}$ (dmg2081), $25 \mathrm{~m} \mathrm{~s}^{-1}$ (dmg2082), $35 \mathrm{~m} \mathrm{~s}^{-1}$ (dmg2084). Grayscale levels in the left column have been normalized to $U_{\text {trans }}$; the darkest shades of gray in panel (a) correspond to a deposition level of $120 \mathrm{~kg} \mathrm{~m}^{-2}$. Vector fields in the right column represent the horizontal air flow taken in the surface frame at $z=0.5 \mathrm{~m}$, and deep red/blue correspond to deposition/removal of magnitude $2 \mathrm{~kg} \mathrm{~m}^{-2} \mathrm{~s}^{-1}$. 

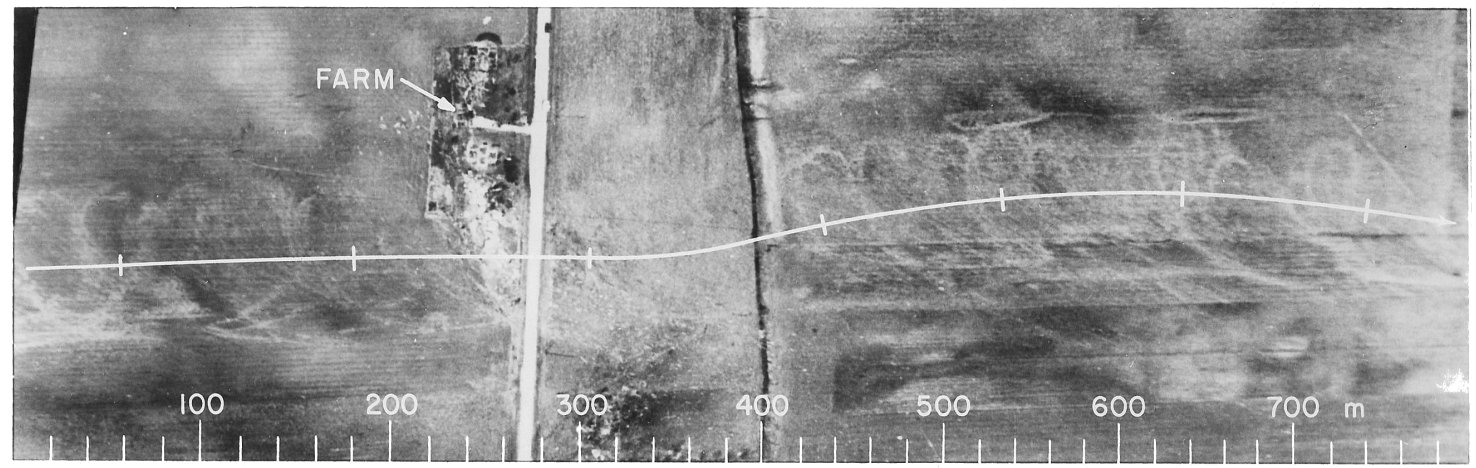

Figure 5.13: Aerial imagery of a tornado damage track, showing a qualitative change in cycloidal marks from left to right across the image. From Fujita (1967a)

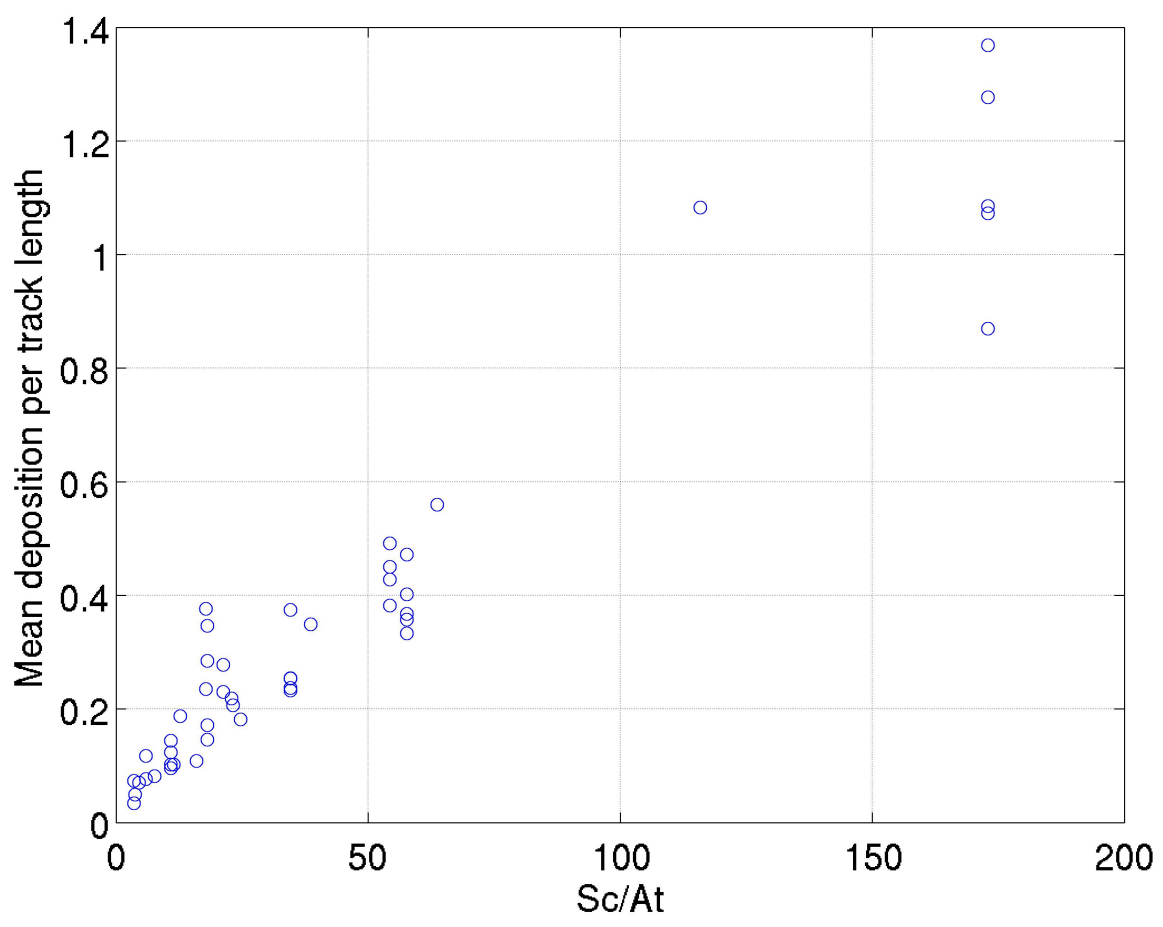

Figure 5.14: Mean deposition level (nondimensionalized by air density $\rho$ ) versus $S_{c} A_{t}^{-1}$. 


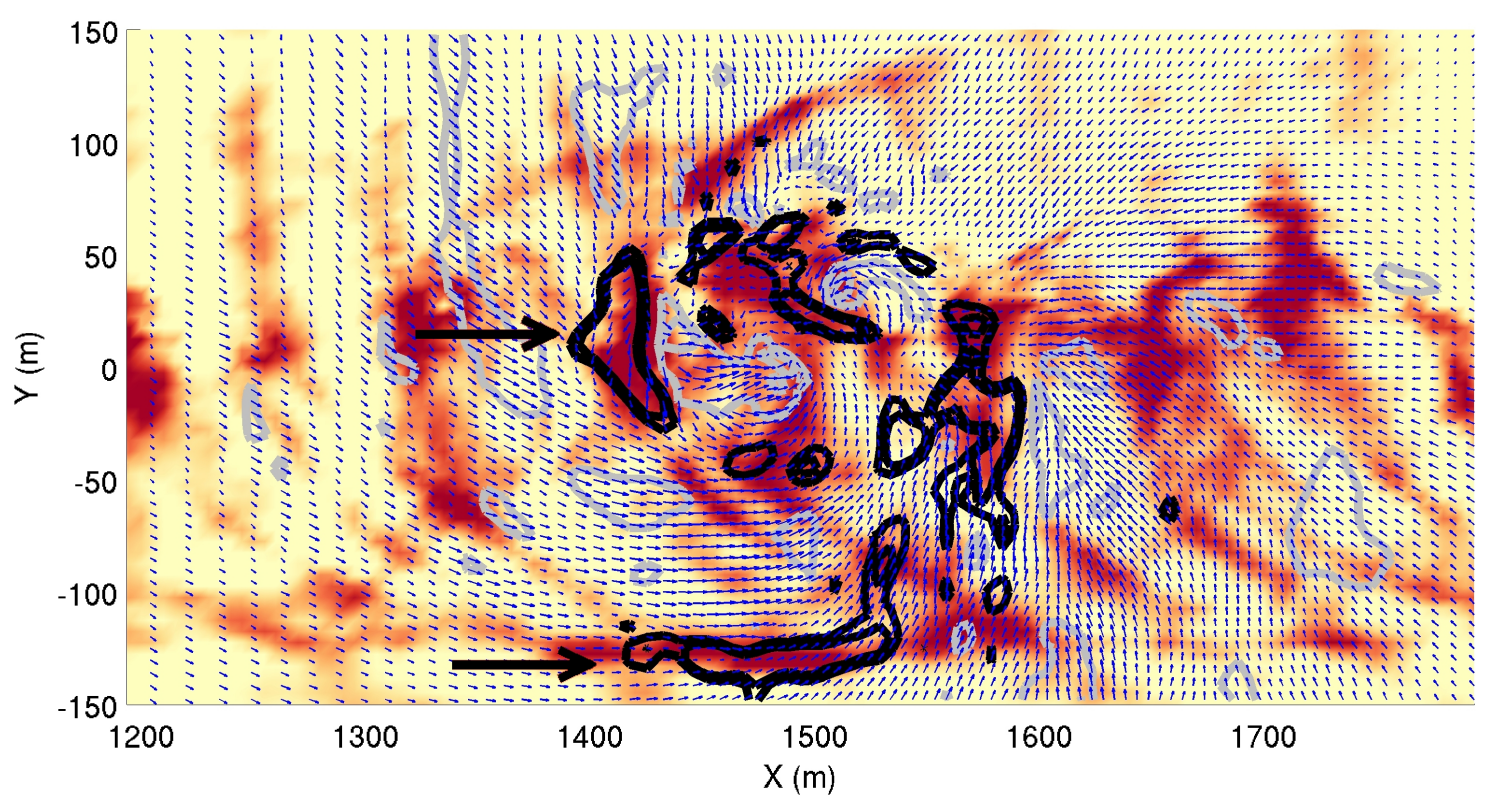

Figure 5.15: Illustration of strong deposition marks being laid down in regions of convergence along the local streamlines from simulation dmg2060 (the black arrows indicate the features of interest). Colors: net deposition after the tornado has passed (deep red corresponds to a deposition level of $30 \mathrm{~kg} \mathrm{~m}^{-2}$ ). Vectors: instantaneous horizontal air flow at $z=0.5 \mathrm{~m}$. Contours: instantaneous downward debris flux (black/gray correspond to downward/upward debris fluxes of respective peak values $-6 \mathrm{~kg} \mathrm{~m}^{-2} \mathrm{~s}^{-1}$ and $+4 \mathrm{~kg} \mathrm{~m}^{-2} \mathrm{~s}^{-1}$ ).

Some evidence is provided in the literature for the role played by inflow rolls in modulating debris fluxes throughout the surface layer. Davies-Jones et al. (1978) observed coherent $\sim 1$ m-scale rows of alternating convergence and divergence in the orientations of wheat after a tornado had passed (e.g. Figure 5.21), and postulated that these patterns were generated by shallow horizontal roll vortices; these patterns closely resemble wind patterns observed in the vicinity of simulated inflow rolls on spatial scales an order of magnitude larger. They also noted removal of wheat in regions of cross-stream divergence which is generally consistent with debris removal by inflow rolls in the simulations (e.g. Figure 5.22). If the alternating regions of convergence and divergence had been correlated with the rear part of the tornado then it would be likely that these features had been generated by the same class of inflow rolls that give rise to trailing arcs (although the current simulation set does not explicitly resolve $1 \mathrm{~m}$-scale flow features). However, the lack of any particular vortexrelative orientation suggests that these marks may have been created by transient zones 

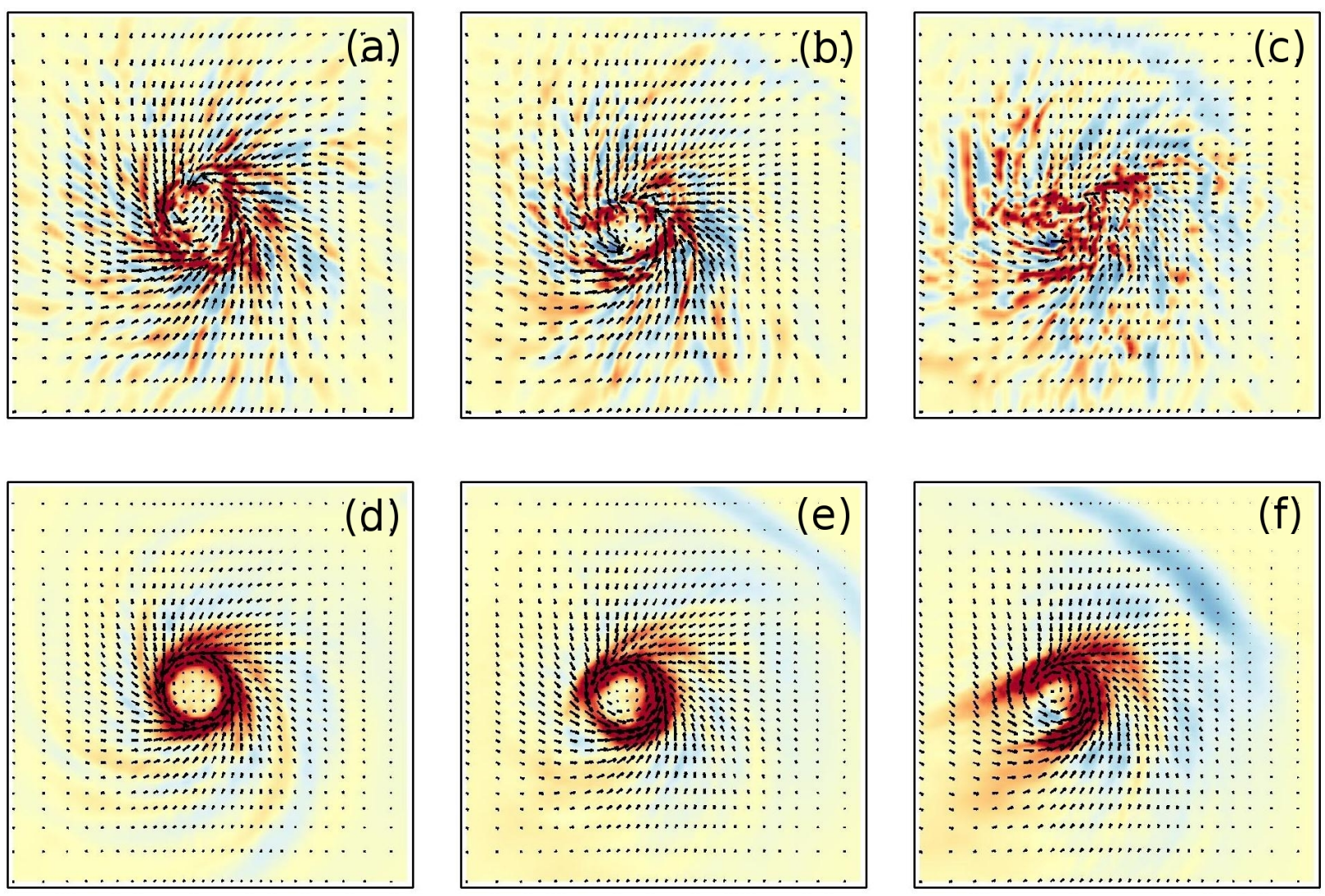

Figure 5.16: Instantaneous and time-averaged surface debris fluxes (top and bottom rows, respectively) for a collection of high-swirl tornadoes. Left to right: $5 \mathrm{~m} \mathrm{~s}^{-1}$ (dmg2059), 15 $\mathrm{m} \mathrm{s}^{-1}$ (dmg2014), and $25 \mathrm{~m} \mathrm{~s}^{-1}$ (dmg2060) vortex translation. Deep red/blue correspond to deposition/removal levels of magnitude $3 \mathrm{~kg} \mathrm{~m}^{-2} \mathrm{~s}^{-1}$ (top row) and $1.5 \mathrm{~kg} \mathrm{~m}^{-2} \mathrm{~s}^{-1}$ (bottom row). 

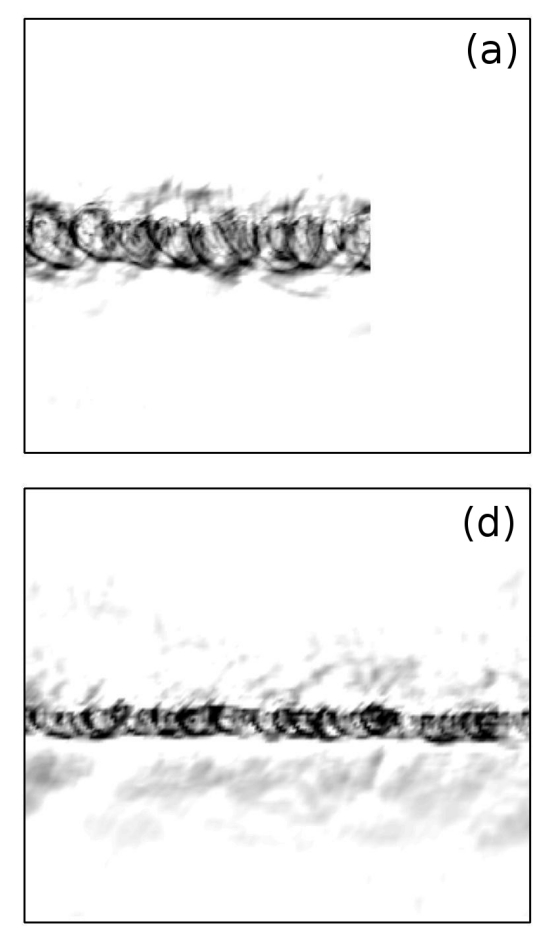
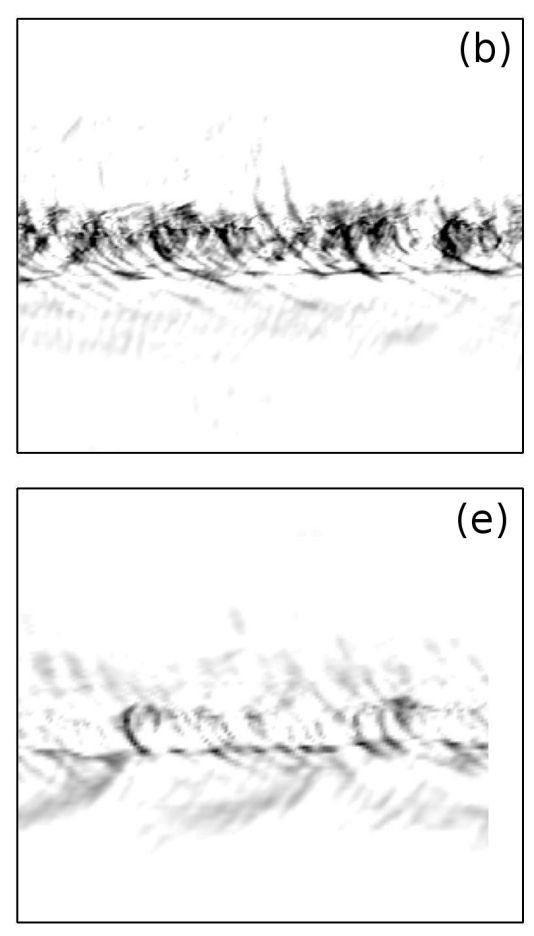
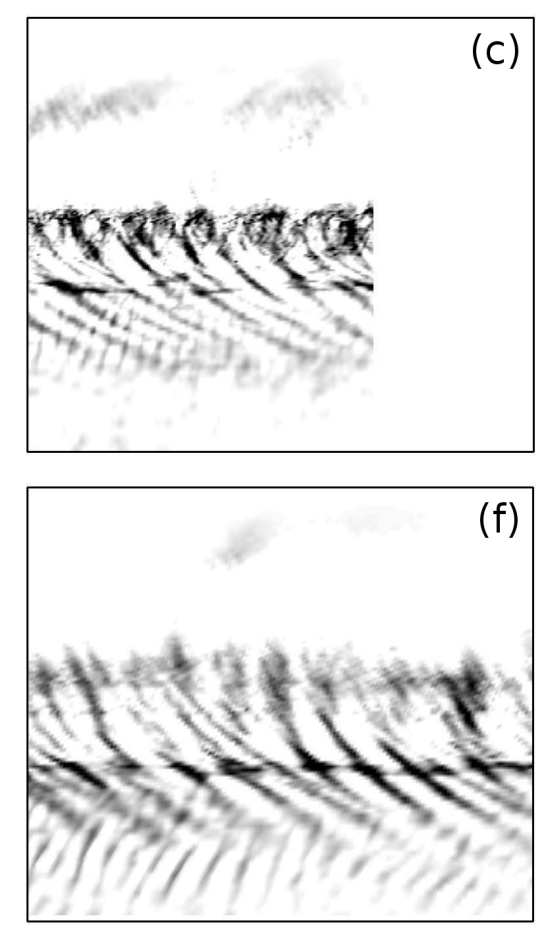

Figure 5.17: Deposition tracks showing variation with $A_{a}$ within two collections of tornadoes. Top row: high swirl $\left(S_{c}=12.4, A_{t}=0.36, A_{v}=10.2,12.2,12.3, A_{a}=0.5,1.9,7.9\right)$; bottom row: medium-swirl $\left(S_{c}=3.0, A_{t}=0.278,0.278,0.333, A_{v}=10.2,10.3,10.5\right.$, $A_{a}=0.5,1.9,7.8$ ). Simulation runs are (a) dmg2280, (b) dmg2060, (c) dmg2195, (d) dmg2279, (e) dmg2203, (f) dmg3026. 


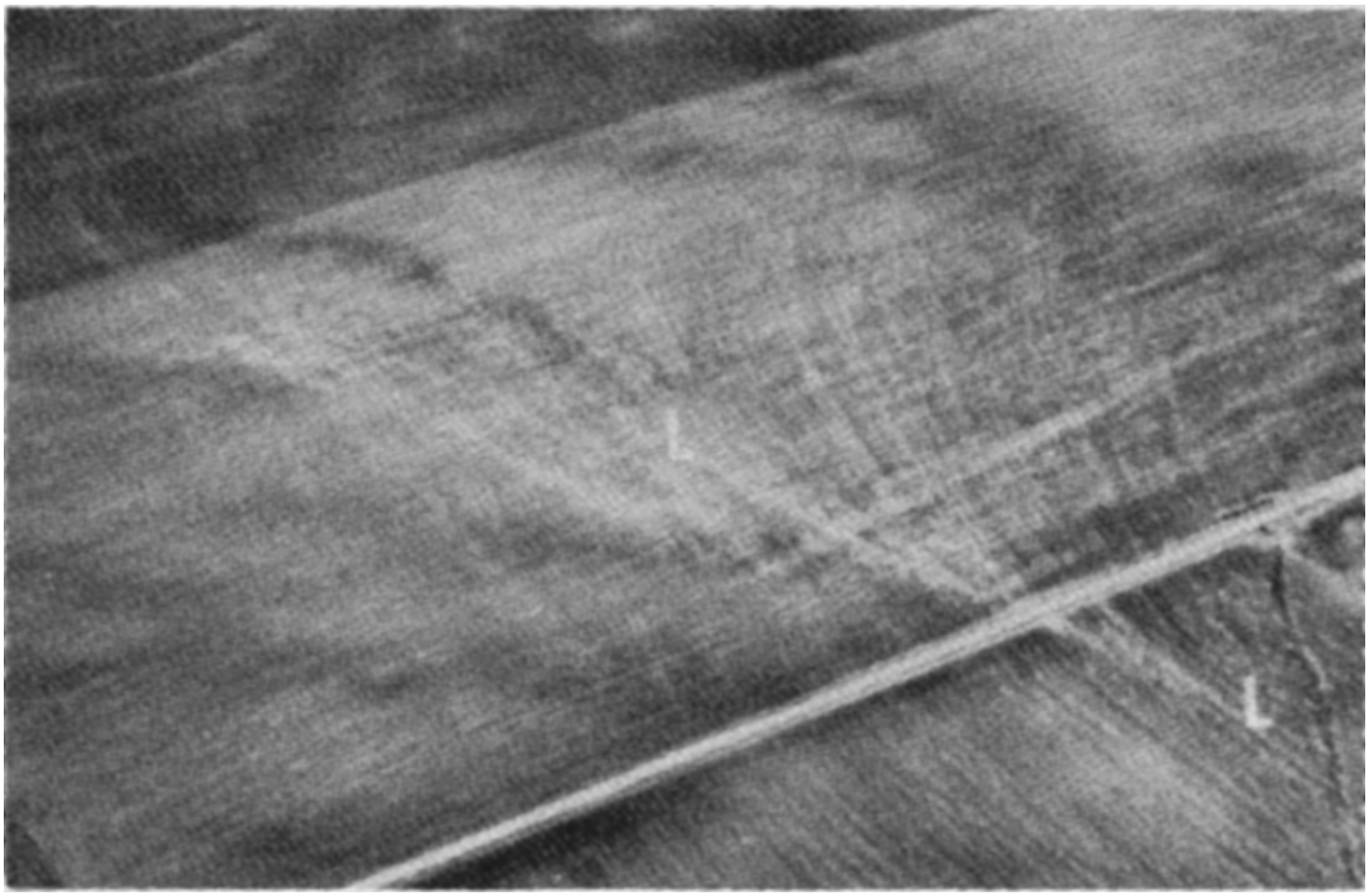

Figure 5.18: Aerial imagery of a tornado damage track from Fujita et al. (1976b), showing trailing arcs.

of alternating convergence and divergence (correlated with updraft/downdraft air velocity couplets) elsewhere in the inflow layer.

\subsection{Deposition of fully lofted debris}

Large deposition swaths far from the central vortex, or "diffuse far-field swaths" are here attributed to deposition of debris that has been fully lofted and carried outward (e.g. Figure 5.23). It is unclear whether far-field swaths have been observed in the field. The signatures characterized as "drift marks" in Figure 5.24 may actually be far-field deposits.

Dowell et al. (2005) observed "debris curls" in photographs of a real tornado where debris has exited the central updraft and begun its descent back to the ground (Dowell et al., 2005); this type of flow feature could lead to far-field marks as long as the debris packet is not reinjected into the surface inflow or deposited and re-removed. Debris curls 

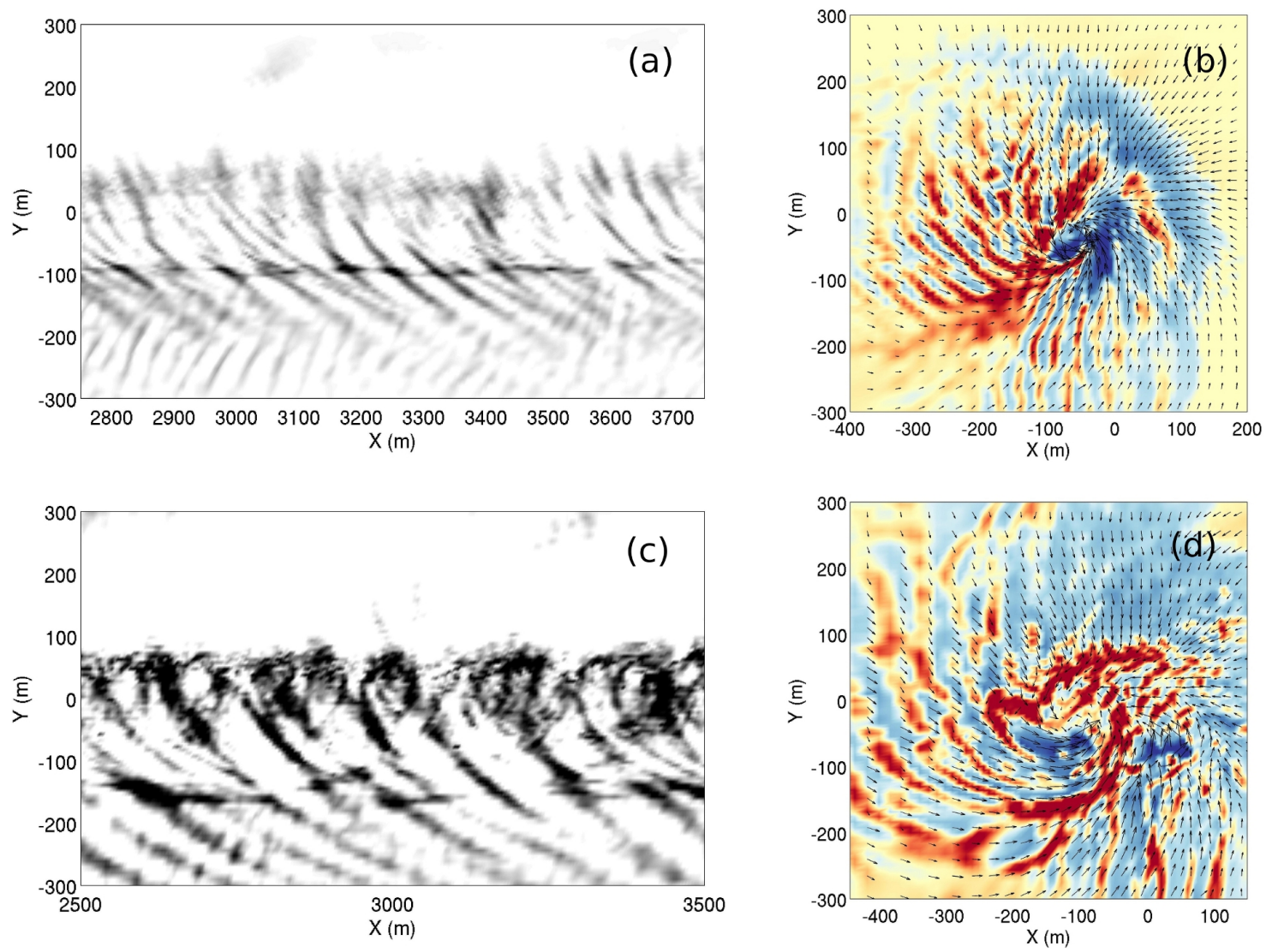

Figure 5.19: Sample trailing arcs (left column) and sample instantaneous debris flux at the surface (right column) for a collection of tornadoes moving at $25 \mathrm{~m} \mathrm{~s}^{-1}$. Top row: mediumswirl (run dmg3026); bottom row: high-swirl (run dmg2195). The darkest shades of gray correspond to a deposition level of $30 \mathrm{~kg} \mathrm{~m}^{-2}$. Vector fields in the right column represent the horizontal air flow taken in the surface frame at $z=0.5 \mathrm{~m}$, and deep red/blue correspond to deposition/removal of magnitude $2 \mathrm{~kg} \mathrm{~m}^{-2} \mathrm{~s}^{-1}$. 

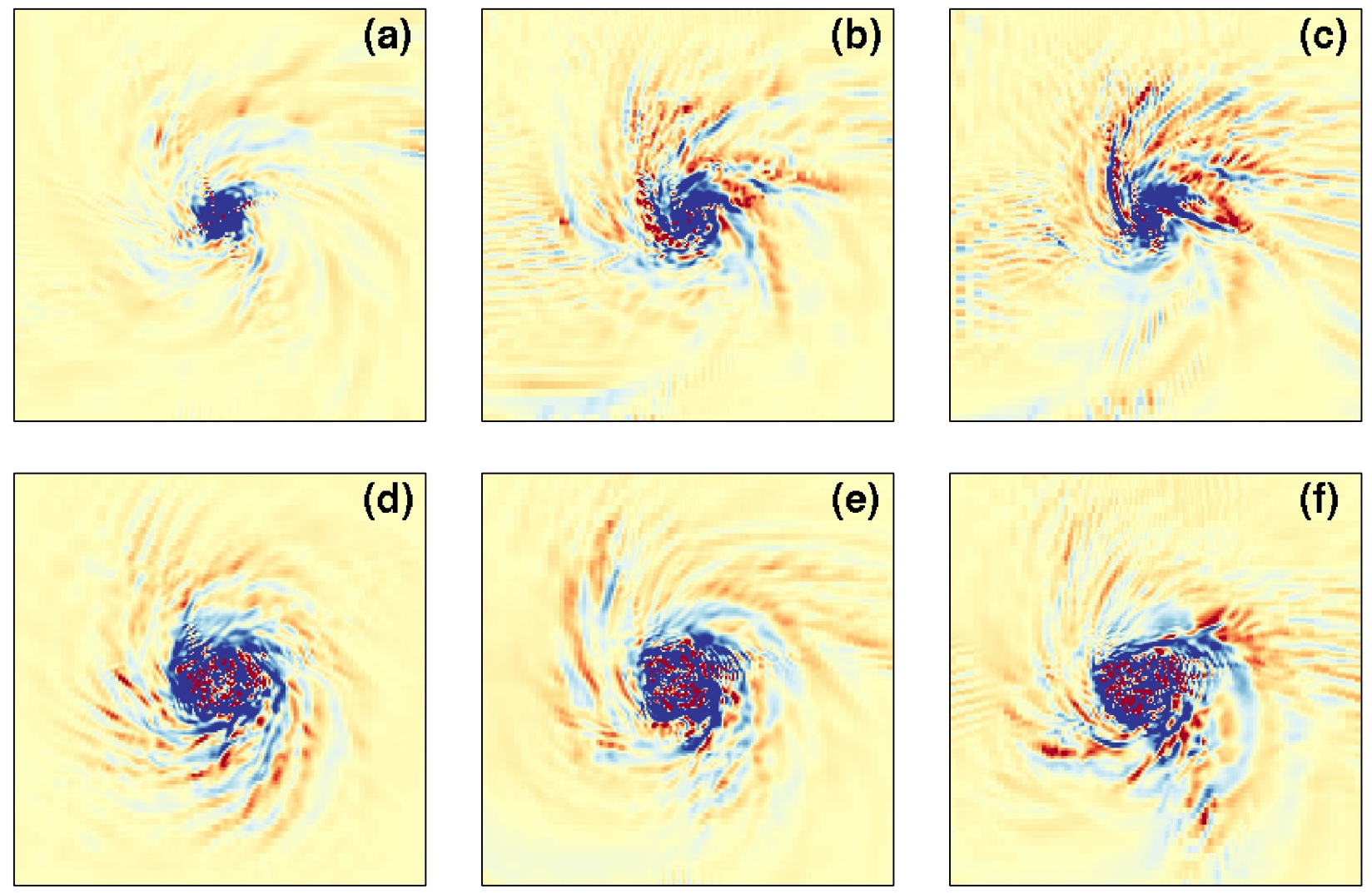

Figure 5.20: Horizontal slices of instantaneous vertical air velocity at $\mathrm{z}=1.0 \mathrm{~m}$, showing existence of near-surface inflow rolls (up/down velocity couplets) in the absence of debris. Red/blue represent downward/upward velocities of magnitude $0.5 \mathrm{~m} \mathrm{~s}^{-1}$. Top row: mediumswirl tornadoes moving to the right at (a) $5 \mathrm{~m} \mathrm{~s}^{-1}$ (run2049), (b) $15 \mathrm{~m} \mathrm{~s}^{-1}$ (run2011), and (c) $25 \mathrm{~m} \mathrm{~s}^{-1}$ (run2052). Bottom row: high-swirl tornadoes moving to the right at (d) $5 \mathrm{~m}$ $\mathrm{s}^{-1}$ (run2059), (e) $15 \mathrm{~m} \mathrm{~s}^{-1}$ (run2014), and (f) $25 \mathrm{~m} \mathrm{~s}^{-1}$ (run2060). Spatial dimensions span $\pm 600 \mathrm{~m}$. 


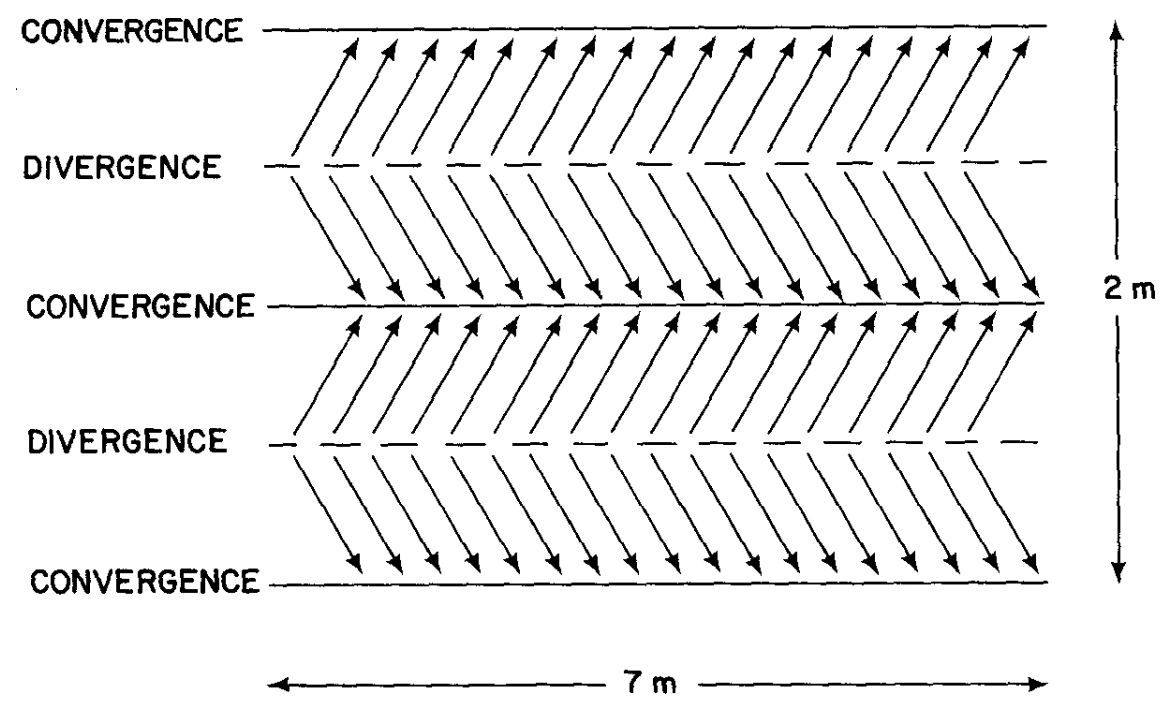

Figure 5.21: Convergence/divergence pattern observed in tall wheat stubble by DaviesJones et al (Davies-Jones et al., 1978). Comparable with rows of alternating convergence/divergence in Figure 5.22; however the wheat stubble patterns showed no directional preference with respect to the central vortex and were of smaller scale.

have been observed in the simulations.

Raising the parameter $A_{v}$ increases the intensity of diffuse far-field swaths with respect to cycloids and trailing arcs (e.g. the progression across the top row of Figure 5.7). More specifically, increasing $A_{v}$ decreases the maximum possible amount of debris slip, which enables a larger fraction of debris to follow the corner flow streamlines. A higher amount of lofted debris produces a more-massive debris cloud which leads to stronger deposition in the far-field (Lewellen et al., 2008). Also, fully lofted debris acquires some vertical slip velocity when approaching the ground; vertical air velocities go to zero at the surface, and each debris packet must continue to the surface with finite velocity in order to be redeposited.

Asymmetric deposits of debris (typically right and rearward) result from tilting of the main vortex right and rearward with respect to the vortex center aloft. That is, debris vertical velocities typically point downward where the debris cloud meets the surface behind and to the right of the main vortex (e.g. Figures 5.4a, 5.6a, 5.8a, among others); this process has been observed in animations of simulation data regarding debris cloud formation. Leftward piles of debris occur in regimes where the surface flux of lofted debris outweighs removal by 


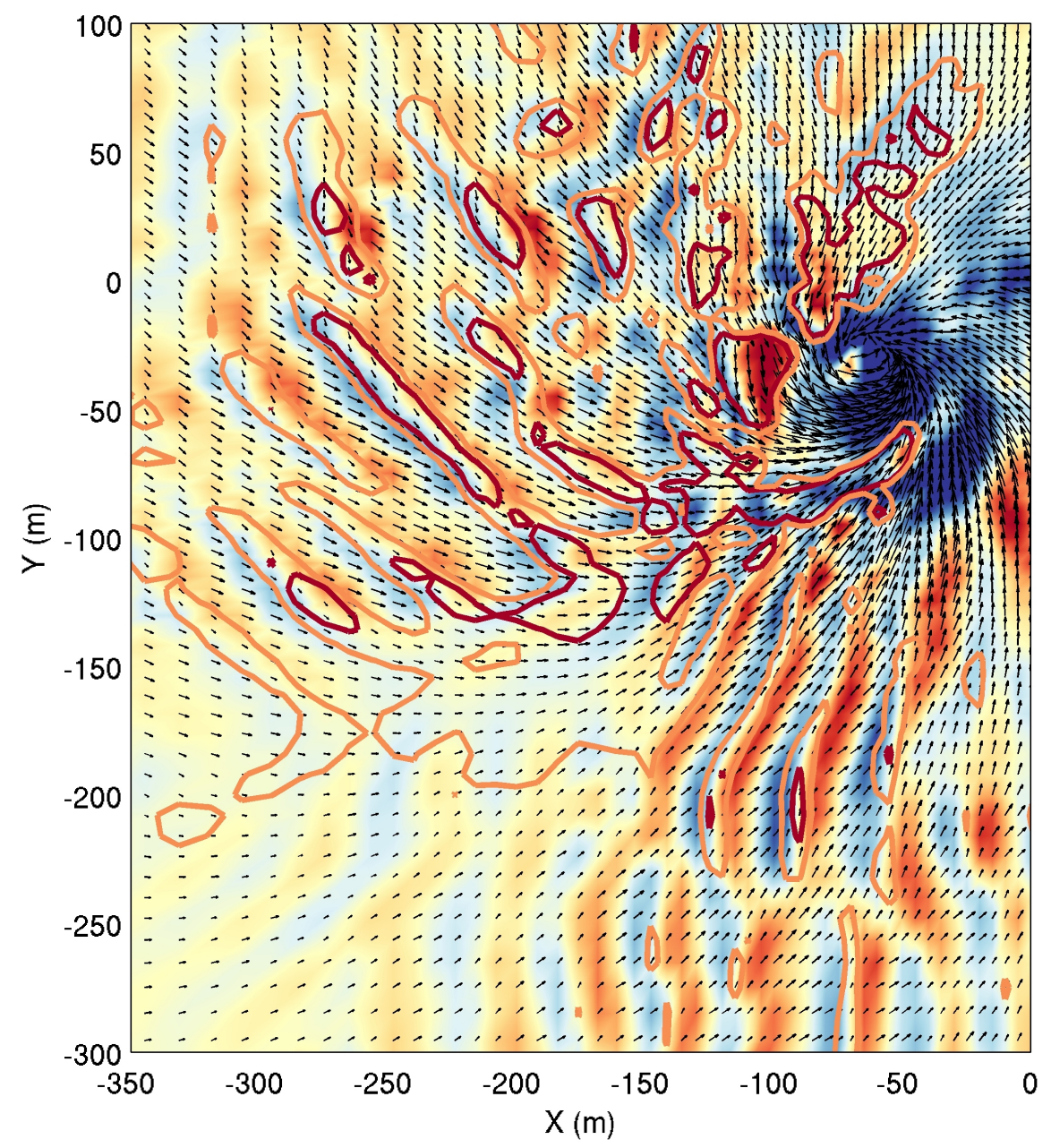

Figure 5.22: Instantaneous snapshot of surface-relative horizontal flow from medium-swirl run dmg3026 at $z=0.5 \mathrm{~m}$ (vectors) and vertical velocity at $z=1.0 \mathrm{~m}$ (colors; red and blue represent, respectively, convergence and divergence). Contour lines represent instantaneous debris flux, with deep red representing a downward flux of magnitude $2 \mathrm{~kg} \mathrm{~m}^{-2} \mathrm{~s}^{-1}$. Debris deposition occurs near to regions of horizontal convergence, athough deposition is typically offset from the locations of peak convergence. 

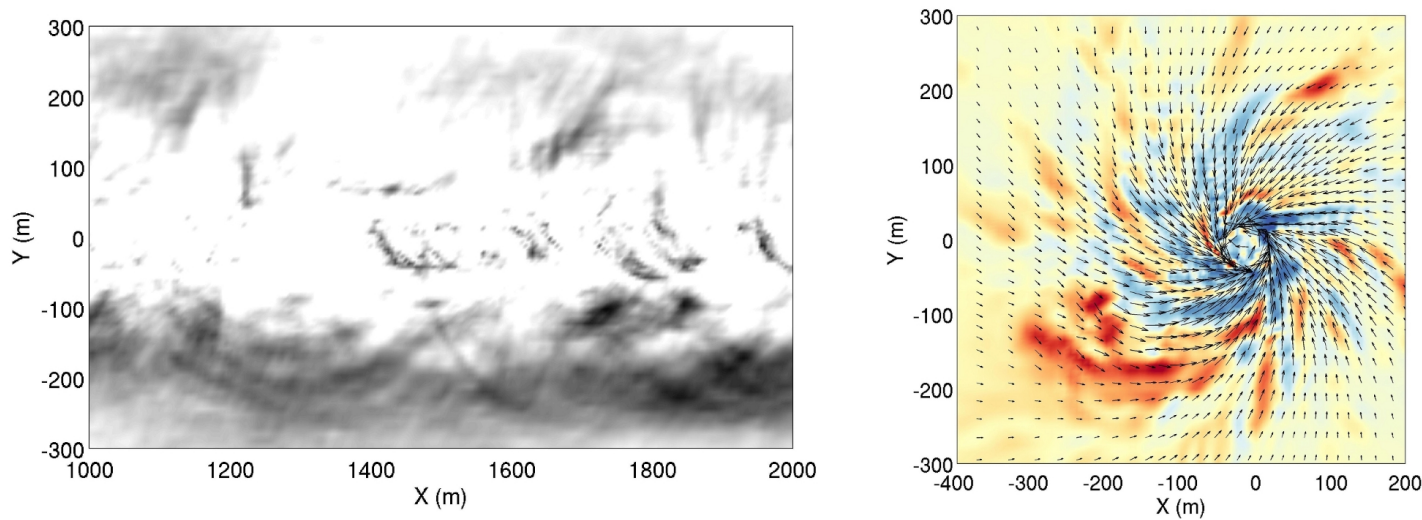

Figure 5.23: Sample diffuse far-field swaths (a) and sample instantaneous debris flux at the surface (b) for a medium-swirl tornado moving at $5 \mathrm{~m} \mathrm{~s}^{-1}$ (run dmg3031). The darkest shades of gray correspond to a deposition level of $45 \mathrm{~kg} \mathrm{~m}^{-2}$. Vector fields in the right column represent the horizontal air flow taken in the surface frame at $z=0.5 m$, and deep red/blue correspond to deposition/removal of magnitude $2 \mathrm{~kg} \mathrm{~m}^{-2} \mathrm{~s}^{-1}$.

the inflow layer (e.g. Figure 5.5b,d).

\subsection{Effects of debris availability}

Debris availability plays a strong role in asymmetry of surface tracks. At very low availability (as opposed to unlimited availability in the majority of simulated cases) only a subset of cycloidal marks or far-field swaths to the righthand side of the tornado are present (e.g. compare Figures 5.25a,b). Deposition to the right side of the vortex is most robust to changes in availability because it is often a region of relatively sustained convergence along the local surface-frame streamlines. Fujita instead attributed this "scalloping" subset of marks to secondary vortices rotating slowly around the central vortex.

Decreasing $A_{v}$ also imposes a constraint on the effective surface debris availability (increasing $w_{t}$ increases the threshold for removal and decreasing $V_{c}$ lowers the ability of the near-surface flow to remove debris). The scalloping limit is approached as $A_{v}$ is decreased in the presence of significant translation, eventually giving way to linear or dash-like deposition marks. For example, in the high-swirl sequence of runs across the bottom row of Figure 


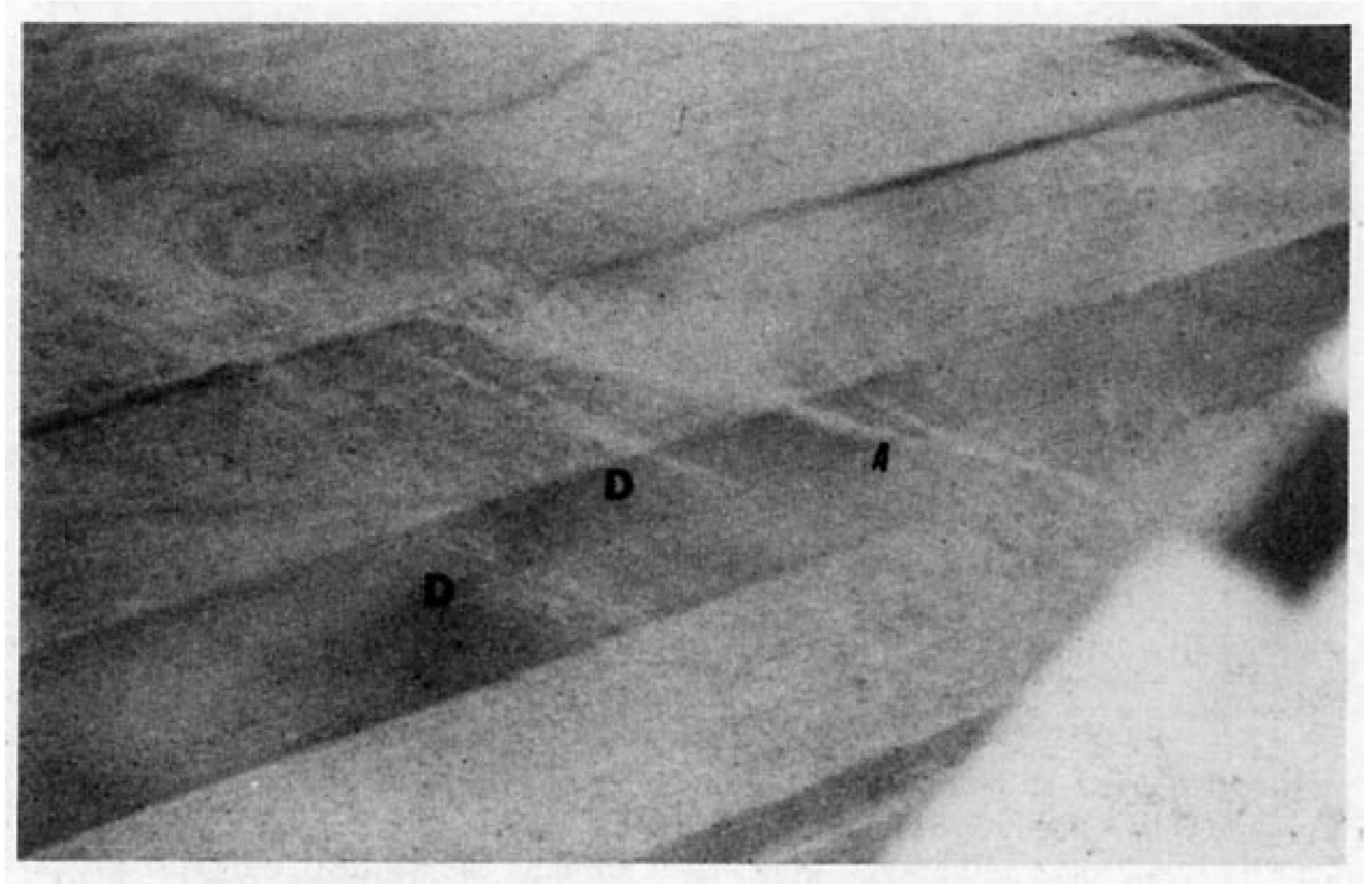

Figure 5.24: Aerial imagery of a tornado damage track from Agee (1977), showing possible diffuse far-field swaths perhaps mischaracterized as drift marks (D).

5.9, deposition under the righthand side of the vortex becomes stronger as $A_{v}$ is decreased (e.g. also illustrated by the progression of the righthand deposition peak in Figure 5.26). Deposition to the lefthand side of the vortex is intermittent at $A_{v}=18.4$ (giving it a weak downstream signature) but becomes more regular at $A_{v}=12.2$ and $A_{v}=8.2$, finally waning in relative intensity (with respect to trailing arcs) at $A_{v}=5.5$ (e.g. also illustrated by the progression of the lefthand peak in Figure 5.26). In the low-swirl regime, the vortex tilt produces strong removal to the front and left of the tornado, often favoring scalloping on the righthand side of the vortex (e.g. Figure 5.3a).

Fujita interpreted linear marks, or "lineation", as deposition underneath the center of a single translating vortex. Instead, simulation results suggest that in the medium- and highswirl regimes the effect that Fujita called "lineation" is essentially identical to scalloping.

In the high-swirl, extremely low- $A_{v}$ limit, the only features capable of removing debris from the surface are secondary vortices (e.g. Figure 5.27a). This regime is perhaps most 

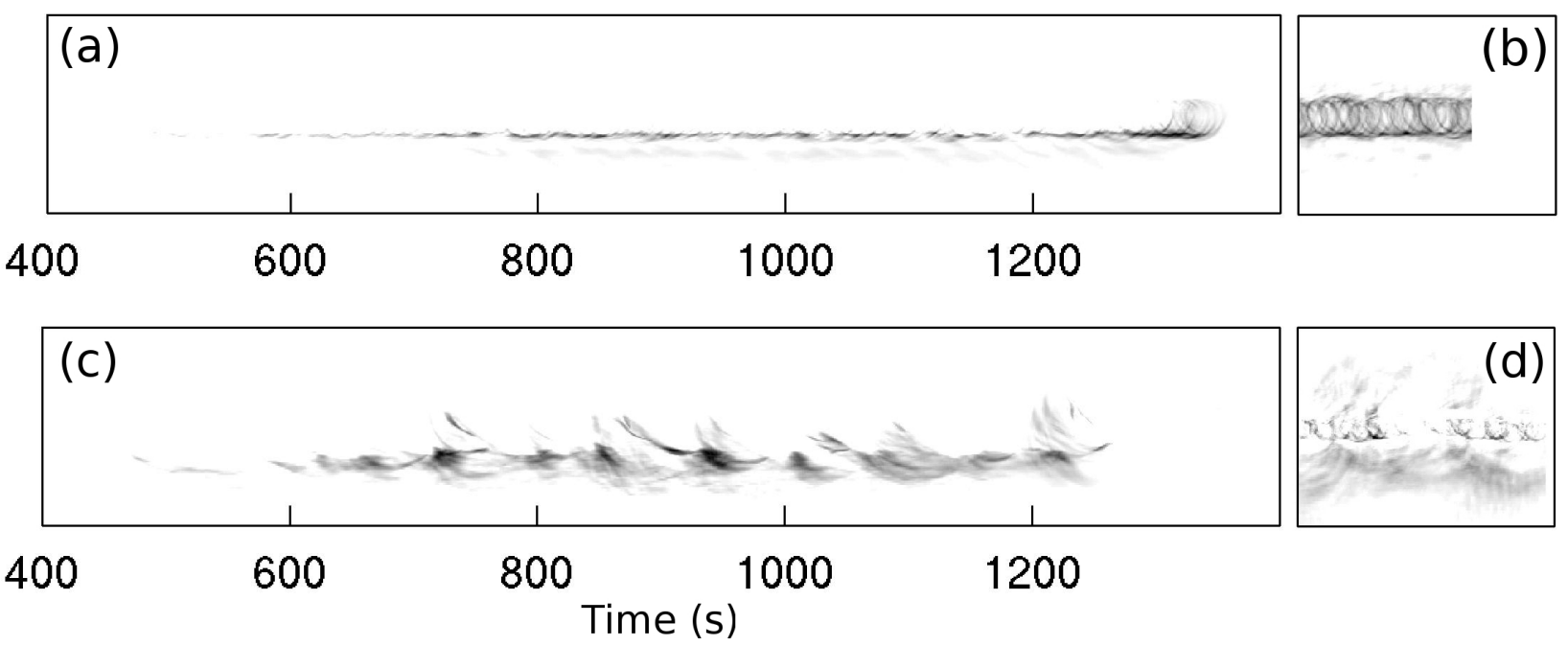

Figure 5.25: Deposition tracks for simulated tornadoes moving at $5 \mathrm{~m} / \mathrm{s}$ over a limited (1 $\mathrm{kg} \mathrm{m}^{-2}$ ) surface debris source (left column) and an unlimited surface debris source (right column). (a,b) High-swirl cases $\left(S_{c}=12.4, A_{t}=0.072, A_{v}=8.2, A_{a}=2.4\right.$ ). (c,d) mediumswirl cases $\left(S_{c}=3.0, A_{t}=0.055, A_{v}=15.9, A_{a}=7.9\right)$. Simulation runs are (a) dmg2165, (b) dmg2059, (c) dmg2209, (d) dmg2049.

relevant to urban settings, where there is limited, heavy debris, although it would be extremely difficult to infer tornado structure from an urban surface track due to the effects of large man-made obstructions to the flow. However, the extremely low- $A_{v}$ limit may sometimes produce "effective" surface marks for a tornado moving over a crop that is rooted strongly in the ground; the resulting patterns of bent/sparsely removed stalks could provide an indication of the paths of secondaries.

\subsection{Time-varying cases}

Figure 5.28 shows deposition tracks from a collection of four evolving "corner flow collapse" cases (Lewellen and Lewellen, 2007b). The corner flow collapse driving the nearsurface vortex intensification is triggered purely by perturbing the low-level inflow over a single quadrant of the outer domain boundary. The cases differ in the choice of quadrant; the symmetry between the choices is broken by the slow translation velocity of the parent vortex. Dramatic changes in the magnitude, onset and duration of the vortex intensification 


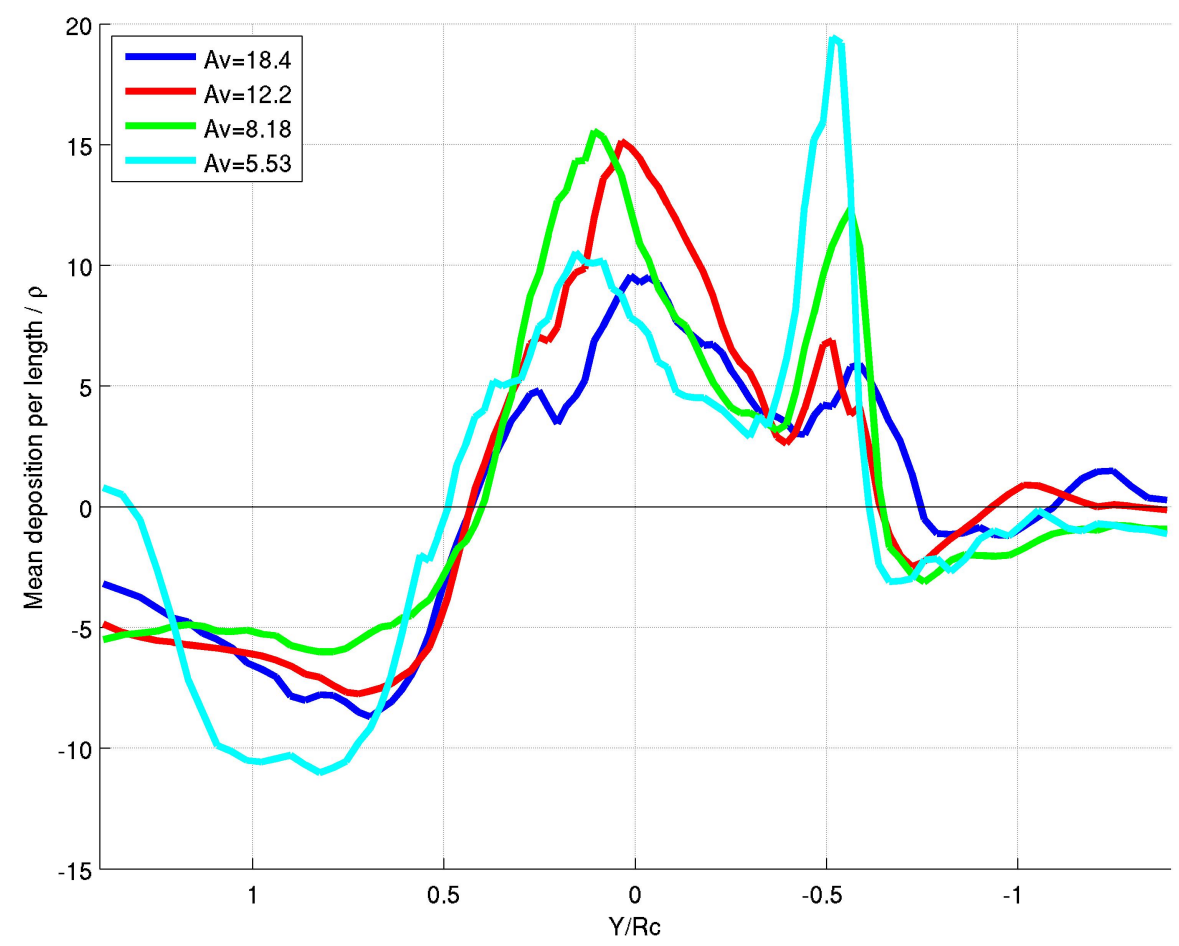

Figure 5.26: "Downstream averages", or the lateral of profile mean deposition per length, from a collection of high-swirl tornadoes with $A_{t}=0.36$ and different $A_{v}$. Note the change in relative deposition level between the double peaks with decreasing $A_{v}$ (the righthand peak dominates at low $A_{v}$, which is the "scalloping" limit). In order of decreasing $A_{v}$, the simulation runs are dmg2179, dmg2060, dmg2082, dmg2092. 

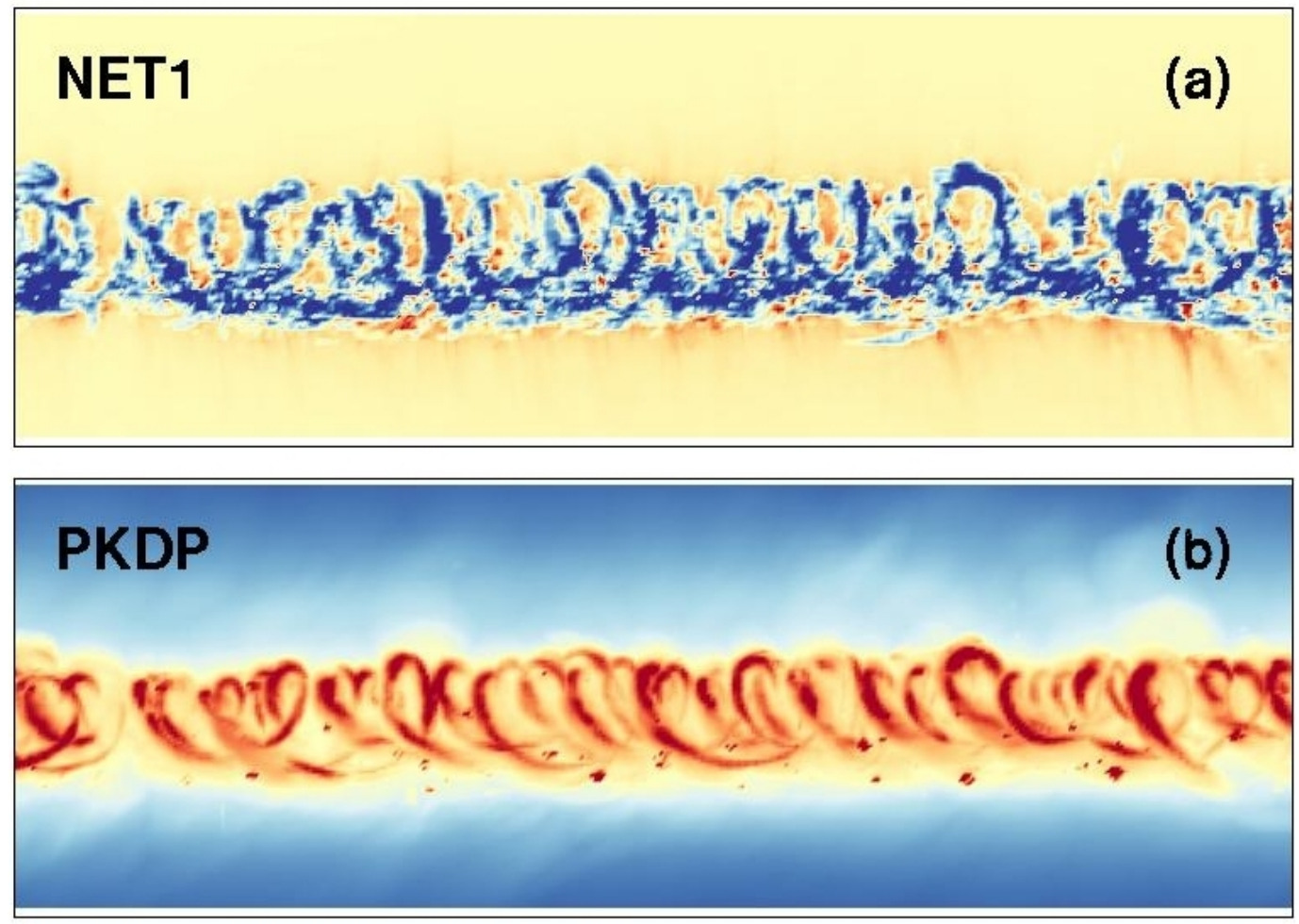

Figure 5.27: Surface tracks from a high-swirl tornado (run dmg2253) moving over a field of "heavy" debris $\left(S_{c}=12.4, A_{a}=1.9, A_{v}=1.5, A_{t}=0.36\right)$. (a) Deposition track, showing weak net removal coinciding with signatures of secondary vortices: (b) peak horizontal velocity at the first grid point above the surface and (c) peak pressure drop at the first grid slice above the surface. Deep blue in (a) represents a removal level of 0.0072 (when normalized to $R_{c}$ ), and deep red in (c) corresponds to a peak pressure drop of 1.25 (when normalized to $\left.\rho V_{c}^{2}\right)$. Lateral dimensions are $\pm 1.5 R_{c}$. 
and structure and motion of the near-surface vortex result, accompanied by dramatic changes in the surface markings. The figure illustrates both the sensitivity of tornado evolution to changes and asymmetries in the near-surface layer and the complexity and variability of the surface tracks that result.

Figure 5.29 shows sample instantaneous debris fluxes at the surface at late times in the respective cases of Figure 5.28. The most prominent features in the corner flow collapse cases typically not present in quasisteady cases are strong lines of deposition, or "lineation", occurring in regions of horizontal convergence situated away from the central vortex. It is important to note the possibility of such marks occurring in the field; without information about the location of the central vortex (i.e., through the presence of cycloidal marks or the availability of concurrent Doppler measurements), lineation outside the central flow could be mistaken for scalloping (c.f. Section 5.4). 

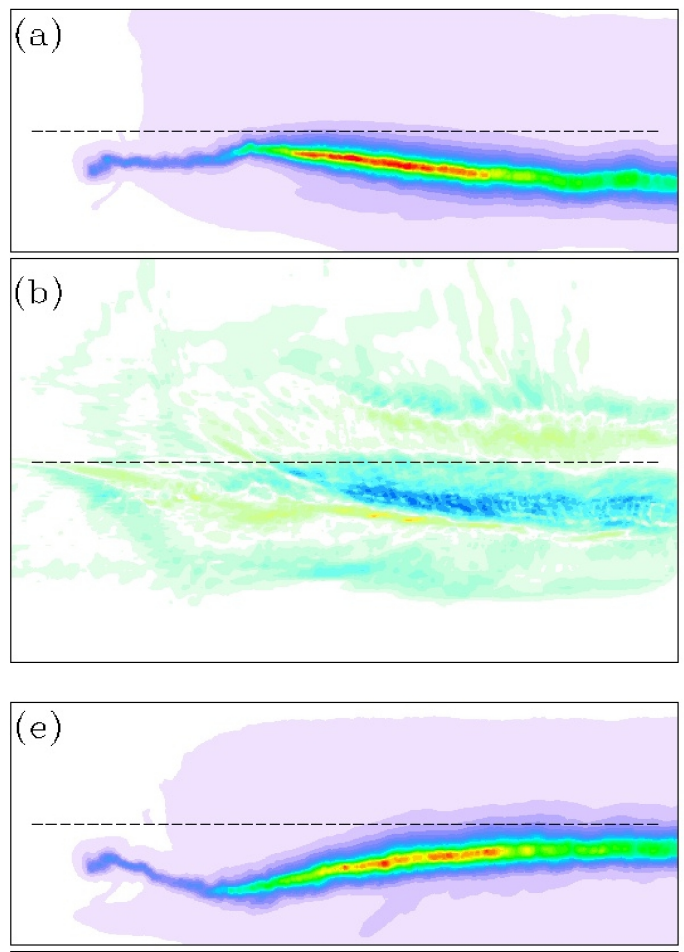

(f)

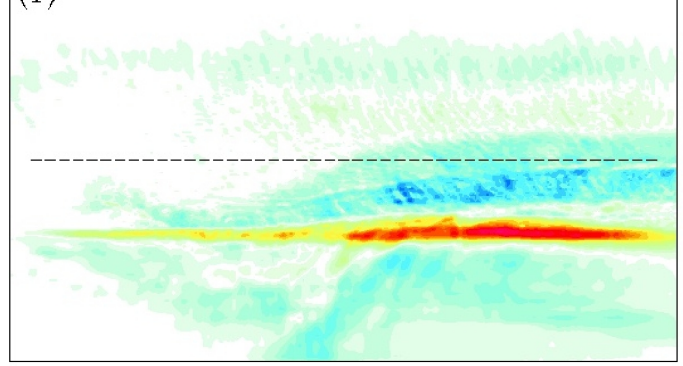

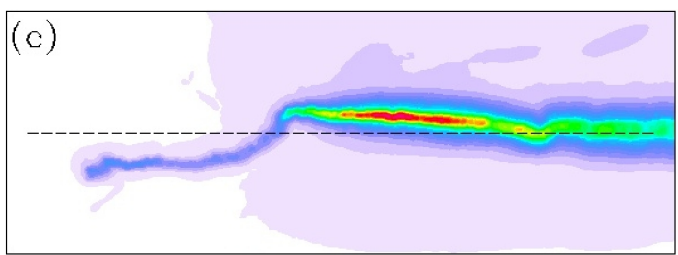
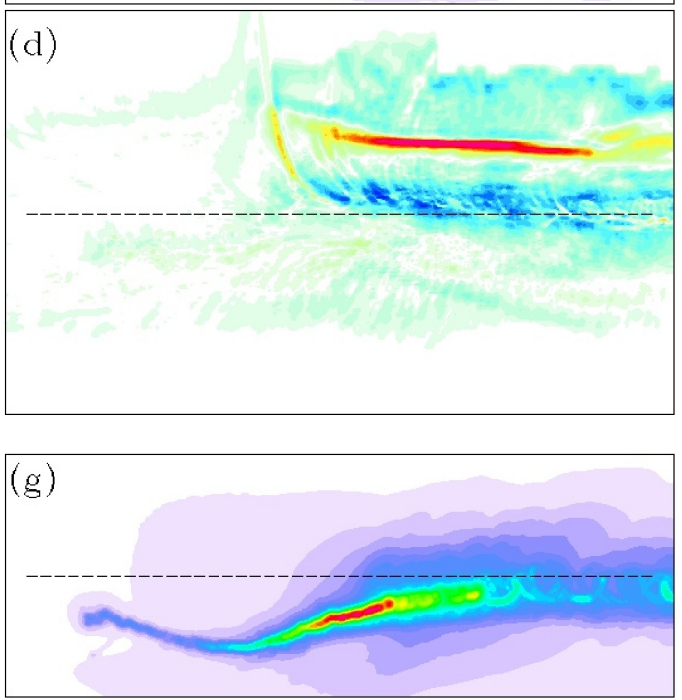

(h)

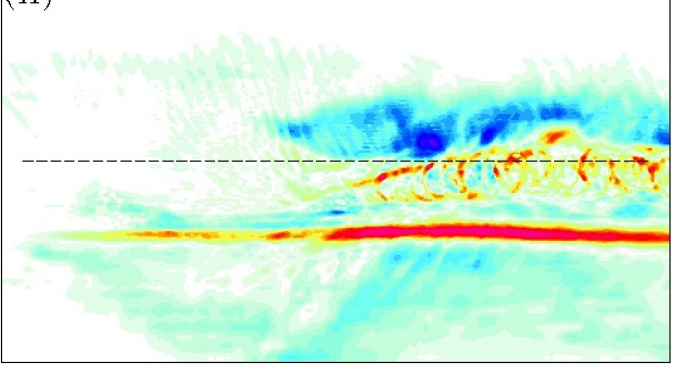

Figure 5.28: Surface tracks from four simulated corner flow collapse tornado evolutions showing peak pressure drops encountered at the surface (panels a,c,e,g) and net removal/deposition of $1 \mathrm{~mm}$ "sand" (panels b,d,f,h) with blue indicating heavy removal, red indicating heavy deposition. In each case the large-scale vortex aloft is translating from left to right (west to east) at $5 \mathrm{~m} / \mathrm{s}$ with the corner flow collapse triggered by impeding one quadrant of the low-swirl near-surface inflow: the northwest quadrant for panels a,b; NE for c,d; SW for e,f; and SE for g,h. The panel domain lengths are $1.65 \mathrm{~km}$ and the dashed lines indicate the track of the center of the large-scale vortex aloft. From (Lewellen and Zimmerman, 2008). 

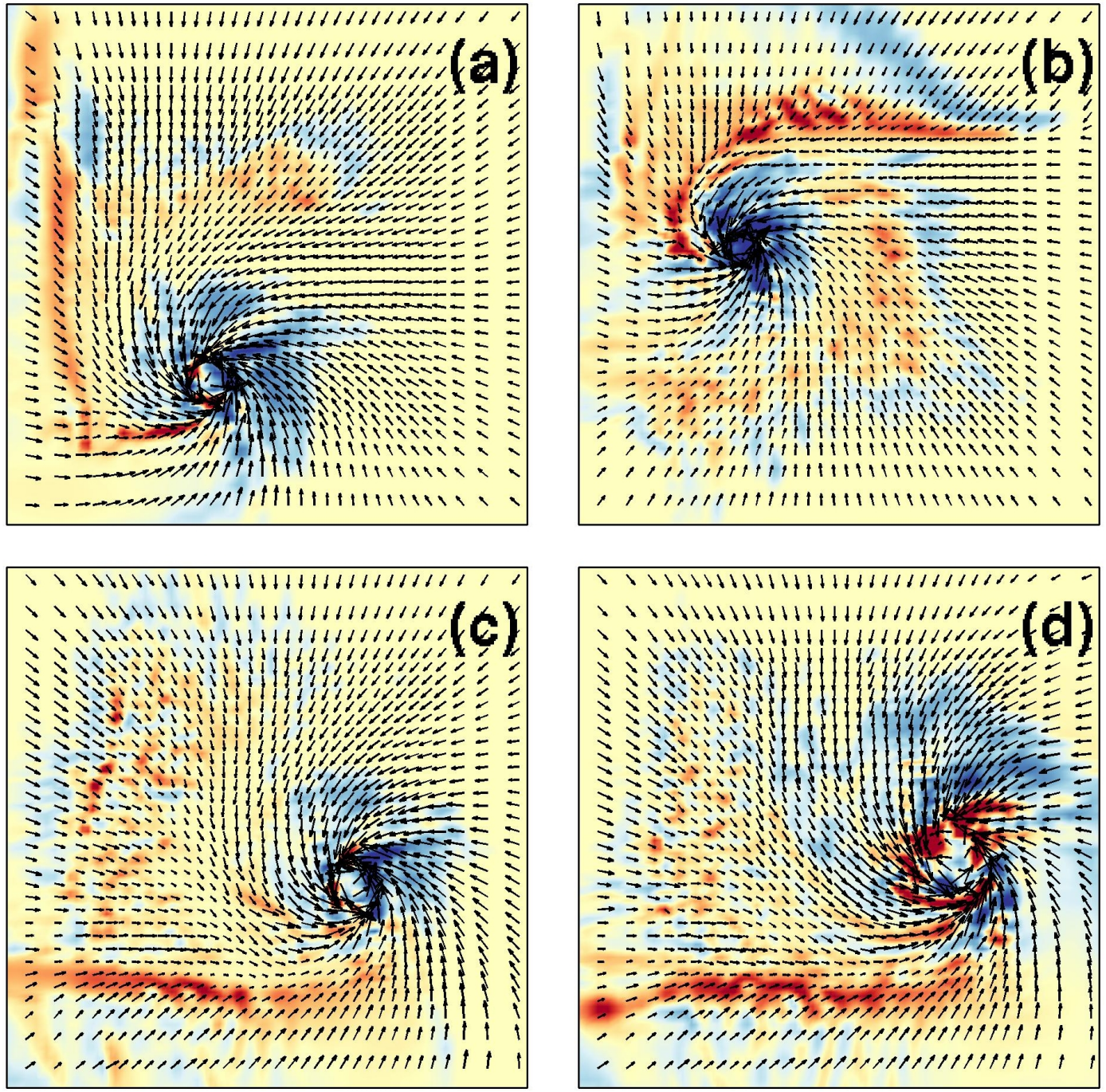

Figure 5.29: Sample instantaneous surface debris fluxes from the respective cases in Figure 5.28. Lateral dimensions are $600 \mathrm{~m}$. 


\section{Chapter 6}

\section{Quantitative analyses}

Attempts were made to quantitatively distill the very complex deposition signatures in simulated damage tracks down to simpler forms. The three categories of information pursued were surface mark shapes, spacings, and sizes. In this chapter, numerical efforts to extract these types of information from simulated tracks are described.

\subsection{Downstream averages to infer near-surface vortex radius and shift}

A "downstream average" is defined as the sum of a surface track quantity along the tornado's direction of travel; the quantity of interest here is net debris deposition unless otherwise noted. A handful of downstream averages for two sample collections of tornadoes are shown in Figure 6.1. Some downstream averages have already been shown in Figure 5.26.

Cases in which cycloidal marks are prominent typically have a double-peaked structure in the downstream profile; Figure 6.2 shows that in the selected cases these peaks correlate well with the extent of the deposition annulus at the surface. This radius, $R_{s}$, correlates with radii of the corner flow updraft annulus and maximum swirl velocity near the surface, potentially allowing inference of $S_{c}$ if the radial scale of the vortex aloft is known. A correlation between 
$S_{c}$ and $R_{s} / R_{c}$ was established in the top rows of Figures 5.3 and 5.5. Although debris effectively changes $S_{c}$ through its interactions with the airflow, it must be noted that $S_{c}$ calculated in the absence of debris is the quantity used in this work (c.f. discussion in Section 1.2).

The downstream peak data for cycloids, when available, can also be used to calculate the mean horizontal shift of the near-surface vortex with respect to the flow aloft. Vortex shift, $\delta_{s}$ is defined here as the difference between the center of the vortex aloft and the central location between the peaks normalized to the core radius $R_{c}$. Translation velocity was observed qualitatively to shift the vortex (e.g. the progression along the columns of Figure 5.9); however, the vortex shift is plotted against $A_{t}$ in Figure 6.3, showing no clear correlation. Decreasing swirl ratio was also observed to increase the vortex shift (e.g. the progression along the bottom row of Figure 5.3); the shift is plotted against $A_{t} S_{c}^{-1}$ in Figure 6.4 , revealing a correlation.

When attempting to infer tornado parameters from real surface tracks, concurrent Doppler scans aloft could provide information about $R_{c}, V_{c}, U_{\text {trans }}$, and the position of the vortex aloft. These quantities, in turn, provide direct estimates of $A_{t}$ and $A_{a}$. The parameter $A_{v}$ is less certain due to natural variability in surface debris availability conditions; a video record of debris cloud structure could aid in estimating $A_{v}$. An analysis of the track can perhaps be most informative when the underlying surface is relatively uniform but the track appearance evolves significantly, allowing some of the uncertainty in debris properties to drop out of the problem. The presence of cycloidal marks allows $\delta_{s}$ and $R_{s}$ to be inferred; the time-progressions of $\delta_{s} / R_{c}, R_{s} / R_{c}$, and $A_{t}$, (and changes in the qualitative collections of marks observed) could then provide quantitative evidence of the evolution of $S_{c}$ along the tornado's path. 


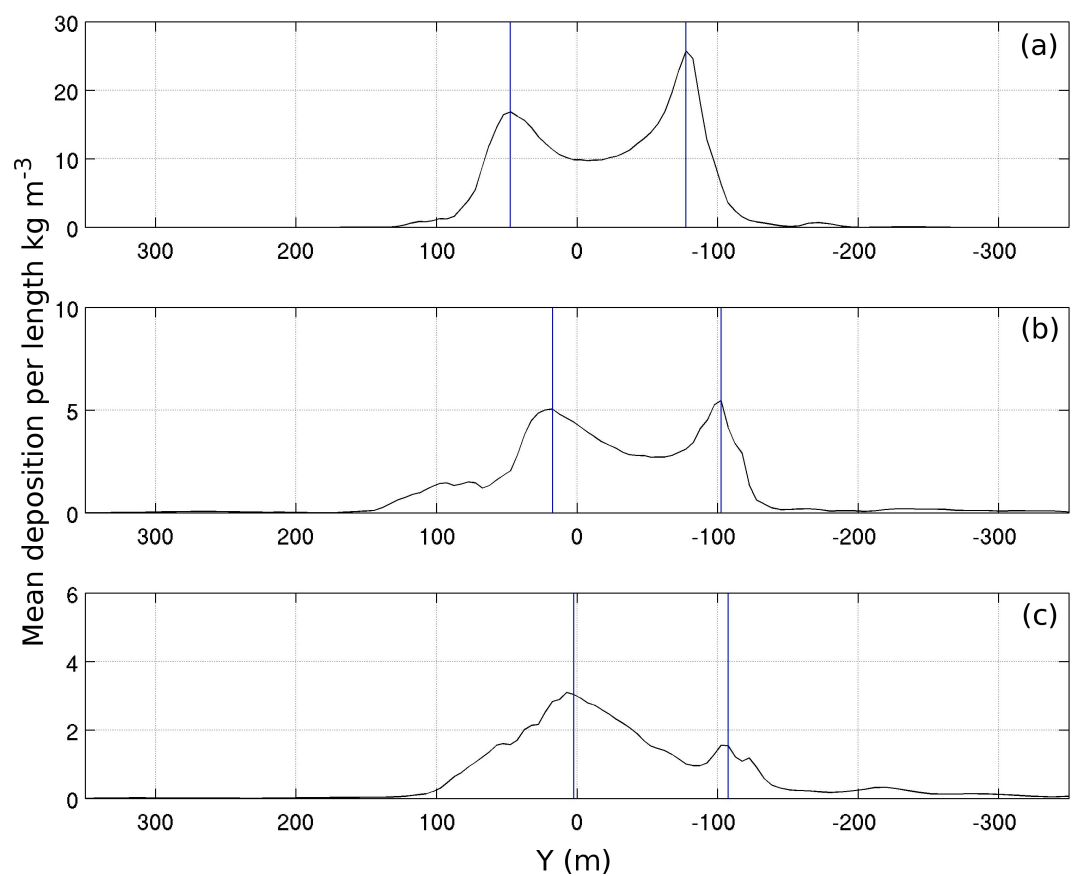

Figure 6.1: Downstream averages from a collection of high-swirl tornadoes moving at different speeds, showing locations of deposition peaks (blue lines). (a) $U_{\text {trans }}=5 \mathrm{~m} \mathrm{~s}^{-1}$ (dmg2059), (b) $U_{\text {trans }}=15 \mathrm{~m} \mathrm{~s}^{-1}$ (dmg2014), (c) $U_{\text {trans }}=25 \mathrm{~m} \mathrm{~s}^{-1}$ (dmg2060).

\subsection{Orientation histogram to capture surface mark shapes}

A new analysis technique was developed to capture the shapes of surface marks averaged over the collection in a track. This algorithm essentially uses image processing techniques to map tracks from the original "along-track vs. cross-track" $(x, y)$ representation to a "mark-angle vs. cross-track" $(\theta, y)$ representation and then back to $(x, y)$, giving a mean "fingerprint" of the shapes of the most prominent marks (e.g. fig. 6.5). The $(\theta, y)$ plot can be used directly to determine some quantities; e.g., the relative $y$ position of the crossing point of two branches in $(\theta, y)$ is found to correlate with the ratio of $U_{\text {trans }}$ and a near-surface swirl velocity.

In this method the surface track is treated as a heightfield, with the net deposition level serving as the height, $z_{\text {dep }}(x, y)$, above zero. $x$ is the spatial coordinate along the tornado's direction of travel and positive $y$ lies along an observer's left when standing on the ground and facing along the $x$-axis. $z_{\text {dep }}$ is correlated with the debris layer height above ground 

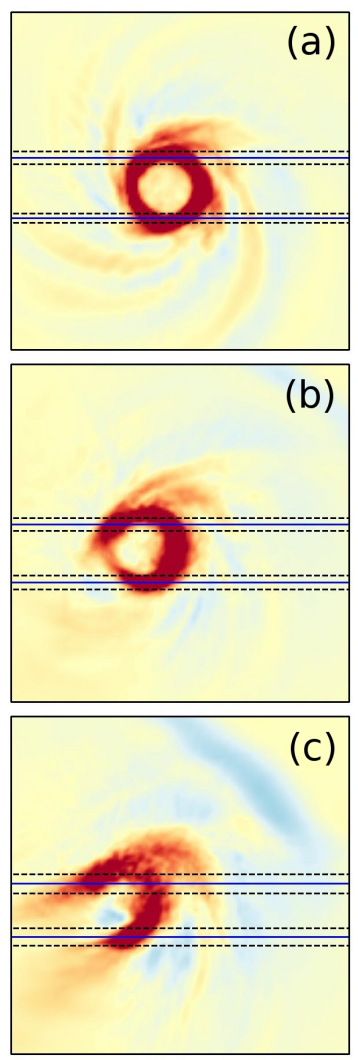

Figure 6.2: Time-averaged vertical surface debris flux with locations of deposition maxima (blue lines) and approximate half-width at half-max of each deposition peak (black lines) for the respective cases in Figure 6.1a-c. Dimensions span $\pm 350 \mathrm{~m}$. Respective differences between locations of the deposition peaks are approximately (a) $125 \mathrm{~m}$, (b) $120 \mathrm{~m}$, (c) 110 $\mathrm{m}$, giving a relatively uniform estimate of the size of the deposition annulus. 


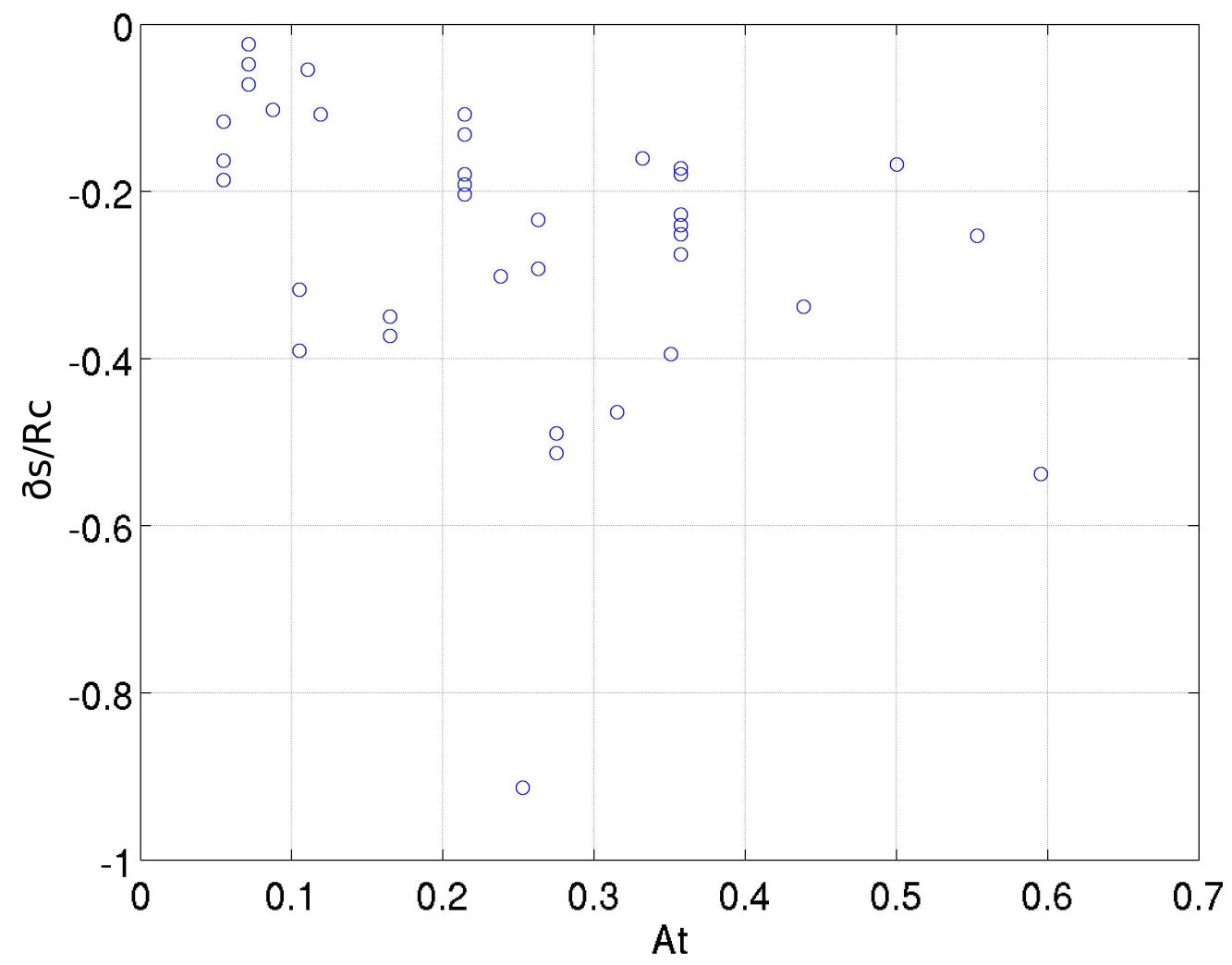

Figure 6.3: Vortex shift $\delta_{s}$ relative to $R_{C}$ aloft versus $A_{t}$.

level. Along the two-dimensional surface $z(x, y)$, net deposition swaths appear as ridges and net removal swaths appear as valleys, with the "sharpness" of each feature being determined by its height or depth and cross-ridge or cross-valley width. Narrow, deep (or high) features are considered sharp while wide, shallow features are considered diffuse.

Mathematically, sharp marks have large second derivative of $z(x, y)$ across their paths, taking on a negative value for marks of net deposition (e.g. Figure 6.6). The eigenvectors of the Hessian matrix of local second derivatives provide a convenient coordinate system in which to compute the sharpness (Lindeberg, 1996). The Hessian matrix is given by

$$
\left[\begin{array}{ll}
\partial_{x x} z_{d e p} & \partial_{x y} z_{d e p} \\
\partial_{y x} z_{d e p} & \partial_{y y} z_{d e p}
\end{array}\right] \equiv\left[\begin{array}{cc}
L_{x x} & L_{x y} \\
L_{y x} & L_{y y}
\end{array}\right]
$$




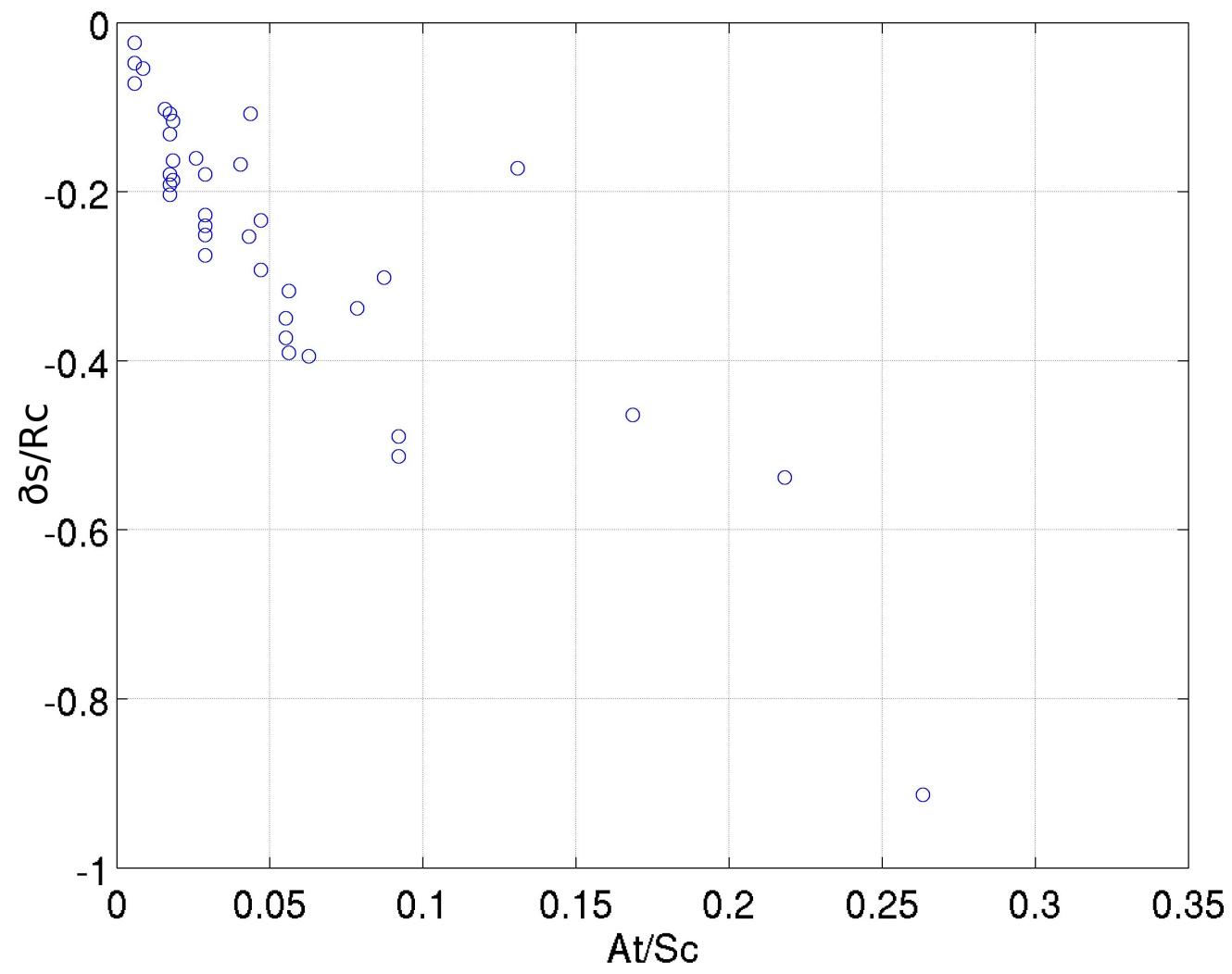

Figure 6.4: Vortex shift $\delta_{s}$ relative to $R_{C}$ aloft versus $A_{t} / S_{C}$. 
at each point $(x, y)$. The eigenvalues are

$$
\begin{gathered}
\eta_{1}=\frac{1}{2}\left(L_{x x}+L_{y y}\right) \\
\eta_{2}=\frac{1}{2} \sqrt{\left(L_{x x}-L_{y y}\right)^{2}+4 L_{x y}^{2}}
\end{gathered}
$$

The eigenvector pointing along a local ridge is that corresponding to the lowest of the two eigenvalues; one way to compute its angle with respect to the $x$-axis is

$$
\theta_{r}=\arctan \left(\frac{\eta_{\min }-L_{x x}}{L_{x y}}\right)
$$

Finally, the second derivative across a ridge or valley which provides a measure of sharpness is given by

$$
L_{u u}=\sin ^{2} \theta_{r} L_{x x}-2 \sin \theta_{r} \cos \theta_{r} L_{x y}+\cos ^{2} \theta_{r} L_{y y}
$$

The strongest, sharpest marks are typically associated with deposition: they have high contrast, defined as

$$
\delta I \equiv z_{d e p}\left|L_{u u}\right|
$$

where $L_{u u}<0$ for deposition swaths.

\subsubsection{Extracting the average "fingerprint" mark}

To distill geometric information about surface mark shapes from the standard $(x, y)$ track represetation into a simpler form, the contrast $\delta I$ is binned by $\theta_{r}(x, y)$ and $y$, effectively removing all dependence on $x$. The resulting "orientation histogram" captures the angles $\theta_{r}$ of the most prominent marks versus their locations in $y$ (e.g. middle column of Figure 6.5).

The position-angle curve can be transformed back to $(x, y)$-space to yield a mean spatial representation of the most prominent marks in a track (e.g. the right column of Figure 6.5). This is accomplished through the relation $d y / d x=\tan \theta_{r}$, which can be solved for $d x$ and 

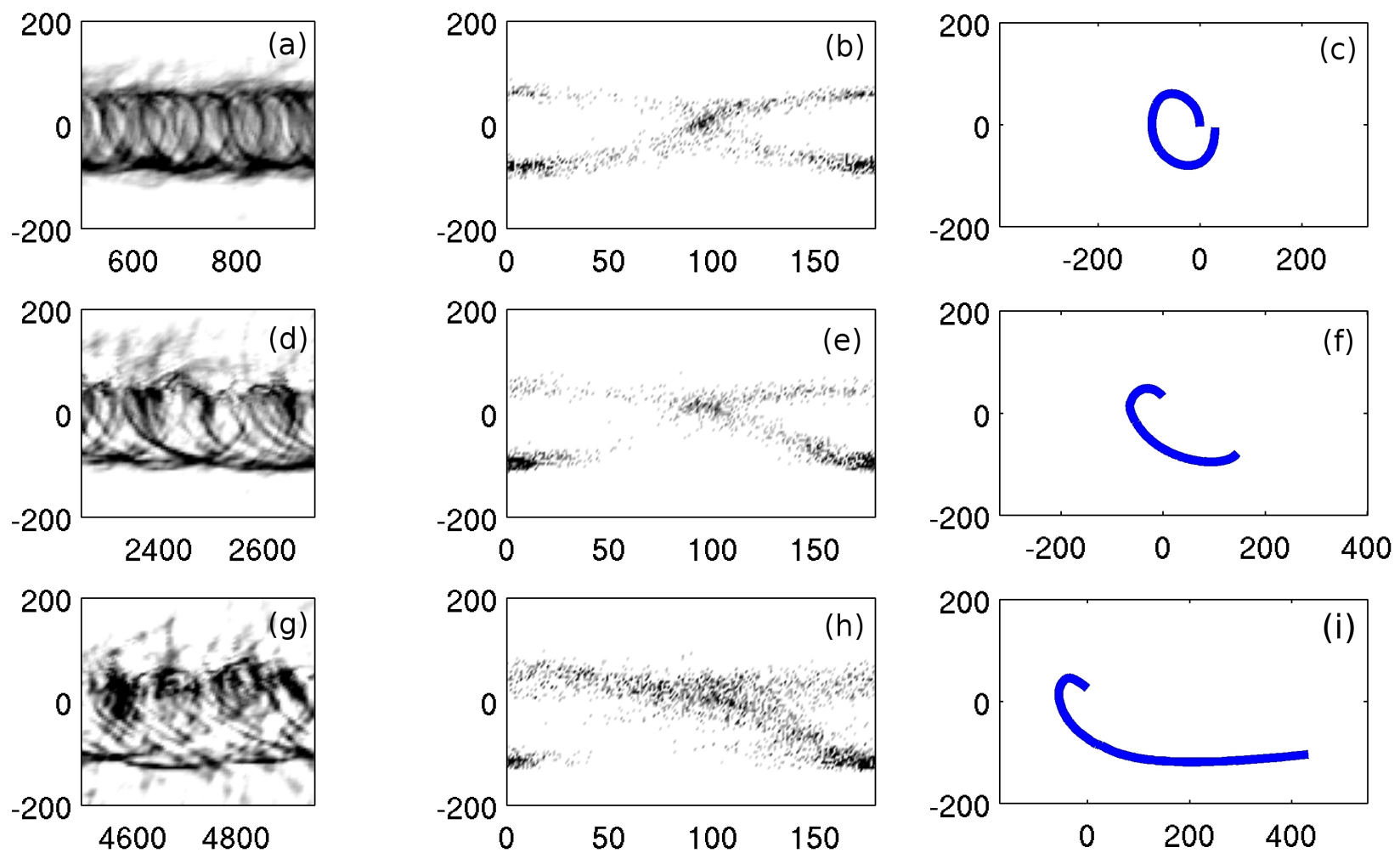

Figure 6.5: Left column: Deposition tracks for sample high-swirl runs. Middle column: "chromosome" patterns for the corresponding tracks in the left column. Right column: reconstructed mean surface marks for the chromosomes. Top to bottom: $U_{\text {trans }}=5 \mathrm{~m} \mathrm{~s}^{-1}$ (dmg2080), $15 \mathrm{~m} \mathrm{~s}^{-1}$ (dmg2081), $25 \mathrm{~m} \mathrm{~s}^{-1}$ (dmg2082).

integrated to give $x=\int d x=\int_{0}^{\theta} \tan ^{-1}(\phi) d y(\phi)$. There are, however, singularities in the integrand when $\tan \theta=0$; these are interpolated across prior to integration by assuming that $d x$ must be continuous. The resulting spatial $(x, y)$ curve gives an idea of the mean shape of the prominent marks. It is possible that reconstructed marks could be used to infer information about the near-surface flow.

\subsubsection{Extracting a near-surface swirl velocity scale}

Cycloidal marks typically have a double-branched "chromosome"-like character in $(y, \theta)$ space (e.g. middle column of Figure 6.5). It was found that the crossing point $y_{\text {cross }}$ of the chromosome branches with respect to the footpoints of the branches (i.e. the $y$-locations where the branches cross $\theta=(0, \pi))$ shifts with increasing $U_{\text {trans }}$. This trend is evident in 

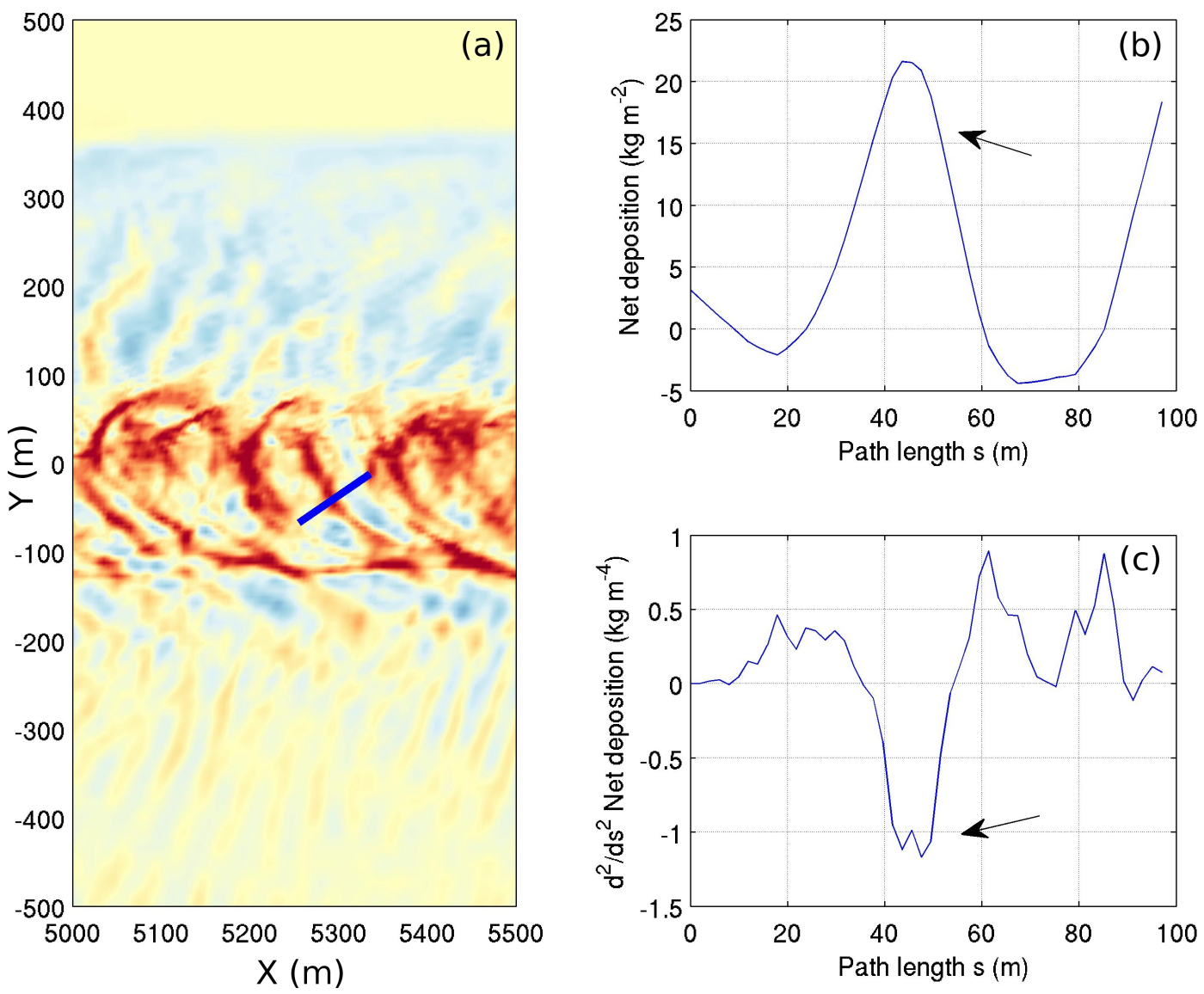

Figure 6.6: (a) Portion of the high-swirl track from run dmg2082, showing correlation between the second derivative of net deposition and local sharpness of a selected surface mark. Deep red/blue correspond to deposition/removal levels of magnitude $30 \mathrm{~kg} \mathrm{~m}^{-2}$. (b) Slice of net deposition along the path of the solid blue line in (a). (c) Second spatial derivative of the deposition profile in (b), showing negative peak where the local deposition mark is strongest. Black arrows roughly denote central location of the surface mark in question. 
the middle column of Figure 6.5.

To understand the shift, ideal cycloidal patterns were generated numerically and analyzed through the orientation histogram technique (e.g. Figure 6.7). It must be noted that it is unnecessary to assume that surface marks are cycloidal to get a usable correlation; however, ideal cycloids were studied for example and motivation. The ideal cycloid is essentially the path followed by a single point on a uniformly rotating, translating circle; the ideal cycloid centered on the line $y=0$ is given by

$$
\begin{gathered}
x(t)=U t+R \cos \left(\frac{V t}{R}\right) \\
y(t)=R \sin \left(\frac{V t}{R}\right)
\end{gathered}
$$

where $U$ is the translation velocity of the center of the circle, $V$ is the rotation velocity of the circle, and $R$ is the circle's radius (the phase of rotation has been chosen so that this cycloid passes through $x=R$ at $t=0$ and there is no extra phase term in the sinusoidal functions). For ideal cycloids the chromosome crossing point always occurs when the tangent angle to the cycloid curve is

$$
\theta_{\text {cross }} \equiv \arctan \left(\frac{d y}{d x}\right)_{\text {cross }}=\pi / 2
$$

Computing $d y / d x$ from the ideal cycloid equations and inserting it into the definition of $\theta_{\text {cross }}$ gives

$$
\tan \theta_{\text {cross }}=\frac{\sqrt{1-\Psi^{2}}}{\frac{U}{V}-\Psi}
$$

where $\Psi=y / R$. Since $\theta_{\text {cross }}=\pi / 2$, the condition $\Psi=U / V$ must hold at the crossing point; indeed, numerical tests on ideal cycloids support this result (e.g. Figure 6.8).

To actually extract the $(\theta, y)$ crossing point from cycloidal marks in damage tracks, an adaptive "snake" algorithm was developed (wherein a connected set of points is given freedom to follow gradients in the histogram's intensity in $(\theta, y))$. This algorithm was used to pick out the strongest line-like features in the histogram, capturing the branches as $n$ 
curves $y_{n}\left(\theta_{r}\right)$ on subintervals of $\theta_{r} \in[0, \pi]$. Due to noise in the histogram and the possibility of multiple basins of attraction for each branch this method requires smaller portions of the branches to be detected independently under user oversight and then stitched together and smoothed to yield a piecewise position-angle curve $y\left(\theta_{r}\right)$ on $\theta_{r} \in[0,2 \pi]$. Normalizing the crossing point location, $y_{\text {cross }}=y(\pi / 2)$ or $y(3 \pi / 2)$, to the locations of the branch footpoints, $y_{\max }=\max (y(0), y(\pi))$ and $\left.y_{\min }=\min (y(0), y(\pi))\right)$, yielded the normalized crossing point:

$$
\Psi_{\text {cross }}=\frac{y_{\text {cross }}-\left(y_{\max }+y_{\min }\right) / 2}{\left(y_{\max }-y_{\min }\right) / 2}
$$

Errorbars on measurement of $\Psi_{\text {cross }}$ are estimated for each run by averaging the differences between the raw and smoothed representations in $y\left(\theta_{r}\right)$, although this method often underestimates the spread in $\Psi_{\text {cross }}$ along a larger body of runs due to variations between runs. Standard deviations of up to $30 \%$ in $\Psi_{\text {cross }}$ have been observed to occur between different portions of the same track; however, $\Psi_{\text {cross }}$ tends to converge to a given value with increasing length of quasisteady track. Values of $\Psi_{\text {cross }}$ quoted in this work are those computed for the longest quasisteady portions of tracks available.

The crossing point correlates with $A_{t}$ for a collection of tornadoes with $3.0<S_{c}<$ 12.6 (e.g. Figure 6.9). This measure could be used in the field to estimate $V_{c}$ if $U_{\text {trans }}$ is known over a relatively quasisteady track of cycloids. It is worth noting that Fujita and colleagues previously developed a geometric approach that aimed to infer a near-surface swirl velocity scale from radii, spacings, and multiplicities of cycloidal marks (Fujita et al., 1970). However, Fujita and colleagues' approach was limited by uncertainties in the origins of cycloidal marks and required assumptions about how different marks were connected. The orientation histogram representation circumvents the need to connect sets of marks, capturing shape information without regard to how the marks are spaced, how many marks exist, or how uniform or intermittent segments of each mark are, thus providing a potentially useful substitute for the previous algorithms. 

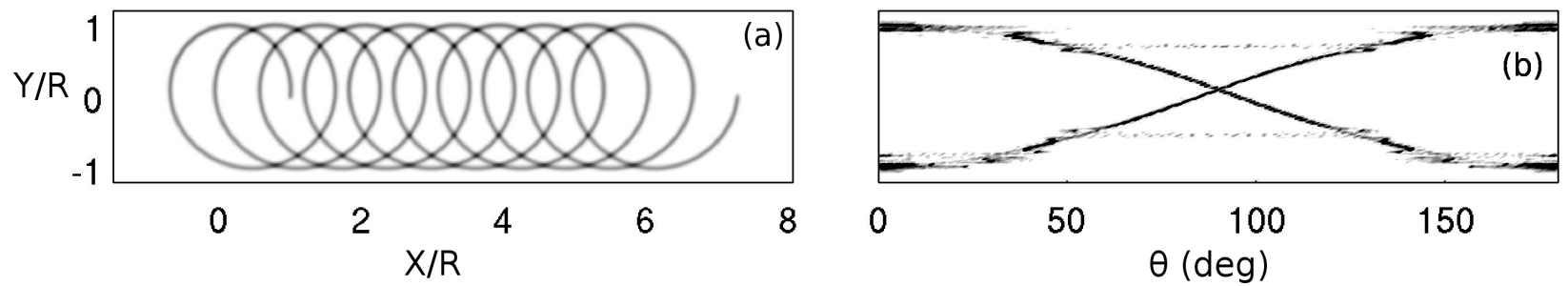

Figure 6.7: (a) Ideal cycloid with $\mathrm{U} / \mathrm{V}=1$. (b) Resulting "chromosome" pattern for the track in (a), with branch crossing point at $\Psi=0.1$.

To yield useful results, the method developed in the present work would require higherquality imagery than is currently available in the literature. In analogy with ideal cycloids, several candidate measures of a near-surface tornado velocity scale were tested for correlation with $U_{\text {trans }} / \Psi_{\text {cross }}$, including the maximum horizontal velocity at $z=0.5 \mathrm{~m}$ and the maximum swirl velocity in the corner flow. Neither yielded a good correlation (e.g. the attempted correlation with maximum horizontal velocity at $z=0.5 \mathrm{~m}$ is shown in Figure $6.10)$.

\subsection{Analyses to infer surface mark spacings}

Analyses were run on collections of damage tracks in attempts to extract mean spacings and multiplicities of surface marks without requiring information about their shapes. These included using hand tracing of marks, autocorrelations, fast fourier transforms, and wavelet transforms.

Sample results from hand tracing of marks are shown in Figure 6.11. The most intense pieces of individual marks were traced on a printout of each damage track, and the approximate integrated length of the traced lines was divided by the apparent radius of the marks to give a total number of marks $N$ per length of track $L$. While this method is highly subjective, it revealed a possible correlation between cycloidal spacings $(L / N)$ and translation velocity that encouraged further efforts to extract mark spacings.

Autocorrelations of net deposition along the direction of vortex translation have been 


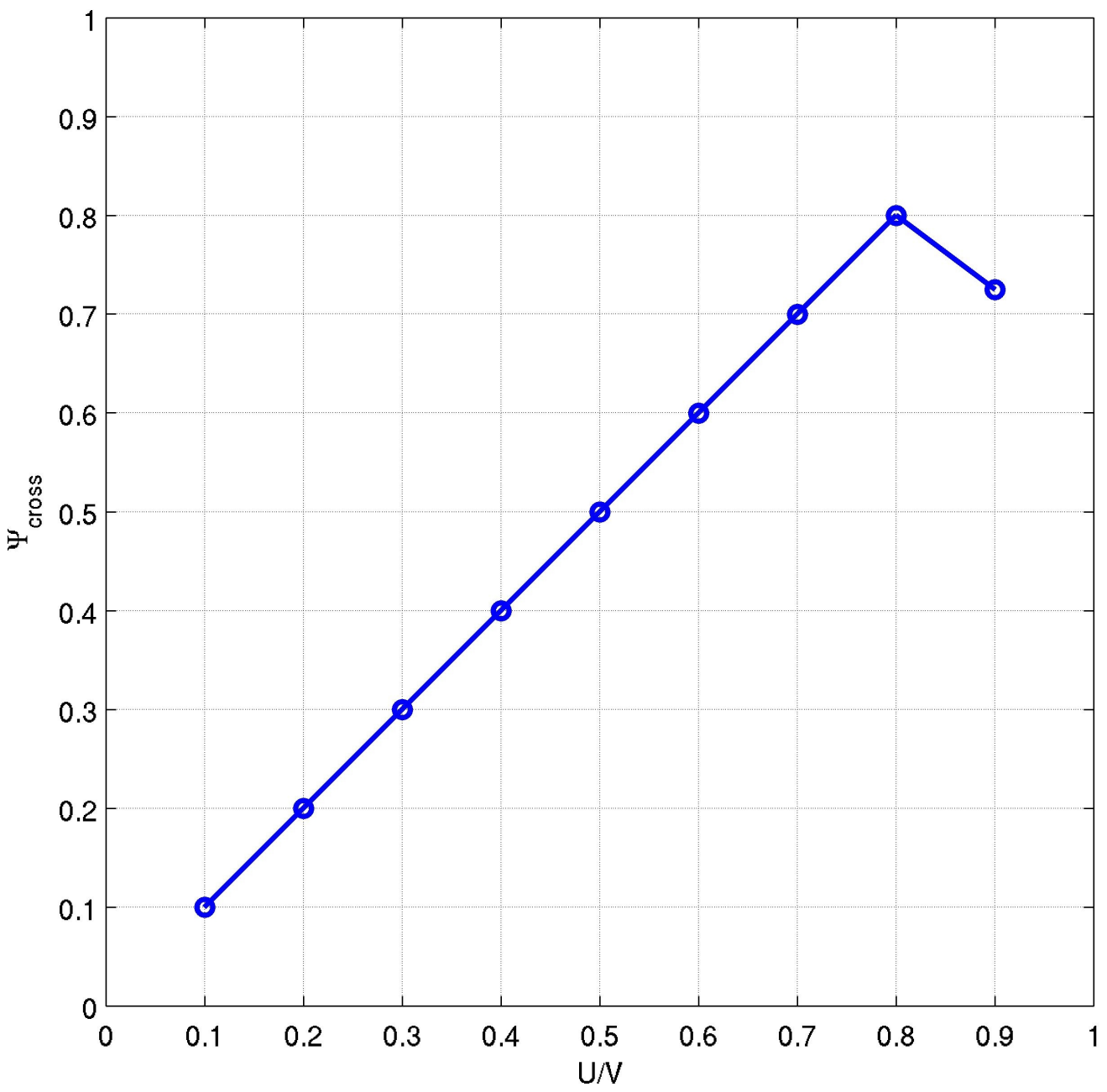

Figure 6.8: "Chromosome" branch crossing points from ideal cycloids versus U/V. The crossing point $\Psi_{\text {cross }}$ is equal to $U / V$, except for $U / V>0.8$ where the crossing point is difficult to distinguish numerically (it disappears entirely for $U / V>1.0$ ). 


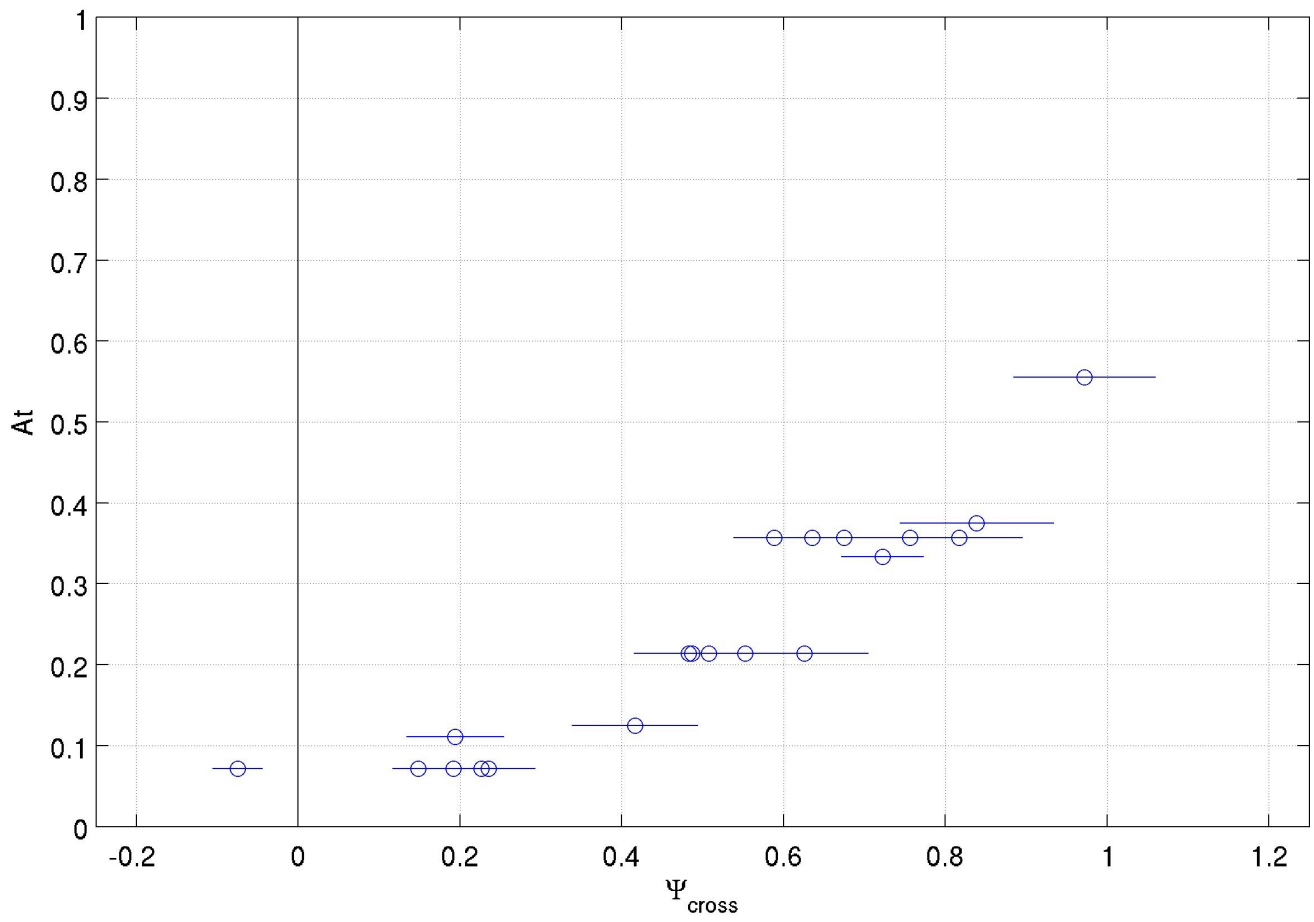

Figure 6.9: Dimensionless parameter $A_{t}$ plotted versus the crossing point of the two branches in $\left(\theta_{r}, y\right)$ for a group of simulated tornadoes that produced cycloidal marks. Errorbars calculated from the analysis method used are shown for each run; however, the effective error in measurement of $\Psi_{\text {cross }}$ when variations across runs are taken into account is roughly about \pm 0.1 on average. 


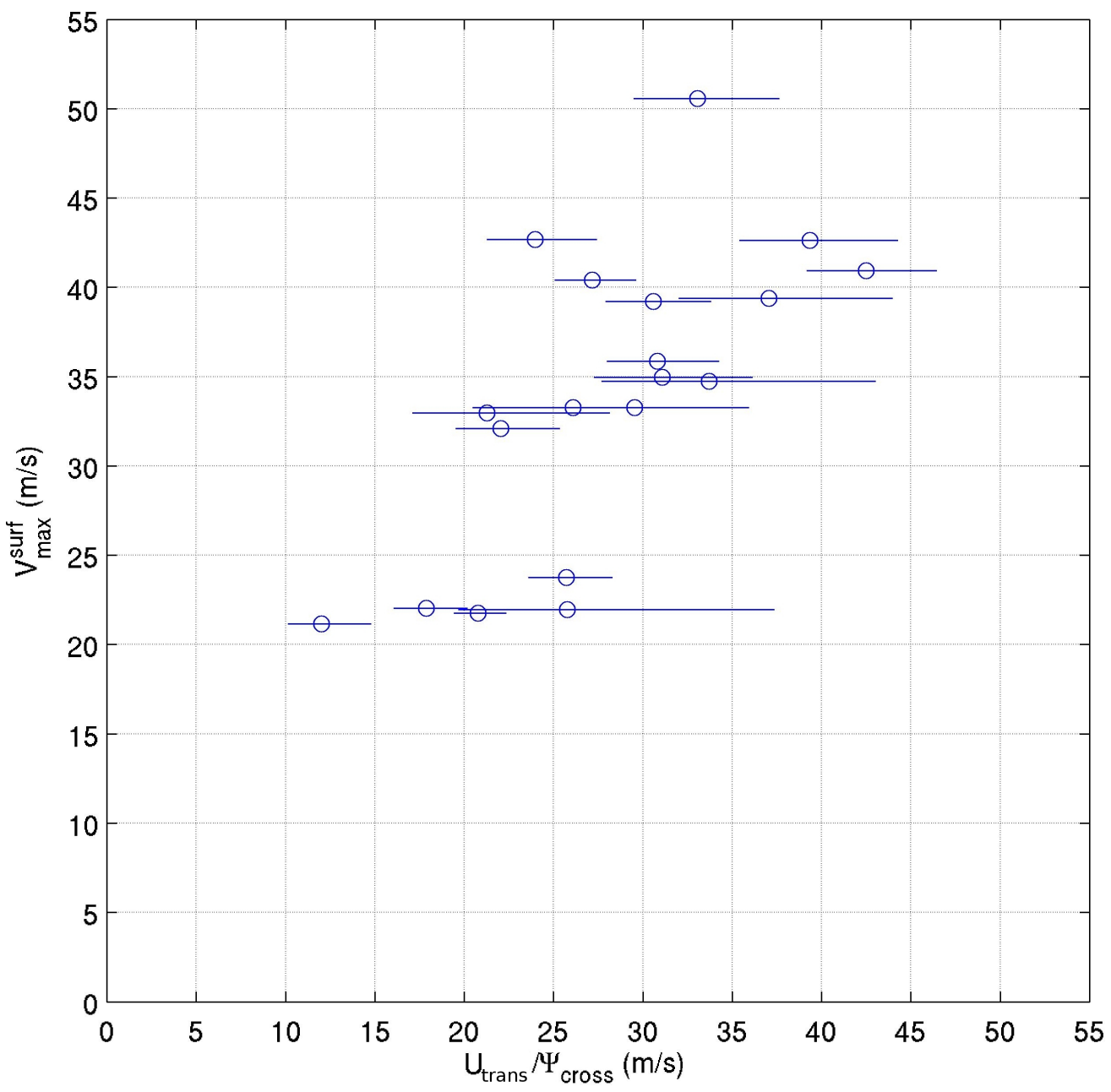

Figure 6.10: Maximum horizontal windspeed at $\mathrm{z}=0.5 \mathrm{~m}$ versus effective near-surface swirl velocity inferred from chromosome crossing point $\left(U / \Psi_{\text {cross }}\right)$ from a selection of cases with cycloidal marks. Errorbars calculated from the analysis method used are shown for each run; however, the effective error in measurement of $U_{\text {trans }} / \Psi_{\text {cross }}$ when variations across runs are taken into account is roughly about $\pm 10 \mathrm{~m} \mathrm{~s}^{-1}$ on average. 
largely unsuccessful in extracting mark spacings due to non-periodicity of the deposition processes responsible. The autocorrelation function is here defined as

$$
R(l, y)=\frac{\left\langle z_{d e p}(x, y)-\left\langle z_{d e p}(x, y)\right\rangle\right)\left(z_{d e p}(x-l, y)-\left\langle z_{d e p}(x, y)\right\rangle\right\rangle}{\left\langle\left(z_{d e p}(x, y)-\left\langle z_{d e p}(x, y)\right\rangle\right)^{2}\right\rangle}
$$

where the angle brackets indicate an average has been taken along $x$. Stacking the autocorrelation function along a series of adjacent parallel slices yields an effective transformation of the entire track from $(x, y)$-space into $(l, y)$-space, where $l$ is the autocorrelation lag. Sample results are shown in Figures 6.12 and 6.13. Except in select cases, the unsteady nature of the tracks typically smeared the autocorrelation function in lag-space, providing no clear indication of a primary spacing between marks. The utility of the "image" autocorrelation, which is essentially the integral of $R(l, y)$ along $y$, and the Fourier transforms of various slices and subimages were also explored to little effect.

The one-dimensional wavelet transform was explored for individual slices of deposition tracks along the direction of tornado travel. While it was able to resolve spacings between neighboring marks, the wavelet transform actually expanded the parameter space from $\left(x, y_{0}, z_{d e p}\right)$ to $\left(x, y_{0}, z_{d e p}, \lambda\right)$ where $y_{0}$ is the constant $y$-location at which the slice is taken and $\lambda$ is the local length scale of deposition features. This is contrary to the goals of analysis, which are primarily to distill the information available in surface tracks into a smaller set of descriptive parameters. $\lambda$ spectra were explored in an effort toward distillation (i.e. the signal in $\left(x, y_{0}, z_{\text {dep }}, \lambda\right)$-space was integrated along $\left.x\right)$; however, the $\lambda$-spectra did not exhibit sharply-defined peaks that would indicate a dominant spacing of any particular marks.

Currently, there is no effective numerical measure of surface mark spacing, due primarily to the marks' complicated, two-dimensional signatures and surface track turbulent variability. 


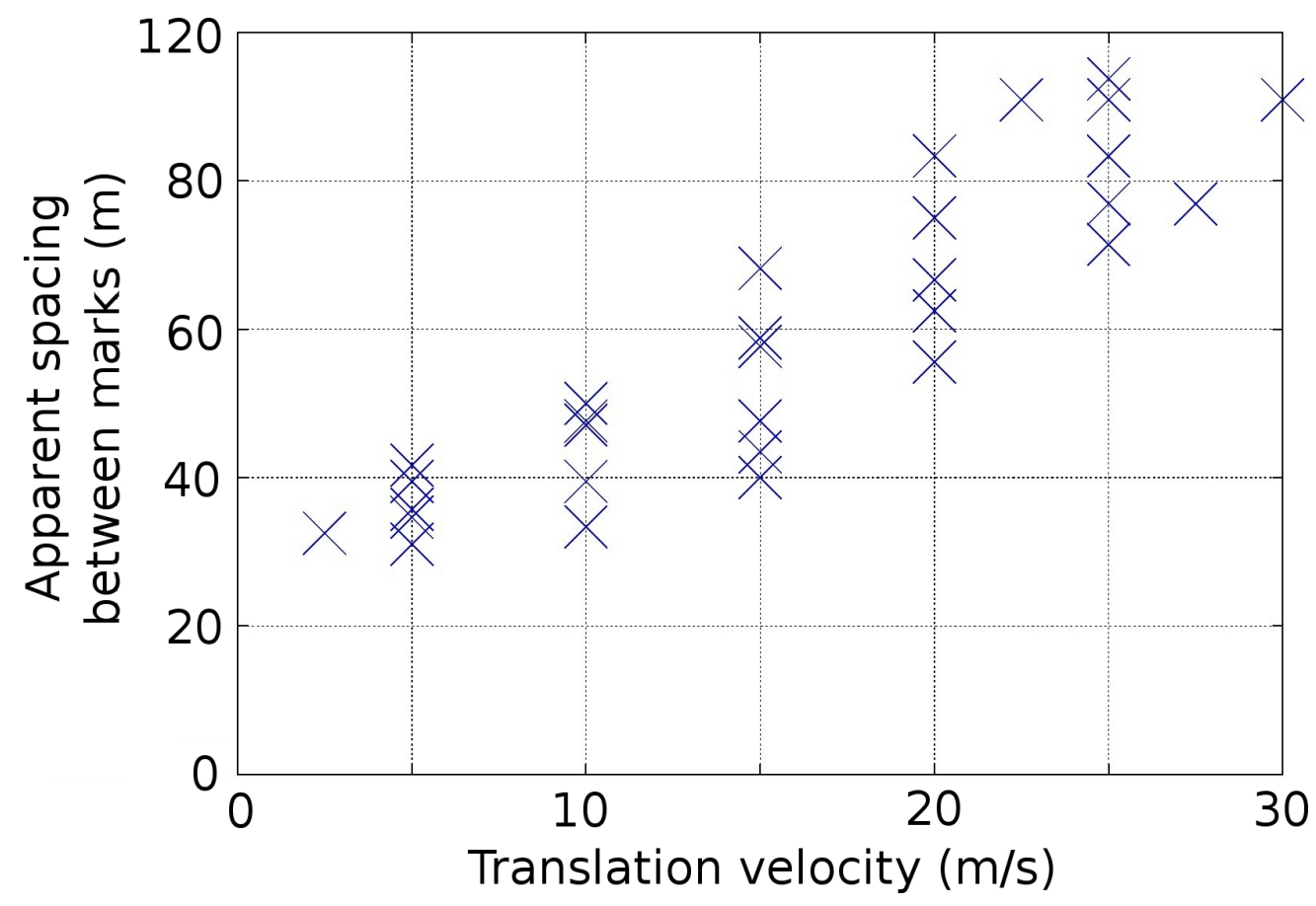

Figure 6.11: Surface mark spacings inferred by hand-tracing cycloidal marks in a collection of high-swirl tornadoes with $S_{c}=12.4, A_{v}=12.2, A_{a}=2.39$, and varying $A_{t}$. 

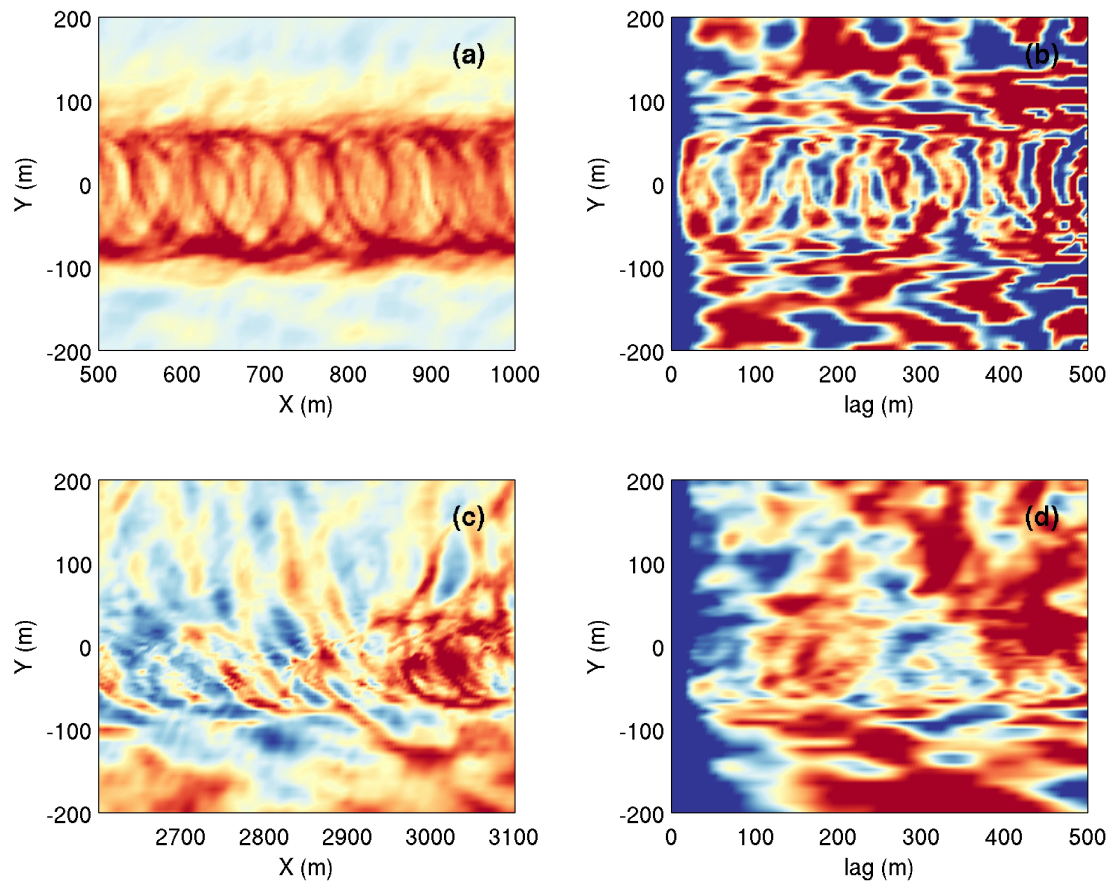

Figure 6.12: Autocorrelation along the tornado direction of travel for two selected deposition tracks: (a,b) high-swirl case (run dmg2080); (c,d) medium-swirl case dmg2011. In the righthand column deep blue and red correspond respectively to autocorrelation magnitudes of $+/-0.3$. 

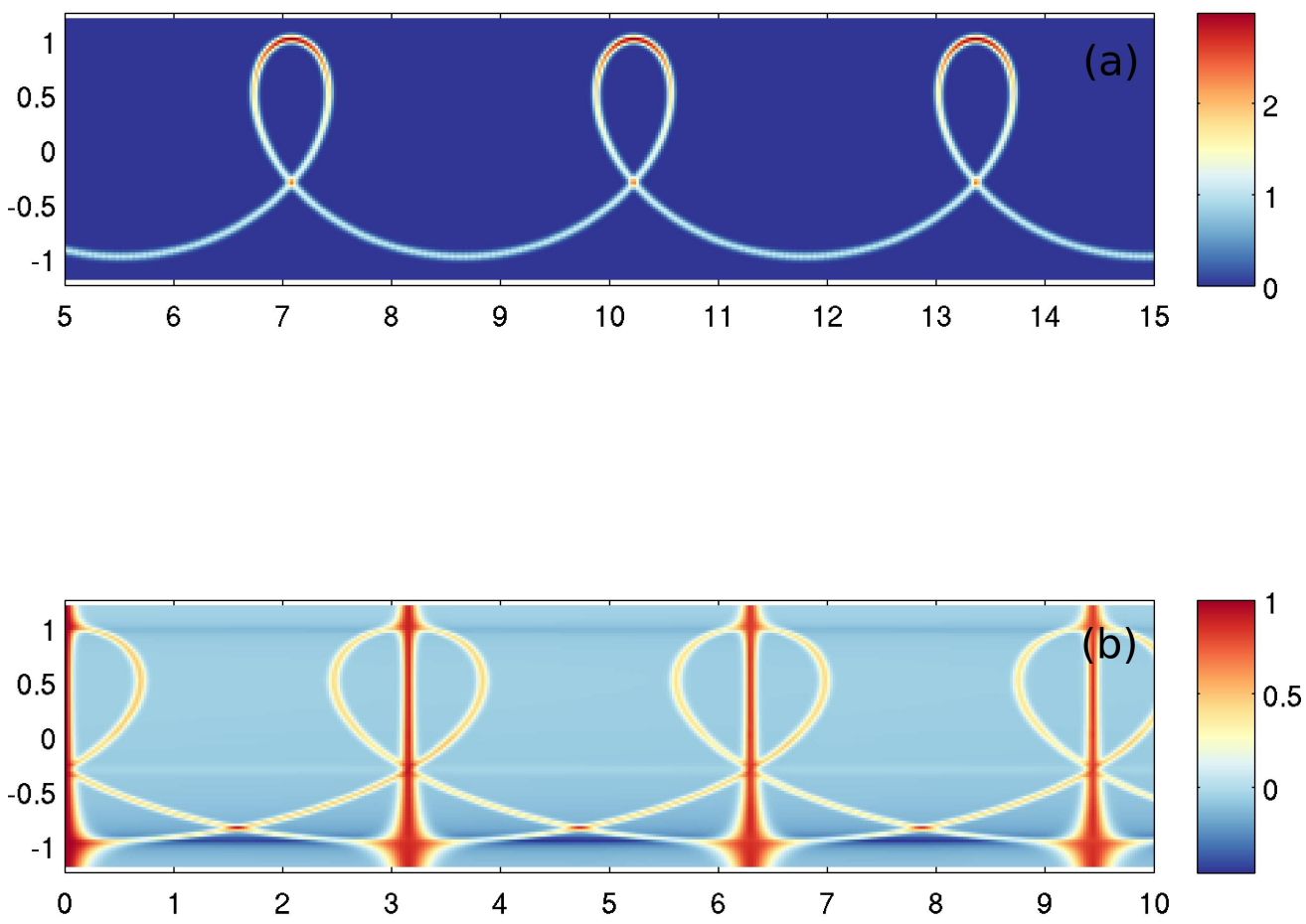

Figure 6.13: (a) Ideal cycloid with $\mathrm{U} / \mathrm{V}=0.5$. (b) Autocorrelation of the cycloidal signal in (a) along its direction of travel. 


\section{Chapter 7}

\section{Conclusions and discussion}

A large matrix of large-eddy simulations of tornadoes with debris was generated with three primary goals in mind: to investigate the physical origins of surface debris marks and compare them with historical interpretations; to study the variations of surface marks with tornado structure and intensity, debris type, and translation velocity; and, to learn what can be inferred about tornado structure from aerial photography of surface tracks in the field (possibly with additional information about the flow aloft). The simulation code employed was state of the art, including an accounting of where debris was removed and deposited on the surface to produce simulated surface tracks up to tens of kilometers long. Fujita and colleagues' original idea of inferring near-surface tornado wind velocities from the properties of surface marks was resurrected; previous difficulties in interpreting the physical origins of marks were circumvented by simulating the most important features of the complex nearsurface flow. This work also has direct relevance to dust devils that occur on Earth and Mars where loose debris is plentiful at the ground.

The majority of simulation runs included only one species of debris. Sensitivities to debris type were performed, showing that simulations with the same combination $\sigma d$ (where $\sigma$ is the debris particle mass density and $d$ is particle diameter) generally produced similar tracks, enabling extrapolation of results for sand-like debris to species of much lower density 
and higher diameter (e.g. dried corn stubble). In the presence of multiple debris species, deposition tracks of the individual species were nearly identical to one another with the exeception of modest variations in relative intensities of some marks. Also, the total deposition track (i.e. the sum of the individual tracks) in multispecies cases was generally found to be similar to the single-species surface track for some effective choice of debris $\sigma d$.

The greatest uncertainties in this work are in the parameterization of the surface. No particular real surface has been modeled; however, surface parameters were varied as tests of sensitivity. Surface marks were largely insensitive to changes in surface parameters that did not dramatically alter debris mass loadings or the balance between near-surface swirl and inflow levels. These variations are not captured entirely by the four dimensionless parameters considered in this work; in particular, the swirl ratio and spatial and velocity scales of normalization are computed in the absence of debris, requiring discussion in terms of an "effective" swirl ratio. Development of an alternative measure of swirl ratio including such effects as limited debris availability and debris-induced changes in the near-surface ratio of swirl to inflow would provide a more robust means of collecting the physics governed by many surface parameters into a smaller handful of variables. Also, variations of marks in more "exotic" situations where the near-surface inflow is impeded in different locations have been noted but not explored in depth.

A detailed taxonomy of simulated surface marks was compiled and their physical origins uncovered. Much of the historical nomenclature was robust enough to be retained even in light of simulation results that contradicted previous intepretations. The revised taxonomy of deposition marks for quasisteady simulations in the present work included cycloidal marks, scalloping, trailing arcs, and diffuse far-field swaths, with lineation appearing in a small handful of cases with time-varying boundary flow conditions.

The sharpest marks came from debris deposition; removal signatures were generally more diffuse. The strongest central deposition marks were from debris that could not follow the rapid corner flow upturn and was redeposited in an annulus underneath the central 
vortex (often taking the form of cycloids or arcs). Diffuse far-field swaths of deposition away from the central vortex were attributed to debris that had been fully lofted and centrifuged outward. Alternating patterns of removal and deposition further from the main vortex (trailing arcs) were attributed to debris-laden surface rolls in the inflow layer. Scalloping occured underneath the central vortex when the debris availability at the surface was limited or behind the vortex when trailing arcs aligned with the translation velocity vector. Lineation occurred further to either side of the central vortex in "corner flow collapse" cases where the inflow component was impeded in various quadrants of the near-surface flow. These marks could be confused with scalloping in the field if the location of the central vortex is not known (through the presence of cycloidal marks or the availability of concurrent Doppler measurements).

These findings contrast with some of those of Fujita and colleagues (Agee et al., 1975, 1977; Davies-Jones et al., 1978; Forbes and Wakimoto, 1982; Fujita, 1966, 1967, 1981; Fujita et al., 1967, 1970, 1976a,b), who postulated that secondary vortices were the primary mechanism responsible for gathering debris into the sharp marks observed in the field. No direct correlation was found between the paths of simulated secondary vortices and prominent deposition marks; furthermore, some of the types of marks previously attributed to secondaries were observed in simulated tornadoes where there was only a single vortex on the surface. Nonetheless, this study supports the more general conclusion of Fujita and colleagues that surface marks provide useful information about tornado structure and dynamics.

The qualitative appearance of surface marks was investigated in terms of the following dimensionless parameters:

- Corner flow swirl ratio $S_{C}$ (categorizing corner flow structure) (Lewellen et al., 2000)

- Acceleration ratio $A_{a}=V_{C}^{2} / g R_{C}$ (importance of centrifugal forcing relative to gravitational forcing) (Lewellen et al., 2008)

- Velocity ratio $A_{v}=V_{C} / w_{t}$ (relative ease of lofting) (Lewellen et al., 2008) 
- Translation velocity ratio $A_{t}=U_{\text {trans }} / V_{C}$ (importance of translation relative to swirl)

where $V_{c}$ and $R_{c}$ are the upper core velocity and radius scales, $g$ is gravitational acceleration, $w_{t}$ is the debris terminal velocity in freefall, and $U_{\text {trans }}$ is the surface-relative translation velocity of the vortex. Casting results in terms of these dimensionless parameters allows them to be more easily generalized to a wide range of vortices (e.g. to constrain the properties of vortices in the near-surface Martian environment). The range of parameters explored was roughly $0<S_{c}<13,1.5<A_{v}<30,0.5<A_{a}<77,0.05<A_{t}<0.6$.

Very-low-swirl tornadoes $\left(S_{c}<1.4\right)$ were not found to produce strong deposition marks because their surface wind levels were generally too low to remove debris. Otherwise, the swirl ratio was found primarily to capture the size of cycloidal marks relative to the size of the vortex aloft. The shift of the vortex center on the ground relative to its position aloft scaled with $A_{t} / S_{c}$ Raising $A_{t}$ increased the left-right asymmetry of cycloidal marks and the relative intensity of trailing arcs (e.g with respect to the other types of marks). Increasing $A_{v}$ caused far-field diffuse swaths to become more intense. Raising $A_{a}$ favored trailing arcs and increased the size of cycloidal marks through the effects of debris centrifuging.

Quantitative analysis methods were developed to distill surface track signatures into simpler forms capturing shapes, sizes, and spacings of marks. The downstream average of net deposition was often useful in extracting a characteristic near-surface vortex radius when cycloids were present. A rough correlation was found between $R_{s} / R_{c}$ and $S_{c}$ for tornadoes with cycloidal marks, where $R_{s}$ is the cycloidal radius, although there is considerable variability with $A_{a}$ and $A_{v}$. A variety of methods were explored for inferring surface marks spacings including autocorrelations and wavelet analyses.

An algorithm was developed to determine mean surface mark shapes using image processing techniques. This approach involved transforming surface tracks from the standard $(x, y)$-representation to a mark sharpness versus lateral position $(\theta, y)$-representation. The resulting "orientation histograms" often had single- or double-branched structure in $(\theta, y)$ which could be extracted for analysis. In the case of ideal cycloids, the crossing point of 
the branches correlates with the ratio of cycloidal translation to rotation $U_{\text {trans }} / V_{c}$, thus providing a potentially useful substitute for the algorithms of Fujita and colleagues (Fujita et al., 1970). Prospects for using the crossing point analysis to infer an effective near-surface $U / V$ ratio from cycloidal surface marks were explored.

At this time, the greatest need of the present work is a set of quantitative measures of the relative intensities of marks (to establish sharper correlations between populations of marks and the dimensionless parameters). The algorithms developed are a first step toward such measures; however, it is an extremely complex image processing task to detect and extract individual, overlapping marks of different intensities and widths. Also, the image-processing techniques developed in the present work rely heavily on user input and guidance. A scalespace approach (Lindeberg, 1996) in which continuous marks of different widths are detected based on local quantitative measures of sharpness could potentially circumvent these issues by providing a more robust, automatic analysis procedure.

It was hoped at the outset that many complications would drop out when interpreting the simulation set, enabling compilation of a simplified dictionary of surface marks and the ranges of conditions under which they occur. The deposition tracks of Figures 5.3, 5.5, 5.9, and 5.7 show that all four of the primary dimensionless parameters are important in governing the formation of surface marks. However, analysis of real surface marks in terms of dimensionless parameters should still be possible under fortuitous conditions in the field.

With concurrent information about the flow aloft, analysis of surface tracks could provide critical information about near-surface vortex structure currently unavailable to Doppler radar or in-situ probes alone. Consider the case in which a tornado has left surface marks in a relatively uniform field of debris (such that $w_{t}$ can be assumed relatively uniform over time) and there are concurrent Doppler radar measurements of the flow aloft. The translation velocity $U_{\text {trans }}$ and properties of the vortex aloft $\left(R_{c}\right.$ and $\left.V_{c}\right)$ can be estimated from properties of the peak velocity couplet in the Doppler data aloft, providing measures of $A_{t}, A_{a}$, and $A_{v}$ over time. With these variations known, the evolution of $S_{c}$ could be inferred from the quali- 
tative appearance of marks along different sections of the track. The prospects are promising enough to warrant documentation of surface marks in the field (likely through unmanned aerial vehicles), particularly when concurrent Doppler measurements are available. 


\section{Appendix A}

\section{Tables of runs}

Stationary runs without debris are listed in Table A.1, and were used as initial conditions for the debris-laden runs listed in Tables A.2-A.6. Default surface parameters for debris-laden runs are $c_{a}=0.12, c_{d}=0, c_{b}=1, c_{t}=0.5, c_{u}=1$, unless otherwise noted in the entries in this Appendix. 


\begin{tabular}{|c|cccc|c|cccc|}
\hline Name & $\begin{array}{c}R_{c} \\
(m)\end{array}$ & $\begin{array}{c}V_{c} \\
\left(\mathrm{~m} \mathrm{~s}^{-1}\right)\end{array}$ & $\begin{array}{c}R_{l} \\
(m)\end{array}$ & $\begin{array}{c}V_{l} \\
\left(\mathrm{~m} \mathrm{~s}^{-1}\right)\end{array}$ & $S_{c}$ & $\begin{array}{c}\omega \\
\left(\mathrm{s}^{-1}\right)\end{array}$ & $\begin{array}{c}\Gamma_{\infty} \\
\left(\times 10^{4}\right. \\
\left.\mathrm{kg} \mathrm{m}^{2} \mathrm{~s}^{-1}\right)\end{array}$ & $\begin{array}{c}R_{\text {in }} \\
(\mathrm{m})\end{array}$ & $\begin{array}{c}W_{\text {in }} \\
\left(\mathrm{m} \mathrm{s}^{-1}\right)\end{array}$ \\
\hline run3320-tav* & 102.4 & 47.6 & 72.4 & 67.3 & 1.9 & 0.0125 & 0.5 & $\mathrm{n} / \mathrm{a}$ & $\mathrm{n} / \mathrm{a}$ \\
run3300-tav* & 107.2 & 90.9 & 79.6 & 122.4 & 3.0 & 0.0125 & 1.0 & $\mathrm{n} / \mathrm{a}$ & $\mathrm{n} / \mathrm{a}$ \\
run3240-tav & 171.0 & 57.0 & 117.9 & 82.6 & 5.6 & 0.0100 & 1.0 & 50 & -12.5 \\
run2057-tav & 208.8 & 70.0 & 161.5 & 90.4 & 12.4 & 0.0150 & 1.5 & 100 & -12.5 \\
\hline
\end{tabular}

Table A.1: A subset of stationary runs without debris used as initial conditions for translating, debris-laden cases. $R_{c}$ and $V_{c}$ are characteristic spatial and velocity scales of the vortex aloft, $R_{l}$ and $V_{l}$ play the same respective roles in the corner flow, and $S_{c}$ is the corner flow swirl ratio. The values of these parameters result from the user-defined values of the constant horizontal convergence at the boundaries $\omega$, the far-field angular momentum $\Gamma_{\infty}$, and downflow velocity $W_{i n}$ imposed at the top boundary within a disk of radius $R_{i n}$. Runs marked with ${ }^{*}$ have angular momentum interpolated linearly from $\Gamma_{\infty}$ at $z=10 \mathrm{~m}$ to $0 \mathrm{~kg}$ $\mathrm{m}^{2} \mathrm{~s}^{-1}$ at $z=0 \mathrm{~m}$ at the lateral boundaries.

\begin{tabular}{|c|cccc|cccc|c|}
\hline Name & $\begin{array}{c}U_{\text {trans }} \\
\left(\mathrm{m} \mathrm{s}^{-1}\right)\end{array}$ & $\begin{array}{c}g \\
\left(\mathrm{~m} \mathrm{~s}^{-2}\right)\end{array}$ & $\sigma / \rho$ & $\begin{array}{c}d \\
\mathrm{~mm}\end{array}$ & $\begin{array}{c}w_{t} \\
\left(\mathrm{~m} \mathrm{~s}^{-1}\right)\end{array}$ & $A_{t}$ & $A_{a}$ & $A_{v}$ & Other \\
\hline dmg3321 & 5 & 9.81 & 2000 & 1 & 5.73 & 0.11 & 2.26 & 8.31 & \\
dmg3322 & 15 & 9.81 & 2000 & 1 & 5.73 & 0.32 & 2.26 & 8.31 & \\
dmg3323 & 25 & 9.81 & 2000 & 1 & 5.73 & 0.53 & 2.26 & 8.31 & \\
dmg2218 & 5 & 2.7667 & 2000 & 2 & 4.58 & 0.11 & 8.00 & 10.4 & \\
dmg2219 & 15 & 2.7667 & 2000 & 2 & 4.58 & 0.32 & 8.00 & 10.4 & \\
dmg2220 & 25 & 2.7667 & 2000 & 2 & 4.58 & 0.53 & 8.00 & 10.4 & \\
\hline
\end{tabular}

Table A.2: A subset of low-swirl runs regridded from run3320-tav.

\begin{tabular}{|c|cccc|cccc|c|}
\hline Name & $\begin{array}{c}U_{\text {trans }} \\
\left(\mathrm{m} \mathrm{s}^{-1}\right)\end{array}$ & $\begin{array}{c}g \\
\left(\mathrm{~m} \mathrm{~s}^{-2}\right)\end{array}$ & $\sigma / \rho$ & $\begin{array}{c}d \\
\mathrm{~mm}\end{array}$ & $\begin{array}{c}w_{t} \\
\left(\mathrm{~m} \mathrm{~s}^{-1}\right)\end{array}$ & $A_{t}$ & $A_{a}$ & $A_{v}$ & Other \\
\hline dmg3242 & 5 & 9.81 & 2000 & 1 & 5.73 & 0.09 & 1.94 & 9.94 & \\
dmg3243 & 15 & 9.81 & 2000 & 1 & 5.73 & 0.26 & 1.94 & 9.94 & \\
dmg3244 & 25 & 9.81 & 2000 & 1 & 5.73 & 0.44 & 1.94 & 9.94 & \\
dmg2226 & 15 & 2.45 & 2000 & 3 & 5.64 & 0.26 & 7.75 & 10.1 & \\
dmg2227 & 25 & 2.45 & 2000 & 3 & 5.64 & 0.44 & 7.75 & 10.1 & \\
\hline
\end{tabular}

Table A.3: A subset of high-swirl runs regridded from run3240-tav. 


\begin{tabular}{|c|c|c|c|c|c|c|c|c|c|}
\hline Name & $\begin{array}{c}U_{\text {trans }} \\
\left(\mathrm{m} \mathrm{s}^{-1}\right)\end{array}$ & $\begin{array}{c}g \\
\left(\mathrm{~m} \mathrm{~s}^{-2}\right)\end{array}$ & $\sigma / \rho$ & $\begin{array}{c}d \\
\mathrm{~mm}\end{array}$ & $\begin{array}{c}w_{t} \\
\left(\mathrm{~m} \mathrm{~s}^{-1}\right)\end{array}$ & $A_{t}$ & $A_{a}$ & $A_{v}$ & Other \\
\hline run2049 & 5 & 9.81 & $\mathrm{n} / \mathrm{a}$ & $\mathrm{n} / \mathrm{a}$ & $\mathrm{n} / \mathrm{a}$ & 0.06 & 7.85 & $\mathrm{n} / \mathrm{a}$ & no debris \\
\hline run2011 & 15 & 9.81 & $\mathrm{n} / \mathrm{a}$ & $\mathrm{n} / \mathrm{a}$ & $\mathrm{n} / \mathrm{a}$ & 0.17 & 7.85 & $\mathrm{n} / \mathrm{a}$ & no debris \\
\hline run2052 & 25 & 9.81 & $\mathrm{n} / \mathrm{a}$ & $\mathrm{n} / \mathrm{a}$ & $\mathrm{n} / \mathrm{a}$ & 0.28 & 7.85 & $\mathrm{n} / \mathrm{a}$ & no debris \\
\hline dmg2033 & 5 & 9.81 & 2000 & 1 & 5.73 & 0.06 & 7.85 & 15.9 & $\min (\Delta x)=8 \mathrm{~m}$ \\
\hline dmg2026 & 15 & 9.81 & 2000 & 1 & 5.73 & 0.17 & 7.85 & 15.9 & $\min (\Delta x)=8 \mathrm{~m}$ \\
\hline dmg2032 & 25 & 9.81 & 2000 & 1 & 5.73 & 0.28 & 7.85 & 15.9 & $\min (\Delta x)=8 \mathrm{~m}$ \\
\hline dmg2049e & 5 & 9.81 & 2000 & 1 & 5.73 & 0.06 & 7.85 & 15.9 & $\min (\Delta x)=2 \mathrm{~m}$ \\
\hline dmg2011e & 15 & 9.81 & 2000 & 1 & 5.73 & 0.17 & 7.85 & 15.9 & $\min (\Delta x)=2 \mathrm{~m}$ \\
\hline dmg2052e & 25 & 9.81 & 2000 & 1 & 5.73 & 0.28 & 7.85 & 15.9 & $\min (\Delta x)=2 \mathrm{~m}$ \\
\hline dmg3031 & 5 & 9.81 & 1000 & 1 & 3.81 & 0.06 & 7.85 & 23.8 & \\
\hline dmg3032 & 15 & 9.81 & 1000 & 1 & 3.81 & 0.17 & 7.85 & 23.8 & \\
\hline dmg3033 & 25 & 9.81 & 1000 & 1 & 3.81 & 0.28 & 7.85 & 23.8 & \\
\hline dmg2245 & 25 & 9.81 & 1500 & 1 & 4.84 & 0.28 & 7.85 & 18.8 & \\
\hline dmg2049 & 5 & 9.81 & 2000 & 1 & 5.73 & 0.06 & 7.85 & 15.9 & \\
\hline dmg2209 & 5 & 9.81 & 2000 & 1 & 5.73 & 0.06 & 7.85 & 15.9 & avail. $=1 \mathrm{~kg} \mathrm{~m}^{-2}$ \\
\hline dmg2011 & 15 & 9.81 & 2000 & 1 & 5.73 & 0.17 & 7.85 & 15.9 & \\
\hline dmg2052 & 25 & 9.81 & 2000 & 1 & 5.73 & 0.28 & 7.85 & 15.9 & \\
\hline dmg2244 & 25 & 9.81 & 3000 & 1 & 7.25 & 0.28 & 7.85 & 12.5 & \\
\hline dmg3024 & 5 & 9.81 & 4000 & 1 & 8.55 & 0.06 & 7.85 & 10.6 & \\
\hline dmg3025 & 15 & 9.81 & 4000 & 1 & 8.55 & 0.17 & 7.85 & 10.6 & \\
\hline dmg3026 & 25 & 9.81 & 4000 & 1 & 8.55 & 0.28 & 7.85 & 10.6 & \\
\hline dmg3021 & 5 & 9.81 & 8000 & 1 & 12.7 & 0.06 & 7.85 & 7.18 & \\
\hline dmg3022 & 15 & 9.81 & 8000 & 1 & 12.7 & 0.17 & 7.85 & 7.18 & \\
\hline dmg3023 & 25 & 9.81 & 8000 & 1 & 12.7 & 0.28 & 7.85 & 7.18 & \\
\hline dmg2274 & 25 & 9.81 & 4000 & 1 & 8.55 & 0.28 & 7.85 & 10.6 & $c_{a}=0.04$ \\
\hline dmg2246 & 25 & 9.81 & 1000 & 1 & 3.81 & 0.28 & 7.85 & 23.8 & $c_{a}=0.36$ \\
\hline dmg2252 & 25 & 9.81 & 1500 & 1 & 4.84 & 0.28 & 7.85 & 18.8 & $c_{a}=0.36$ \\
\hline dmg2070 & 25 & 9.81 & 2000 & 1 & 5.73 & 0.28 & 7.85 & 15.9 & $c_{a}=0.36$ \\
\hline dmg2251 & 25 & 9.81 & 3000 & 1 & 7.25 & 0.28 & 7.85 & 12.5 & $c_{a}=0.36$ \\
\hline dmg2247 & 25 & 9.81 & 4000 & 1 & 8.55 & 0.28 & 7.85 & 10.6 & $c_{a}=0.36$ \\
\hline dmg2248 & 25 & 9.81 & 8000 & 1 & 12.7 & 0.28 & 7.85 & 7.18 & $c_{a}=0.36$ \\
\hline dmg3304 & 5 & 9.81 & 2000 & 1 & 5.73 & 0.06 & 7.85 & 15.9 & $c_{d}=0.30, c_{t}=2.0$ \\
\hline dmg3305 & 15 & 9.81 & 2000 & 1 & 5.73 & 0.17 & 7.85 & 15.9 & $c_{d}=0.30, c_{t}=2.0$ \\
\hline dmg3306 & 25 & 9.81 & 2000 & 1 & 5.73 & 0.28 & 7.85 & 15.9 & $c_{d}=0.30, c_{t}=2.0$ \\
\hline dmg2283 & 25 & 9.81 & 4000 & 1 & 8.55 & 0.28 & 7.85 & 10.6 & $c_{b}=0.25$ \\
\hline dmg2285 & 25 & 9.81 & 4000 & 1 & 8.55 & 0.28 & 7.85 & 10.6 & $c_{u}=0.20$ \\
\hline dmg2180 & 25 & 9.81 & 500 & 8 & 10.3 & 0.28 & 7.85 & 8.82 & $\sigma d$ test \\
\hline dmg2185 & 25 & 9.81 & 100 & 40 & 10.9 & 0.28 & 7.85 & 8.34 & $\sigma d$ test \\
\hline dmg2288 & 25 & 9.81 & 20000 & 0.2 & 6.09 & 0.28 & 7.85 & 14.9 & $\sigma d$ test \\
\hline dmg2192 & 5 & 39.24 & 2000 & 0.6 & 8.77 & 0.06 & 1.96 & 10.4 & \\
\hline dmg2193 & 15 & 39.24 & 2000 & 0.6 & 8.77 & 0.17 & 1.96 & 10.4 & \\
\hline dmg2203 & 30 & 39.24 & 2000 & 0.6 & 8.77 & 0.33 & 1.96 & 10.4 & \\
\hline dmg2279 & 15 & 150 & 275 & 1 & 8.80 & 0.17 & 0.51 & 10.3 & \\
\hline
\end{tabular}

Table A.4: A subset of medium-swirl runs regridded from run3300-tav. 


\begin{tabular}{|c|c|c|c|c|c|c|c|c|c|}
\hline Name & $\begin{array}{c}U_{\text {trans }} \\
\left(\mathrm{m} \mathrm{s}^{-1}\right)\end{array}$ & $\begin{array}{c}g \\
\left(\mathrm{~m} \mathrm{~s}^{-2}\right)\end{array}$ & $\sigma / \rho$ & $\begin{array}{c}d \\
\mathrm{~mm}\end{array}$ & $\begin{array}{c}w_{t} \\
\left(\mathrm{~m} \mathrm{~s}^{-1}\right)\end{array}$ & $A_{t}$ & $A_{a}$ & $A_{v}$ & Other \\
\hline run2059 & 5 & 9.81 & $\mathrm{n} / \mathrm{a}$ & $\mathrm{n} / \mathrm{a}$ & $\mathrm{n} / \mathrm{a}$ & 0.07 & 2.39 & $\mathrm{n} / \mathrm{a}$ & no debris \\
\hline run2014 & 15 & 9.81 & $\mathrm{n} / \mathrm{a}$ & $\mathrm{n} / \mathrm{a}$ & $\mathrm{n} / \mathrm{a}$ & 0.21 & 2.39 & $\mathrm{n} / \mathrm{a}$ & no debris \\
\hline run2060 & 25 & 9.81 & $\mathrm{n} / \mathrm{a}$ & $\mathrm{n} / \mathrm{a}$ & $\mathrm{n} / \mathrm{a}$ & 0.36 & 2.39 & $\mathrm{n} / \mathrm{a}$ & no debris \\
\hline dmg2035 & 5 & 9.81 & 2000 & 1 & 5.73 & 0.07 & 2.39 & 12.2 & $\min (\Delta x)=8 \mathrm{~m}$ \\
\hline dmg2028 & 15 & 9.81 & 2000 & 1 & 5.73 & 0.21 & 2.39 & 12.2 & $\min (\Delta x)=8 \mathrm{~m}$ \\
\hline dmg2034 & 25 & 9.81 & 2000 & 1 & 5.73 & 0.36 & 2.39 & 12.2 & $\min (\Delta x)=8 \mathrm{~m}$ \\
\hline dmg2059e & 5 & 9.81 & 2000 & 1 & 5.73 & 0.07 & 2.39 & 12.2 & $\min (\Delta x)=2 \mathrm{~m}$ \\
\hline dmg2014e & 15 & 9.81 & 2000 & 1 & 5.73 & 0.21 & 2.39 & 12.2 & $\min (\Delta x)=2 \mathrm{~m}$ \\
\hline dmg2060e & 25 & 9.81 & 2000 & 1 & 5.73 & 0.36 & 2.39 & 12.2 & $\min (\Delta x)=2 \mathrm{~m}$ \\
\hline dmg2058 & 0 & 9.81 & 2000 & 1 & 5.73 & 0 & 2.39 & 12.2 & stationary \\
\hline dmg2177 & 5 & 9.81 & 1000 & 1 & 3.81 & 0.07 & 2.39 & 18.4 & \\
\hline dmg2178 & 15 & 9.81 & 1000 & 1 & 3.81 & 0.21 & 2.39 & 18.4 & \\
\hline dmg2179 & 25 & 9.81 & 1000 & 1 & 3.81 & 0.36 & 2.39 & 18.4 & \\
\hline dmg2059 & 5 & 9.81 & 2000 & 1 & 5.73 & 0.07 & 2.39 & 12.2 & \\
\hline dmg2165 & 5 & 9.81 & 2000 & 1 & 5.73 & 0.07 & 2.39 & 12.2 & avail. $=1 \mathrm{~kg} \mathrm{~m}^{-2}$ \\
\hline dmg2014 & 15 & 9.81 & 2000 & 1 & 5.73 & 0.21 & 2.39 & 12.2 & \\
\hline dmg2060 & 25 & 9.81 & 2000 & 1 & 5.73 & 0.36 & 2.39 & 12.2 & \\
\hline dmg2080 & 5 & 9.81 & 4000 & 1 & 8.55 & 0.07 & 2.39 & 8.18 & \\
\hline dmg2081 & 15 & 9.81 & 4000 & 1 & 8.55 & 0.21 & 2.39 & 8.18 & \\
\hline dmg2082 & 25 & 9.81 & 4000 & 1 & 8.55 & 0.36 & 2.39 & 8.18 & \\
\hline dmg2084 & 35 & 9.81 & 4000 & 1 & 8.55 & 0.36 & 2.39 & 8.18 & \\
\hline dmg2090 & 5 & 9.81 & 8000 & 1 & 12.65 & 0.07 & 2.39 & 5.53 & \\
\hline dmg2091 & 15 & 9.81 & 8000 & 1 & 12.65 & 0.21 & 2.39 & 5.53 & \\
\hline dmg2092 & 25 & 9.81 & 8000 & 1 & 12.65 & 0.36 & 2.39 & 5.53 & \\
\hline dmg2277 & 25 & 9.81 & 4000 & 1 & 8.55 & 0.36 & 2.39 & 8.18 & $c_{a}=0.04$ \\
\hline dmg2276 & 25 & 9.81 & 4000 & 1 & 8.55 & 0.36 & 2.39 & 8.18 & $c_{a}=0.36$ \\
\hline dmg2045 & 5 & 9.81 & 2000 & 1 & 5.73 & 0.07 & 2.39 & 12.2 & $c_{d}=0.30, c_{t}=2.0$ \\
\hline dmg2047 & 15 & 9.81 & 2000 & 1 & 5.73 & 0.21 & 2.39 & 12.2 & $c_{d}=0.30, c_{t}=2.0$ \\
\hline dmg2042 & 25 & 9.81 & 2000 & 1 & 5.73 & 0.36 & 2.39 & 12.2 & $c_{d}=0.30, c_{t}=2.0$ \\
\hline dmg2282 & 25 & 9.81 & 4000 & 1 & 8.55 & 0.36 & 2.39 & 8.18 & $c_{b}=0.25$ \\
\hline dmg2284 & 25 & 9.81 & 4000 & 1 & 8.55 & 0.50 & 2.39 & 8.18 & $c_{u}=0.20$ \\
\hline dmg2181 & 15 & 9.81 & 250 & 8 & 7.14 & 0.21 & 2.39 & 9.80 & \\
\hline sig1115 & 15 & 9.81 & 100 & 20 & 7.47 & 0.21 & 2.39 & 9.37 & \\
\hline sig2015 & 15 & 9.81 & 11330 & 0.177 & 3.69 & 0.21 & 2.39 & 19.0 & \\
\hline dmg2212 & 5 & 2.9727 & 6500 & 1 & 5.68 & 0.07 & 7.89 & 12.3 & \\
\hline dmg2195 & 25 & 2.9727 & 6500 & 1 & 5.68 & 0.36 & 7.89 & 12.3 & \\
\hline dmg2280 & 15 & 46.9 & 570 & 1 & 6.86 & 0.21 & 0.50 & 10.2 & \\
\hline
\end{tabular}

Table A.5: A subset of high-swirl runs regridded from run2057-tav. 


\begin{tabular}{|c|cccc|cccc|l|}
\hline Name & $\begin{array}{c}U_{\text {trans }} \\
\left(\mathrm{m} \mathrm{s}^{-1}\right)\end{array}$ & $\begin{array}{c}g \\
\left(\mathrm{~m} \mathrm{~s}^{-2}\right)\end{array}$ & $\sigma / \rho$ & $\begin{array}{c}d \\
\mathrm{~mm}\end{array}$ & $\begin{array}{c}w_{t} \\
\left(\mathrm{~m} \mathrm{~s}^{-1}\right)\end{array}$ & $A_{t}$ & $A_{a}$ & $A_{v}$ & Other \\
\hline dmg2249 & 25 & 9.81 & 1000 & 1 & 3.81 & 0.28 & 7.85 & 23.8 & $c_{a}=0.04$ \\
& & & 4000 & 1 & 8.55 & & & 10.6 & $c_{a}=0.04$ \\
& & & 8000 & 1 & 12.65 & & & 7.18 & $c_{a}=0.04$ \\
3sp3026 & 25 & 9.81 & 1000 & 1 & 3.81 & 0.28 & 7.85 & 23.8 & $c_{a}=0.12$ \\
& & & 4000 & 1 & 8.55 & & & 10.6 & $c_{a}=0.12$ \\
& & & 8000 & 1 & 12.65 & & & 7.18 & $c_{a}=0.12$ \\
dmg2267 & 25 & 9.81 & 1000 & 1 & 3.81 & 0.28 & 7.85 & 23.8 & $c_{a}=0.0950$ \\
& & & 4000 & 1 & 8.55 & & & 10.6 & $c_{a}=0.0175$ \\
& & & 8000 & 1 & 12.65 & & & 7.18 & $c_{a}=0.0070$ \\
\hline
\end{tabular}

Table A.6: A subset of multispecies, medium-swirl runs regridded from run3300-tav. 


\section{Bibliography}

E M Agee, J T Snow, and P R Clare. Multiple vortex features in the tornado cyclone and the occurrence of tornado families. Monthly Weather Review, (104):552, 1975.

E M Agee, J T Snow, F S Nickerson, P R Clare, C R Church, and L A Schaal. An observational study of the West Lafayette, Indiana, tornado of 20 March 1976. Monthly Weather Review, (105):893, 1977.

C R Alexander and J M Wurman. Updated mobile radar climatology of supercell tornado structures and dynamics. Proceedings of the 24th AMS Conference on Severe Local Storms, page 19.4, 2008.

J Bech, M Gayb, M Arana, F Figuerolaa, J Amaroa, and J Arsc. Tornado damage analysis of a forest area using site survey observations, radar data and a simple analytical vortex model. Atmospheric Research, (93):118, 2009.

V Beck. Determination of tornado intensity from forest damage. Proceedings of 24 th conference on severe local storms, page 8B.2, 2008.

V Beck and N Dotzek. Reconstruction of near-surface tornado wind fields from forest damage. J. Appl. Meteor. Climatol., (49):1517, 2010.

H B Bluestein and J H Golden. A review of tornado observations. AGU Geophysical Monograph 79, ed. C Church, D Burgess, C Doswell, R Davies-Jones, page 319, 1993. 
H B Bluestein and W P Unruh. Observations of the wind field in tornadoes, funnel clouds, and wall clouds with a portable Doppler radar. Bulletin of the American Meteorological Society, (70):1514, 1989.

R P Davies-Jones, D W Burgess, L R Lemon, and D Purcell. Interpretation of surface marks and debris patterns from the 24 May 1973 Union City, Oklahoma tornado. Monthly Weather Review, (106):12, 1978.

C A Doswell, H E Brooks, and N Dotzek. On the implementation of the enhanced Fujita scale in the USA. Atmospheric Research, (93):554, 2009.

D C Dowell, C R Alexander, J M Wurman, and L J Wicker. Centrifuging of hydrometeors and debris in tornadoes: Radar-reflectivity patterns and wind-measurement errors. Journal of the Atmospheric Sciences, page 1501, 2005.

G S Forbes and R M Wakimoto. A concentrated outbreak of tornadoes, downbursts and microbursts, and implications regarding vortex classification. Monthly Weather Review, (111):220, 1982 .

T T Fujita. Aerial survey of the Palm Sunday tornadoes of April 11, 1965. Technical Report SMRP Res. Pap. 49, The University of Chicago, January 1966.

T T Fujita. Estimated wind speeds of the Palm Sunday tornadoes. Technical Report SMRP Res. Pap. 53, The University of Chicago, April 1967.

T T Fujita. Tornadoes and downbursts in the context of generalized planetary scales. Journal of the Atmospheric Sciences, (38):1511, 1981.

T T Fujita, D L Bradbury, and P G Black. Estimation of tornado wind speed from characteristic ground marks. Technical Report SMRP Res. Pap. 69, The University of Chicago, October 1967. 
T T Fujita, D L Bradbury, and C F Thullenar. Palm Sunday tornadoes of April 11, 1965. Monthly Weather Review, (98):29, 1970.

T T Fujita, G S Forbes, and T A Umenhofer. Close-up view of 20 March 1976 tornadoes: Sinking cloud tops to suction vortices. Weatherwise, (29):116, 1976a.

T T Fujita, G S Forbes, T A Umenhofer, E W Pearl, and J J Tecson. Photogrammetric analyses of tornadoes. Symp. on Tornadoes: Assessment of Knowledge and Implications for Man, Texas Tech. University, Lubbock, TX, page 107, 1976b.

B Gong. Large eddy simulation of the effects of debris on tornado dynamics. Doctoral Thesis, Department of Mechanical Engineering, West Virginia University, Morgantown, WV, 2006.

A P Holland, A J Riordan, and E C Franklin. A simple model for simulating tornado damage in forests. J. Appl. Meteor. Climatol., (45):1597, 2006.

J P Letzmann. Das bewegungsfeld im fu einer fortschreitenden wind- oder wasserhose (the flow field at the base of an advancing tornado). Ph.D. thesis, University Helsingfors, Acta et Commentationes Universitatis Dorpatensis, 1923a.

J P Letzmann. Fortschreitende luftwirbel (advancing air vortices). Meteor. Z., (42):41, $1923 b$.

D C Lewellen. Tornado corner flow dynamics; presentation given at Iowa State University. 2007.

D C Lewellen and W S Lewellen. Near-surface intensification of tornado vortices. Journal of the Atmospheric Sciences, 64:2176, 2007a.

D C Lewellen and W S Lewellen. Near-surface vortex intensification through corner flow collapse. Journal of the Atmospheric Sciences, 64:2195, 2007b. 
D C Lewellen and M I Zimmerman. Using simulated tornado surface marks to help decipher near-ground wind fields. Proceedings of the 24th AMS Conference on Severe Local Storms, page $13.5,2008$.

D C Lewellen, W S Lewellen, and J Xia. The influence of a local swirl ratio on tornado intensification near the surface. Journal of the Atmospheric Sciences, 57:527, 2000.

D C Lewellen, B Gong, and W S Lewellen. Effects of debris on near-surface tornado dynamics. Proceedings of the 22nd AMS Conference on Severe Local Storms, page 15.5, 2004.

D C Lewellen, B Gong, and W S Lewellen. Effects of finescale debris on near-surface tornado dynamics. Journal of the Atmospheric Sciences, 65:3247, 2008.

W S Lewellen. Tornado vortex theory. the tornado: Its structure, dynamics, prediction, and hazards. Geophys. Monogr., No. 79, (79):19, 1973.

W S Lewellen. Theoretical models of the tornado vortex. Symp. on Tornadoes: Assessment of Knowledge and Implications for Man, Texas Tech. University, Lubbock, TX, page 107, 1976.

W S Lewellen, D C Lewellen, and R I Sykes. Large eddy simulation of a tornado's interaction with the surface. Journal of the Atmospheric Sciences, 54:581, 1997.

$\mathrm{T}$ Lindeberg. Edge detection and ridge detection with automatic scale selection. IEEE Computer Society Conference on Computer Vision and Pattern Recognition, Proceedings, page 465, 1996.

F E Marble. Dynamics of dusty gases. Ann. Rev. Fluid Mech., (2):397, 1970.

J McDonald and K C Mehta. A recommendation for an Enhanced Fujita Scale (EF-scale), October 2006.

N E Prosser. Aerial photographs of a tornado path in Nebraska. May 5, 1964. Monthly Weather Review, (92):593, 1964. 
USDA NRCS Soil Survey Staff. Soil Taxonomy: A Basic System of Soil Classification for Making and Interpreting Soil Surveys, 2nd ed. United States Department of Agriculture, Natural Resources Conservation Service, 1999.

A Whipple. Storm. Time Life Books, Alexandria, Virginia, 1982.

F M White. Viscous Fluid Flow. McGraw-Hill, 1991.

J Wurman, L Wicker, Y Richardson, P Markowski, D Dowell, D Burgess, and H Bluestein. VORTEX2: The verification of the origins of rotation in tornadoes experiment. Proceedings of the 25th Conference on Severe Local Storms, page 5.1, 2010.

J M Wurman, J Straka, E Rasmussen, M Randall, and A Zahari. Design and deployment of a portable, pencil-beam, pulsed, 3-cm Doppler radar. Journal of Atmospheric and Oceanic Technology, (14):1502, 1997.

J Xia, W S Lewellen, and D C Lewellen. Influence of mach number on tornado corner flow dynamics. Journal of the Atmospheric Sciences, 60:2820, 2003. 